\title{
GOETHE
}

UNIVERSITÄT

FRANKFURT AM MAIN

\section{TRANSPORT PROPERTIES \\ OF THE STRONGLY-INTERACTING \\ QUARK-GLUON PLASMA}

\author{
Dissertation \\ zur Erlangung des Doktorgrades \\ der Naturwissenschaften \\ vorgelegt beim Fachbereich Physik \\ der Johann Wolfgang Goethe-Universität \\ in Frankfurt am Main \\ von \\ Olga Soloveva \\ aus Uljanowsk, Russland
}

Frankfurt 2021

(D 30) 
vom Fachbereich Physik (13) der

Johann Wolfgang Goethe-Universität als Dissertation angenommen.

Dekan: Prof. Dr. Harald Appelshäuser

Gutachter: Prof. Dr. Elena Bratkovskaya und Prof. Dr. Dr. h.c. Marcus Bleicher Datum der Disputation: 22.12.2021 


\section{Zusammenfassung}

"Dass ich nicht mehr mit sauerm Schweiß

Zu sagen brauche, was ich nicht weiss;

Dass ich erkenne, was die Welt

Im Innersten zusammenhaelt,

Schau alle Wirkenskraft und Samen,

Und tu nicht mehr in Worten kramen."

Faust, Johann Wolfgang von Goethe

Das Verständnis der Phasenübergänge in stark wechselwirkender Kernmaterie und der Eigenschaften des Quark-Gluon-Plasmas (QGP), das bei relativistischen Schwerionenkollisionen erzeugt wird, ist gegenwärtig eine der größten Herausforderungen der theoretischen Physik.

Bei ultra-relativistischen Energien am Large Hadron Collider (LHC) oder am Relativistic Heavy-Ion Collider (RHIC) hat das QGP, das im zentralen Wechselwirkungsvolumen entsteht, eine Nettobaryonendichte oder ein chemisches Baryonenpotenzial von nahezu Null $\left(\mu_{B} \approx 0\right)$, und der Übergang von einem freien Zustand der Quarks und Gluonen zum eingeschlossenen (confined) Zustand der Hadronen ist ein schneller, aber kontinuierlicher Übergang. Das derzeitige Interesse gilt jedoch Kollisionen bei niedrigeren Laborenergien, bei denen die Nettobaryonendichte höher ist und das chemische Potenzial der Baryonen entsprechend auch höher ist. Einige theoretische Modelle sagen dort einen möglichen Phasenübergang erster Ordnung sowie einen kritischen Endpunkt (CEP) voraus. Der genaue Ort des CEP im Phasendiagramm ist jedoch noch unbekannt.

Um den Bereich des QCD-Phasendiagramms bei endlichen baryonchemischen Potenzialen zu erreichen, wird in laufenden Experimenten die Labornergie gesenkt und Observablen bei zentraler Rapidität gemessen. Bisher haben die Programme Beam Energy Scan (BES) und Fixed-Target (FXT) am RHIC eine Reihe von $\mathrm{Au}+\mathrm{Au}-$ Kollisionen im Bereich von $\sqrt{s_{N N}}$ von $200 \mathrm{GeV}$ bis hinunter zu $3 \mathrm{GeV}$ durchgeführt. Nach den statistischen Modellen variiert das entsprechende baryonische chemische Potential - beim chemischen Einfrieren - von $\mu_{B} \approx 20 \mathrm{MeV}$ bei $\sqrt{s_{N N}}=200 \mathrm{GeV}$ bis $\approx 420 \mathrm{MeV}$ bei $\sqrt{s_{N N}}=7.7 \mathrm{GeV}$ und $\mu_{B} \approx 750 \mathrm{MeV}$ bei $\sqrt{s_{N N}}=3 \mathrm{GeV}$ für FXT. Um das Vorhandensein eines CEP und mögliche Auswirkungen eines Phasenübergangs erster Ordnung zu untersuchen, kann ein Anstieg des chemischen Baryonenpotentials durch weitere Absenkung der Kollisionsenergie in den zukünftigen Experimenten an FAIR (Facility for Antiproton and Ion Research) und NICA (Nuclotron-based Ion Collider fAcility) erreicht werden, die insbesondere das QGPPhasendiagramm bei moderaten und höheren $\mu_{B}$-Werten untersuchen. Dies ist der am wenigsten erforschte Bereich des QCD-Phasendiagramms, aber von besonderem Interesse für zukünftige experimentelle Programme und theoretische Studien. 
Um diese Untersuchungen in einem viskosen hydrodynamischen Modell durchführen zu können, muss man die Zustandsgleichung (EoS) der stark wechselwirkenden Materie und auch die Transportkoeffizienten kennen. Die zeitliche Entwicklung des QGP-Mediums, das in Schwerionen-Kollisionen (HICs) erzeugt wird, kann auch in mikroskopischen Transportansätzen untersucht werden, die die zeitliche Entwicklung der Freiheitsgrade des Systems liefern. Sie erfordern zusätzlich die Kenntnis der mikroskopischen Eigenschaften der partonischen Freiheitsgrade, wie effektive Massen, Breiten und Wirkungsquerschnitte, die alle von $\mu_{B}$ und $T$ abhängen können. Der große Wert der laufenden Kopplung erfordert nichtperturbative Methoden wie 1QCD-Rechnungen oder effektive Modelle mit einem phüomenologischen Input. Daher ist es bekanntermaßen schwierig, die thermodynamischen Eigenschaften der QCD-Materie im nicht eingeschlossenen Zustand abzuschätzen, insbesondere in der Nähe eines Phasenübergangs. Während lQCD-Berechnungen bei verschwindendem baryonischem Potential gut etabliert sind, ist die Ausweitung dieser Rechnungen auf ein großes baryonisches Potential aufgrund des Fermionen-Vorzeichen-Problems nicht etabliert. Eine Möglichkeit, thermodynamische Funktionen bei $\mu_{B}>0$ zu untersuchen, ist die Verwendung einer Taylor-Entwicklung der Verteilungsfunktion in der Nähe von $\mu_{B}=$ 0. Sie teilt mit anderen Ansätzen, die für $\mu_{B}=0$ entwickelt wurden, dass die Unsicherheit der Vorhersagen mit der Zunahme von $\mu_{B}$ zunimmt. Um die Untersuchung der Transportkoeffizienten auf den Teil des Phasendiagramms auszudehnen, in dem der Phasenübergang möglicherweise von einem Crossover zu einem erster Ordnung wechselt, ist es notwendig, auf effektive Modelle zurückzugreifen.

Ziel dieser Dissertation ist es, die Gleichgewichts- und Nichtgleichgewichts-Eigenschaften des stark wechselwirkenden QGP-Mediums nahe dem Phasenübergang unter extremen Bedingungen von hohen $T$ und hohen Baryonendichten mit Hilfe der kinetischen Theorie im Rahmen von effektiven Modellen zu untersuchen. Wir werden zunächst die thermodynamischen und Transporteigenschaften des QGPs in der Nähe des Gleichgewichts auf der Basis des DQPM im Bereich moderater chemischer Baryonenpotentiale $\mu_{B} \geq 0.5 \mathrm{GeV}$ untersuchen. Insbesondere werden die EoS und die Schallgeschwindigkeit sowie die Transportkoeffizienten des QGP auf der Grundlage des DQPM bei endlichen $T$ und $\mu_{B}$ berechnet. Transportkoeffizienten sind besonders interessant, da sie Informationen über die Wechselwirkungen im Medium erlauben, das im Gleichgewicht durch eine Temperatur $T$ und ein chemisches Potential $\mu_{B}$ charakterisiert werden kann. Unter Berücksichtigung der Transportkoeffizienten und der EoS der QGP-Phase vergleichen wir unsere Ergebnisse mit verschiedenen Resultaten aus der Literatur, in denen Transportkoeffizienten des QGPs auf Basis von effektiven Modellen vorwiegend bei Null oder kleinem chemischen Potentialen untersucht wurden.

Darüber hinaus werden in Kapitel 3 die Gleichgewichtseigenschaften des QGPs und insbesondere die Auswirkungen der $\mu_{B}$-Abhängigkeit der thermodynamischen und Transporteigenschaften des QGPs im Rahmen des erweiterten PHSD-Transportansatzes untersucht, der die vollständige Entwicklung des Systems einschließlich der partonischen Phase umfasst. Die Entwicklung des PHSD-Transportansatzes wird in der partonischen Phase erweitert, indem explizit die gesamt- und differentiellen partonischen Streuquerschnitte auf der 
Grundlage des DQPM berechnet und bei der tatsächlichen Temperatur $T$ und dem baryonischen chemischen Potential $\mu_{B}$ in jeder einzelnen Raum-Zeit-Zelle, in der die partonische Streuung stattfindet, ausgewertet werden.

Um die Spuren der $\mu_{B}$-Abhn̈gigkeit des QGPs in den Observablen zu untersuchen, werden die Ergebnisse von PHSD5.0 (mit $\mu_{B}$-Abhängigkeiten) mit den Ergebnissen von PHSD5.0 für $\mu_{B}=0$ sowie mit PHSD4.0, in dem die Massen/Breiten der Quarks und Gluonen sowie deren Wechselwirkungsquerschnitte nur von $T$ abhängen, verglichen. Wir diskutieren die PHSD-Ergebnisse für verschiedene Observablen: (i) Rapiditäts- und $p_{T^{-}}$Verteilungen von identifizierten Hadronen für symmetrische $\mathrm{Au}+\mathrm{Au}-$ und $\mathrm{Pb}+\mathrm{Pb}$ - Kollisionen bei Energien von $30 \mathrm{AGeV}$ (zukünftige NICA-Energie) sowie für die RHIC-Spitzenenergie von $\sqrt{s_{N N}}=200 \mathrm{GeV}$; (ii) gerichteter Fluss $v_{1}$ von identifizierten Hadronen für $A u+A u$ bei invarianter Energie $\sqrt{s_{N N}}=27 \mathrm{GeV}$ und $200 \mathrm{GeV}$; (iii) elliptischer Fluss $v_{2}$ der identifizierten Hadronen für $\mathrm{Au}+\mathrm{Au}$ bei invarianten Energien $\sqrt{s_{N N}}=27$ und $200 \mathrm{GeV}$.

Der Vergleich der "BulkObservablen für $\mathrm{Au}+\mathrm{Au}-$ Kollisionen innerhalb der drei PHSDEinstellungen hat gezeigt, dass sie eine recht geringe Empfindlichkeit gegenüber den $\mu_{B^{-}}$ Abhängigkeiten der Partoneigenschaften (Massen und Breiten) und ihrer Wechselwirkungsquerschnitte aufweisen, sodass die Ergebnisse von PHSD5.0 mit und ohne $\mu_{B}$ sehr nahe beieinander liegen. Nur im Fall von Kaonen, Antiprotonen $\bar{p}$ und Antihyperonen $\bar{\Lambda}+\bar{\Sigma}^{0}$ konnte ein kleiner Unterschied zwischen PHSD4.0 und PHSD5.0 bei den höchsten SPSund RHIC-Energien festgestellt werden.

Wir finden nur geringe Unterschiede zwischen den Ergebnissen von PHSD4.0 und PHSD5.0 für die hier betrachteten hadronischen Observablen sowohl bei hohen als auch bei mittleren Energien. Dies hängt damit zusammen, dass bei hohen Energien, wo die Materie vom QGP dominiert wird, ein sehr kleines chemisches Baryonenpotential $\mu_{B}$ in zentralen Kollisionen bei mittlerer Rapidität gemessen wird, während mit abnehmender Energie und größerem $\mu_{B}$ der Anteil des QGPs rapide abnimmt, sodass die endgültigen Beobachtungswerte insgesamt von den Hadronen dominiert werden, die an der hadronischen Rückstreuung teilgenommen haben, und somit die Information über ihren QGP-Ursprung verwaschen oder verloren geht.

In Kapitel 4 betrachten wir die Transportkoeffizienten von QGP-Materie im erweiterten Polyakov-NJL-Modell entlang der Übergangslinie für moderate Werte des chemischen Baryonenpotenzials $0 \leq \mu_{B} \leq 0.9 \mathrm{GeV}$ sowie in der Nähe des kritischen Endpunkts (CEP) und bei großem chemischen Baryonenpotenzial $\mu_{B}=1.2 \mathrm{GeV}$, wo ein Phasenübergang erster Ordnung stattfindet. Wir untersuchen, wie die Natur der Freiheitsgrade die Transporteigenschaften des QGPs beeinflusst. Darüber hinaus demonstrieren wir die Auswirkungen des Phasenübergangs erster Ordnung und des CEP auf die Transportkoeffizienten im dekonfinierten QCD-Medium.

Darüber hinaus wird in Kapitel 5 eine phänomenologische Erweiterung des DQPM auf große baryonchemische Potentiale $\mu_{B}$ einschließlich der Region mit einem möglichen CEP und späterem Phasenübergang erster Ordnung betrachtet. Eines der wichtigsten Merkmale des Modells ist das Auftreten einer 'kritischen' Skalierung in der Nähe des CEP. Das Hauptziel des vorgestellten Modells besteht darin, die mikroskopischen und makroskopi- 
schen Eigenschaften der partonischen Freiheitsgrade für den Bereich des Phasendiagramms bereitzustellen, der durch moderates $T$ und moderates oder hohes $\mu_{B}$ gekennzeichnet ist. Ihre Kenntnis ermöglicht es anschließend, die Transportkoeffizienten sowie die EoS, die Bestandteile viskoser hydrodynamischer Simulationen sind, zu berechnen. Die EoS, die Schallgeschwindigkeit und die spezifische Wärme sowie die Transportkoeffizienten für $T>T_{c}$ werden für einen weiten Bereich von $\mu_{B}$ vorgestellt, wobei zwei Einstellungen der chemischen Potentiale der seltsamen (strange) Quarks (I) $\mu_{q}=\mu_{u}=\mu_{s}=\mu_{B} / 3$ und (II) $\mu_{s}=0, \mu_{u}=\mu_{d}=\mu_{B} / 3$ verwendet werden, die für hydrodynamische Simulationen von Interesse sind. Darüber hinaus diskutieren wir den Vergleich mit den Transportkoeffizienten des PNJL-Ansatzes, der ein sehr ähnliches Phasendiagramm, aber andere Freiheitsgrade hat, d.h. wechselwirkende masselose Quarks und keine Gluonen. Der Vergleich der Transportkoeffizienten zeigt, dass sie in der Tat von den Eigenschaften der Freiheitsgrade abhängen und in zwei Theorien mit fast dem gleichen Phasendiagramm recht unterschiedlich sein können.

Diese Dissertation basiert auf folgenden Publikationen:

1. Exploring the partonic phase at finite chemical potential within an extended off-shell transport approach.

P. Moreau, O. Soloveva, L. Oliva, T. Song, W. Cassing \& E. Bratkovskaya, Phys. Rev. C 100 (2019) no.1, 014911 [1].

2. Transport coefficients for the hot quark-gluon plasma at finite chemical potential $\mu_{B}$. O. Soloveva, P. Moreau \& E. Bratkovskaya, Phys. Rev. C 101 (2020) no.4, 045203 [2].

3. Transport coefficients of hot and dense matter.

O. Soloveva, P. Moreau, L. Oliva, T. Song, W. Cassing \& E. Bratkovskaya, Springer Proc. Phys. 250 (2020), 447-451 [3].

4. Exploring the partonic phase at finite chemical potential in and out-of equilibrium.

O. Soloveva, P. Moreau, L. Oliva, V. Voronyuk, V. Kireyeu, T. Song

\& E. Bratkovskaya,

Particles 3 (2020) no.1, 178-192 [4].

5. Shear viscosity and electric conductivity of a hot and dense QGP with a chiral phase transition.

O. Soloveva, D. Fuseau, J. Aichelin \& E. Bratkovskaya,

Phys. Rev. C 103 (2021) no.5, 054901 [5].

6. Diffusion coefficient matrix of the strongly interacting quark-gluon plasma.

J. A. Fotakis, O. Soloveva, C. Greiner, O. Kaczmarek \& E. Bratkovskaya,

Phys. Rev. D 104 (2021) no.3, 034014 [6]. 
7. Properties of the QGP created in heavy-ion collisions.

P. Moreau, O. Soloveva, I. Grishmanovskii, V. Voronyuk, L. Oliva, T. Song, V. Kireyeu, G. Coci \& E. Bratkovskaya

Astron. Nachr., 342, 715 (2021) [7].

8. QCD at finite chemical potential in and out-of equilibrium.

O. Soloveva, P. Moreau \& E. Bratkovskaya,

Phys. Scripta 96 (2021) no.12, 124067 [8].

9. Transport properties and equation-of-state of hot and dense QGP matter near the critical end-point in the phenomenological dynamical quasi-particle model.

O. Soloveva, J. Aichelin \& E. Bratkovskaya, arXiv:2108.08561 [hep-ph] (2021) [9]. 


\section{Contents}

Zusammenfassung I I

$\begin{array}{ll}\text { Introduction } & 1\end{array}$

$1 \quad$ Theory of Strong Interactions 3

1.1 Fundamental properties of QCD . . . . . . . . . . . . . . . . 3

1.2 Equilibrium properties of QCD matter: Lattice QCD . . . . . . . . . . 6

1.3 Symmetries and phases of QCD . . . . . . . . . . . . . . . 9

1.4 Phase diagram of QCD matter . . . . . . . . . . . . . . . . . 12

1.5 Dynamical properties of QCD matter:

Modelling the heavy-ion collisions . . . . . . . . . . . . . . . . 13

2 Dynamical quasiparticle model of QGP at finite $\mu_{B} \quad 16$

2.1 Basics of the off-shell quasiparticle model . . . . . . . . . . . . . . . 17

2.2 Thermodynamic quantities in the DQPM for the crossover region . . . . . . 21

2.3 Partonic interactions: cross sections and interaction rates . . . . . . . . 24

2.4 Transport coefficients of the QGP at finite $\mathrm{T}$ and $\mu_{B} \ldots \ldots . \ldots 30$

2.4.1 Parton relaxation times . . . . . . . . . . . . . . 30

2.4 .2 Shear and bulk viscosities . . . . . . . . . . . . . . . . . . 32

2.4 .3 Electric conductivity . . . . . . . . . . . . . . . . 36

2.4 .4 Baryon diffusion . . . . . . . . . . . . . . . . . . . . . 38

2.4.5 Diffusion coefficient matrix: calculations within

the RTA and the Chapman-Enskog approximation . . . . . . . . 40

2.5 Summary and Conclusions to Section 2 . . . . . . . . . . . . . . . . 49

3 Dynamical evolution of QCD matter from transport simulations $\quad 50$

3.1 Generalized transport equations from the Kadanoff-Baym equations . . . . 51

3.2 Generalized test-particle representation . . . . . . . . . . . . . . . . 54

3.3 Parton-Hadron-String Dynamics transport approach in a nutshell . . . . . 56

3.4 Modelling Heavy-Ion Collisions . . . . . . . . . . . . . . . . . . . . . . 60

3.4.1 Bulk observables from relativistic nucleus-nucleus collisions . . . . 63

3.4 .2 Asimuthal anisotropy in the HICs . . . . . . . . . . . . . . . . 68

3.4 .3 Directed Flow $v_{1} \ldots \ldots \ldots \ldots \ldots$

3.4 .4 Elliptic Flow $v_{2} \ldots \ldots \ldots \ldots \ldots 71$

3.5 Conclusions to Section $3 \ldots \ldots \ldots$. . . . . . . . . . . . . 75

4 Critical end point and 1st order phase transition of QCD matter within $\begin{array}{ll}\text { the PNJL model } & \mathbf{7 7}\end{array}$

4.1 Basic ideas of the Nambu-Jona Lasinio model . . . . . . . . . . . . . . . 78

4.2 Polyakov extended Nambu-Jona Lasinio model . . . . . . . . . . . . . . 79 
4.3 Quarks and mesons in the PNJL model . . . . . . . . . . . . . . . . . 80

4.4 Thermodynamic properties of the PNJL model . . . . . . . . . . . . . . . 84

4.5 Phase diagram of the $N_{f}=3$ PNJL model $\ldots \ldots \ldots \ldots$. . . . . 86

4.6 Elastic quark cross-sections in the PNJL model . . . . . . . . . . . . . . . 87

4.7 Transport coefficients in the PNJL model . . . . . . . . . . . . . . . . . . 91

4.8 Quark relaxation time in the PNJL model . . . . . . . . . . . . . . . . . 92

4.8.1 Method 1 for the quark relaxation time . . . . . . . . . . . 93

4.8.2 Method 2 for the quark relaxation time . . . . . . . . . . . . 95

4.8.3 Specific shear viscosity . . . . . . . . . . . . . . . . . . . . . . . 99

4.8.4 Electric conductivity and the dimensionless ratio of specific shear viscosity to the scaled conductivity . . . . . . . . . . . . . 101

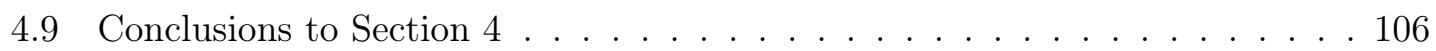

5 Transport properties of the QGP in the dynamical quasiparticle model with a CEP

5.1 Universal scaling approaching a critical end-point . . . . . . . . . . . . . 109

5.2 Extension of the quasiparticle DQPM-CP effective coupling constant for the inclusion of a CEP . . . . . . . . . . . . . . . . . . . . 109

5.3 Thermodynamic observables for finite temperature and chemical potential . 115

5.4 Approaching the CEP from the deconfined phase . . . . . . . . . . . . . 119

5.5 Transport coefficients of the QGP near the CEP . . . . . . . . . . . 122

5.5 .1 Specific viscosities . . . . . . . . . . . . . . . . . . 122

5.5.2 Electric, baryon and strange conductivities . . . . . . . . . . 125

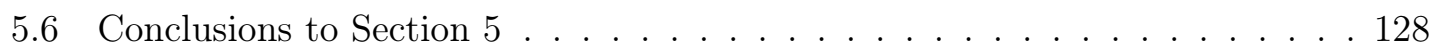

6 Conclusions and Outlook $\quad 129$

List of figures 132

$\begin{array}{ll}\text { List of tables } & 144\end{array}$

$\begin{array}{ll}\text { A Comparison study: constant isotropic cross sections } & 145\end{array}$

B Quark-(anti)quark scattering in the $N_{f}=3$ PNJL model $\quad 147$

B.0.1 Quark-quark scattering . . . . . . . . . . . . . . 147

B.0.2 Quark-antiquark scattering . . . . . . . . . . . . . . 148

C Application of the Event-Plane method to the PHSD 150

$\begin{array}{lr}\text { Abbreviations } & 152\end{array}$

$\begin{array}{lr}\text { References } & 153\end{array}$

$\begin{array}{lr}\text { Acknowledgements } & 169\end{array}$ 


\section{Introduction}

The exploration of fundamental properties of nuclear matter under the extreme conditions of high densities and high temperatures is a challenging topic, which can provide useful insights on many-bodies dynamics with strong-interactions. This is reflected both in the large number of theoretical studies on the subject and in the rich experimental program at Relativistic Heavy-Ion Collider (RHIC) (RHIC) and Large Hadron Collider (LHC) (LHC) energies as well as in the future experimental facilities such as the Facility for Antiproton and Ion Research (FAIR) in Darmstadt and the Nuclotron-based Ion Collider fAcility (NICA) in Dubna.

The majority of the experimental programs aim to explore the phase structure of strongly interacting nuclear matter, especially to determine the properties of a system in the vicinity of a phase transition from the confined hadronic phase to the deconfined phase known as quark-gluon plasma (QGP).

In such a hot and dense environment it is notoriously difficult to calculate equilibrium properties of the QGP from first principles, since the interaction is so strong, that a perturbative expansion in terms of the coupling constant is not applicable in the vicinity of the phase transition. Moreover, the study of QCD matter in heavy-ion collisions (HICs) can shed light on the mechanism of confinement, which is not fully understood from a theoretical point of view.

While the phase transition of the QGP matter, produced in collisions at the highest energies at LHC and RHIC and small baryon chemical potential, is conjectured to be a smooth crossover, lower energy collisions can explore higher baryon chemical potentials, where a possible first-order phase transition as well as critical endpoint (CEP) are predicted by some theoretical models.

The determination of basic transport coefficients, such as shear and bulk viscosities as well as electric, strange and baryon conductivities of the hot and dense QGP, is essential for the simulations of HICs based on hydrodynamical models, which use them as input parameters. The analysis of experimental data for elliptic flow conjectured that results can be reproduced by hydrodynamical simulations only with a small value for the shear viscosity over entropy density. The viscosity of a system quantifies its ability to restore local thermal equilibrium after being driven away from equilibrium by gradients in its macroscopic flow pattern. Viscous effects disappear as soon as the corresponding relaxation times approach zero. Small viscosities are governed by the short relaxation times or to strong interactions among the microscopic degrees of freedom.

The goal of this dissertation is to study the equilibrium as well as out-of-equilibrium properties of the strongly interacting QGP medium close to the phase transition under extreme conditions of high $T$ and high baryon densities by means of kinetic theory in the framework of effective models. We will explore first the thermodynamic and transport properties of the QGP near-equilibrium on the basis of the dynamical quasiparticle model (DQPM) in the region of moderate baryon chemical potential $\mu_{B} \geq 0.5 \mathrm{GeV}$. Furthermore, the DQPM 
allows to interpret the equation of state $(\mathrm{EoS})$ in terms of dynamical degrees of freedom and estimate the cross sections of the corresponding elastic and inelastic reactions, which are essential for the transport evolution.

Moreover, out-of equilibrium properties of the QGP and in particular, the effect of a $\mu_{B^{-}}$ dependence of thermodynamic and transport properties of the QGP have been studied within the Parton-Hadron-String-Dynamics (PHSD) transport approach, which covers the full evolution of the system including the partonic phase. The evolution of the partonic sector in the PHSD is extended by explicitly calculating the total and differential partonic scattering cross-sections based on the DQPM and evaluated at the actual temperature $T$ and baryon chemical potential $\mu_{B}$ in each individual space-time cell where partonic scattering take place. To explore the domain of high baryon chemical potential, where in the vicinity of the critical endpoint CEP located at $\left(T^{C E P}, \mu_{B}^{C E P}\right)=(0.100,0.960) \mathrm{GeV}$ we extend the DQPM adapting the critical behavior of the effective coupling constant by using the entropy density from the Polyakov extended Nambu-Jona Lasinio model (PNJL) close to the CEP.

The structure of the thesis is as follows. In Chapter 1 we begin with a discussion of the theoretical background of strong interactions and its applications to HICs. Chapter 2 is devoted to an exploration of thermodynamic and transport properties of QGP close to equilibrium at moderate baryon chemical potentials. In particular, we focus on the EoS and the speed of sound, as well as transport coefficients of the QGP, calculated on the basis of DQPM at finite $T$ and $\mu_{B}$.

In Chapter 3, we employ PHSD transport approach to study the QCD matter evolution during HICs at BES energies. We consider the evolution of the partonic systems at higher $\mu_{B}$ within an extended PHSD approach, which also incorporates partonic quasiparticles and their differential cross sections that depend not only on temperature $T$ (as in the previous PHSD versions) but also on the chemical potential $\mu_{B}$ explicitly. In particular, we discuss the 'bulk' observables in HICs at various energies - from AGS to RHIC - for symmetric $\mathrm{Au}+\mathrm{Au}$ and $\mathrm{Pb}+\mathrm{Pb}$ collisions. Furthermore, we enlarge our exploration to more sensitive to the partonic phase evolution observables, such as collective flow coefficients, where we focus on an impact of the $\mu_{B}$-dependencies of partonic cross sections on the flow coefficients.

In Chapter 4, we address the transport properties of QGP in the region of higher baryon chemical potentials, where the PNJL is employed. In that region, the influence of possible $\mathrm{CEP}$ and first-order phase transition is considered. Moreover, the basic transport coefficients are evaluated on the basis of kinetic theory in the relaxation-time approximation (RTA).

Finally, in Chapter 5 a phenomenological extension of the DQPM to large baryon chemical potentials $\mu_{B}$, including a region where a possible CEP and 1st-order phase transition, is considered. The EoS, the speed of sound as well as transport coefficients for $T>T_{c}$ are presented for a wide range of $\mu_{B}$ and $T$, employing two settings of the strange quark chemical potentials (I) $\mu_{q}=\mu_{u}=\mu_{s}=\mu_{B} / 3$ and (II) $\mu_{s}=0, \mu_{u}=\mu_{d}=\mu_{B} / 3$, which are of interest for hydrodynamical simulations. 


\title{
Theory of Strong Interactions
}

\author{
"No progress without paradox"
}

John Wheeler, 1985

Unlike quantum electrodynamics (QED), the theory of the strong interaction, i.e. quantum chromodynamics (QCD), cannot be easily cracked up by conventional - mainly perturbative methods - or tested via direct experimental measurements. In this Chapter we review the basic ingredients of a theoretical description of strong interactions and several experimental observables which shed light on the peculiar properties of QCD. In particular, the phenomena of the deconfined and chiral phase transitions in QCD and transport properties of the relevant degrees of freedoms are of great interest to the heavy-ion collision community. The full theoretical description of the evolution of the QCD medium in the heavy-ion collisions (HICs) involves phenomenological and effective models, which will be considered in the next Chapters. Moreover, during the HICs, the deconfined state of QCD matter - quark-gluon plasma (QGP) - which is supposed to consist of quarks and gluons, is created. It is predicted that the QGP has existed at $\sim 10$ microseconds after the Big Bang, however so far there is no solid experimental confirmation of it. Moreover it is important to note that the QGP created in HICs, in neutron stars or during the initial stages of the Universe, is under the influence of extreme conditions. Such conditions include high temperatures, strong magnetic fields, and high densities. These conditions result in a considerable change of thermodynamic and transport observables. The phase diagram of QCD can be considered in the $\left(T, \mu_{B}\right)$-plane. It has a rich structure, shortly discussed in following. The properties of the QCD medium under extreme conditions created during HICs, such as high temperature and high densities are addressed first in equilibrium in this Chapter and then out-of-equilibrium in the next Chapters.

\subsection{Fundamental properties of QCD}

QCD describes the strong interactions between quarks and gluons by means of an exchange of color charges. The complication as compared to QED is that the gluons are color-charged and therefore can interact between each-others. Moreover, this non-abelian nature of QCD encompasses important phenomena: asymptotic freedom and confinement. While in QED the virtual cloud screens the bare charge and results in a smaller charge at larger distances compared to the bare charge, in QCD the gluon self-interaction causes an opposite phenomenon - antiscreening. The phenomenon of charge screening in QED and antiscreening in QCD results in a running coupling. The running coupling constant can 


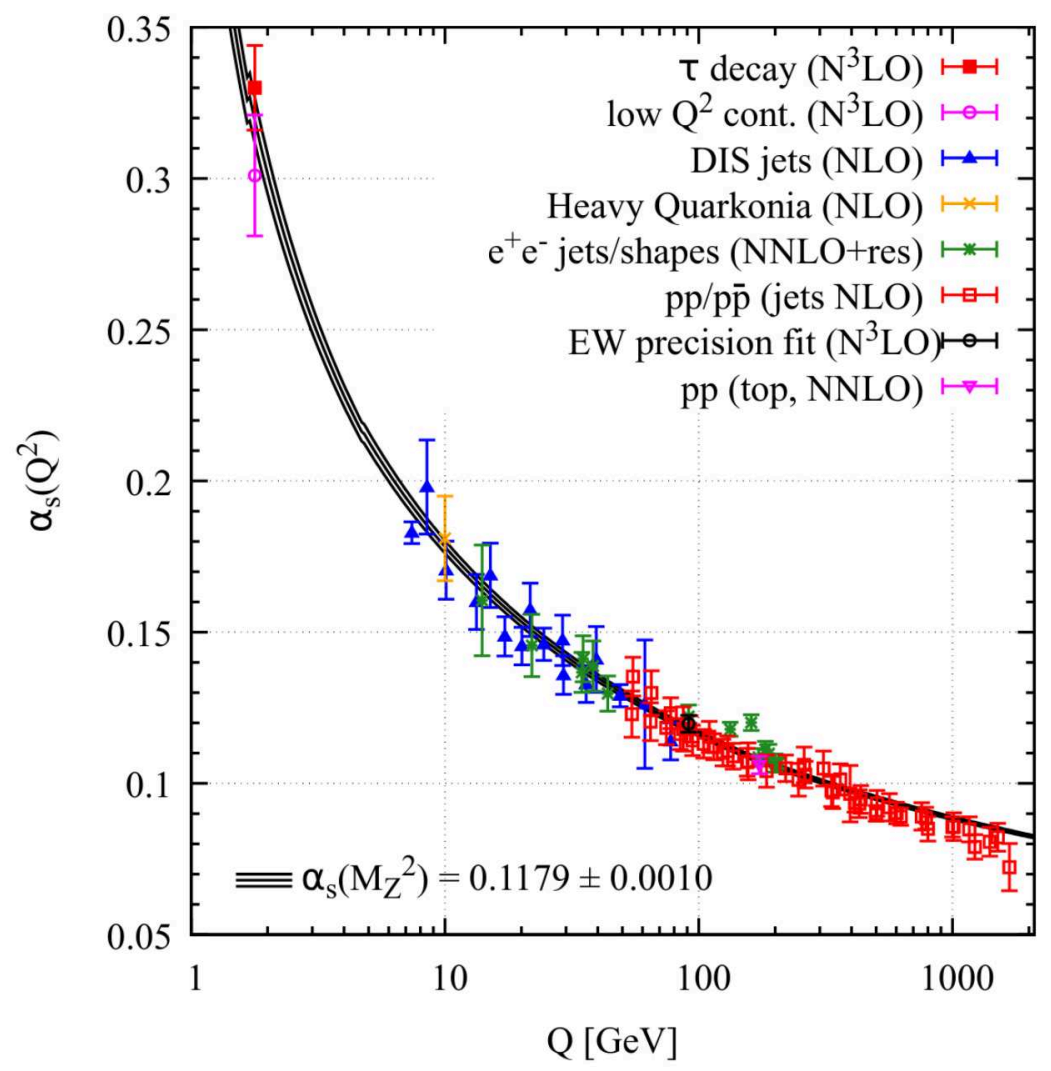

Figure 1.1: The running coupling of QCD as a function of the momentum scale $Q$. The figure is taken from [12].

be defined in terms of the $\beta$-function as follows from Refs. [10,11]:

$$
\alpha_{s}\left(Q^{2}\right)=\frac{\alpha_{s}\left(\mu^{2}\right)}{1+\frac{\alpha_{s}\left(\mu^{2}\right)}{12 \pi}\left(11 N_{c}-2 N_{f}\right) \ln \left(Q^{2} / \mu^{2}\right)} .
$$

Here $Q^{2}$ is the momentum transfer, $N_{c}=3$ denotes the number of colors, $N_{f}$ is the number of quark flavors relevant at the momentum scale $Q^{2}$ (in general there are $N_{f}=6$ quark flavors in the Standard Model). According to the Standard Model $\beta_{0}=11 N_{c}-2 N_{f}>0$ for QCD whereas it is negative for QED. Therefore, the coupling constant in QCD decreases with increasing $Q^{2}$ :

$$
\alpha_{s}\left(Q^{2}\right) \stackrel{Q^{2} \rightarrow \infty}{\longrightarrow} \frac{1}{\beta_{0} \ln \left(Q^{2} / \mu^{2}\right)} \stackrel{Q^{2} \rightarrow \infty}{\longrightarrow} 0 .
$$

This behaviour of a vanishing coupling strength at high $Q$ denotes the asymptotic freedom: at very high energies or small distances, quarks and gluons can be treated as free particles. As a consequence of asymptotic freedom, perturbative calculations become applicable at higher energies. Estimates via measurements of $\alpha_{s}$ for various $Q$ confirmed the running behaviour of $\alpha_{s}$. Figure 1.1 presents a summary of recent measurements of $\alpha_{s}$ as a function of $Q$.

Furthermore, one can simplify Eq. (1) by introducing the scale parameter of QCD 
$\Lambda_{Q C D}$ as follows

$$
\alpha_{s}\left(Q^{2}\right)=\frac{1}{\frac{11 N_{c}-2 N_{f}}{12 \pi} \ln \left(Q^{2} / \Lambda_{Q C D}^{2}\right)} .
$$

One can see that the perturbative expression of $\alpha_{s}\left(Q^{2}\right)$ diverges in the limit of small momentum transfer $Q$ and the parameter $\Lambda_{Q C D}$ corresponds the energy scale where $\alpha_{s}\left(\left|\mu^{2}\right|\right) \rightarrow$ $\infty$. The parameter $\Lambda_{Q C D}$ depends on $N_{f}$ and can be estimated by a comparison with experiments. For $N_{f}=3$ it is found to be $\sim 346 \mathrm{MeV}$ [13]. In the limit of small momentum transfer or large distances, the interaction becomes so strong that it is impossible to isolate a parton from a hadron, whereas it is cheaper to create a quark-antiquark pair. This phenomena is known as quark confinement. Confinement has been corroborated within the modern nonperturbative methods of lattice QCD (1QCD) calculations [14, 15]. However, due to the non-perturbative nature the confinement cannot be mathematically proven from first principles.

At small $Q^{2} \sim \Lambda_{Q C D}$ the perturbative expansion breaks down but alternative nonperturbative approaches can be applied in this domain. In particular, numerical simulations by means of the 1QCD approach [16] and more analytical methods such as the Functional Renormalisation Group (FRG) [17] and the functional approach via Dyson-Schwinger and Bethe-Salpeter equations [18] have been successfully used for the exploration of QCD in the non-perturbative low-energy regime.

To capture the basic features of QCD we consider classical Lagrangian density

$$
\mathcal{L}_{Q C D}=\sum_{q=u, d, s, \ldots} \bar{\psi}_{q, i}\left(i D_{i j}-m_{q} \delta_{i j}\right) \psi_{q, j}-\frac{1}{4} F_{\mu \nu}^{a} F_{a}^{\mu \nu},
$$

where $\psi_{q, i}\left(\overline{\psi_{q}, i} \equiv \psi^{\dagger} \gamma^{0}\right)$ is a quark field with fundamental color index $i$, which runs from $i=1$ to $N_{c}=3$, and $m_{q}=\operatorname{diag}\left(m_{u}, m_{d}, m_{s}, \ldots\right)$ is the quark-mass matrix, which is diagonal in the flavor space. The sum over $q$ runs over the quark flavors, $a$ is the adjoint color index, which represents combination of color charges carried by the gluon, running from $a=1$ to $N_{c}^{2}-1=8$. $F_{\mu \nu}^{a}$ denotes the gluon field strength tensor for a gluon with color index $a$. The gauge covariant derivative $D_{i j}$ in QCD reads

$$
D_{i j} \equiv \gamma^{\mu}\left(D_{\mu}\right)_{i j}=\gamma^{\mu} \partial_{\mu} \delta_{i j}-i g_{s} \gamma^{\mu} T_{i j}^{a} A_{\mu}^{a}
$$

Here, $\gamma^{\mu}$ are the Dirac gamma matrices, and $g_{s}=\sqrt{4 \pi \alpha_{s}}$ is the QCD coupling constant. The second part of the QCD Lagrangian density consists of the kinetic term, where the gluonic field strength tensor reads

$$
F_{a}^{\mu \nu}=\partial_{\mu} A_{\nu}^{a}-\partial_{\nu} A_{\mu}^{a}+g_{s} f^{a b c} A_{\mu}^{b} A_{\nu}^{c}
$$

Here $f^{a b c}$ are the structure constants of the $S U(3)$ group. As one can see applying Eq. (6) 
to the second term of Lagrangian density, there are 3-gluon and 4-gluon vertices with $f^{a b c}$, which are absent in case of an Abelian theory such as QED.

\subsection{Equilibrium properties of QCD matter: Lattice QCD}

The exploration of the QCD matter under extreme conditions requires non-perturbative methods since the conventional methods of perturbation theory are inapplicable at low energies, where the value of the coupling constant $g \gg 1$. In such a case, the main way of calculations in QCD are calculatiions of path integrals without involving an expansion in the coupling constant. The first formulation of the gauge theory on a space-time lattice was proposed by K. Wilson in 1974 [19]. The approach is based on a representation of the gauge field as a phase multiplier. The application of the lattice approach to quantum field theory is considered as a mathematical technique that enables a truncating of the ultraviolet divergences. At the same time, the lattice gauge theory method is the most natural way to calculate path integrals. In the lattice approach continuous four-dimensional space-time, on which the field functions are given, is replaced by a discrete space of finite size with a finite lattice step $a$. Therefore, the Lagrangian density has to be discretized and physical observables can be obtained from the correlation functions calculated on the lattice. The main components of the lattice discretization are

- node is a point on the lattice defined by the coordinates $x_{\mu}=a n_{\mu}$, where $n_{\mu}$ is a four-vector with components $\left(n_{x}, n_{y}, n_{z}, n_{t}\right)$. The number of nodes in different spatial directions is assumed to be the same and multiplied by the number of nodes in the temporal direction. In an isotropic lattice the step is the same in all directions. In the case of anisotropic lattices the step in spatial and temporal directions is different.

- link represents a rectilinear segment on the lattice connecting two neighboring nodes. A link is given by the coordinate $x_{\mu}$ of its origin and the direction of the corresponding axis in space. Each link is assigned a link variable $U_{\mu}(x)$, which is an $S U_{c}(3)$ matrix. The link associates lattice nodes with coordinates $x$ and $x+a \widehat{\mu}$, where is a unit vector in the direction $\widehat{\mu}$. When the direction of the link is reversed, the matrix $U_{\mu}(x)$ must be replaced by an hermitian-conjugate one. Moreover, the link variable $U_{\mu}(x)$ can be related to the continuous gluon field as $U_{\mu}(x)=\mathcal{P} \exp \left(i g_{0} \int_{x}^{x+a \widehat{\mu}} d z_{\mu} A_{\mu}(z)\right)$. It is important to note that the trace over products of links along any closed loop is gauge invariant.

- plaquette, or elementary square bounded by four links, is given by the coordinate of the node lying in its lower left corner and the positive directions of the attached links that form the square together with the nodes. The plaquette variable is written in the form:

$$
P_{\mu \nu}(n)=U_{\mu}(n) U_{\nu}(n+\widehat{\mu}) U_{\mu}^{\dagger}(n+\widehat{\nu}) U_{\nu}^{\dagger}(n)
$$

An illustration of the main components of the lattice discretization is given in Fig. 1.2.

Lattice calculations are based on a discretization of the path integrals of quantum field theory. The transition from Minkowski space to Euclidean space is performed by the 


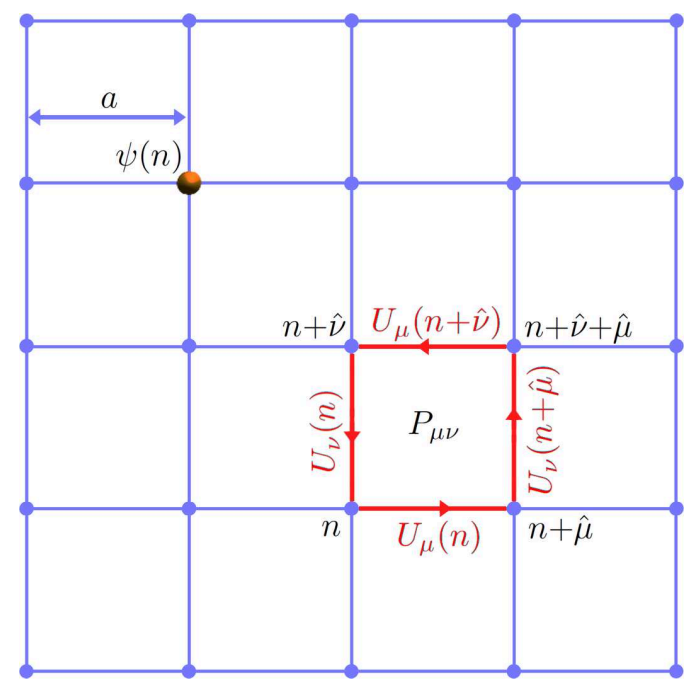

Figure 1.2: Sketch of the lattice discritization and its main ingredients: $\psi(n)$ is the fermion field defined on the sites, $U_{\mu}(n)$ is the link variable, and the plaquette $P \mu \nu(n)$.

Wick rotation, i.e. transforming the temporal component of the fields to the imaginary component:

$$
x_{0} \rightarrow-i x_{4}^{E}
$$

After the transition to imaginary time and imposing antiperiodic quantization conditions, the field theory in the space of $N$ dimensions turns into statistical mechanics in the space of $N-1$ dimensions. Thus the time in quantum-field theory is compared to the inverse temperature in statistical mechanics.

A key feature of the IQCD approach is the maintenance of gauge invariance without an introduction of additional nonphysical degrees of freedom. Preserving of the gauge invariance leads to significant adjustments, in particluar, by ensuring the vanishing masses of the gluons in the regularized theory.

\section{Thermodynamic observables}

One of the key components for the simulations of the evolution of QCD matter is the equation of state (EoS), which generally means the relation between the pressure and energy density and quark/baryon density, here however we refer to the EoS as the $T$ and $\mu_{B^{-}}$dependence of thermodynamic quantities such as pressure $p$ and energy density $\epsilon$. The thermodynamic quantities can be evaluated within the IQCD approach, as well as within other non-perturbative methods, and within a variety of QCD-like models such as the (P)NJL model, the Linear sigma model, the on-shell and off-shell quasiparticle models etc.

Now we focus on results for the thermodynamic observables from the lQCD calculations in equilibrium.

According to the statistical mechanics all thermodynamic quantities of the system with hamiltonian $H$ at finite $T$ in equilibrium can be derived from the partition function

$$
Z(\beta)=\operatorname{Tr} \exp (-\beta H)
$$




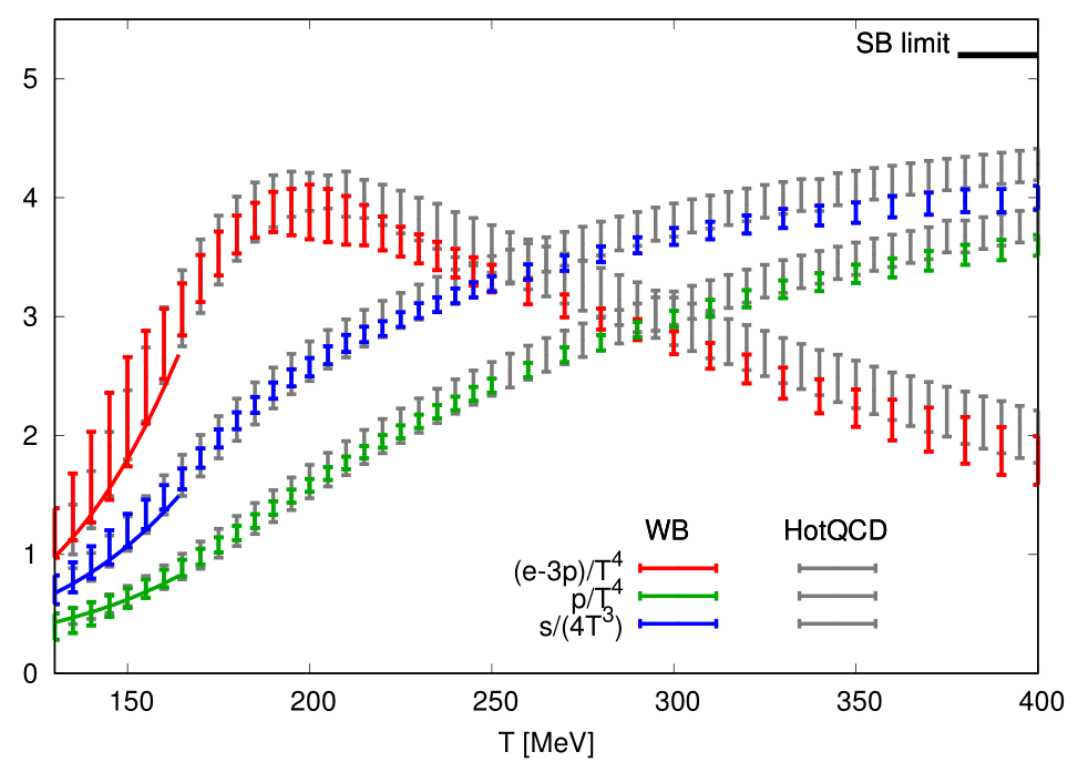

Figure 1.3: The dimensionless thermodynamic observables as a function of temperature for $N_{f}=2+1$. The gray points are from the HotQCD collaboration [21], while the colored ones are from the WB collaboration [22]. The figure is taken from [16].

with $\beta=1 / T$ being inverse temperature. The partition function can be expressed as a path integral in the Euclidean space containing the corresponding Lagrangian density [20]:

$$
Z(\beta, V)=\int D[A] D[\psi] D[\bar{\psi}] \exp \left(-\int_{0}^{\beta} d x_{4} \int_{V} d^{3} x \mathcal{L}_{E}(A, \psi, \bar{\psi})\right)
$$

Here first integration runs over a finite size with a thickness determined by the inverse temperature. The integration in lattice formulation is replaced by the sum over a finite lattice size $L$. The integration over the quark fields can be performed analytically, while the partition function for the gauge fields can only be calculated within Monte-Carlo methods. The continuum extrapolated results are obtained after performing thermodynamic $L \rightarrow \infty$ and continuum $a \rightarrow 0$ limits.

From the partition function the main thermodynamic observables can be deduced. For instance, the pressure and energy density reads

$$
\begin{gathered}
P=T\left(\frac{\partial \ln Z}{\partial V}\right)_{T}, \\
\epsilon=-\frac{1}{V}\left(\frac{\partial \ln Z}{\partial T^{-1}}\right)_{V} .
\end{gathered}
$$

Other thermodynamic observables can be obtained through Euler relation for the grandcanonical ensemble. In lattice simulations a lattice with a finite number of time steps $N_{4}$ is considered. A time grid with a finite number of steps in time $N_{4}$ is used. Thus the temperature of the system is defined as $T=\frac{1}{N_{4} a}$. The size of the grid in the space directions $N=N_{1}=N_{2}=N_{3}$ should be infinitely large. In practice it is finite but satisfies the ratio $N \gg N_{4}$. In the limit $N_{4} \rightarrow \infty$ the system corresponds to the field theory at 
zero temperature.

The continuum extrapolated results for the scaled interaction measure, entropy density and pressure are displayed in Fig. 1.3. As one can see there is a good comparison between the results obtained from the HotQCD collaboration [21] (gray points), and from the WB collaboration [22] (colored points). The non-interacting Stefan-Boltzmann limit of massless quarks and gluons for high $T$ is depicted for the pressure and the scaled entropy. The lines at low temperature correspond to the HRG model predictions. In the vicinity of phase transition the thermodynamic observables undergo a rapid increase with the increasing temperature due to the increasing number of dynamical degrees of freedom.

While at vanishing baryon chemical potential 1QCD calculations provide smooth results for the thermodynamic observables for a large range of temperature, a direct calculation of the EoS at non-zero baryon and other chemical potentials is not yet established due to the well-known sign problem, which refers to the fact that the fermion determinant becomes a complex function for finite quark chemical potentials. However, at small chemical potentials this can be bypassed by employing a Taylor expansion of the thermodynamic potential in terms of baryon, electric charge and strangeness chemical potentials [23, 24]. The resulting EoS has been obtained for chemical potentials up to $\mu_{B} / T \lesssim 3.0$ [21,22]. Recently, a new expansion scheme has been proposed, which covers regions of moderate chemical potentials up to $\mu_{B} / T \leq 3.5$ [25].

\subsection{Symmetries and phases of QCD}

Global symmetries and associated conserved currents and charges play an important role in thermodynamics and affect the phase structure of QCD. QCD possesses many symmetries, exact and approximate, which leads to a rich phase structure that will be discussed below.

First the QCD Lagrangian is invariant under local color transformations $\omega(x) \in S U(3)_{c}$

$$
\begin{array}{r}
\omega(x)=\exp \left(i g \theta^{a}(x) \lambda^{a} / 2\right), \\
\psi_{q}(x) \rightarrow \psi_{q}^{\prime}(x)=\omega(x) \psi_{q}(x), \\
A_{\mu}(x) \rightarrow A_{\mu}^{\prime}(x)=\omega(x)\left(A_{\mu}(x)+\frac{1}{g} \partial_{\mu}\right) \omega^{\dagger}(x),
\end{array}
$$

where $\theta^{a}$ denotes arbitrary real numbers and $\lambda^{a}$ are Gell-Mann matrices. The local color $S U(3)$ symmetry results in a similar strength for the quark-quark and gluon-gluon interaction, which is governed by the coupling constant $\alpha_{s}$. Nevertheless, in the region of high chemical potential and low temperature, the color $S U(3)$ symmetry is spontaneously broken; this domain corresponds to the color superconductive phase. Moreover, the QCD Lagrangian obeys discrete $C, P, T$ - and combined $C P T$ symmetries.

Later on we consider global symmetries of QCD with associated transformations $V$, where $V \in S U\left(N_{f}\right)$ or $U\left(N_{f}\right)$.

The two light quarks as well as the strange quark have relatively small masses, compared to hadron scales $\left(\approx m_{N}=0.938 \mathrm{GeV}\right)$. Therefore, the approximation of vanishing light and/or strange quark masses can be considered. In this case the QCD Lagrangian has a 
global flavor symmetry

$$
S U\left(N_{f}\right)_{V} \otimes S U\left(N_{f}\right)_{A} \otimes U(1)_{V} \otimes U(1)_{A},
$$

where $N_{f}$ denotes the number of quark flavors and indices $V$ and $A$ denote 'vector' and 'axial(-vector)' w.r.t. to the Lorentz transformation properties of the associated conserved currents. By increasing $N_{f}$ the global symmetry (16) becomes more broken by the heavy quark masses.

In the case of $N_{f}=2$, the quark masses are quite small, in the limit $m_{u} \approx m_{d}$, there is a corresponding $S U(2)_{V}$ isospin symmetry, with corresponding transformations

$$
\psi_{q} \rightarrow e^{-\frac{i}{2} \theta_{k} \tau_{k}} \psi_{q}
$$

If we consider the limit of quark masses $m_{u} \approx m_{d} \approx 0\left(m_{u} \approx 5 \mathrm{MeV}\right.$ and $\left.m_{d} \approx 9 \mathrm{MeV}\right)$ there is a associated $S U(2)_{A}$ chiral symmetry.

$$
\psi_{q} \rightarrow e^{-\frac{i}{2} \gamma_{5} \theta_{k} \tau_{k}} \psi_{q}
$$

The $U_{V}(1)$ symmetry is independent of the quark mass. It corresponds to a phase change for all quark species

$$
\psi_{q} \rightarrow e^{-i \alpha} \psi_{q}
$$

The baryon number is an exactly conserved number of the strong interaction, which is also used to specify types of hadrons.

Furthermore, for $N_{f}=3 \mathrm{QCD}$ in the limit of $m_{u}=m_{d}=m_{s}$ involves a chiral symmetry $S U(3)_{V} \otimes S U(3)_{A}$, which is equivalent to $S U(3)_{L} \otimes S U(3)_{R}$. Nevertheless, $S U(3)_{A}$ as well as $S U(2)_{A}$ are spontaneously broken, which implies an existence of massless NambuGoldstone bosons: three bosons for the $N_{f}=2-\pi^{ \pm}, \pi^{0}$ and eight bosons for $N_{f}=3$ $K^{ \pm}, K^{0}, \bar{K}^{0}, \eta$. Due to the finite quark masses, these so-called pseudo-Goldstone bosons have finite masses, however, much lower than other hadrons. The order parameter of the chiral transition is the chiral condensate $\langle\psi \bar{\psi}\rangle$, which drops to 0 in a phase with the exact chiral symmetry, while $\langle\psi \bar{\psi}>\neq 0$ in the phase with the broken chiral symmetry.

The deconfinement transition - in a limit of infinite quark masses - is related to spontaneous symmetry breaking of the $Z_{3}$ center symmetry of $S U(3)_{c}$ [27]. In QCD for infinite quark mass the order parameter of the deconfinement phase transition is the Polyakov loop, expressed in terms of the Euclidean temporal component of the gauge field $A_{4}^{E}$ as

$$
\begin{array}{r}
<\Phi>=\frac{1}{N_{c}} \operatorname{Tr}_{\mathrm{c}} L_{x}, \\
L_{x}=\mathcal{P} \exp \left[i \int_{0}^{1 / T} d \tau A_{4}^{E}(x, \tau)\right],
\end{array}
$$

where $\mathcal{P}$ is the path-integral ordering operator, and the trace $\operatorname{Tr}_{\mathrm{c}}$ is taken in the color space. Global center $Z_{N_{c}}$ symmetry, related to the transformations

$$
L_{x} \rightarrow z^{k} L_{x}, z^{k}=e^{i 2 \pi k / N c}
$$



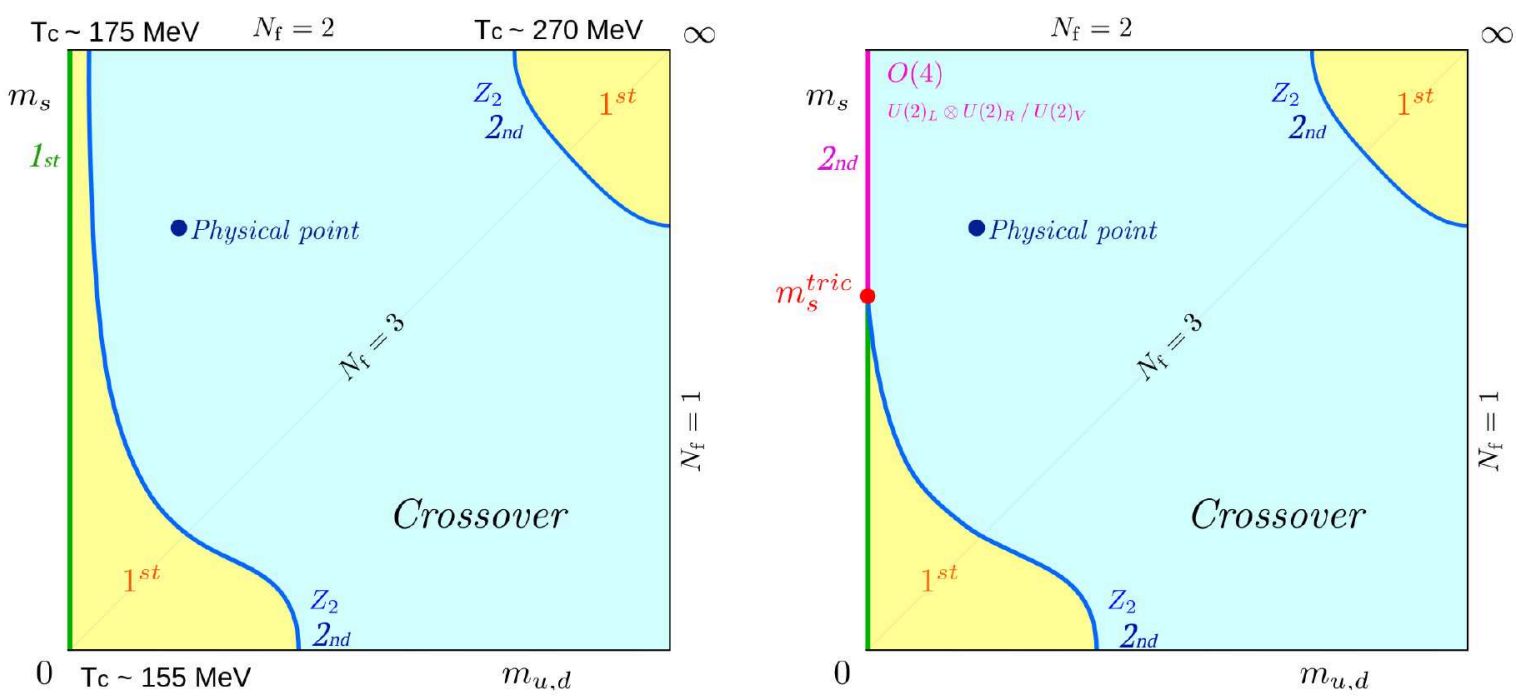

Figure 1.4: Theoretical sketch of the order of the thermal QCD transition as functions of a light quark mass $m_{u, d}$, and a heavier strange quark mass, $m_{s}$, at vanishing baryon chemical potential. Every point of the plot corresponds to a phase boundary, with an implicitly associated (pseudo-)critical temperature $T_{c}\left(m_{u, d}, m_{s}\right)$. Two scenarios are considered for $N_{f}=2, m_{u, d}=0$ : first-order (left) and second-order (right). The figure is adapted from [26].

which multiplies all the Polyakov lines $L_{x}$ by the same phase factor $z^{k}$. In the pure $S U\left(N_{c}\right)$ gauge theory this is an exact symmetry of the Yang Mills action.

Its expectation value is related to a change in the free-energy occurring adding an isolated heavy quark into the system:

$$
<\Phi>=e^{-\Delta F_{Q} / T}
$$

$<\Phi>$ vanishes in the confined phase, since adding an isolated color charge requires an infinite amount of energy; it is non-zero in the deconfined phase, where this suppression of colored states is absent. Light dynamical quarks influence an explicit breaking of the $Z_{3}$ symmetry, nevertheless the Polyakov loop, in light of its physical meaning, remains a useful quantity to identify the different phases predicted by theory.

The chiral and confinement phase transitions - governed by the chiral and center symmetries - are most important for the exploration of QGP properties at finite $T$ and $\mu_{B}$. However, it has been shown by $1 \mathrm{QCD}$ calculations that the two transitions have different critical temperatures. As it has been discussed above, both symmetries are broken at $\mu_{B}=0$ with finite quark masses, however, the chiral symmetry is restored for vanishing quark masses, whereas the center symmetry is restored for infinite heavy quarks. Various ways of the influence of quark masses on the order of chiral and deconfinement phase transitions can be summarised in a so-called Columbia plot presented in Fig. 1.4 at vanishing baryon chemical potential. As one can see the number of active quark flavors, as well as quark masses, influence not only the order of the phase transition but the critical temperature $T_{c}$ and possibly thermodynamic observables as well. 


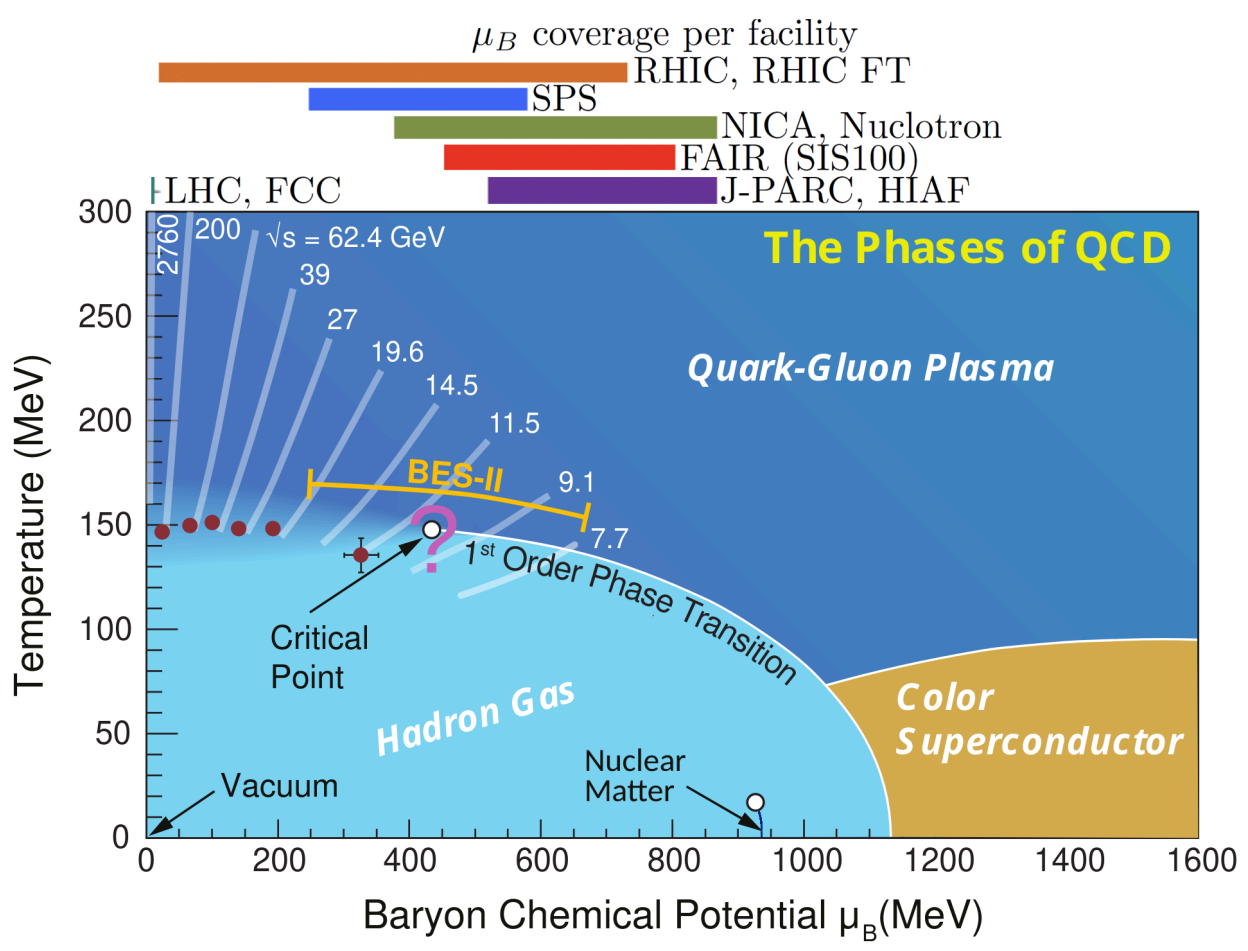

Figure 1.5: Schematic phase diagram of QCD in the temperature $T$ and baryon chemical potential $\mu_{B}$ plane. Horizontal lines on the top panel represent the intervals of $\mu_{B}$ covered in correspondent experimental facilities. The figure is based on [30].

\subsection{Phase diagram of QCD matter}

Usual cold nuclear matter $T=0$ is considered at $\mu_{B}=m_{N}=938 \mathrm{MeV}$. In QCD at low temperatures and baryon chemical potentials, quarks and gluons are bound into hadrons, therefore the lower left part of the diagram corresponds to a hadron resonance gas. At low temperatures but high baryon chemical potentials, the nuclear matter enters a color superconductive phase. It is believed that this phase can be possibly realized in the center of neutron stars [28]. On the other hand, at high temperatures or/and finite baryon chemical potentials, quarks and gluons become deconfined, and a QGP phase is formed [29].

While an examination of the QGP, created in the central interaction volume at ultrarelativistic energies at the Large Hadron Collider (LHC) or the Relativistic Heavy-Ion Collider (RHIC), has been performed at almost zero baryon chemical potential $\left(\mu_{B} \approx\right.$ 0 ) the most intriguing region of moderate and high baryon chemical potential and finite temperature remains unexplored experimentally. On the theory part, there are big discrepancies between various model predictions, especially concerning the phase structure, while first principle approaches for finite $T$ and $\mu_{B}$ become notoriously difficult. However, some of the quantitative results from the effective models are in agreement. It is known from IQCD calculations that the phase transition at vanishing baryon chemical is a rapid crossover [31]. However, it is predicted from the various QCD-like models that the order of the phase transition can change at finite $\mu_{B}$. It has been argued that at $T \approx 0$ and large baryon chemical potential $\mu_{B}>m_{N}$ the phase transition should be of first-order. Therefore, with a decrease of $\mu_{B}$ the possible first-order transition line should terminate 


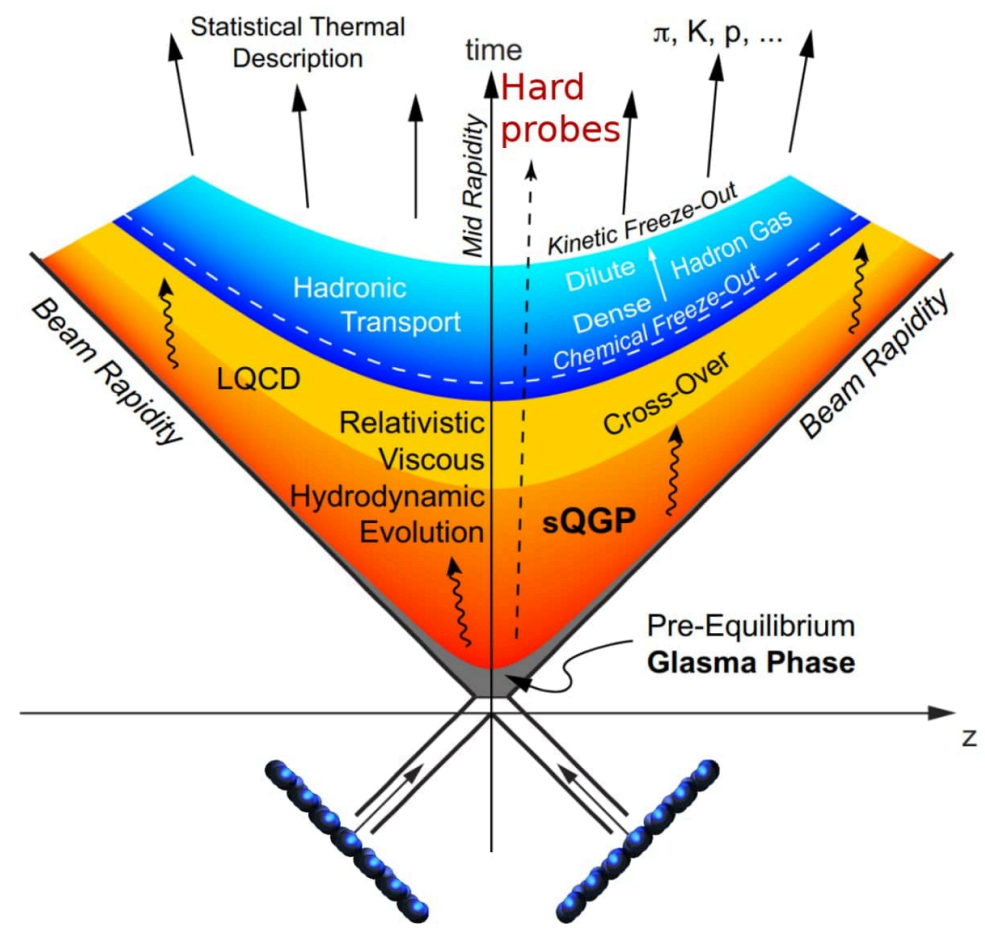

Figure 1.6: Qualitative spacetime evolution of a relativistic heavy-ion collision. The nuclei (blue discs) propagate along the z-axis at the speed of light and collide at $z=t=0$. The QGP(orange region) medium forms at proper time $\tau \sim 1 \mathrm{fm} / \mathrm{c}$ and converts to a hadron resonance gas (blue region) around $\tau \sim 10 \mathrm{fm} / \mathrm{c}$. The figure is based on [35].

at a critical endpoint (CEP), where the transition is of second-order, and with a further decrease of $\mu_{B}$ the phase transition becomes a crossover. Such a scenario is depicted in the theoretical sketch of the QCD phase diagram, presented in Fig. 1.5. It is worth pointing out that the conjectured QCD phase diagram has a few similarities with the phase diagram of water, which usually is presented as a function of pressure $P$ and temperature $T$.

\subsection{Dynamical properties of QCD matter: Modelling the heavy-ion collisions}

Many properties of QGP matter have been established by experimental observations in ultrarelativistic HICs at RHIC and LHC. Therefore, the QGP-matter has to be explored within off-equilibrium methods, while well defined QGP properties in equilibrium are used as well. Such a consistent description of all stages of HICs within a single approach has to provide a unifying explanation of many physical phenomena. The most prominent are jet quenching [32], multi-particle long-range flow correlations, strangeness 'enhancement' [33], quarkonium suppression and regeneration [34], open heavy-flavor diffusion and electromagnetic radiation.

We begin with lead-lead collision, where the Lorentz contracted nuclei are moving along the $\mathrm{z}$ axis, while a QGP system is created in the central overlap area. Figure 1.6 illustrates the qualitative space-time evolution of a relativistic HICs, where the three main 
stages can be considered: an early non-equilibrium stage, a further expansion stage, and a final freeze-out stage. Such a separation allows to apply different advanced theoretical approaches at every stage. Therefore, the simulation of HICs can be performed via conventional transport and hydrodynamical models and used to further explore the properties of the strongly interacting medium.

When the hot and dense matter is created, there main degrees of freedom are quarks and gluons, which participate in elastic and inelastic scatterings until the system reaches thermal equilibrium or hadronization. The first stage, which provides the initial conditions, such as distribution of the deposited energy density and pressure, for further hydrodynamic expansion is the least known, however, it can be described by simple geometric models such as Glauber's Monte-Carlo, in which the underlying interactions are enclosed in an inelastic nucleon-nucleon cross section. More ab-initio descriptions such as the color glass condensate (CGC), in which a collision is viewed in terms of parton degrees of freedom (mainly gluons in the appropriate kinematic mode for RHIC and LHC) and QCD interactions are actively being developed at present. Further studies of the high density regime of nuclear gluons will be possible in future at the electron-ion collider (EIC), which is currently planned to be built in the United States.

In the CGC picture the initial scattering results in a dense system of strong classical color fields, the so-called Glasma, which quickly thermalizes. Less than $1 \mathrm{fm} / \mathrm{s}$ is required or assumed for the system to turn into a nearly perfect fluid; the expansion of which can be described by relativistic viscous hydrodynamics.

During the second stage the fireball undergoes an expansion due to the partial gradients in space. Since the system is thermalized the space-time evolution might be described by relativistic viscous hydrodynamics. Due to the near-ideal liquid nature of the QGP, the initial geometric anisotropy is effectively converted into a momentum anisotropy of the final particles. Event fluctuations lead in final states to significant higher-order harmonics (triangular flow) of the azimuthal distribution of particles, in addition to the second order harmonic (elliptic flow). Their systematic measurement made it possible to restrict the calculations of the model for the initial state and conjecture transport properties of the QGP both at RHIC and LHC. Moreover, this evolution provides an input for medium modifications of hard probes, although a better description of the interplay of hard probes and the dense medium evolution is required.

The expansion of the system causes cooling and a hadronization occurs when the system reaches a critical energy density $\epsilon_{c} \approx 0.5 \mathrm{GeV} / \mathrm{fm}^{3}$ which corresponds to a (pseudo)critical temperature $T_{c} \sim 155 \mathrm{MeV}$ for $\mu_{B} \approx 0$. After hadronization, the scattering rate and density of the system decrease rapidly and the kinetic description becomes more relevant. This third stage can be described by hadronic transport models such as UrQMD [36,37], HSD [38], AMPT [39], SMASH [40]. Nevertheless, conventional transport models can be employed to describe both pre-equilibrium and equilibrium stages, where the space-time evolution is coupled to a mechanism of particle production. Moreover, hybrid models, such as URQMD+vHLLE [41] and MUSIC+UrQMD [42], as well as some advanced transport approaches, such as AMPT [39] and Parton-Hadron-String-Dynamics (PHSD) [1,43] can 
provide the whole evolution of HICs, including the QGP phase. We will discuss the PHSD transport approach and it is application to the HICs in the BES energy range in Chapter 3.

The measured relative numbers of hadrons show that a chemical freezing occurs at a temperature $T_{c h}$, which is very close to the hadronization temperature at almost zero $\mu_{B}$. Subsequently, hadrons continue to scatter elastically until they reach the kinetic freezing temperature $T_{f o}$, where they split and freely fly to the detectors. 


\title{
Dynamical quasiparticle model of QGP at finite $\mu_{B}$
}

\author{
"Just because things get a little dingy at the subatomic level \\ doesn't mean all bets are off" \\ Murray Gell-Mann
}

In this Chapter, we proceed with the thermodynamic quantities and transport properties of the QGP at finite temperature $T$ and moderate baryon chemical potentials $\mu_{B} \leq 0.5$ $\mathrm{GeV}$. In a hot and dense environment it is notoriously difficult to assess the microscopic properties of the QGP using first principles [44]. The expansion and dilution of the quarkgluon medium produced in nuclear collisions can be described by relativistic viscous hydrodynamics, which includes the transport coefficients in the dissipative part. Although the hydrodynamic equations provide a macroscopic description of the relativistic fluid behaviour, transport coefficients have to be determined by an underlying microscopic theory. They provide information about the interactions inside the medium. An alternative to ab initio lattice QCD calculations approach, there are effective models on the basis of 'resummed' propagators and couplings, which can describe microscopic properties of the QGP in a wide range of baryon chemical potentials.

In this Chapter we discuss the main properties of the dynamical quasiparticle model (DQPM), initially developed in Refs. [45-47]. We also show basic thermodynamic observables as well as the transport properties of the QCD medium at moderate baryon chemical potentials $\mu_{B} \leq 0.5 \mathrm{GeV}$, where we assume that in this region the phase transition between the hadronic matter and the quark-gluon plasma is a smooth crossover. We evaluate transport coefficients within the relaxation-time approximation (RTA) on the basis of interaction rates employing differential cross sections as functions of temperature and baryon chemical potential. An extension of the DQPM to the region of high baryon densities, where the phase transition is expected to be of first-order, will be considered in Chapter 5. Considering the transport coefficients and the EoS of the QGP phase, we compare our results with various results from the literature, where transport coefficients of the QGP have been examined on the basis of effective models dominantly at zero or small chemical potentials [48-55]. In this case 1QCD calculations can serve as a guideline at vanishing chemical potential. 
Part of the results demonstrated below have been presented in publications $[1,2,6]$ or are based on those. ${ }^{1}$

\subsection{Basics of the off-shell quasiparticle model}

For a complete description of the QGP dynamics one needs first to evaluate the microscopic properties of the relevant degrees of freedom such as the effective masses and widths of the partonic propagators as well as elastic cross-sections. In the range of temperatures $T \geq T_{c}$ and moderate baryon chemical potentials, one can rely on estimates from effective models. Here we consider essential features of the QGP medium in terms of strongly interacting quarks and gluons as given by the dynamical quasiparticle model DQPM $[45-47,56]$.

The DQPM reproduces the EoS of the partonic system above the deconfinement temperature $T_{c}$ from lattice QCD and provides reasonable estimates for the QGP transport coefficients, which - as will be shown later - agree well with the IQCD calculations at $\mu_{B}=0$. In the DQPM, the quasiparticles are characterized by dressed propagators,

$$
\Delta_{i}(\omega, \mathbf{p})=\frac{1}{\omega^{2}-\mathbf{p}^{2}-\Pi_{i}}
$$

with complex self-energies $\Pi_{i}=m_{i}^{2}-2 i \gamma_{i} \omega$. Here the real part of the self-energies is related to dynamically generated thermal masses $m_{i}$, while the imaginary part provides the information about the lifetime and interaction rates of the partons (interaction widths), using $\omega$ for energy (where $m_{i}$ and $\gamma_{i}$ are the thermal mass and the width of a parton). In the DQPM the spectral functions of the quasiparticles $\rho_{i}=-2 \operatorname{Im} \Delta_{i}$ (where $i=q, \bar{q}, g$ ) are no longer $\delta$-functions in the invariant mass squared. The spectral functions of interacting quasiparticles can be written in the lorentzian form, [46]

$$
\begin{aligned}
\rho_{i}(\omega, \mathbf{p})= & \frac{\gamma_{i}}{\tilde{E}_{i, \mathbf{p}}}\left(\frac{1}{\left(\omega-\tilde{E}_{i, \mathbf{p}}\right)^{2}+\gamma_{i}^{2}}-\frac{1}{\left(\omega+\tilde{E}_{i, \mathbf{p}}\right)^{2}+\gamma_{i}^{2}}\right) \\
& =\frac{4 \omega \gamma_{i}}{\left(\omega^{2}-\mathbf{p}^{2}-m_{i}^{2}\right)^{2}+4 \gamma_{i}^{2} \omega^{2}} .
\end{aligned}
$$

In Eq. (25) the off-shell energy $\tilde{E}_{i, \mathbf{p}}=\sqrt{\mathbf{p}^{2}+m_{i}^{2}-\gamma_{i}^{2}}$ is introduced, with $m_{i}, \gamma_{i}$ being the pole mass and width, separately for quarks, antiquarks and gluons $(i=q, \bar{q}, g)$. The spectral function is a real function, odd in $\omega$ and for all $\mathbf{p}$ it fulfills the sum rule

$$
\int_{-\infty}^{\infty} \frac{d \omega}{2 \pi} \omega \rho_{i}(\omega, \mathbf{p})=1
$$

\footnotetext{
$1 \quad$ This work has been done in collaboration with Pierre Moreau (Goethe U.), who conducted the evaluation of the differential and total cross sections. Programs for the calculations of the relaxation times, transport coefficients and plotting routines have been developed by the author of the present thesis. The comparison to the diffusion coefficients from the RTA and the Chapman-Enskog methods has been performed in collaboration with Jan Fotakis (Goethe U.), who provided the results for the Chapman-Enskog method.
} 

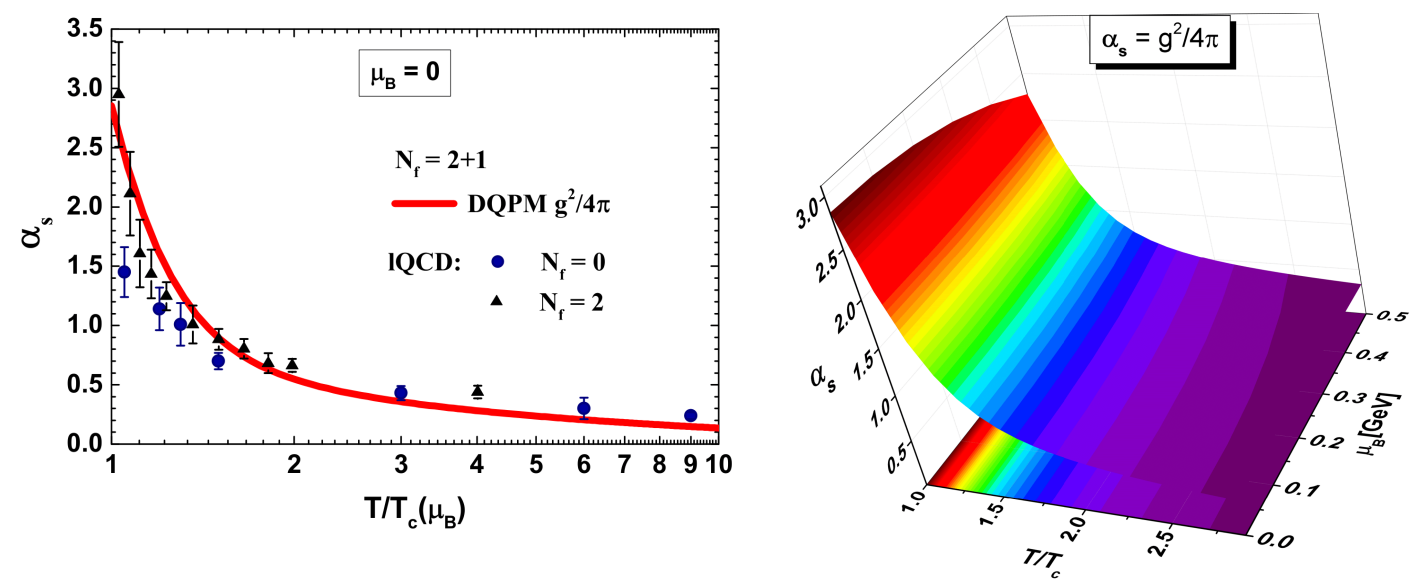

Figure 2.1: DQPM effective running coupling $\alpha_{s}=g^{2} /(4 \pi)$ as a function of the scaled temperature $T / T_{c}\left(\mu_{\mathrm{B}}\right)$ at vanishing chemical potential $\mu_{\mathrm{q}}=\mu_{\mathrm{B}}=0$ (left) and for moderate baryon chemical potentials $\mu_{\mathrm{B}} \leq 0.5 \mathrm{GeV}$ obtained within the 'scaling hypothesis' (2.1) (right) [1,57]. The lattice results for quenched QCD, $N_{f}=0$, (blue circles) are taken from Ref. [58] and for $N_{f}=2$ (black triangles) are taken from Ref. [59]. This figure is adopted from [6].

In principle, one can first determine the spectral functions and then the quasiparticle (retarded) propagators can be expressed in the Lehmann representation via the spectral functions as:

$$
\Delta_{i}(\omega, \mathbf{p})=\int_{-\infty}^{\infty} \frac{d \omega^{\prime}}{2 \pi} \frac{\rho_{i}\left(\omega^{\prime}, \mathbf{p}\right)}{\omega-\omega^{\prime}}=\frac{1}{\omega^{2}-\mathbf{p}^{2}-m_{i}^{2}+2 i \gamma_{i} \omega}
$$

One can see that from Eq. (27) the real part of the retarded self-energy is $m_{i}^{2}$. The main properties and dynamics of the quasiparticle model are governed by the coupling of the QCD matter. A distinctive feature of the DQPM is the determination of the coupling constant by using the IQCD entropy density at $\mu_{B}=0$. One can start from zero baryon chemical potential $g^{2}\left(T, \mu_{B}=0\right)$, while generally the coupling constant depends on both temperature and baryon chemical potential. In the DQPM the coupling constant at $\mu_{B}=$ 0 is parameterized employing the entropy density $s\left(T, \mu_{\mathrm{B}}=0\right)$ from the lattice $\mathrm{QCD}$ calculations provided by the BMW collaboration in Refs. $[22,60]$ in the following way:

$$
g^{2}\left(T, \mu_{\mathrm{B}}=0\right)=d \cdot\left[\left(s(T, 0) / s_{\mathrm{SB}}^{\mathrm{QCD}}\right)^{e}-1\right]^{f},
$$

with the Stefan-Boltzmann entropy density $s_{\mathrm{SB}}^{\mathrm{QCD}} / T^{3}=19 \pi^{2} / 9$ and the dimensionless parameters $d=169.934, e=-0.178434$ and $f=1.14631$. We note that the DQPM has been used to explore the crossover region in the phase diagram by introducing an effective coupling constant, which depends on the baryon chemical potential [1,47]. In this region of moderate baryon chemical potentials the basic thermodynamic observables, estimated by the IQCD have a smooth $\mu_{B}$-dependence. Therefore, we expect a similar behaviour for the effective coupling.

At finite baryon chemical potential $\mu_{\mathrm{B}}$ the effective coupling is obtained by employing the 'scaling hypothesis' introduced in Ref. [45]. It assumes that $g^{2}$ is a function of the ratio 
of the effective temperature

$$
T^{*}=\sqrt{T^{2}+\mu_{q}^{2} / \pi^{2}}
$$

(where the quark chemical potential is defined as $\mu_{q}=\mu_{u}=\mu_{s}=\mu_{B} / 3$ ) and the $\mu_{\mathrm{B}^{-}}$ dependent critical temperature $T_{c}\left(\mu_{\mathrm{B}}\right)$ defined as in Ref. [47]:

$$
T_{c}\left(\mu_{B}\right)=T_{c}(0) \sqrt{1-\alpha \mu_{B}^{2}}
$$

where $T_{c}(0)$ is the critical temperature at vanishing chemical potential $\left(T_{c}(0) \approx 0.158 \mathrm{GeV}\right)$ and $\alpha=0.974 \mathrm{GeV}^{-2}$. Thus, the DQPM effective coupling constant $g_{D Q P M}^{2}\left(T, \mu_{\mathrm{B}}\right)$ reads

$$
g_{D Q P M}^{2}\left(T, \mu_{\mathrm{B}}\right) \equiv\left\{\begin{array}{l}
\mu_{\mathrm{B}}=0: g^{2}\left(T, \mu_{\mathrm{B}}=0\right) \\
\mu_{\mathrm{B}}>0: g^{2}\left(T_{\text {scale }}\left(T, \mu_{\mathrm{B}}\right)\right) \\
\text { with } \mathrm{T}_{\text {scale }}=\frac{\mathrm{T}^{*}}{\mathrm{~T}_{\mathrm{c}}\left(\mu_{\mathrm{B}}\right) / \mathrm{T}_{\mathrm{c}}(0)}
\end{array}\right.
$$

The $\left(T, \mu_{\mathrm{B}}\right)$-dependence of the DQPM running coupling $\alpha_{s}=g_{D Q P M}^{2} /(4 \pi)$ is shown in
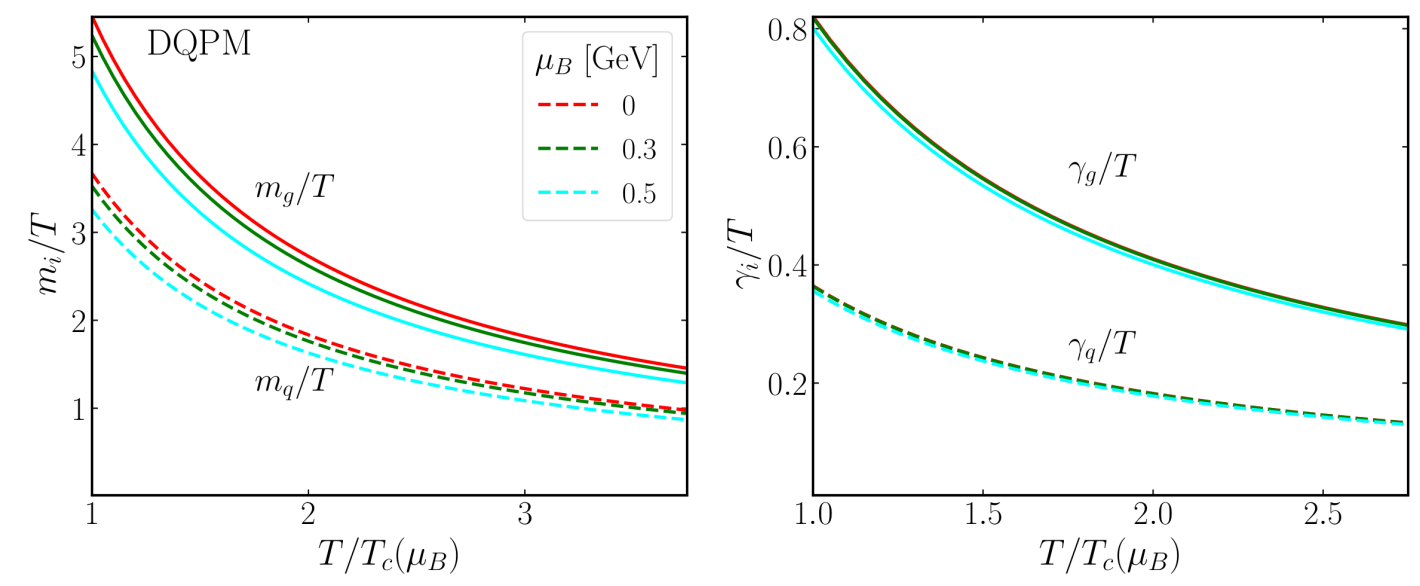

Figure 2.2: Ratios of the DQPM pole masses for light quarks (solid lines) and gluons (dashed lines) to temperature, $m_{i} / T$ (left), and ratios of the widths $\gamma_{i} / T$ (right), to temperature as a function of the scaled temperature $T / T_{c}\left(\mu_{B}\right)$ for fixed $\mu_{B}=0,0.3,0.5$ $\mathrm{GeV}$.

Fig. 2.1. At vanishing quark (baryon) chemical potential one can see a good agreement between the 1QCD evaluation of the QCD running coupling for $N_{f}=2$ (black triangles) [59] and the DQPM running coupling (red line). The DQPM running coupling $\alpha_{s}$ decreases with $\mu_{B}$ in the vicinity of the phase transition $T_{c} \leq T \leq 1.5 T_{c}$.

The pole masses of quasiparticles are chosen in the form of asymptotic quark or gluon masses respectively $m_{\infty} \sim m_{D} / 2$ or $\sqrt{2} m_{f}$, where $m_{D}$ is the HTL Debye mass, and $m_{f}$ is the HTL thermal fermion mass [61,62]:

$$
m_{g}^{2}\left(T, \mu_{\mathrm{B}}\right)=C_{g} \frac{g^{2}\left(T, \mu_{\mathrm{B}}\right)}{6} T^{2}\left(1+\frac{N_{f}}{2 N_{c}}+\frac{1}{2} \frac{\sum_{q} \mu_{q}^{2}}{T^{2} \pi^{2}}\right),
$$



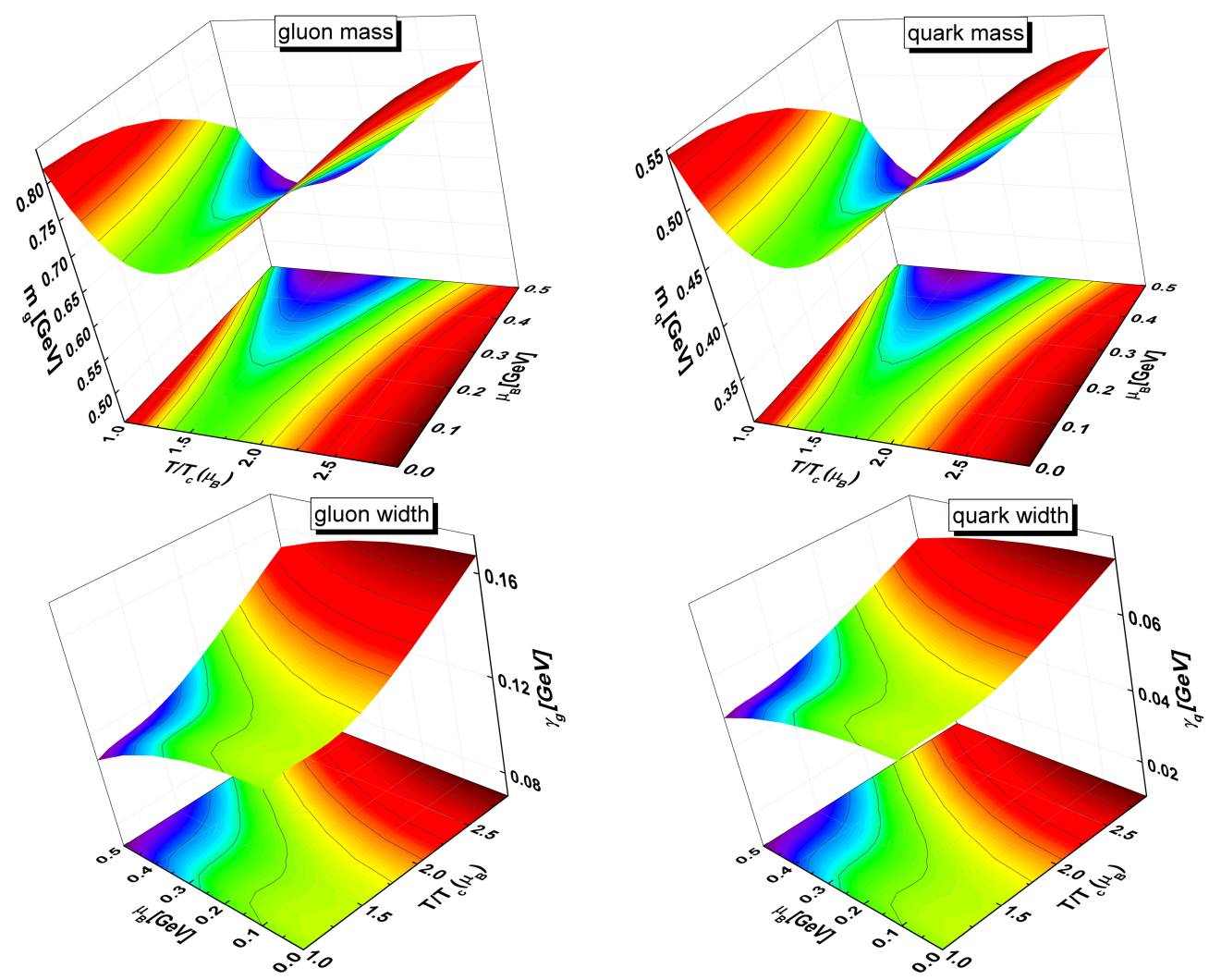

Figure 2.3: Gluon (left panel) and quark (right panel) pole-masses $m_{i}$ (upper row) and their widths $\gamma_{i}$ (lower row) from the DQPM as a function of the scaled temperature $T / T_{c}\left(\mu_{\mathrm{B}}\right)$ and baryon chemical potential $\mu_{\mathrm{B}}[4]$.

$$
m_{q(\bar{q})}^{2}\left(T, \mu_{\mathrm{B}}\right)=C_{q} \frac{g^{2}\left(T, \mu_{\mathrm{B}}\right)}{4} T^{2}\left(1+\frac{\mu_{q}^{2}}{T^{2} \pi^{2}}\right) .
$$

In Eqs. (32),(33) $N_{c}=3$ and $N_{f}=3$ denote the number of colors and the number of flavors respectively, $C_{q}=\frac{N_{c}^{2}-1}{2 N_{c}}=4 / 3$ and $C_{g}=N_{c}=3$ are the QCD color factors for quarks and for gluons, respectively. The strange quark has a larger bare mass which enhances its dynamical mass. This essentially suppresses the channel $g \rightarrow s+\bar{s}$ relative to the channel $g \rightarrow u+\bar{u}$ or $d+\bar{d}$ and controls the strangeness ratio in the QGP. Empirically $m_{s}\left(T, \mu_{B}\right)=$ $m_{u}\left(T, \mu_{B}\right)+\Delta m=m_{d}\left(T, \mu_{B}\right)+\Delta m$ where $\Delta m=30 \mathrm{MeV}$ has been used. This model parameter has been fixed in an empirical way by comparing to experimental data for strange hadron abundances and the $K^{+} / \pi^{+}$ratio from heavy-ion collisions at relativistic energies obtained within - the PHSD approach - a microscopic covariant transport approach. The detailed description of the transport approach will be given in the next Chapter. In the PHSD the deconfined phase evolution is based on the DQPM model - for the description of the QGP phase and involves a dynamical description of the hadronization process from partonic to hadronic matter (cf. the reviews [43,62]).

For the kinetic treatment it is important to consider finite widths of quasiparticles, which affect the transport properties of the QGP matter. The thermal widths have been estimated within the ressumation of hard thermal loops, which are results in the form $\gamma \sim g^{2} T \ln (1 / g)$ 
[63-65]. Therefore, thermal widths in the DQPM are adopted in a following form [47,62]:

$$
\gamma_{j}\left(T, \mu_{\mathrm{B}}\right)=\frac{1}{3} C_{j} \frac{g^{2}\left(T, \mu_{\mathrm{B}}\right) T}{8 \pi} \ln \left(\frac{2 c_{m}}{g^{2}\left(T, \mu_{\mathrm{B}}\right)}+1\right)
$$

Here, the parameter $c_{m}=14.4$ was fixed in $[1,45]$, which is related to a magnetic cut-off. Furthermore, we assume that all (anti-)quarks have the same thermal width: $\gamma_{u}=\gamma_{d}=\gamma_{s}$

Additionally, we consider scalar degrees of freedom and therefore gluons have only transverse polarisations. It was argued in Ref. [66] that the contribution of the longitudinal gluons to the general thermodynamic potential is subdominant compared to the contribution from the transverse gluons. We recall that for large momenta, $|\mathbf{p}|>>g T$, the longitudinal modes have an exponentially vanishing residue [67].

\subsection{Thermodynamic quantities in the DQPM for the crossover region}

In order to evaluate basic thermodynamic properties of QCD one can apply the $\Phi$-functional approach, where the thermodynamic potential $\Omega$ can be expressed in terms of dressed propagators $\Delta_{i}$. The derivatives of $\Omega$ : entropy density $s^{\mathrm{dqp}}=-\frac{\partial \Omega / V}{\partial T}$ and baryon density $n^{\text {dqp }}=-\frac{\partial \Omega / V}{\partial \mu}$, then can be easily found by the stationarity of $\Omega$ under functional variation with respect to the dressed propagators. Once the quasiparticle properties (or propagators) are fixed as described above, one can evaluate the entropy density $s\left(T, \mu_{\mathrm{B}}\right)$, the pressure $p\left(T, \mu_{\mathrm{B}}\right)$ and the energy density $\epsilon\left(T, \mu_{\mathrm{B}}\right)$ in a straight-forward manner by starting with the entropy density $s^{\text {dqp }}$ and the number density $n^{\text {dqp }}$ in the propagator representation from Baym $[68,69]$ and then identifying $s=s^{\mathrm{dqp}}$ and $n_{B}=n^{\mathrm{dqp}} / 3$ [2]:

$$
\begin{aligned}
s^{\mathrm{dqp}}\left(T, \mu_{q}\right)= & -d_{g} \int \frac{d^{4} P}{(2 \pi)^{4}} \frac{\partial f_{g}}{\partial T}\left(\operatorname{Im}\left(\ln \Delta^{-1}\right)-\operatorname{Im} \Pi \operatorname{Re} \Delta\right) \\
& -d_{q} \sum_{q=u, d, s} \int \frac{d^{4} P}{(2 \pi)^{4}} \frac{\partial f_{q}\left(\omega-\mu_{q}\right)}{\partial T}\left(\operatorname{Im}\left(\ln S_{q}^{-1}\right)-\operatorname{Im} \Sigma_{q} \operatorname{Re} S_{q}\right) \\
& -d_{\bar{q}} \sum_{\bar{q}=\bar{u}, \bar{d}, \bar{s}} \frac{d^{4} P}{(2 \pi)^{4}} \frac{\partial f_{\bar{q}}\left(\omega+\mu_{q}\right)}{\partial T}\left(\operatorname{Im}\left(\ln S_{\bar{q}}^{-1}\right)-\operatorname{Im} \Sigma_{\bar{q}} \operatorname{Re} S_{\bar{q}}\right), \\
n^{\mathrm{dqp}}\left(T, \mu_{q}\right)= & -\sum_{q=u, d, s} d_{q} \int \frac{d^{4} P}{(2 \pi)^{4}} \frac{\partial f_{q}\left(\omega-\mu_{q}\right)}{\partial \mu_{q}}\left(\operatorname{Im}\left(\ln S_{q}^{-1}\right)-\operatorname{Im} \Sigma_{q} \operatorname{Re} S_{q}\right) \\
& -\sum_{\bar{q}=\bar{u}, \bar{d}, \bar{s}} d_{\bar{q}} \int \frac{d^{4} P}{(2 \pi)^{4}} \frac{\partial f_{\bar{q}}\left(\omega+\mu_{q}\right)}{\partial \mu_{q}}\left(\operatorname{Im}\left(\ln S_{\bar{q}}^{-1}\right)-\operatorname{Im} \Sigma_{\bar{q}} \operatorname{Re} S_{\bar{q}}\right),
\end{aligned}
$$

where $\frac{d^{4} P}{(2 \pi)^{4}}=\frac{d \omega}{2 \pi} \frac{d^{3} \mathbf{p}}{(2 \pi)^{3}}, f_{g}(\omega)$ and $f_{q}\left(\omega-\mu_{q}\right)$ denote the Bose-Einstein and Fermi-Dirac distribution functions (see Eq. (58)), respectively, while $\Delta_{i}=\left(p^{2}-\Pi_{i}\right)^{-1}, S_{q}=\left(p^{2}-\Sigma_{q}\right)^{-1}$ and $S_{\bar{q}}=\left(p^{2}-\Sigma_{\bar{q}}\right)^{-1}$ stand for the full (scalar) quasiparticle propagator of gluons $g$, 
quarks $q$ and antiquarks $\bar{q}$. Here we consider for simplicity scalar (retarded) quasiparticle
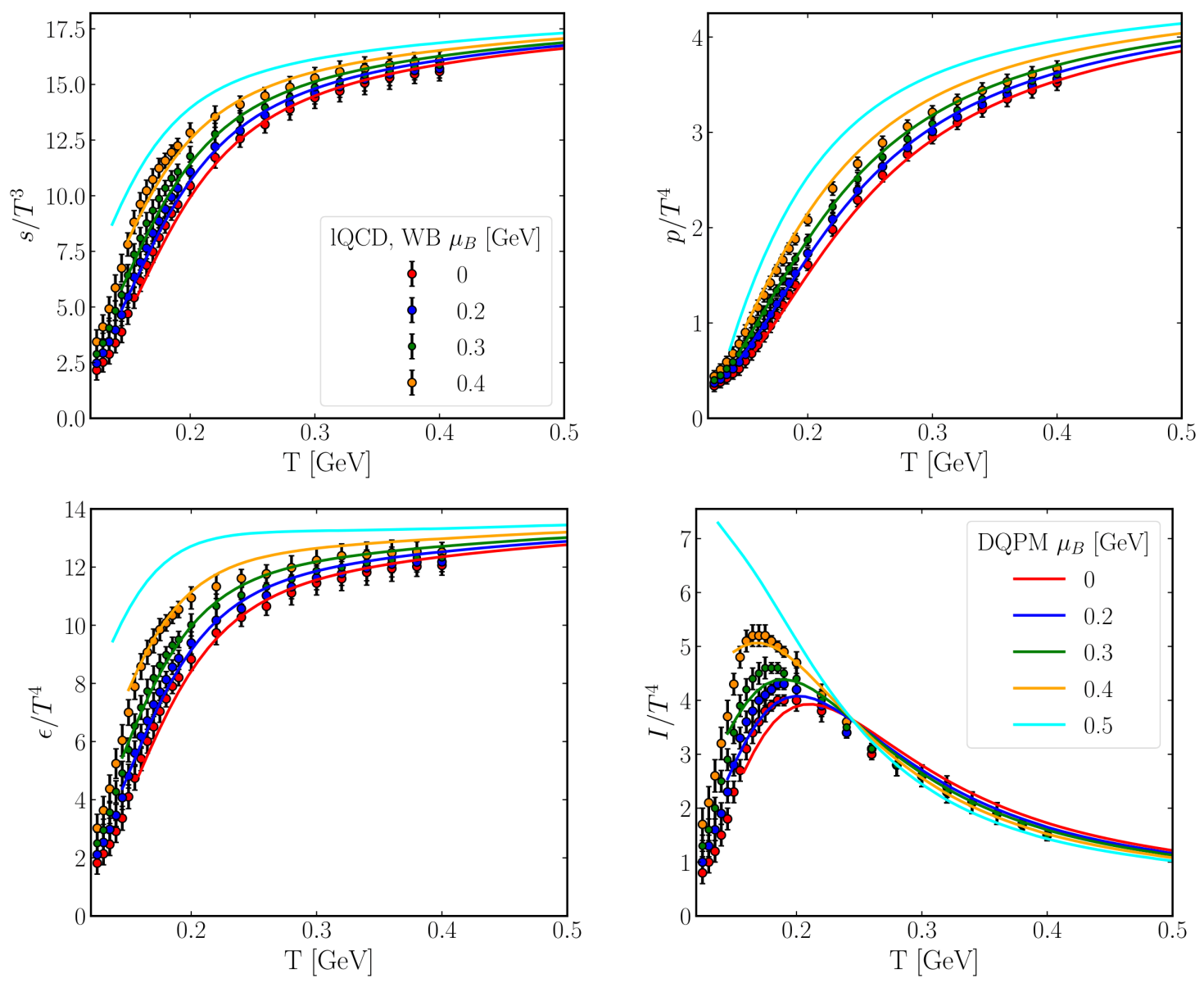

Figure 2.4: DQPM thermodynamic observables (solid lines): scaled entropy density $s / T^{3}$ (top left), pressure $p / T^{4}$ (top right), energy density $\epsilon / T^{4}$ (bottom left) and interaction measure $I / T^{4}$ (bottom right) as a function of temperature $T$ and at fixed baryon chemical potential $\mu_{B}$ ranging between 0 and $0.5 \mathrm{GeV}$. The $1 \mathrm{QCD}$ results obtained by the BMW collaboration are taken from Refs. $[22,60]$ (circles) for $\mu_{B}=0,0.2,0.3,0.4 \mathrm{GeV}$.

self-energies $\Pi_{i}=\Sigma_{i}=\Sigma_{q} \approx \Sigma_{\bar{q}}$, which are expressed via dynamical masses and widths as $\Pi_{i}=m_{i}^{2}-2 i \gamma_{i} \omega$, where, for the off-shell case, $\omega$ is an independent variable. Furthermore, the number of transverse gluonic degrees of freedom is $d_{g}=2 \times\left(N_{c}^{2}-1\right)$ while for the fermion degrees of freedom we use $d_{q}=2 \times N_{c}$ and $d_{\bar{q}}=2 \times N_{c}$.

The main contribution to the entropy density comes from light quarks and antiquarks, while strange quarks have smaller contributions due to their larger mass. The contribution of gluons is of the same order, although these have an even larger mass and width, due to the degeneracy factor $d_{g}=16$, which is larger than the strange quark degeneracy factor $d_{s}=6$.

The pressure of the system, $p\left(T, \mu_{\mathrm{B}}\right)$, which is considered isotropic, can be obtained by using the Maxwell relation of a grand-canonical ensemble:

$$
p\left(T, \mu_{\mathrm{B}}\right)=p_{0}(T, 0)+\int_{0}^{\mu_{\mathrm{B}}} n_{B}\left(T, \mu_{\mathrm{B}}^{\prime}\right) d \mu_{\mathrm{B}}^{\prime} .
$$


In Eq. (37), the pressure at vanishing baryon chemical potential $\mu_{B}=0$ is defined employing the entropy density as

$$
p_{0}(T, 0)=p^{l q c d}\left(T_{0}, 0\right)+\int_{T_{0}}^{T} s\left(T^{\prime}, 0\right) d T^{\prime},
$$

where the lower bound can be chosen between $0.12<T_{0}<0.15 \mathrm{GeV}$. We use here $T_{0}=$ $0.145 \mathrm{GeV} . p^{l q c d}\left(T_{0}, 0\right)$ is the 1QCD pressure taken from Refs. [22,60].

The energy density $\epsilon$ then follows from the Euler relation

$$
\epsilon=T s-p+\sum_{i} \mu_{i} n_{\mathrm{i}}
$$

where in general $i=B, Q, S$, while in this Chapter we consider only a symmetric setup for the quark chemical potentials $\mu_{q}=\mu_{u}=\mu_{s}=\mu_{B} / 3$ with vanishing strange and the electric charge potentials $\mu_{S}=\mu_{Q}=0(i=B)$. Another interesting thermodynamic observable, the trace of the energy-momentum tensor, is also known as interaction measure or the trace anomaly,

$$
I=\epsilon-3 p=T s-4 p+\sum_{i} \mu_{i} n_{\mathrm{i}}
$$

which has been found in the IQCD calculations [21,60,70,71] to be far from zero in the vicinity of the phase transition and indicates that the QGP matter in equilibrium can not be considered as a conformal fluid. Therefore, one can expect that the bulk viscosity of the QCD matter is finite near the phase transition.

To wind up, the model parameters are fixed at $\mu_{B}=0$, with the 1QCD entropy density and pressure as input parameters for the calculations, and at finite $\mu_{B}$ the 'scaling hypothesis' enables the DQPM to make predictions for thermodynamic and transport properties. Figure 2.4 demonstrates the dimensionless thermodynamic observables from the DQPM (solid lines) in comparison to the $1 \mathrm{QCD}$ data from the BMW collaboration [22,60] (circles) for fixed baryon chemical potentials. We find good agreement between the DQPM results and the lattice points within the error bars.

We now proceed with the second derivatives of the thermodynamic potential $\Omega=-p$ : the speed of sound squared $c_{s}^{2}=\frac{d p}{d \epsilon}$ and specific heat at constant volume $C_{V}=\frac{d \epsilon}{d T}$. At fixed $\mu_{B}$ the speed of sound can be expressed via the specific heat as:

$$
c_{s}^{2}=\frac{d p}{d \epsilon}=\frac{d p / d T}{d \epsilon / d T}=\frac{s}{C_{V}}
$$

The resulting speed of sound squared $c_{s}^{2}$ and the dimensionless specific heat at constant volume $C_{V} / T^{3}$ are depicted in Fig. 2.5 as a function of the scaled temperature $T / T_{c}\left(\mu_{\mathrm{B}}\right)$ and baryon chemical potential $\mu_{\mathrm{B}}$. The $1 \mathrm{QCD}$ data are shown at vanishing chemical potential: results from the Wuppertal-Budapest collaboration [22] (black spheres) and the HotQCD collaboration [72] (blue spheres). The DQPM estimates of $c_{s}^{2}$ agree well with the Wuppertal-Budapest results for the speed of sound squared, while the HotQCD results are smaller in the region of $T \geq 1.5 T_{c}$. Therefore, taking into account relations between $c_{s}^{2}$ 

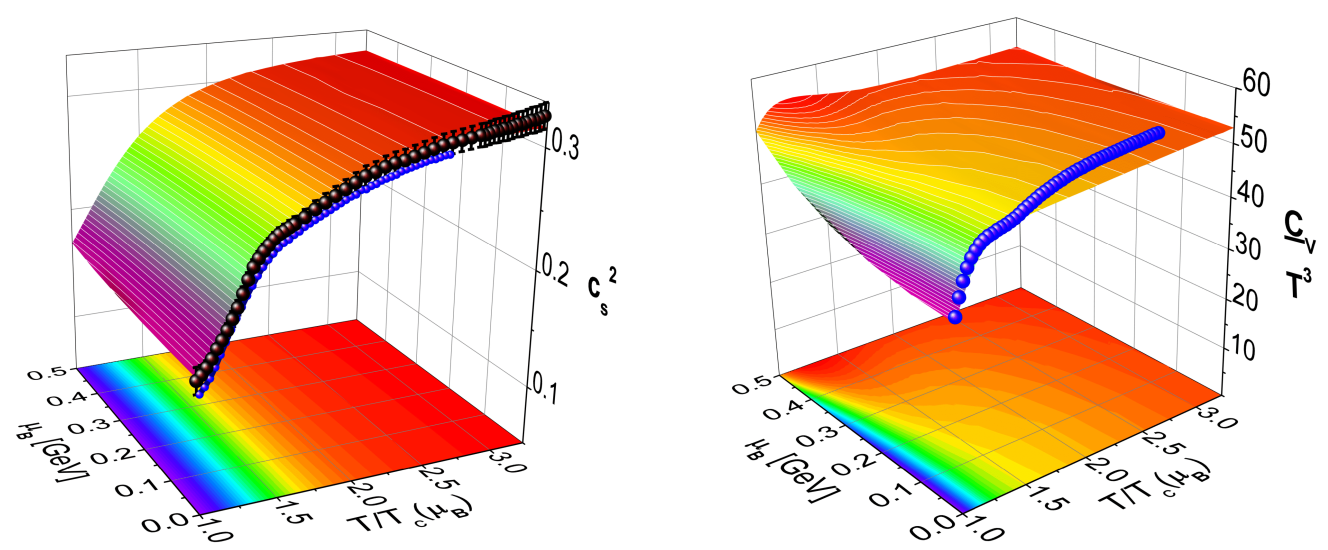

Figure 2.5: speed of sound squared $c_{s}^{2}$ (left) and scaled specific heat $C_{V} / T^{3}$ (right) from the DQPM as a function of the scaled temperature $T / T_{c}\left(\mu_{\mathrm{B}}\right)$ and baryon chemical potential $\mu_{\mathrm{B}}$. At vanishing chemical potential we compare to $1 \mathrm{QCD}$ results obtained by the Wuppertal-Budapest collaboration [22] (black spheres) and the HotQCD collaboration [72] (blue spheres).

and $C_{V} / T^{3}$, it is expected that the HotQCD results for the dimensionless ratio $C_{V} / T^{3}$ are slightly higher than the DQPM estimates for $T \geq 1.5 T_{c}$.

\subsection{Partonic interactions: cross sections and interaction rates}

The key ingredients of the transport equations are the elastic cross sections of the relevant degrees of freedom. A distinctive feature of the DQPM is that quarks and gluons have nonvanishing thermal masses that depend on $\left(T, \mu_{B}\right)$ as well as the effective coupling $g^{2}\left(T, \mu_{B}\right)$ that enters the scattering amplitudes. The differential cross section for a binary scattering of on-shell particles $(i+j \rightarrow c+d)$ in the center of mass frame (CM), where the momenta of the colliding particles obey $\mathbf{k}_{i}+\mathbf{k}_{j}^{\prime}=\mathbf{p}_{c}+\mathbf{p}_{d}^{\prime}=0$ and $k_{i}^{0}+{k^{\prime}}_{j}^{0}=\sqrt{s}=p_{c}^{0}+{p^{\prime}}_{d}^{0}$, is given by

$$
\mathrm{d} \sigma_{i j \rightarrow c d}^{o n}(\sqrt{s}, \Omega)=\frac{p_{f} d \Omega}{16 \pi^{2} \sqrt{s}} \frac{|\overline{\mathcal{M}}|^{2}}{F^{\mathrm{CM}}}=\frac{1}{64 \pi^{2} s} \frac{p_{\text {out }}}{p_{\text {in }}}|\overline{\mathcal{M}}|^{2} \mathrm{~d} \Omega
$$

with $F^{\mathrm{CM}}=4 p_{i} \sqrt{s}$ being the flux factor in the CM frame, $d \Omega$ - the differential solid angle corresponding to one of the final particles, while $|\overline{\mathcal{M}}|^{2} \equiv\left|\overline{\mathcal{M}}\left(k_{i_{1}} \ldots k_{i_{n}} \rightarrow p_{j_{1}} \ldots p_{j_{m}}\right)\right|^{2}$ denotes the invariant matrix element squared, averaged over the color and spin of the incoming particles and summed over those of the final particles. The total cross section is obtained by

$$
\sigma_{\text {tot }}^{i j \rightarrow c d}(\sqrt{s})=\frac{1}{32 \pi s} \frac{p_{\text {out }}}{p_{\text {in }}} \gamma_{i j} \int_{-1}^{1} d \cos (\theta)|\overline{\mathcal{M}}|^{2},
$$

where $\theta$ is the final polar angle of one of the final particles in the CM frame, and $\gamma_{i j}=$ $1-\frac{1}{2} \delta_{i j}$ is the symmetry factor. In this frame, the collision is independent from the azimuthal angle $\phi$ and the corresponding integration boils down to a constant multiplier of $2 \pi$. 

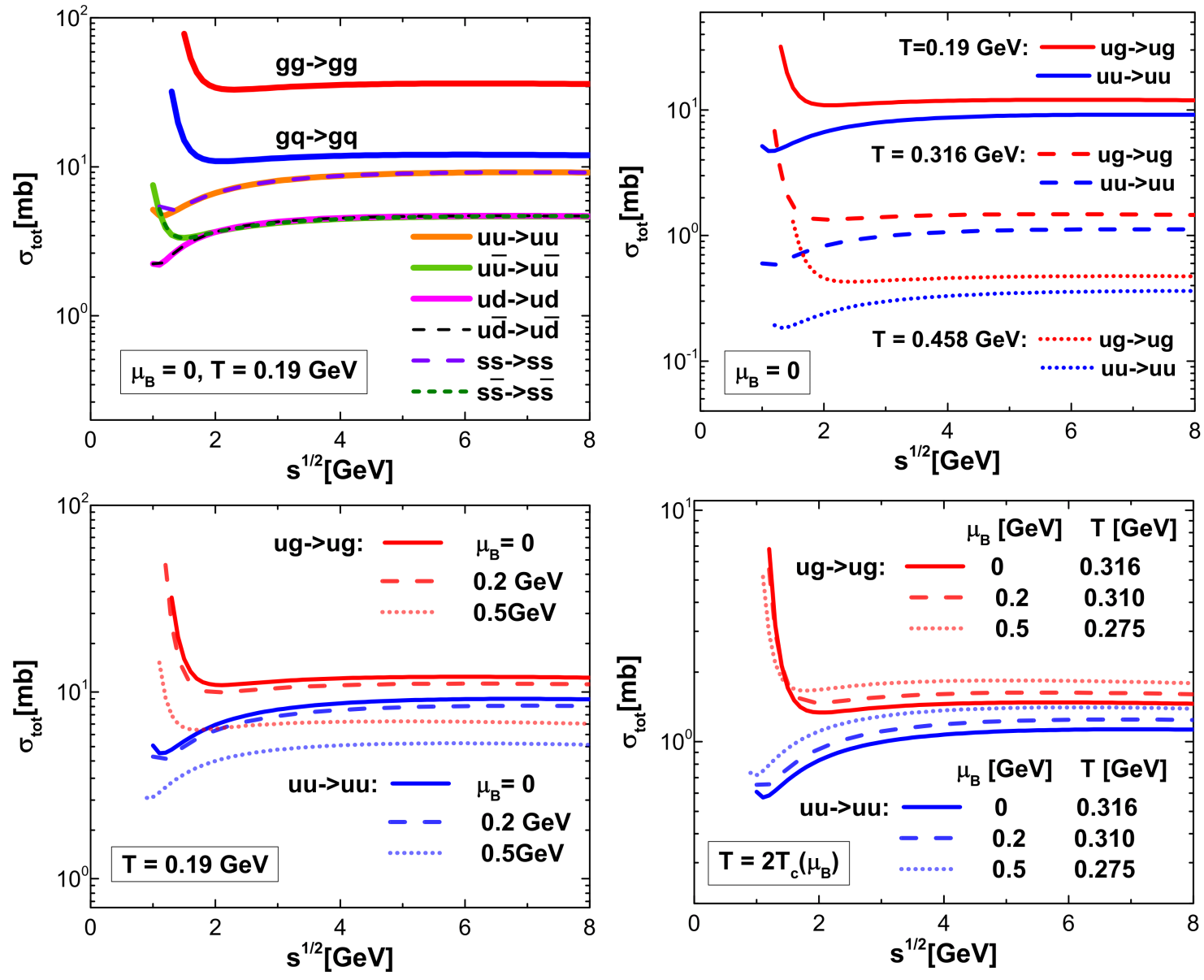

Figure 2.6: DQPM total elastic cross sections between different partons for the on-shell case from Eq. ( 43) evaluated in the center of mass of the collision system as a function of the collision energy $\sqrt{s}$ for $\mu_{B}=0, T=0.19 \mathrm{GeV}$ (top left), $\mu_{B}=0, T=0.19,0.316,0.458$ $\mathrm{GeV}$ (top right), $\mu_{B}=0,0.2,0.5 \mathrm{GeV}$ (bottom left) and for various values of baryon chemical potential $\mu_{\mathrm{B}}=0,0.2,0.5 \mathrm{GeV}$ for $T=2 T c\left(\mu_{\mathrm{B}}\right)$ (bottom right). The initial masses of the colliding partons are taken as the pole masses. The figure is taken from [6]. 
Momenta of the initial $(i)$ and final particles $(f)$ in the CM frame are found to be

$$
p_{i, f}=\frac{\sqrt{\left(s-\left(m_{i, f}+m_{i, f}^{\prime}\right)^{2}\right)\left(s-\left(m_{i, f}-m_{i, f}^{\prime}\right)^{2}\right)}}{2 \sqrt{s}}
$$

with $m_{i, f}$ and $m_{i, f}^{\prime}$ being the masses of the colliding partons.

The DQPM total cross sections for different channels are shown in Fig. 2.6 as a function of collision energy $\sqrt{s}$ for (a) $\mu_{B}=0, T=0.19 \mathrm{GeV}$, (b) $\mu_{B}=0, T=0.19,0.316,0.458 \mathrm{GeV}$, (c) $\mu_{B}=0,0.2,0.5 \mathrm{GeV}$ and (d) for various values of baryon chemical potential $\mu_{\mathrm{B}}=0,0.2$, $0.5 \mathrm{GeV}$ for $T=2 T c\left(\mu_{\mathrm{B}}\right)(T=0.316,0.310,0.275 \mathrm{GeV})$. The initial masses of the colliding partons are taken as pole masses from Eqs. (33) - (32) to give the same initial flux $F$. For all temperatures $T$ the cross section does not change much with the collision energy $\sqrt{s}$. Total cross sections at fixed $\sqrt{s}$ depend on temperature as $\sigma_{\text {tot }}^{i j \rightarrow c d} \sim 1 / T^{3}$ or $\sim 1 / T^{4}$, which is governed by the $T$-dependence of the DQPM running coupling demonstrated in Fig. 28. The difference in the $T$-dependence for different channels arise from the combinations of $s-, t-, u-$ channels: for $q-q, q-\bar{q}$ and $q-g$ scatterings $\sigma_{\text {tot }}^{i j \rightarrow c d} \sim 1 / T^{3}$, while for the $g-g$ channel the terms $1 / T^{3}, 1 / T^{4}$ have equivalent contributions to the total cross section $\sigma_{\text {tot }}^{i j \rightarrow c d} \sim c_{3} / T^{3}+c_{4} / T^{4}$, where $c_{3}, c_{4}$ depend on $\sqrt{s}, \mu_{B}$ [1]. The cross sections decrease with increasing baryon chemical potential as expected from the $\mu_{B}$-dependence of the DQPM coupling. This trend is in agreement with the pQCD predictions for high temperatures and chemical potential, where the QGP can be described approximately by a free gas of quarks and gluons.

Further, we consider the "off-shell" scattering, where the energy of partons and their moments are independent degrees of freedom, and a general definition of the "off-shell cross section" is not applicable due to the absence of asymptotically stable states. Nevertheless, the transition matrix elements for various incoming and outgoing 4-momenta can be well defined off-shell. To include the off-shell effects to the scattering of time-like particles one can the Lorentz invariant phase space in Eq. (42), in the case of a incoming flux $F=v_{\text {rel }} 2 \omega_{i} 2 \omega_{j}$ - integrating over the energy of the final time-like particles as

$$
\begin{aligned}
d \sigma^{\mathrm{off}}= & \frac{1}{F} \frac{d^{4} p_{c}}{(2 \pi)^{4}} \frac{d^{4} p_{d}}{(2 \pi)^{4}} \tilde{\rho}_{c}\left(\omega_{c}, \mathbf{p}_{c}\right) \theta\left(\omega_{c}\right) \tilde{\rho}_{d}\left(\omega_{d}, \mathbf{p}_{d}\right) \theta\left(\omega_{d}\right) \\
& \times(2 \pi)^{4} \delta^{(4)}\left(p_{i}+p_{j}-p_{c}-p_{d}\right)|\overline{\mathcal{M}}|^{2}
\end{aligned}
$$

In Eq. (45) we employed the renormalized time-like spectral functions

$$
\tilde{\rho}_{j}\left(\omega_{j}, \mathbf{p}_{j}\right)=\frac{\rho_{j}\left(\omega_{j}, \mathbf{p}_{j}\right) \theta\left(p_{j}^{2}\right)}{\int_{0}^{\infty} \frac{d \omega_{j}}{(2 \pi)} 2 \omega_{j} \rho_{j}\left(\omega_{j}, \mathbf{p}_{j}\right) \theta\left(p_{j}^{2}\right)} .
$$

Here the spectral function $\rho_{i}$ corresponding to the parton specie $i$ is taken from Eq. (25). The final parton masses are defined as $m_{i}^{2}=p_{i}^{2}=\omega_{i}^{2}-\mathbf{p}_{i}^{2}$. In the limit of vanishing 
thermal widths $\lim _{\gamma_{i} \rightarrow 0} \rho_{i}(\omega, \mathbf{p})=2 \pi \delta\left(\omega^{2}-\mathbf{p}^{2}-M_{i}^{2}\right)$ the off-shell cross section coinsides with the on-shell one as defined previously in Eq. (42).

Here we evaluate the differential 'off-shell cross section' for time-like quanta in the center of mass system of the collision for convenience. Using a $\delta$-function in Eq. (45), one can integrate over the four-momenta of particle $d$ to obtain the total cross section in the CM frame:

$$
\sigma_{\mathrm{off}}^{\mathrm{CM}}=\frac{1}{(2 \pi)^{3} F^{\mathrm{CM}}} \int_{0}^{\sqrt{s} / 2} p_{f}^{2} d p_{f} d \cos (\theta) \int_{p_{f}}^{\sqrt{s}-p_{f}} d \omega_{c}^{\mathrm{CM}} \tilde{\rho}_{c}\left(\omega_{c}, \mathbf{p}_{c}\right) \tilde{\rho}_{d}\left(\omega_{d}, \mathbf{p}_{d}\right)|\overline{\mathcal{M}}|^{2}
$$

with $F^{C M}=4 p_{i}^{\mathrm{CM}} \sqrt{s}$. It is important to notice that in off-shell case, the calculation of the cross section is performed in the center of mass system, nevertheless, the energies and momenta entering the spectral functions (25) should be expressed in the heat bath frame by applying the appropriate Lorentz transformations.

To continue with the calculations of the transport properties, let us briefly recall that an on-shell interaction rate, also known as a collisional rate, in the medium rest system, where the incoming quark has a four-momentum $P_{i}=\left(E_{i}, \mathbf{p}_{i}\right)$ (energies of the particles are taken to be $E_{i}^{2}=\mathbf{p}^{2}+m_{i}^{2}$ where $m_{i}$ is the pole mass) is given by:

$$
\begin{aligned}
& \Gamma_{i}^{\mathrm{on}}\left(p_{i}, T, \mu_{q}\right)=\frac{1}{2 E_{i}} \sum_{j=q, \bar{q}} \frac{1}{1+\delta_{c d}} \int \frac{\mathrm{d}^{3} p_{j}}{(2 \pi)^{3} 2 E_{j}} d_{q} f_{j}^{(0)} \\
& \times \int \frac{\mathrm{d}^{3} p_{c}}{(2 \pi)^{3} 2 E_{c}} \int \frac{\mathrm{d}^{3} p_{d}}{(2 \pi)^{3} 2 E_{d}}|\overline{\mathcal{M}}|^{2}\left(p_{i}, p_{j}, p_{c}, p_{d}\right) \\
& \times(2 \pi)^{4} \delta^{(4)}\left(p_{i}+p_{j}-p_{c}-p_{d}\right)\left(1-f_{c}^{(0)}\right)\left(1-f_{d}^{(0)}\right) \\
& =\frac{1}{2 E_{i}} \sum_{j=q, \bar{q}} \frac{1}{1+\delta_{c d}} \int \frac{\mathrm{d}^{3} p_{j}}{(2 \pi)^{3} 2 E_{j}} d_{q} f_{j}^{(0)} \\
& \times \frac{1}{16 \pi \sqrt{s}} \frac{1}{p_{c m}} \int \mathrm{d} t|\overline{\mathcal{M}}|^{2}(s, t)\left(1-f_{c}^{(0)}\right)\left(1-f_{d}^{(0)}\right) \\
& =\sum_{j=q, \bar{q}} \int \frac{\mathrm{d}^{3} p_{j}}{(2 \pi)^{3}} d_{q} f_{j}^{(0)} v_{\mathrm{rel}} \sigma_{i j \rightarrow c d}\left(s, T, \mu_{q}\right),
\end{aligned}
$$

where $d_{j}$ is the degeneracy factor for spin and color (for quarks $d_{q}=2 \times N_{c}$ and for gluons $d_{g}=2 \times\left(N_{c}^{2}-1\right)$ ), and with the shorthand notation $f_{j}=f_{j}\left(E_{j}, T, \mu_{q}\right)$ for the distribution functions. In Eq. (151) and in all this Section, the notation $\sum_{j=q, \bar{q}, g}$ includes the contribution from all possible partons which in our case are gluons and (anti-) quarks of three different flavors $(u, d, s)$.

Furthermore, the relative velocity $v_{\text {rel }}$ of the colliding partons in the c.m. frame, is given by

$$
v_{\text {rel }}=\frac{\sqrt{\left(p_{i} \cdot p_{j}\right)^{2}-m_{i}^{2} m_{j}^{2}}}{E_{i} E_{j}}=\frac{p_{c m} \sqrt{s}}{E_{i} E_{j}} .
$$


$p_{c m}$ denotes the momenta of the initial $(i, j)$ as well as of the final quarks $(c, d)$ in the CM frame given by Eq. (44).

Furthermore, one can generalize interaction rate for the off-shell case. In order to obtain the interaction rate of the off-shell DQPM time-like partons, we have to calculate the interaction rate for the corresponding parton $i$ with momentum $\mathbf{p}_{i}$ due to collisions with time-like particles $j$ leading to final time-like particles $c$ and $d$ by integrating additionally over all energies $\omega_{j}$ in the time-like sector [1]:

$$
\begin{aligned}
& \Gamma_{i}^{\mathrm{off}}\left(\mathbf{p}_{i}, T, \mu_{q}\right)=\int_{0}^{\infty} \frac{d \omega_{i}}{(2 \pi)} \tilde{\rho}_{i} \sum_{j=q, \bar{q}, g} \frac{1}{1+\delta_{c d}} \int \frac{d^{4} p_{j}}{(2 \pi)^{4}} \theta\left(\omega_{j}\right) d_{j} \tilde{\rho}_{j} f_{j}^{(0)} \\
& \times \int \frac{d^{4} p_{c}}{(2 \pi)^{4}} \theta\left(\omega_{c}\right) \tilde{\rho}_{c} \int \frac{d^{4} p_{d}}{(2 \pi)^{4}} \theta\left(\omega_{d}\right) \tilde{\rho}_{d}\left(1 \pm f_{c}^{(0)}\right)\left(1 \pm f_{d}^{(0)}\right) \\
& \quad \times|\overline{\mathcal{M}}|^{2}\left(p_{i}, p_{j}, p_{c}, p_{d}\right)(2 \pi)^{4} \delta^{(4)}\left(p_{i}+p_{j}-p_{c}-p_{d}\right) .
\end{aligned}
$$

Here the shorthand notation $\tilde{\rho}$ (46) for the renormalized time-like spectral functions $\tilde{\rho}_{j}\left(\omega_{j}, \mathbf{p}_{j}\right)$ has been used and $f_{j}=f_{j}\left(\omega_{j}, T, \mu_{q}\right)$ for the distribution functions. We mention that interaction rate of time-like off-shell partons by Eq. (50) discards damping processes between the time-like and space-like sectors, which are assumed to be subleading. To evaluate the average time-like interaction rate of partons $i$, we finally have to average its interaction rate as

$$
\begin{aligned}
& \Gamma_{i}^{\text {off }}\left(T, \mu_{q}\right)=\frac{d_{i}}{n_{i}^{\text {off }}\left(T, \mu_{q}\right)} \int \frac{d^{4} p_{i}}{(2 \pi)^{4}} \theta\left(\omega_{i}\right) \tilde{\rho}_{i} f_{i}^{(0)} \\
& \quad \times \sum_{j=q, \bar{q}, g} \frac{1}{1+\delta_{c d}} \int \frac{d^{4} p_{j}}{(2 \pi)^{4}} \theta\left(\omega_{j}\right) d_{j} \tilde{\rho}_{j} f_{j}^{(0)} \int \frac{d^{4} p_{c}}{(2 \pi)^{4}} \theta\left(\omega_{c}\right) \tilde{\rho}_{c} \int \frac{d^{4} p_{d}}{(2 \pi)^{4}} \theta\left(\omega_{d}\right) \tilde{\rho}_{d} \\
& \quad \times|\overline{\mathcal{M}}|^{2}\left(p_{i}, p_{j}, p_{c}, p_{d}\right)(2 \pi)^{4} \delta^{(4)}\left(p_{i}+p_{j}-p_{c}-p_{d}\right)\left(1 \pm f_{c}^{(0)}\right)\left(1 \pm f_{d}^{(0)}\right),
\end{aligned}
$$

with the off-shell density of time-like partons $i$ given by

$$
n_{i}^{\mathrm{off}}\left(T, \mu_{q}\right)=d_{i} \int \frac{d^{4} p_{i}}{(2 \pi)^{4}} \theta\left(\omega_{i}\right) 2 \omega_{i} \tilde{\rho}_{i} f_{i}^{(0)}\left(\omega_{i}, T, \mu_{q}\right)
$$

In fact, one can estimate the asymptotic temperature behaviour of the parton interaction rate taking into account that the interaction rate of parton $i$ is directly proportional to the density of the colliding partner $j$ and its degeneracy factor $d_{j}$, and to their interaction cross section $\sigma_{i j}$ as:

$$
\Gamma_{i} \propto \sum_{j} d_{j} f_{j}^{(0)} \sigma_{i j}
$$

Let's first consider the interaction rate of the most abundant type of quark - light quark. 

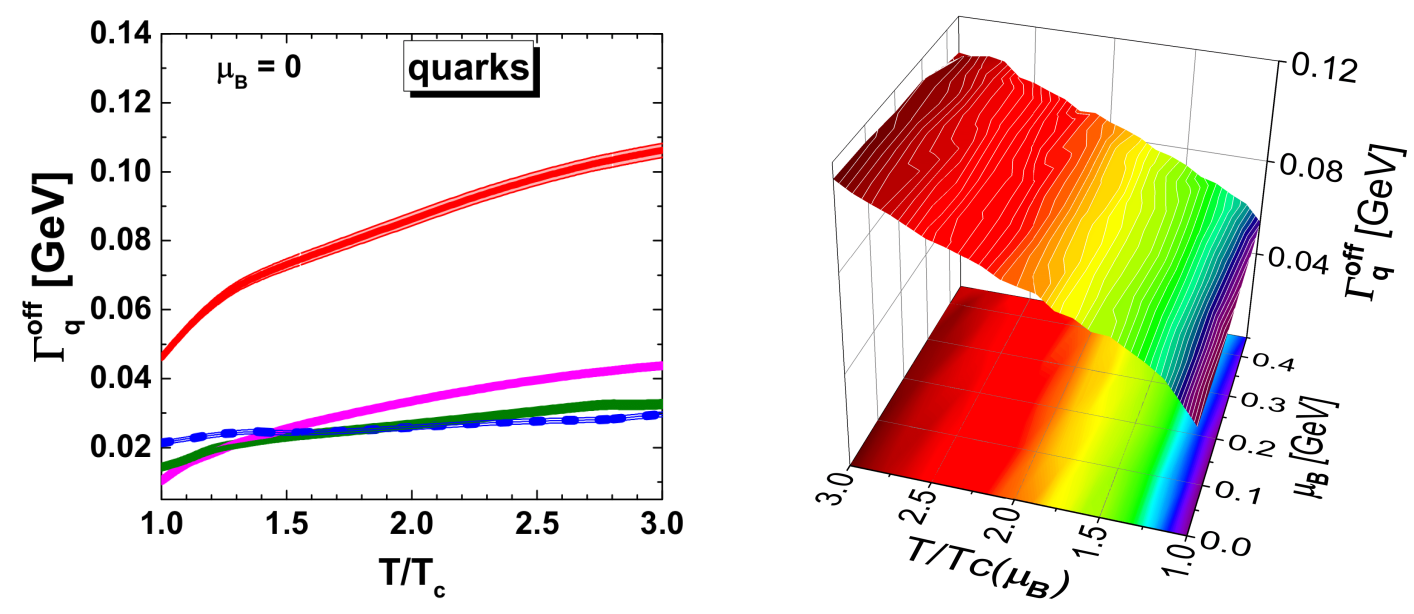

Figure 2.7: Quark off-shell interaction rate (left) as a function of scaled temperature $T / T_{c}$ for $\mu_{B}=0$ and (right) for moderate chemical potentials $\mu_{B} \leq 0.5 \mathrm{GeV}$ as a function of the scaled temperature $T / T_{c}\left(\mu_{B}\right)$ and the baryon chemical potential $\mu_{B}$. The contributions from the scattering with light quarks (green), antiquarks (blue), and gluons (pink) are given by the lower hatched bands, which arise from the finite statistics in the evaluation of the integrals by Monte Carlo.

Adding up all possible contributions we get:

$$
\begin{aligned}
\Gamma_{u} & =\sum_{q} \Gamma_{u q}+\sum_{\bar{q}} \Gamma_{u \bar{q}}+\Gamma_{u g} \\
& =\Gamma_{u u \rightarrow u u}+\Gamma_{u d \rightarrow u d}+\Gamma_{u s \rightarrow u s}+\Gamma_{u \bar{u} \rightarrow u \bar{u}}+\Gamma_{u \bar{u} \rightarrow d \bar{d}} \\
& +\Gamma_{u \bar{u} \rightarrow s \bar{s}}+\Gamma_{u \bar{d} \rightarrow u \bar{d}}+\Gamma_{u \bar{s} \rightarrow u \bar{s}}+\Gamma_{u g \rightarrow u g} .
\end{aligned}
$$

While the dependences on temperature are similar for fixed $\mu_{B}$, we see a general slight decrease in the total widths with $\mu_{B}$ for fixed temperatures. At vanishing quark chemical potential, the total interaction rate $\Gamma_{u}$ comes largely from quark-gluon scattering and increases with temperature while the contributions from scatterings with quarks and antiquarks are about equal and subdominant. At finite $\mu_{q}$, quarks are more abundant than antiquarks and contributions from scatterings with quarks increase, while contributions from collisions with antiquarks decrease relative to $\mu_{q}=\mu_{B}=0$. The contributions from collisions with gluons also decrease slightly with $\mu_{B}$, which is due to a decrease in the effective cross sections with $\mu_{B}$, as mentioned above.

Similarly for a gluon $g$, the total interaction rate is given by:

$$
\begin{aligned}
\Gamma_{g} & =\sum_{q} \Gamma_{g q}+\sum_{\bar{q}} \Gamma_{g \bar{q}}+\Gamma_{g g} \\
& =2 \times \Gamma_{g u \rightarrow g u}+\Gamma_{g s \rightarrow g s}+2 \times \Gamma_{g \bar{u} \rightarrow g \bar{u}}+\Gamma_{g \bar{s} \rightarrow g \bar{s}}+\Gamma_{g g \rightarrow g g} .
\end{aligned}
$$

Figure 2.7 shows the off-shell interaction rate for light quark as a function of the scaled 
temperature $T / T_{c}\left(\mu_{B}\right)$ and chemical potential $\mu_{B}$. While the dependencies on temperature are similar for fixed $\mu_{B}$, we see a general slight decrease of the total widths with $\mu_{B}$ for fixed $T / T_{c}\left(\mu_{B}\right)$ as discussed above.

\subsection{Transport coefficients of the QGP at finite $\mathrm{T}$ and $\mu_{B}$}

Herein we employ the relativistic kinetic theory in order to determine the transport coefficients with help of the relaxation time approximation of the Boltzmann equation for the quasiparticles with dynamical masses $M_{i}\left(T, \mu_{q}\right)$ [73-76]:

$$
k_{i}^{\mu} \partial_{\mu} f_{i}+\frac{1}{2} \partial^{\mu} M_{i}^{2} \partial_{\left(k_{i, \mu}\right)} f_{i}=\sum_{j=1}^{N_{\text {species }}} C_{i j}(x, k),
$$

where $C_{i j}(x, k)$ is the 2-body collision term which contains only quasi-elastic $2 \leftrightarrow 2$ scatterings, while the second term contains $F_{i}^{\mu}=\partial^{\mu} M_{i}$ and is an external force attributed to the residual mean field interaction due to the medium-dependent effective masses $M_{i}\left(T, \mu_{q}\right)$.

In order to evaluate transport coefficients we consider a small departure from equilibrium, where the distribution function can be expressed as

$$
f_{i}(x, k, t)=f_{i}^{(0)}(x, k, t)+f_{i}^{(1)}(x, k, t)=f_{i}^{(0)}(x, k, t)\left(1+\delta f_{i}(x, k, t)\right) .
$$

$f_{i}^{(0)}(x, k, t)$ is the local equilibrium distribution function, $f_{i}^{(1)}(x, k, t)$ contains $\delta f_{i}(x, k, t)$, which is the non-equilibrium part to first order in gradients. The equilibrium state of the system is described by the Bose-Einstein and Fermi-Dirac distribution functions

$$
f_{i}^{(0)}\left(E_{i}, T, \mu_{i}\right)=\frac{1}{\exp \left(\left(E_{i}-\mu_{i}\right) / T\right)-a_{i}}
$$

where $\mu_{i}$ is the quark chemical potential, $E_{i}=\sqrt{\mathbf{p}_{i}^{2}+m_{i}^{2}}$ is the on-shell quark/gluon energy, $a_{i} \equiv+1$ (gluons), -1 ((anti-)quarks). The on-shell parton density of the specie ${ }^{\prime} i$ is defined as

$$
n_{i}\left(T, \mu_{q}\right)=d_{i} \int \frac{d^{3} p}{(2 \pi)^{3}} f_{i}^{(0)}\left(E_{i}, T, \mu_{q}\right)
$$

where $i=u, d, s, \bar{u}, \bar{d}, \bar{s}, g$ and $d_{i}$ is the degeneracy factor for the corresponding parton.

\subsubsection{Parton relaxation times}

The first step in the calculation of transport coefficients within the RTA framework is the evaluation of relaxation times, which are supposed to depend on momenta, temperature, and baryon chemical potential. In the energy-dependent relaxation-time approximation, we assume particle species $i$ is out-of-equilibrium, while all other particle species are in 


\begin{tabular}{|c|c|c|c|c|}
\hline Parton & $\begin{array}{c}\text { Degeneracy } \\
d_{i}\end{array}$ & $\begin{array}{c}\text { Baryon Number } \\
b_{i}\end{array}$ & $\begin{array}{c}\text { Electric Charge } \\
q_{i} \cdot e\end{array}$ & $\begin{array}{c}\text { Strangeness } \\
s_{i}\end{array}$ \\
\hline$g$ & 16 & 0 & 0 & 0 \\
$u$ & 6 & $+1 / 3$ & $+2 / 3 e$ & 0 \\
$\bar{u}$ & 6 & $-1 / 3$ & $-2 / 3 e$ & 0 \\
$d$ & 6 & $+1 / 3$ & $-1 / 3 e$ & 0 \\
$\bar{d}$ & 6 & $-1 / 3$ & $+1 / 3 e$ & 0 \\
$s$ & 6 & $+1 / 3$ & $-1 / 3 e$ & -1 \\
$\bar{s}$ & 6 & $-1 / 3$ & $+1 / 3 e$ & +1 \\
\hline
\end{tabular}

Table 1: Fundamental properties of the parton species considered in the quark-gluon plasma. Here, $e=\sqrt{4 \pi \alpha_{e m}}$ denotes the elementary electric charge in natural units, while $q_{i}$ is dimensionless electric charge of the parton specie ' $i{ }^{\prime}$.
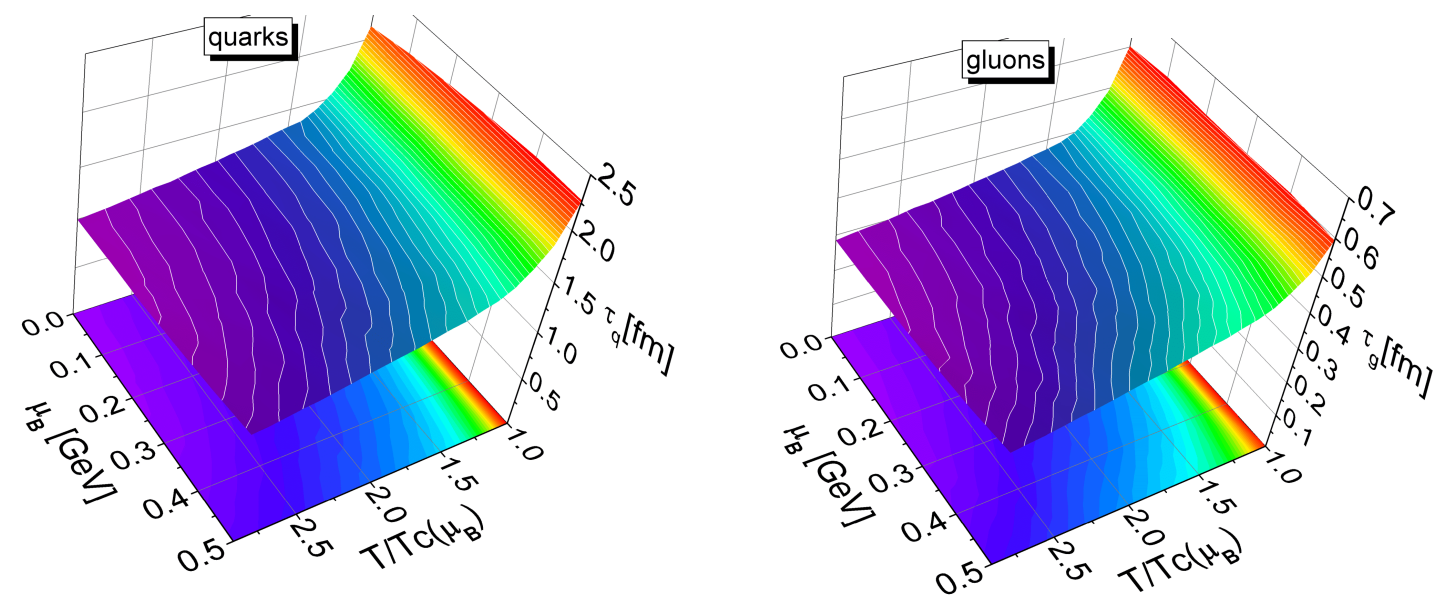

Figure 2.8: Relaxation time of a quark (left) and gluon (right) as a function of the scaled temperature $T / T_{c}\left(\mu_{B}\right)$ and the baryon chemical potential $\mu_{B}$ evaluated by the average parton interaction rate from Eq. (62).

equilibrium. In the general case the momentum-dependent relaxation time $\tau_{i}\left(\mathbf{p}, T, \mu_{B}\right)$ can be expressed through the on-shell interaction rate in the medium rest system where the incoming quark has a four momentum $P_{i}=\left(E_{i}, \mathbf{p}_{i}\right)[74,76-78]$ :

$$
\tau_{i}\left(\mathbf{p}, T, \mu_{B}\right)=\frac{1}{\Gamma_{i}\left(\mathbf{p}, T, \mu_{B}\right)}
$$

For comparison we can estimate the parton relaxation time by the spectral widths:

$$
\tilde{\tau}_{i}\left(T, \mu_{B}\right)=\frac{1}{2 \gamma_{i}\left(T, \mu_{B}\right)} .
$$

To quantify the temperature dependence of transport coefficients we have evaluated the average parton relaxation times as a function of temperature $T$ and chemical potential $\mu_{B}$. To this aim we calculate the average width of the partons $i$, and average its interaction 
rate (151) over its momentum distribution,

$$
\Gamma_{i}^{\mathrm{on}}\left(T, \mu_{q}\right)=\frac{d_{i}}{n_{i}^{\mathrm{on}}\left(T, \mu_{q}\right)} \int \frac{d^{3} p_{i}}{(2 \pi)^{3}} f_{i}\left(E_{i}, T, \mu_{q}\right) \Gamma_{i}^{\mathrm{on}}\left(\mathbf{p}_{i}, T, \mu_{q}\right)
$$

with the on-shell density of parton $i$ at finite $T$ and $\mu_{B}$ given by Eq. (59).

Figure 2.8 gives an overview of the relaxation time of a quark (left) and gluon (right) as a function of the scaled temperature $T / T_{c}\left(\mu_{B}\right)$ and the chemical potential $\mu_{B}$. The averaged relaxation times $\tau_{i}$ for quarks and antiquarks have a similar $T$-dependence: $\tau_{i}$ decreases with increasing temperature and stays almost constant at high $T$ as expected since $\sigma_{\text {tot }} \sim 1 / T^{3}$ and $n \sim T^{3}$ for large $\mathrm{T}$. The gluon relaxation time is about $0.3-0.4 \mathrm{fm} / \mathrm{c}$ in the region $1.5 T_{c} \leq T \leq 3 T_{c}$, which is significantly smaller than the quark relaxation time, which is about $1.0-1.5 \mathrm{fm} / \mathrm{c}$. Since the transport coefficients are directly proportional to the relaxation times, it is clear that the main contribution to the transport coefficients in the RTA comes from quarks and antiquarks. The relaxation times evaluated by Eq. (60) increase slightly with $\mu_{B}$ due to a decrease of the interaction rates $\Gamma_{i}$. A similar statement can be made if the spectral width $\gamma_{i}$ is used instead of the interaction rate in Eq. (61).

\subsubsection{Shear and bulk viscosities}

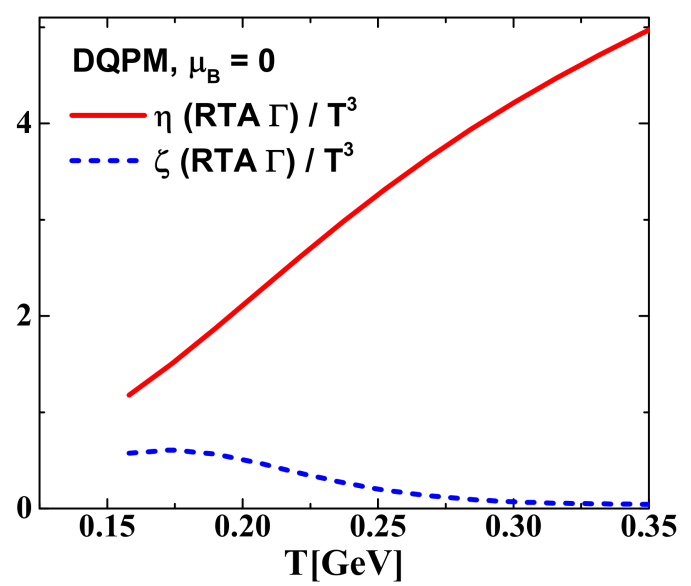

Figure 2.9: Ratio of shear $\eta / T^{3}$ and bulk $\zeta / T^{3}$ viscosities to the temperature cubed as a function of temperature $T$ for $\mu_{B}=0$. The solid red line and the dashed blue line show the DQPM results for the shear and bulk ratios accordingly, using the parton interaction rate $\Gamma_{i}(\mathbf{p}, T, \mu)$ for the relaxation time.

We begin with the most common transport coefficients for hydrodynamic simulations - the shear and bulk viscosities. The viscosities of QCD matter have been studied in a variety of models in the confined and deconfined phases. The shear viscosity provides information about the strength of the interaction within the QCD matter. In particular, in the framework of kinetic theory, it can be related to the interaction rates of hadrons or partons, which poses a challenge in first-principles evaluation. A wealth of theoretical model predictions shows that the temperature dependence of the QCD shear viscosity 
over the entropy density $\eta / s$ is qualitatively different for the two phases. Starting from the hadronic phase below the phase transition $T<T_{c}, \eta / s$ increases with temperature because the interaction weakens in the high temperature region. Approaching the phase transition from the hadronic to the QGP phase at vanishing chemical potential, $\eta / s$ has a broad dip followed by an increase with temperature. A similar property of the temperature dependence of the specific shear viscosity $\eta / s$ is seen for other fluids such as $\mathrm{H}_{2} \mathrm{O}, \mathrm{He}$ and $N_{2}[79,80]$.

The specific bulk viscosity of QGP matter is predicted to be low, however, it is expected to be finite near the phase transition [42]. The presence of bulk viscosity reduces the rate of radial expansion of the fluid and thus affects the mean momentum of the generated particles. For conformal fluids the bulk viscosity is known to be identically zero, and the unbounded QCD medium is expected to adopt conformal behaviour in the high energy or temperature regime. Nevertheless, as it has been shown in Section 2.2 the 1QCD results on the enhanced trace anomaly close to $T_{c}$ indicate that it is probably not the case for the QGP medium in the vicinity of the phase transition.

The shear viscosity to entropy density ratio $\eta / s$ for the QGP medium was predicted to be very low $[29,73]$. While the ratios $\eta / s$ and $\zeta / s$ are expected to be small the shear viscosity $\eta$ as well as the entropy $s$ of partonic system are high and scale with the temperature as $\sim T^{3}$ as shown in Fig. 2.9. In the RTA the shear viscosity reads [76]:

$$
\eta^{\mathrm{RTA}}\left(T, \mu_{B}\right)=\frac{1}{15 T} \sum_{i=q, \bar{q}, g} \int \frac{d^{3} p}{(2 \pi)^{3}} \frac{\mathbf{p}^{4}}{E_{i}^{2}} \tau_{i}\left(\mathbf{p}, T, \mu_{B}\right) d_{i}\left(1 \pm f_{i}\right) f_{i},
$$

where $d_{q}=2 N_{c}=6$ and $d_{g}=2\left(N_{c}^{2}-1\right)=16$ denote degeneracy factors for spin and color in case of quarks and gluons, $\tau_{i}$ are the relaxation times. In extension to our previous studies in Refs. [47,81] we here include the Pauli-blocking and Bose enhancement factors, respectively.

One way to evaluate the viscosity coefficients of partonic matter is the Kubo formalism $[82,83]$, which was used to calculate the viscosities for a previous version of the DQPM within the PHSD transport approach in a box with periodic boundary conditions (cf. Ref. [84]). The current values for the total $\eta / s$ ratio can be qualitatively compared with the gluodynamic lattice calculations of QCD at $\mu_{B}=0$ from Ref. [85]. The gluodynamic calculation in the DQPM was performed in Ref. [46], where the ratio $\eta_{g} / s_{g} \sim 0.2$ is in good agreement with the presented lattice QCD results. We note that the $\eta / s$ ratio does not vary strongly with $\mu_{B}$ and exhibits a similar behavior as a function of temperature for all $\mu_{B}$ considered. The RTA estimate of the shear viscosity is found to be very close to the one from the Kubo formalism [1] indicating that the quasiparticle limit $(\gamma \ll M)$ holds in the DQPM.

The ratio of the shear viscosity to entropy density at $\mu_{B}=0$ is shown in Fig. 2.10 (a) in comparison to the lattice QCD calculation for $N_{f}=0$ from Ref. [85]. The ratio $\eta / s$ at $\mu_{B}=0$ also is in a good agreement with the predictions from a Bayesian analysis of experimental heavy-ion data from Ref. [86]. In extension to Ref. [1] we also show the separate contributions of various quasiparticle species to the shear viscosity $\eta=\eta_{q, \bar{q}}+$ 

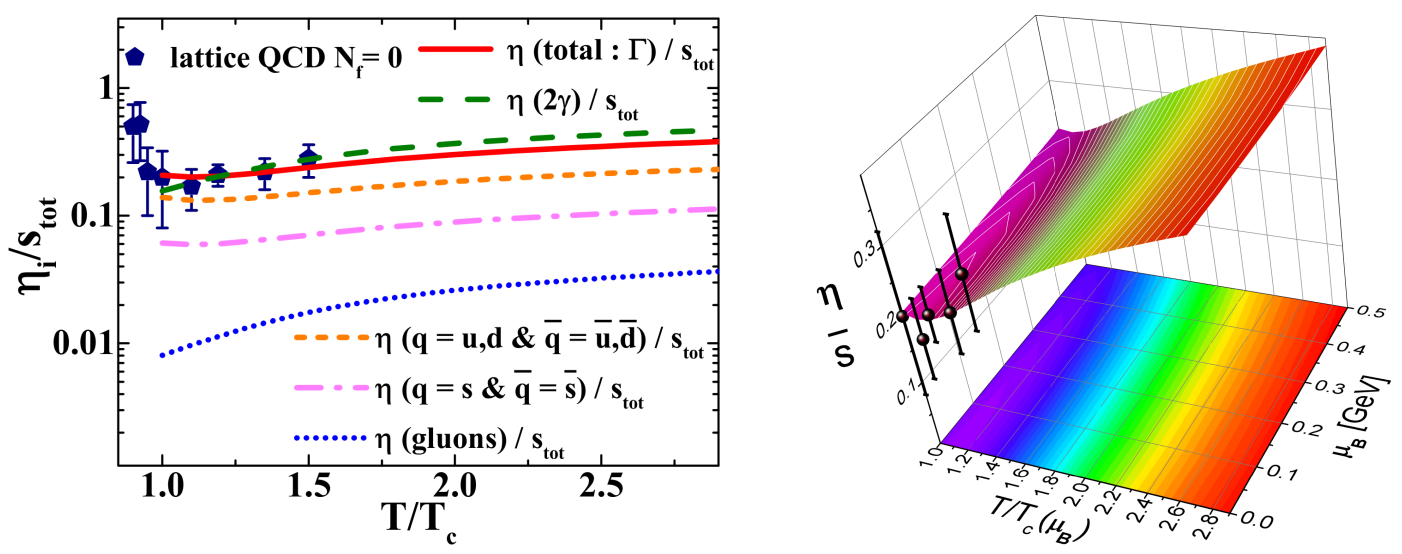

Figure 2.10: Specific shear viscosity $\eta / s$ (left) as a function of the scaled temperature $T / T_{c}$ for $\mu_{B}=0$ and (right) for moderate chemical potentials $\mu_{B} \leq 0.5 \mathrm{GeV}$ as a function of the scaled temperature $T / T_{c}\left(\mu_{B}\right)$ and the baryon chemical potential $\mu_{B}$. The lines shows the DQPM result for $\eta_{i} / s_{\text {tot }}$ from Eq. (63) using the interaction rate $\Gamma_{i}(\mathbf{p}, T, \mu)$ for different quasiparticle species: light quarks and anti-quarks (short dashed orange line), strange quarks and anti-quarks (dot-dashed magenta line), gluons (dotted blue line). The solid red line and the dashed green line show the DQPM results for total ratios of viscosity to entropy density using the parton interaction rate $\Gamma_{i}(\mathbf{p}, T, \mu)$ and the spectral width $2 \gamma_{i}(T, \mu)$ for the relaxation time. The pentagons show the lQCD prediction for pure $\mathrm{SU}(3)$ gauge theory taken from Ref. [85].

$\eta_{g}, q=u, d, s$ divided by the total entropy density $s_{\text {tot }}^{\mathrm{DQPM}}=s_{q, \bar{q}}+s_{g} \eta_{i} / s_{\text {tot }}$ : light quarks and anti-quarks (short dashed orange line), strange quarks and anti-quarks (dot-dashed magenta line), gluons (dotted blue line). The solid red line corresponds to the ratio of the total shear viscosity to the entropy density. As expected, smaller values of the shear viscosity are observed for the gluons than for the quarks, since the relaxation time of the gluons is about twice that of the quarks and the masses of the gluons are about half that of the quarks, which enters into the shear viscosity via the factor $1 / E_{i}^{2}$. The light quarks and anti-quarks make the main contribution to the total viscosity $\sim 60 \%$. The strange quarks and anti-quarks contribute $\sim 30 \%$, while the contribution of the gluons is about $\sim 10 \%$. The differences in the shear viscosity of the different quark flavours are mainly due to the mass difference. We mention that the hierarchy obtained here is shown for the ratio of shear viscosity for each quasiparticle species to the total entropy density $\eta_{i} / s_{t o t}$, while in the recent calculations in the quasiparticle model of Ref. [53] for $\mu_{B}=0$ the ratios of shear viscosity for each quasiparticle species to the entropy density of the considered quasiparticle species $\eta_{i} / s_{i}$ were shown. It is worth noting that in Ref. [53] only the on-shell case is considered, where quasiparticles have no widths and the couplings are higher than in the DQPM, leading to an increase in the relaxation times and the shear viscosity to entropy density ratio $\eta / s$.

The bulk viscosity of the partonic phase can be similarly expressed via relaxation times 

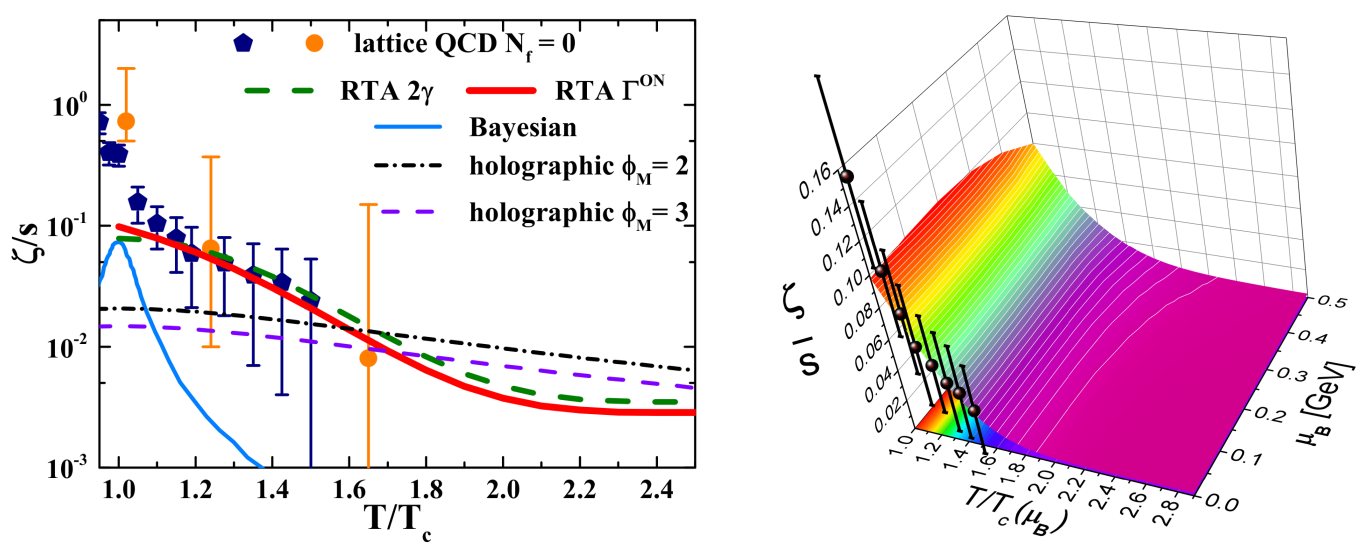

Figure 2.11: Specific bulk viscosity to entropy density $\zeta / s$ (left) as a function of the scaled temperature $T / T_{c}$ for $\mu_{B}=0$ and (right) for non-zero $\mu_{B}$ as a function of the scaled temperature $T / T_{c}\left(\mu_{B}\right)$ and the baryon chemical potential $\mu_{B}$. The solid red line and the dashed green line show the DQPM results within the RTA from Eq. (64) using the parton interaction rate $\Gamma_{i}(\mathbf{p}, T, \mu)$ and the spectral width $2 \gamma_{i}(T, \mu)$ for the relaxation time. The symbols display the lQCD data for $N_{f}=0$ pure SU(3) gauge theory taken from Refs. [87] (pentagons) and [88] (circles). The solid blue line shows the estimate from a Bayesian analysis of experimental heavy-ion data taken from Ref. [86].

within the RTA following Ref. [76]:

$$
\begin{gathered}
\zeta^{\mathrm{RTA}}\left(T, \mu_{B}\right)=\frac{1}{9 T} \sum_{i=q, \bar{q}, g} \int \frac{d^{3} p}{(2 \pi)^{3}} \tau_{i}\left(\mathbf{p}, T, \mu_{B}\right) \\
\times \frac{1}{E_{i}^{2}}\left(\mathbf{p}^{2}-3 c_{s}^{2}\left(E_{i}^{2}-T^{2} \frac{d m_{i}^{2}}{d T^{2}}\right)\right)^{2} d_{i}\left(1 \pm f_{i}\right) f_{i},
\end{gathered}
$$

where $c_{s}^{2}$ is the speed of sound squared, $\frac{d m_{i}^{2}}{d T^{2}}$ is the DQPM parton mass derivative which becomes large close to the critical temperature $T_{c}$. The DQPM results for the viscosities over temperature cubed are showed in Fig. 2.9, where the parton interaction rate $\Gamma_{i}\left(\mathbf{p}, T, \mu_{B}\right)$ was used for the relaxation time. The solid red line corresponds to the ratio of the shear viscosity to the temperature cubed $\eta / T^{3}$, while the dashed blue line shows the ratio of the bulk viscosity to the temperature cubed $\zeta / T^{3}$. The specific bulk viscosity $\zeta / s$ as a function of the scaled temperature $T / T_{c}$ at vanishing chemical potential is shown in Fig. 2.11 (left). The solid red line $\left(\zeta_{\Gamma^{\mathrm{on}}}^{\mathrm{RTA}} / s\right)$ displays the results from Eq. (64) using the interaction rate $\Gamma_{i}(\mathbf{p}, T, \mu)$ while the dashed green line shows the same result in the relaxation-time approximation (64) by replacing $\Gamma_{i}$ by the spectral width $2 \gamma_{i}$. The symbols correspond to the lQCD data for pure SU(3) gauge theory taken from Refs. [87](pentagons) and [88](circles). The solid blue line displays the results from a Bayesian analysis of experimental heavy-ion data from Ref. [89]. The bulk viscosities of our quasiparticle models are always smaller than the shear viscosities even close to the phase transition. The DQPM results for $N_{f}=2+1$ surprisingly coincide with the gluodynamic lattice data from Ref. [87], except in the vicinity of $T_{c}$, as expected, since in gluodynamics 1st order phase transition takes place while in the DQPM $N_{f}=2+1$ a crossover is encoded. The lattice points close to $T_{c}$ have large error bars since lattice simulations at low temperatures require much 
larger statistics than simulations at higher $T$. We compare the bulk viscosity also to predictions from a Bayesian analysis of experimental data from Refs. [86, 89]. The DQPM exhibits the expected peak close to the critical temperature which is close to the Bayesian line maximum of the peak at $T_{c}$ with $\zeta / s \simeq 0.075$. The ratio from the Bayesian analysis shows a sudden drop to zero, which is incompatible with the small, but non-zero lattice values. Furthermore, the DQPM results for viscosities are in a good agreement with the gluodynamic lattice QCD calculation at $\mu_{B}=0$ from Ref. [87]. In the case of the bulk viscosity $\zeta$ we have found that the original DQPM calculations are very close to the results obtained using the interaction rates, such that they merge as one can see in Fig. 2.11 (left). Results of $\zeta / s$ for moderate baryon chemical potentials are presented in Fig. 2.11 (right) as a function of the scaled temperature and $\mu_{B}$. Comparing $\mu_{B}$-dependence of specific shear and bulk viscosities from the DQPM we see that the ratio $\eta / s$ increases with $\mu_{B}$ at all temperatures, while $\zeta / s$ only for $T>1.2 T_{c}$. The specific bulk viscosity decreases in the vicinity of $T_{c}$, where it is dominated by the mean-field effects that enter as $d M^{2} / d T^{2}$ and pronounced temperature dependence of the speed of sound squared. As we discussed above, the DQPM the masses depend primarily on the effective coupling $g^{2}$, which decreases as a function of $\mu_{B}$, also the mean-field effects become weaker. This causes a small decrease of $\zeta / s$ in contrast to the $\eta / s$. At higher temperatures the mean-field effects become also less pronounced, resulting in a decreasing $\zeta / s$ as a function of temperature. That clarifies why the $\mu_{B}$ behavior of the bulk viscosity changes with temperature. When the mean-field effects become subleading, their further decrease has no influence on the bulk viscosity and the ratio $\zeta / s$ starts to increase with $\mu_{B}$.

\subsubsection{Electric conductivity}

Another important transport coefficient is the electric conductivity for stationary electric fields $\sigma_{Q}\left(T, \mu_{B}\right)$, which describes the response of the system to an external electric field. The study of the temperature and baryon chemical potential dependencies of $\sigma_{Q}\left(T, \mu_{B}\right)$ is of fundamental importance for the possible generation of the chiral-magnetic effect in predominantly peripheral heavy-ion reactions. Moreover, electric conductivity affects the emission rate of soft photons [90] as well as their spectra [91-93]. The electric conductivity $\sigma_{Q}$ is evaluated by using the relaxation time approximation $[76,78]$ :

$$
\sigma_{Q}^{\mathrm{RTA}}\left(T, \mu_{B}\right)=\frac{e^{2}}{3 T} \sum_{i=q, \bar{q}} q_{i}^{2} \int \frac{d^{3} p}{(2 \pi)^{3}} \frac{\mathbf{p}^{2}}{E_{i}^{2}} \cdot \tau_{i}\left(\mathbf{p}, T, \mu_{B}\right) d_{i}\left(1-f_{i}^{(0)}\right) f_{i}^{(0)} .
$$

Here $q_{i}=+2 / 3,-1 / 3,-1 / 3$ are the dimensionless electric charges of $\mathrm{u}, \mathrm{d}, \mathrm{s}$ quarks (see Table 1$), d_{q}=2 N_{c}=6$ are degeneracy factors for spin and color in case of quarks and antiquarks, $\tau_{i}$ their relaxation times, and $f_{i}^{(0)}$ denote the Fermi-Dirac distribution functions for quark and anti-quarks defined by Eq. (58). In these formulae we deal only with quarks and anti-quarks of $N_{f}=3$ flavours. Each parton has a contribution proportional to its charge squared. Unlike viscosities, the electric conductivity doesn't contain a contribution from gluons.

Figure 2.12 depicts the results for $\sigma_{Q} / T$ a) as a function of the scaled temperature 

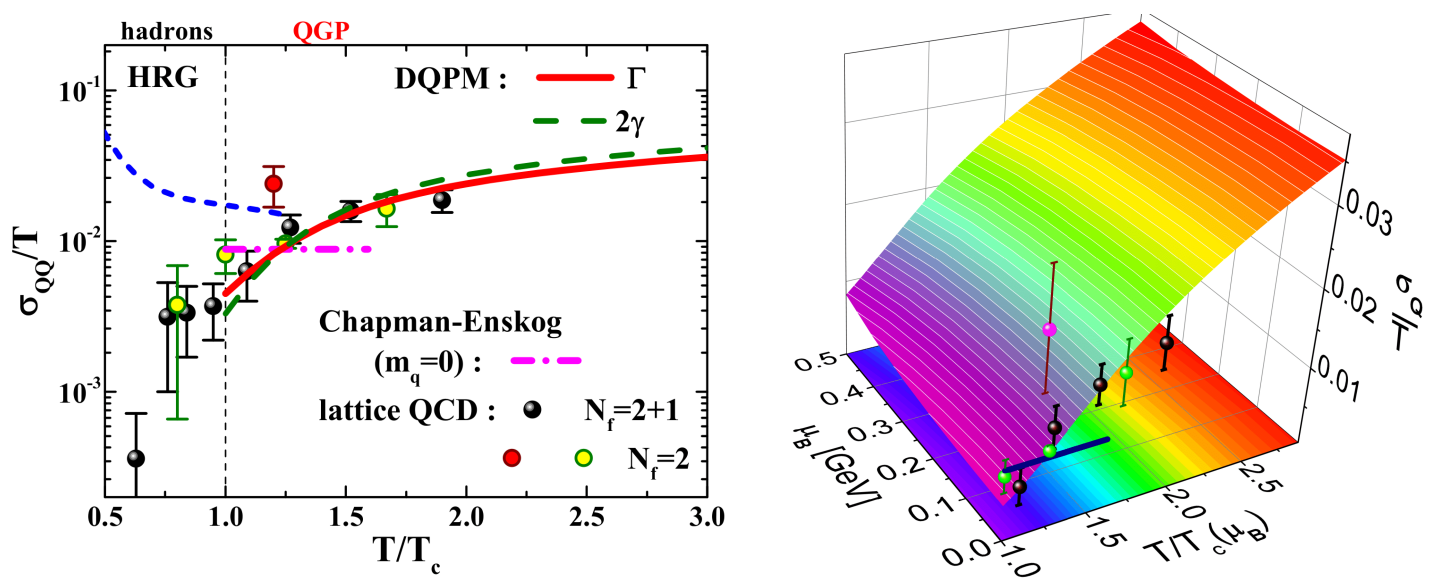

Figure 2.12: Scaled electric conductivity $\sigma_{Q} / T$ (left) as a function of the scaled temperature $T / T_{c}$ for $\mu_{B}=0$ and (right) for non-zero $\mu_{B}$ as a function of the scaled temperature $T / T_{c}\left(\mu_{B}\right)$ and the baryon chemical potential $\mu_{B}$. The solid red line and the dashed green line show the DQPM results within the RTA from Eq. (65) using the parton interaction rate $\Gamma_{i}(\mathbf{p}, T, \mu)$ and the spectral width $2 \gamma_{i}(T, \mu)$ for the relaxation time. The symbols display lQCD data for $N_{f}=2$ taken from Refs. [94,95] (red circles with brown borders), (yellow circles with green borders) and for $N_{f}=2+1$ taken from Refs. [83,96] (spheres). The dot-dashed magenta line corresponds to the results from the first order Chapman-Enskog approximation taken from Ref. [52].

$T / T_{c}$ and b) the $\mu_{B}$ and $T / T_{c^{-}}$-dependencies from the DQPM. The solid red line shows the DQPM result $\sigma_{Q}^{\mathrm{RTA}} / T$ according to Eq. (65) using the interaction rate $\Gamma_{i}(\mathbf{p}, T, \mu)$ while the dashed green line shows the result in the relaxation-time approximation (65) by replacing $\Gamma_{i}$ by the spectral width $2 \gamma_{i}(T, \mu)$. We note that two results for $\sigma_{Q} / T$ are in good agreement. The differences between the two lines can vary by less than $14 \%$ except near $T_{c}$, where the momentum dependence of the relaxation times may play a role. This similarity shows again that the effective widths in the parton propagators - which provide the spectral widths of the partons - are in good agreement with the microscopic collision rates.

The ratio of electric conductivity to the temperature squared increases quadratically with temperature near $T_{c}$ and linearly at high temperatures, which can be explained by the increasing number of quarks and by the temperature dependence of the quark relaxation times. An increase in the number of charge carriers leads to a growth of the electric current and, thus, to a rising conductivity. Averaged over momenta the quark relaxation time decreases with increasing temperature and stays almost constant at high $T$ and increases with increasing $\mu_{B}$ (see Fig. 2.8). For temperatures $T>2.5 T_{C}$ this leads to a roughly linear dependence in temperature, $\sigma_{Q}^{\mathrm{RTA}} / T \sim T$.

An alternative way to evaluate the electric conductivity is to solve the relativistic transport equations for partons in a box with periodic boundary conditions in the presence of an external electric field as in Refs. [97,98]. It is interesting to compare to the results of the Chapman-Enskog method, where quarks and gluons are considered massless as in Ref. [52], while scattering cross sections are fixed in order to descibe the Kovtun-SonStarinets bound for the shear viscosity to entropy density ratio $(\eta / s)_{\mathrm{KSS}}=1 /(4 \pi)[99]$, 
leading to $\sigma_{\text {tot }} \approx 0.72 / T^{2}$. Besides the completely different treatment of microscopic degrees of freedom - masses of the partons and scattering cross-sections differ significantly between the two methods - a good agreement between the results is found in the vicinity of $T_{c}$. Furthermore, we compare to the results from the non-conformal holographic model [49], which are close to the DQPM results in the vicinity of the transition $T_{c}-1.5 T_{c}$, however, the temperature dependence of the $\sigma_{Q} / T$ differs and the values at high temperatures are above the DQPM predictions.

\subsubsection{Baryon diffusion}

In the region of finite baryon chemical potentials it is important to consider other transport coefficients that are thought to be more sensitive to the net baryon density of the system, such as the baryon diffusion coefficient. This transport coefficient provides information about the response to inhomogeneities in the baryon density. The baryon diffusion coefficient regulates the dissipative part of the baryon flux, which can be expressed as follows:

$$
\delta J_{B}^{\mu}=\kappa_{B} D^{\mu}\left(\frac{\mu_{B}}{T}\right),
$$

where $\kappa_{B}$ is the baryon diffusion coefficient, $D^{\mu}=d^{\mu}-u^{\mu} u^{\nu} d_{\nu}$ is the transverse gradient, while $u^{\mu}$ is the local fluid velocity. The dissipative baryon current can be related to the heat flow as $q^{\mu}=-\frac{\epsilon+p}{n_{B}} \delta J_{B}^{\mu}[100,101]$. On the other hand, the heat flow reads

$$
q^{\mu}=\lambda \frac{n_{B}}{\epsilon+p} D^{\mu}\left(\frac{\mu_{B}}{T}\right)
$$

where $\lambda$ is the heat conductivity. Thus we can obtain a relation between the heat conductivity and the diffusion coefficient:

$$
\kappa_{B}=\lambda\left(\frac{n_{B} T}{\epsilon+p}\right)^{2}
$$

One can easily estimate that the two coefficients $\kappa_{B}$ and $\lambda$ differ by two orders of magnitude.

The baryon diffusion coefficient in the RTA can be expressed as:

$$
\begin{aligned}
\kappa_{B}^{\mathrm{RTA}}\left(T, \mu_{B}\right) & =\frac{1}{3} \sum_{i=q, \bar{q}} \int \frac{d^{3} p}{(2 \pi)^{3}} \mathbf{p}^{2} \tau_{i}\left(\mathbf{p}, T, \mu_{B}\right) \\
& \times \frac{1}{E_{i}^{2}}\left(b_{a}-\frac{n_{B} E_{i}}{\epsilon+p}\right)^{2} d_{i}\left(1 \pm f_{i}\right) f_{i} .
\end{aligned}
$$

Here $b_{i}= \pm 1 / 3$ is the baryon number of quark and antiquark, $n_{B}$ is the baryon density, $w=\epsilon+p$ is the enthalpy. Taking into account relation (68) one can see that Eq. (69) is in agreement with the RTA expression for the heat conductivity as derived in Ref. [76].

Figure 2.13 shows the actual results for the baryon diffusion coefficent in the range of temperature and non-zero baryon chemical potential $\mu_{B}$. We compare the DQPM results to the estimates based on the thermal conductivity results from the AdS/CFT 

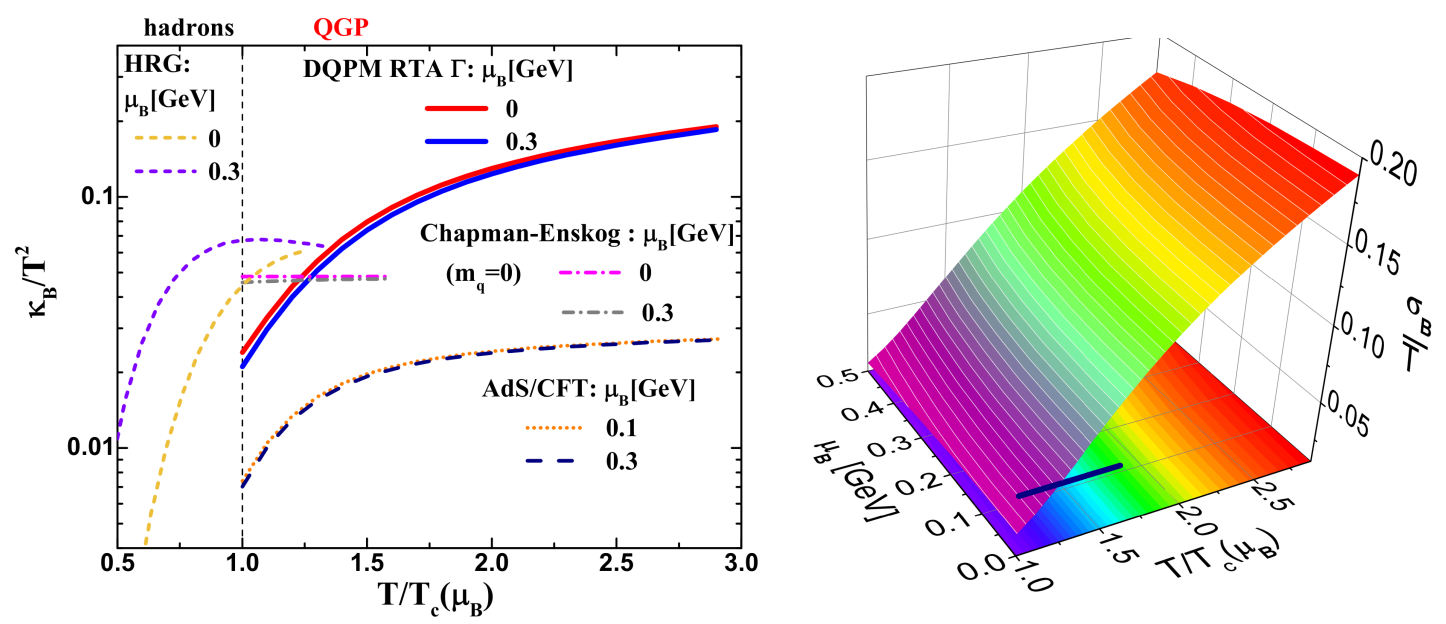

Figure 2.13: Ratio of the baryon diffusion coefficent to the temperature squared $\kappa_{B} / T^{2} \equiv$ $\sigma_{B} / T$ (left) as a function of scaled temperature for a fixed baryon chemical potential $\mu_{B}=0$ and $0.3 \mathrm{GeV}$ and (right) as a function of scaled temperature for moderate baryon chemical potentials $\mu_{B} \leq 0.5 \mathrm{GeV}$. The dashed lines represent the AdS/CFT results for $\kappa_{B}^{S S}$, which are obtained using the results from Ref. [101] and the DQPM EoS. The dot-dashed lines correspond to the results from the first order Chapman-Enskog approximation taken from Ref. [52]. The light green dotted line and dashed black line correspond to the results from the Einstein-Maxwell-Dilaton (EMD) holographic model [49].

correspondence [102]. Using the relation between the baryon diffusion coefficient and the heat conductivity (68), we can translate these results to the following expression:

$$
\kappa_{B}^{A d S / C F T}=2 \pi \frac{T s}{\mu_{B}^{2}}\left(\frac{n_{B} T}{\epsilon+p}\right)^{2}
$$

Here $s$ is the entropy density, $n_{B}$ is the baryon density, $w=\epsilon+p$ is the enthalpy. We have calculated $\kappa_{B}^{A d S / C F T}$ using $s, n_{B}, \epsilon$ and $p$ from the DQPM.

In the vicinity of $T_{c}$ the DQPM values for the diffusion coefficient are in agreement with the calculations within the Chapman-Enskog first-order approximation using cross-sections for massless quarks and gluons in Ref. [52]. However, for higher temperatures the ratio $\kappa_{B}^{\mathrm{RTA}} / T^{2}$ increases with temperature in the DQPM while the Chapman-Enskog method can not reproduce this groth and predicts approximately constant behaviour $\kappa_{B}^{\mathrm{CE}} / T^{2} \sim 0.048$ for all $T$. It is expected that $\kappa_{B}$ has a more pronounced $\mu_{B}$-dependence than another transport coefficients since it depends explicitly on the baryon density, and we see a slight decrease of the ratio $\kappa_{B}^{\mathrm{RTA}} / T^{2}$ for the DQPM with increasing chemical potential $\mu_{B}$, while results from other approaches $\kappa_{B}^{\mathrm{CE} / T^{2} \sim 0.048}$ and $\kappa_{B}^{A d S / C F T}$ are approximately $\mu_{B}$ independent for the considered region of $\mu_{B}$. The similar slight decrease has been predicted in the holographic calculations in Ref. [49].

It is worth noting that the $\mu_{B}$-dependence of $\kappa_{B}$ can be an interesting property of the partonic phase, which may have effects on observables: it has been found within the hybrid (hydrodynamics + transport) theoretical framework in Ref. [103] that the baryon diffusion enhances the difference between proton and antiproton mean transverse momenta and elliptic flow $v_{2}\left(p_{T}\right)$. The baryon decreases the proton elliptic flow while increasing 
anti-proton elliptic flow.

\subsubsection{Diffusion coefficient matrix: calculations within the RTA and the Chapman-Enskog approximation}

In the previous Subsections we have addressed the diffusion coefficients of the conserved baryon number and electric charge. Generally one should consider the full diffusion coefficient matrix $\left(\kappa_{q q^{\prime}}\right.$, where $\left.q, q^{\prime}=\mathrm{B}, \mathrm{S}, \mathrm{Q}\right)$, which was introduced and evaluated for a hadron gas and a simple model for the QGP $[52,104]$. Since quarks carry more than one of the conserved charges, the diffusion current of each charge is no longer exclusively proportional to the gradient of the thermal potential $\nabla^{\mu} \mu_{q} / T$ of that specific charge. Instead, mixing of the currents occurs, with the gradients of each individual charge density capable of producing a diffusion current of any other charge. Full diffusion coefficient matrix can be written as follows:

$$
\left(\begin{array}{c}
j_{B}^{\mu} \\
j_{Q}^{\mu} \\
j_{S}^{\mu}
\end{array}\right)=\left(\begin{array}{ccc}
\kappa_{B B} & \kappa_{B Q} & \kappa_{B S} \\
\kappa_{Q B} & \kappa_{Q Q} & \kappa_{Q S} \\
\kappa_{S B} & \kappa_{S Q} & \kappa_{S S}
\end{array}\right) \cdot\left(\begin{array}{c}
\nabla^{\mu} \alpha_{B} \\
\nabla^{\mu} \alpha_{Q} \\
\nabla^{\mu} \alpha_{S}
\end{array}\right)
$$

These investigations were followed by a more extended study in the hadronic phase from kinetic theory in the case of the electric cross-conductivities [105]. Furthermore, a first study on the impact of the coupling of baryon number and strangeness was provided in Ref. [104]. To this aim, the matrix of diffusion coefficients of hot and dense nuclear matter must be thoroughly investigated, as it is important for accurate hydrodynamic simulations. It has been further motivated that the off-diagonal coefficients may have implications for the chemical composition of the hadronic phase [105]. We here combine the developments of Refs. $[2,52,104]$ and evaluate the diffusion coefficient matrix of the strongly interacting non-perturbative QGP at finite $\left(T, \mu_{\mathrm{B}}\right)$, with properties described by the DQPM model, based on a recently explored the Chapman-Enskog study $[52,104,106]$. This allows us to explore the influence of traces of non-equilibrium effects by accounting for the higher modes of the distribution function on the transport properties and compare the results with the often used kinetic RTA approximation. We consider the $\left(T, \mu_{\mathrm{B}}\right)$-dependence of the diffusion coefficients $\kappa_{q q^{\prime}}$ for $q, q^{\prime}=\mathrm{B}, \mathrm{S}, \mathrm{Q}$ charges for baryon chemical potentials $\mu_{\mathrm{B}} \leq 0.5 \mathrm{GeV}$.

We review first results for the diffusion coefficient matrix for the hot quark-gluon plasma at zero and finite baryon chemical potential $\mu_{\mathrm{B}}$ by applying the Chapman-Enskog method, described in detail in Refs. $[52,104,106]$, to a strongly interacting QGP system described by the DQPM. This represents a significant and important improvement over the "simplified" model of a partonic system used in Refs. $[52,104,106]$. The Chapman-Enskog results are compared with the results for the diffusion coefficient matrix computed within the RTA approach based on the DQPM, and with several other models. The fact that the linearized Boltzmann equation is solved in the framework of $\mathrm{CE}$ implies an improvement over approaches using the RTA [104] in terms of accounting for higher moments of the 
distribution function. However, the proposed Chapman-Enskog method requires a few approximations for the QGP description, which are not considered for the RTA estimates within the DQPM, in particular:

1. The system is assumed to obey classical (Maxwell-Boltzmann) statistics, i.e. $a_{i}=0$ for all particle species in Eq. (58).

2. All particles are on-shell, therefore only the pole-masses from the DQPM, which depend on temperature and baryon-chemical potential, are assumed but their widths are neglected.

3. Inelastic scattering channels are neglected. That implies that flavor-changing processes are not taken into consideration, i.e. $q \bar{q} \rightarrow q^{\prime} \bar{q}^{\prime}$ are not allowed.

4. All scattering processes are considered to be isotropic. We therefore feed integrated total cross sections $\sigma_{\text {tot }}^{i j \rightarrow a b}$ into the CE evaluation which are evaluated from the anisotropic differential cross section from the DQPM via Eq. (43). The dependence on $\sqrt{s}$, temperature and baryon-chemical potential is taken into account, $\sigma_{\text {tot }}^{i j \rightarrow i j} \equiv$ $\sigma_{\text {tot }}^{i j \rightarrow i j}\left(\sqrt{s}, T, \mu_{\mathrm{B}}\right)$.

We note that the CE method can in principle be improved such that approximations (1) and (3) become unnecessary. A comparison of the two methods for the simplistic case of constant cross sections is presented in Appendix A, where we find indication that approximation (3) may have a non-negligible impact. Such improvements can be done in future work. However, the nature of the method makes a further improvement of points (2) and (4) difficult and requires further detailed study.

Following the steps taken in Refs. $[52,104,106]$, we can express the diffusion coefficient matrix for a classical system under the assumption of elastic isotropic scattering processes as,

$$
\kappa_{q q^{\prime}}=\frac{1}{3} \sum_{i=1}^{N_{\text {species }}} q_{i} \sum_{m=0}^{M} \lambda_{m, q^{\prime}}^{(i)} \int_{\mathbb{R}^{3}} \mathrm{~d} K_{i} E_{i, \mathbf{k}}^{m}\left(m_{i}^{2}-E_{i, \mathbf{k}}^{2}\right) f_{i, \mathbf{k}}^{(0)},
$$

where we use the notation $\mathrm{d} K_{i} \equiv \frac{\mathrm{d}^{3} \mathbf{k}_{i}}{(2 \pi)^{3} E_{i, \mathbf{k}}}, q_{i}$ denotes the conserved charge, which is baryon, electric or strange charge. In Eq. (72) the coefficients $\lambda_{m, q^{\prime}}^{(i)}$ are solutions of the linearized Boltzmann equation in the form $[52,104,106]$ :

$$
\sum_{m=0}^{M} \sum_{j=1}^{N_{\text {species }}}\left(\mathcal{A}_{n m}^{i} \delta^{i j}+\mathcal{C}_{n m}^{i j}\right) \lambda_{m, q}^{(j)}=b_{q, n}^{i}
$$


with quantities

$$
\begin{aligned}
& \mathcal{A}_{n m}^{i} \equiv \sum_{\ell=1}^{N_{\text {species }}} \gamma_{i \ell} \int \mathrm{d} K_{i} \mathrm{~d} K_{\ell}^{\prime} \mathrm{d} P_{i} \mathrm{~d} P_{\ell}^{\prime}(2 \pi)^{6} s\left(\frac{\mathrm{d}}{\mathrm{d} \Omega} \sigma_{i \ell \rightarrow i \ell}\right) \\
& \delta^{(4)}\left(k_{i}+k_{\ell}^{\prime}-p_{i}-p_{\ell}^{\prime}\right) f_{i, \mathbf{k}}^{(0)} f_{\ell, \mathbf{k}^{\prime}}^{(0)} E_{i, \mathbf{k}}^{n-1} k_{i,\langle\alpha\rangle}\left(E_{i, \mathbf{p}}^{m} p_{i}^{\langle\alpha\rangle}-E_{i, \mathbf{k}}^{m} k_{i}^{\langle\alpha\rangle}\right), \\
& \mathcal{C}_{n m}^{i j} \equiv \gamma_{i j} \int \mathrm{d} K_{i} \mathrm{~d} K_{j}^{\prime} \mathrm{d} P_{i} \mathrm{~d} P_{j}^{\prime}(2 \pi)^{6} s\left(\frac{\mathrm{d}}{\mathrm{d} \Omega} \sigma_{i j \rightarrow i j}\right) \\
& \delta^{(4)}\left(k_{i}+k_{j}^{\prime}-p_{i}-p_{j}^{\prime}\right) f_{i, \mathbf{k}}^{(0)} f_{j, \mathbf{k}^{\prime}}^{(0)} E_{i, \mathbf{k}}^{n-1} k_{i,\langle\alpha\rangle}\left(E_{j, \mathbf{p}^{\prime}}^{m} p_{j}^{\prime\langle\alpha\rangle}-E_{j, \mathbf{k}^{\prime}}^{m} k_{j}^{\prime\langle\alpha\rangle}\right), \\
& b_{q, n}^{i} \equiv \int_{\mathbb{R}^{3}} \mathrm{~d} K_{i} E_{i, \mathbf{k}}^{n-1}\left(m_{i}^{2}-E_{i, \mathbf{k}}^{2}\right)\left(\frac{E_{i, \mathbf{k}} n_{q}}{\epsilon+P_{0}}-q_{i}\right) f_{i, \mathbf{k}}^{(0)} .
\end{aligned}
$$

Additionally, imposing Landau's definition of the frame [107], leads to an another constraint:

$$
\begin{aligned}
& W^{\mu}=\sum_{i=1}^{N_{\text {species }}} \int_{\mathbb{R}^{3}} \mathrm{~d} K_{i} E_{i, \mathbf{k}} k_{i}^{\langle\mu\rangle} f_{i, \mathbf{k}}^{(1)} \stackrel{!}{=} 0 \Rightarrow \\
& \sum_{i=1}^{N_{\text {species }}} \sum_{m=0}^{M} \lambda_{m, q}^{(i)} \int_{\mathbb{R}^{3}} \mathrm{~d} K_{i} E_{i, \mathbf{k}}^{m+1}\left(m_{i}^{2}-E_{i, \mathbf{k}}^{2}\right) f_{i, \mathbf{k}}^{(0)} \stackrel{!}{=} 0 .
\end{aligned}
$$

Above we introduced the truncation order $M$, where for simplicity the order is fixed to $M=1$ which corresponds to the 14-moment approximation [108].

Following Ref. [52], the diffusion coefficient matrix in the RTA can be expressed as follows:

$$
\kappa_{q q^{\prime}}=\frac{1}{3} \sum_{i=1}^{N_{\text {species }}} \tau_{i} q_{i} \int \mathrm{d} K_{i} \frac{1}{E_{i, \mathbf{k}}}\left(m_{i}^{2}-E_{i, \mathbf{k}}\right)\left(\frac{E_{i, \mathbf{k}} n_{q^{\prime}}}{\epsilon+P_{0}}-q_{i}^{\prime}\right) f_{i, \mathbf{k}}^{(0)} \tilde{f}_{i, \mathbf{k}}^{(0)}
$$

where we use shorthand notation $\tilde{f}_{i, \mathbf{k}}^{(0)}=\left(1+\frac{a_{i}}{d_{i}} f_{i, \mathbf{k}}^{(0)}\right)$.

In Fig. 2.14 we show a comparison of the results from DQPM RTA and CE (DQPM) to a variety of model estimates for both, the partonic $[94,96,112,113,116,117]$ and hadronic phase $[52,104-106,114,115]$, at $\mu_{\mathrm{q}}=0$ in a temperature range between 0 and $3 T_{c}$, where here the deconfinement temperature is $T_{c}=158 \mathrm{MeV}$. The Chapman-Enskog and RTA results for the dimensionless ratio of electric conductivity to temperature $\sigma_{\mathrm{QQ}} / T$ (later referred to as scaled electric conductivity) for $\mu_{q}=0$ are presented in Fig.2.14 (left) as solid red and dashed black lines. Results for both methods have a similar increase with temperature, which is mainly a consequence of the temperature dependence of the cross section (as discussed before) and also of the increasing total electric charge density [104]. 

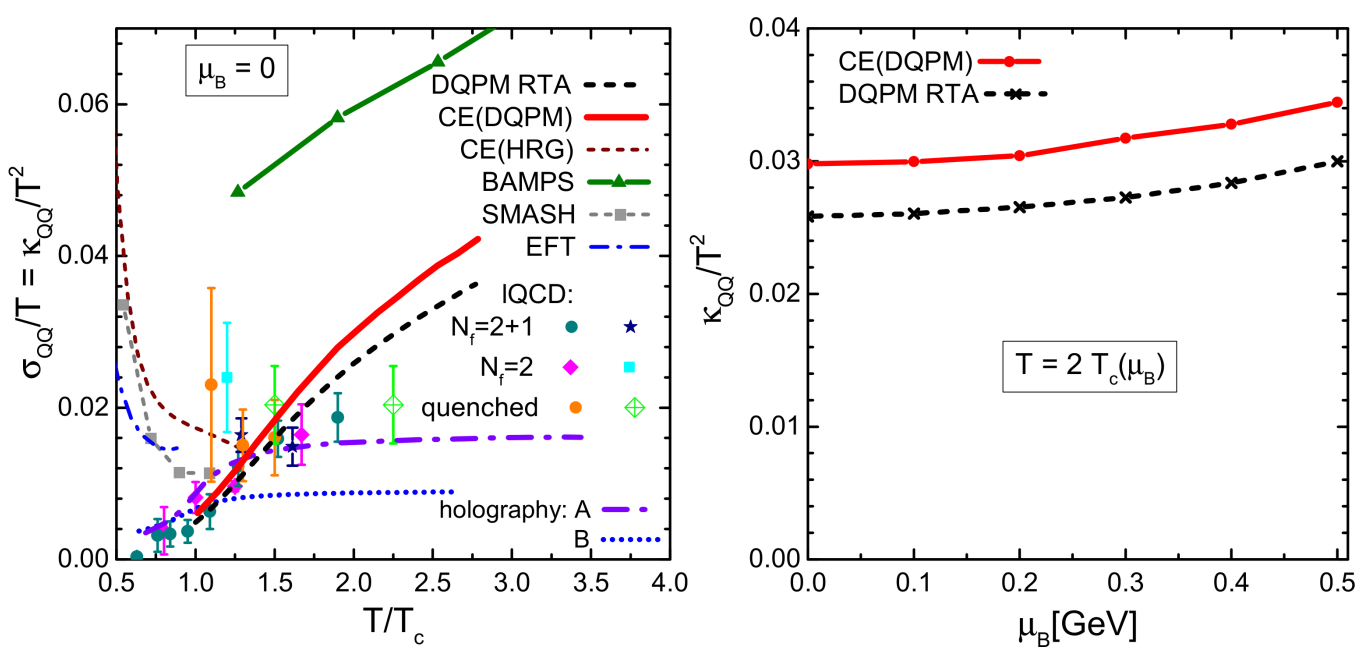

Figure 2.14: Left: Scaled electric conductivity, $\sigma_{\mathrm{QQ}} / T=\kappa_{\mathrm{QQ}} / T^{2}$, as a function of the scaled temperature $T / T_{c}$ at vanishing chemical potentials, $\mu_{q}=0$, from various approaches. The results from the CE (DQPM) are shown by the red solid line, and from DQPM RTA by the black dashed line. These are compared to results from the lattice QCD calculations: quenched: orange circle-shaped points [109], light green rhombus-shaped points [110], $N_{f}=2$ : light cyan circle-shaped points [94], magenta rhombus-shaped points [95], and $N_{f}=2+1$ : dark cyan circle-shaped points [96] and blue stars [111]), the kinetic partonic cascade model BAMPS (dark-green solid line with triangular-shaped points) [112], and from non-conformal EMT holographic models A [113] (violet dashed-dotted line) and B [49] (blue dotted line). For $T<T_{c}=0.158 \mathrm{GeV}$ we show results from a variety of hadronic models: SMASH $[40,105,114]$ (grey short-dashed line with squared points), effective field theory (EFT) [115] (blue dashed-dotted line), and CE tuned to a hadron gas [CE (HRG)] from Refs. [52, 104, 106] (dark-red dashed line). Right: Scaled electric conductivity of the QGP at fixed scaled temperature, $T=2 T_{c}\left(\mu_{\mathrm{B}}\right)$, and $\mu_{\mathrm{Q}}=\mu_{\mathrm{S}}=0$ are shown for varying baryon chemical potential $\mu_{\mathrm{B}}$ from the DQPM RTA (black dashed line with cross-shaped points) and the CE (DQPM) (red solid line with circle-shaped points) evaluation. The figure is adopted from [6].

We note that the DQPM RTA and CE (DQPM) results agree with the lattice QCD results near the transition region, $1 \leq T / T_{c} \leq 1.5$. We again point out the obvious quadratic dependence on temperature, which was briefly justified above. Given the limitations of the Chapman-Enskog and RTA methods discussed above, we expect that a realistic result for the conductivities might lie between the DQPM RTA and CE (DQPM) evaluations.

Moreover in Fig. 2.14 we show estimates from hadronic models: the hadronic transport model SMASH [40,105, 114] (grey short-dashed line with squared points), effective field theory (EFT) [115] (blue dashed-dotted line), and CE for a hadron gas CE (HRG) from Refs. [52, 104, 106] (dark-red dashed line). However, results from the hadronic models substantially overestimate the 1QCD data in the vicinity of $T_{c}$ as well as the results from the non-conformal holographic models A [117] (blue dotted line) and B [113] (violet dasheddotted line. The DQPM RTA results are in a good agreement in the vicinity of the phase transition with the previous estimations for DQPM* from Ref. [47], where a non-relativistic formula for the electric conductivity was used, which results in the linear dependence of the 



Figure 2.15: Scaled diffusion coefficients $\kappa_{q q^{\prime}} / T^{2}$ from the DQPM as a function of $T$ and $\mu_{B}$ obtained within the RTA method from Eq. (180) for moderate chemical potentials $\mu_{B} \leq 0.5 \mathrm{GeV}$.

$\sigma_{Q Q} / T$ on temperature, while the presented DQPM results show the quadratic dependence near the phase transition.

Furthermore, in Fig. 2.14 we show the cross-electric conductivities, $\sigma_{\mathrm{BQ}}$ and $\sigma_{\mathrm{QS}}$, from the CE (DQPM) and the DQPM RTA calculation together with results achieved within 

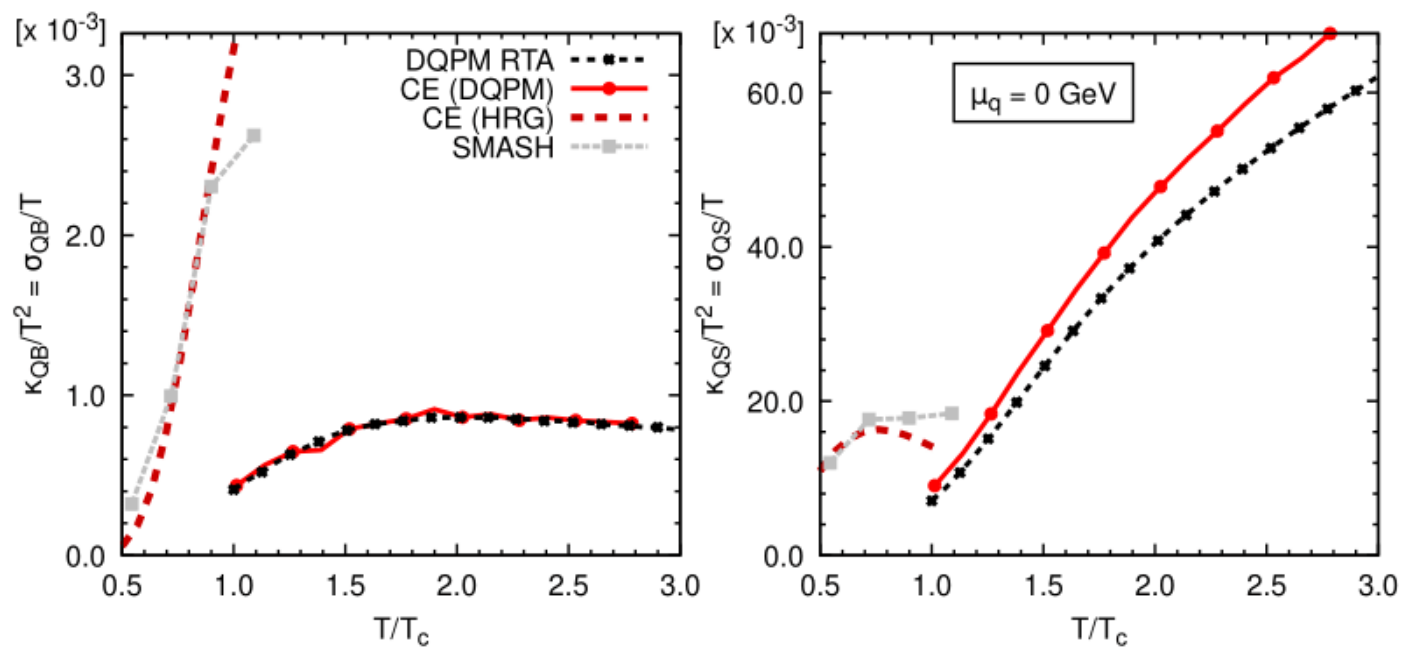

Figure 2.16: Scaled cross-electric conductivities, $\sigma_{\mathrm{QB}} / T^{2}$ (left) and $\sigma_{\mathrm{QS}} / T^{2}$ (right) as a function of scaled temperature $T / T_{c}$ at vanishing chemical potentials, $\mu_{q}=0$. We compare results from SMASH [40,105] (grey short-dashed line with square-shaped points), the DQPM RTA (black dashed line with cross-shaped points), and the CE (DQPM) (red solid line with circle-shaped points) and CE (HRG) [52,104] (dark-red dashed line). The figure is taken from [6].

SMASH [105] and the CE (HRG) evaluation from Refs. [52,104] for the same thermal considerations for the hadronic phase. Comparing the results in both phases, we find a significant disagreement for $\sigma_{Q B}$ around the crossover temperature. Furthermore, we find such discrepancies to a smaller extend in the other electric conductivities and in the coefficients to follow. It was pointed out in Ref. [105] that such disagreement may hint to a difference in the chemical composition of the adjacent phases.

We continue with the results for the strange sector: $\kappa_{\mathrm{SS}}$ and $\kappa_{\mathrm{SB}}$. The coefficient $\kappa_{\mathrm{SQ}}$, or equivalently $\sigma_{\mathrm{SQ}}=\sigma_{\mathrm{QS}}$, was already discussed above. Fig. 2.18 shows $\kappa_{\mathrm{SS}}$ and $\kappa_{\mathrm{SB}}$ as a function of temperature at vanishing chemical potentials. Further, we show their $\mu_{\mathrm{B}}$-dependence in Fig. 2.19 in the range $\mu_{\mathrm{B}}=0$ to $0.5 \mathrm{GeV}$ at fixed scaled temperature, $T=2 T_{c}\left(\mu_{\mathrm{B}}\right)$ and for vanishing electric and strange chemical potential. We compare results from the DQPM RTA and CE (DQPM) computation to results from CE (HRG) [52,104], and to the estimates from the non-conformal holographic model [117].

We find that the baryon-strange diffusion coefficient is negative due to the definition of strangeness carried by the s-quark as has been already advocated in Ref. [52, 104]. We obtain an almost quadratic dependence in temperature again, and a rather strong dependency on $\mu_{\mathrm{B}}$. However, the results from DQPM RTA for $\kappa_{\mathrm{SB}}$ in Fig. 2.19 show a slightly different $\mu_{\mathrm{B}}$-behavior than the results from CE (DQPM) for $\mu_{\mathrm{B}} \geq 0.3 \mathrm{GeV}$. Furthermore, extrancting from Fig. 2.18, in the vicinity of the crossover region the results from CE (HRG) for the hadronic phase, and the calculation from the DQPM RTA and CE (DQPM) agree well. As for the other diffusion coefficients, the scaled strange diffusion coefficient from holography has a different temperature dependence and smaller values in the vicinity of the crossover phase transition. Fig. 2.20 (left) shows the temperature 

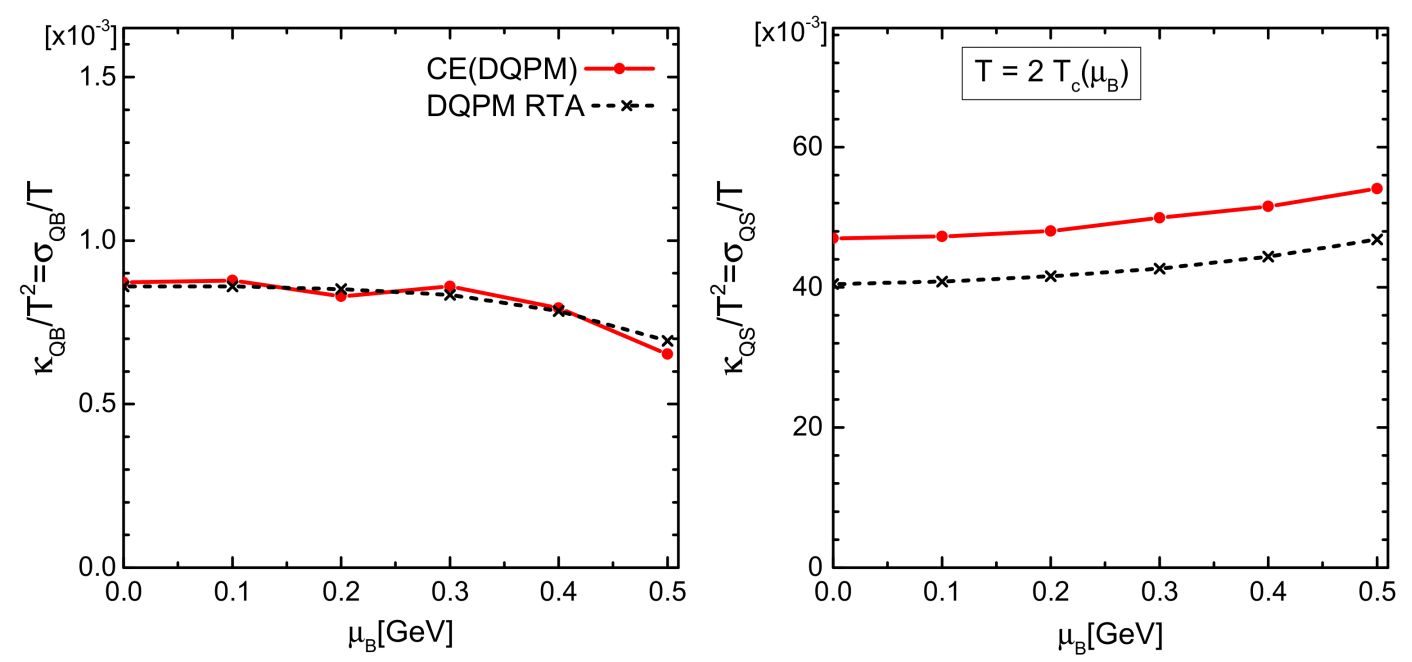

Figure 2.17: Scaled cross-electric conductivities, $\sigma_{\mathrm{QB}} / T^{2}$ (left) and $\sigma_{\mathrm{QS}} / T^{2}$ (right), from the DQPM RTA (black dashed line with cross-shaped points) and the CE (DQPM) evaluation at fixed scaled temperature, $T=2 T_{c}\left(\mu_{\mathrm{B}}\right)$, shown versus the baryon chemical potential $\mu_{\mathrm{B}}$ in the range 0 to $0.5 \mathrm{GeV}$. The other chemical potentials are fixed to zero, $\mu_{\mathrm{Q}}=0$ and $\mu_{\mathrm{S}}=0$. The figure is taken from [6].
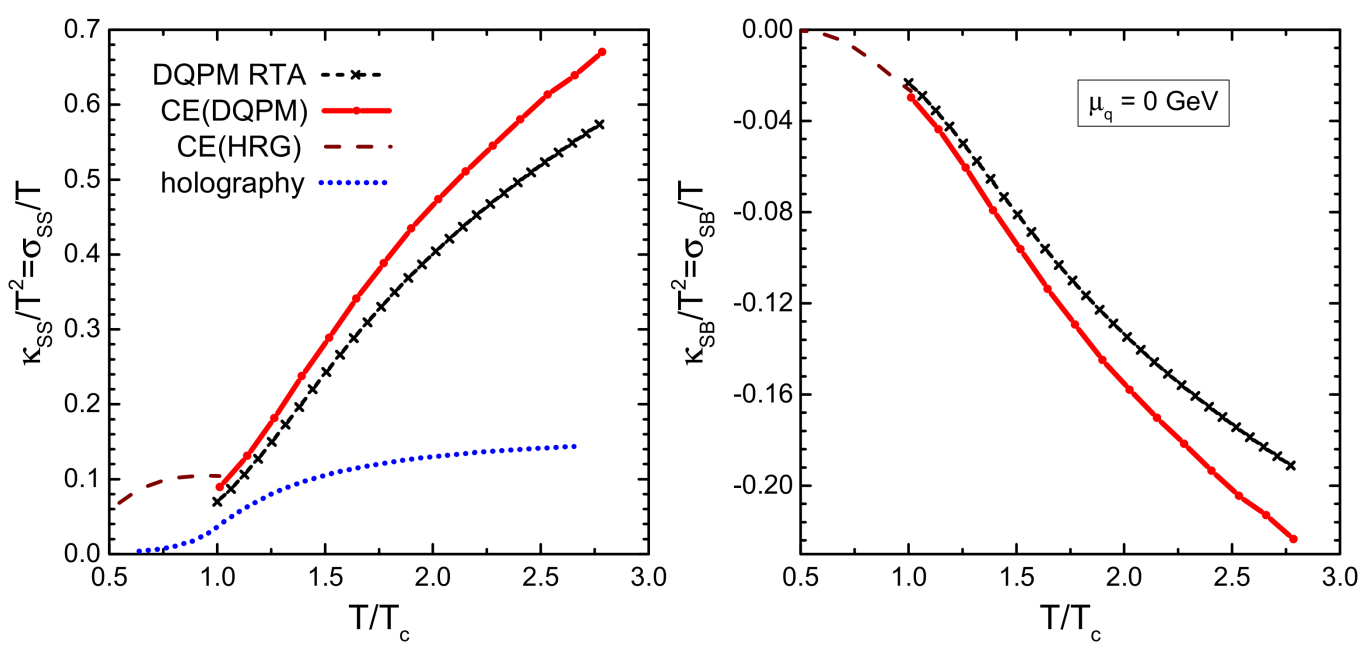

Figure 2.18: Scaled strange and strange-baryon diffusion coefficients, $\kappa_{\mathrm{SS}} / T^{2}$ (left) and $\kappa_{\mathrm{SB}} / T^{2}$ (right), as a function of scaled temperature $T / T_{c}$ at vanishing chemical potentials, $\mu_{q}=0$. We compare results from CE (DQPM) (red solid line with circles), DQPM RTA (black dashed-line with crossed-shaped points), the CE (HRG) $[52,104]$ (dark-red dashed line) and from conformal holography [117] (blue dotted line). The figure is taken from [6]. 

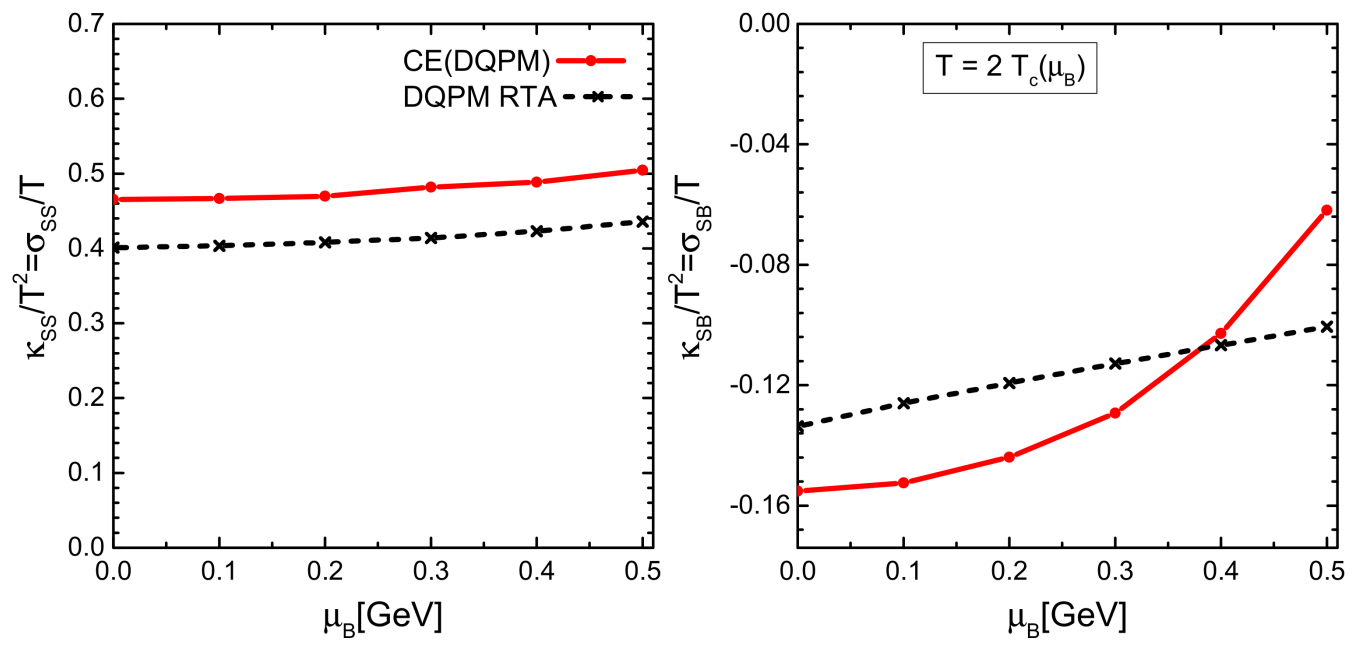

Figure 2.19: Scaled strange and strange-baryon diffusion coefficients, $\kappa_{\mathrm{SS}} / T^{2}$ (left) and $\kappa_{\mathrm{SB}} / T^{2}$ (right), from the DQPM RTA (black dashed line with cross-shaped points) and the CE (DQPM) evaluation at fixed scaled temperature, $T=2 T_{c}\left(\mu_{\mathrm{B}}\right)$, shown over baryon chemical potential $\mu_{\mathrm{B}}$ in range 0 to $0.5 \mathrm{GeV}$. Further, the other chemical potentials are fixed to zero, $\mu_{\mathrm{Q}}=0$ and $\mu_{\mathrm{S}}=0$. The figure is taken from [6].
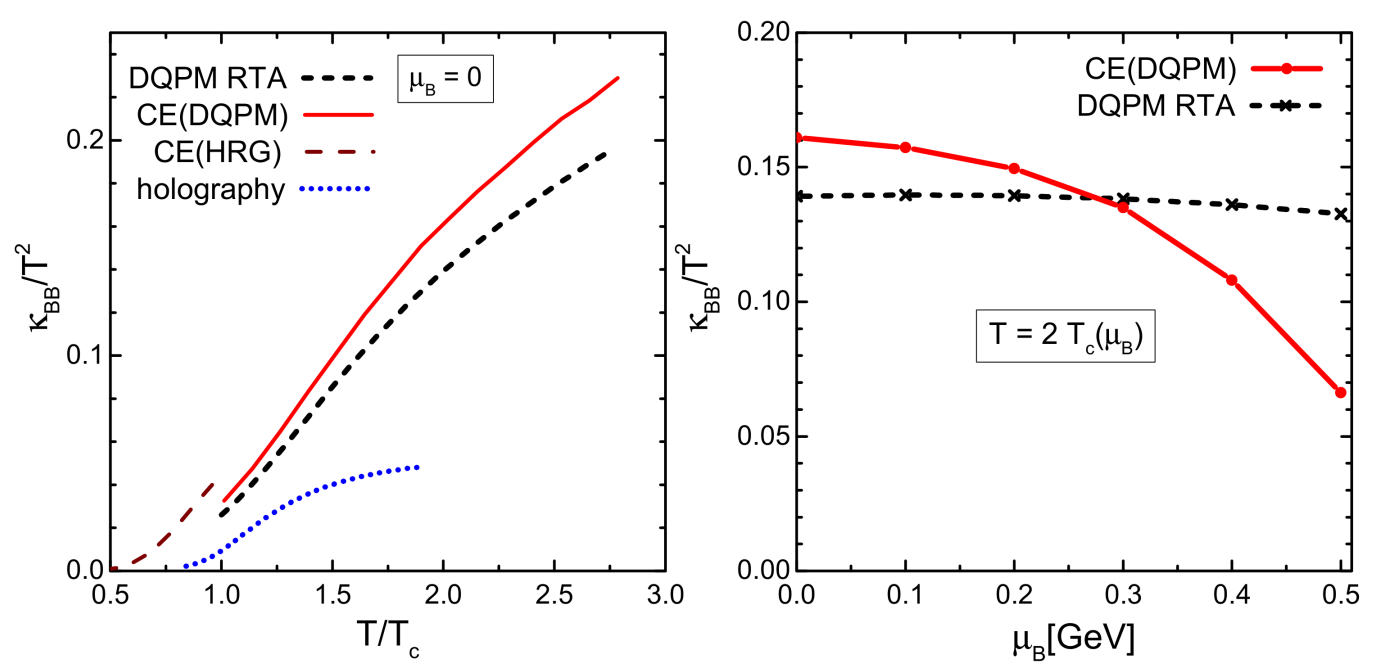

Figure 2.20: Scaled baryon diffusion coefficient, $\kappa_{\mathrm{BB}} / T^{2}$, (left) at vanishing chemical potentials, $\mu_{q}=\mu_{\mathrm{B}}=0$, as a function of the scaled temperature $T / T_{c}$ from various approaches and (right) at fixed scaled temperature, $T=2 T_{c}\left(\mu_{\mathrm{B}}\right)$, as a function of baryon chemical potential $\mu_{\mathrm{B}}$. The strange and electric potential are fixed to zero: $\mu_{\mathrm{S}}=0$ and $\mu_{\mathrm{Q}}=0$. We show results from the CE evaluation tuned to DQPM (red solid line), as described above, and tuned to a hadron gas from Refs. [52,104] (dark-red dashed line). We again compare to the calculation from DQPM RTA [2] (black dashed line with crosses) and to the non-conformal holographic model results [117] (blue dotted line) as for the electric conductivity. The figure is taken from [6]. 
dependence of the baryon diffusion coefficient for the QGP from the CE (DQPM) (red solid line) and DQPM RTA approaches (black dashed line with crosses). We also show the results from the non-conformal holographic model [117] (blue dotted lines). For temperatures below $T_{c}$ we present the CE (HRG) calculation from Refs. [52,104] (dark-red dashed line). The comparison is presented at zero chemical potentials $\mu_{q}=0$. Near $T_{c}$, the results of DQPM RTA, CE (DQPM), and CE (HRG) agree quite well with each other. Moreover, we show the dependence of $\mu_{\mathrm{B}}$ at fixed scaled temperature $T=2 T_{c}\left(\mu_{\mathrm{B}}\right)$ in Fig. 2.20 (right). The DQPM RTA shows a rather weak $\mu_{\mathrm{B}}$-dependence, while $\kappa_{\mathrm{BB}}$ from the CE (DQPM) decreases with $\mu_{\mathrm{B}}$. 


\subsection{Summary and Conclusions to Section 2}

- In this Chapter we recalled basic ingredients of the DQPM: model parameters, basic thermodynamic observables in comparison to the lQCD data and quasi-elastic scatterings between the partons.

- Transport coefficients of the QGP have been evaluated on the basis of the RTA, where we have evaluated the relaxation times using the parton interaction rates $\tau_{i}(\mathbf{p}, T, \mu)=\frac{1}{\Gamma_{i}(\mathbf{p}, T, \mu)}$, where all the quasi-elastic two-body scatterings are employed. Furthermore, we considered the spectral widths for the relaxation time $\tilde{\tau}_{i}\left(T, \mu_{B}\right)=\frac{1}{2 \gamma_{i}\left(T, \mu_{B}\right)}$ in order to check the consistency of the model parametrization. The scaled temperature $T / T_{c}$ and baryon chemical potential $\mu_{B}$ dependencies of transport coefficients such as shear and bulk viscosity, electric conductivity and baryon diffusion coefficient have been demonstrated. We find a good agreement between the two cases for all considered transport coefficients.

- We find only a rather weak dependence of the transport coefficients on $\mu_{B}$ compared to their values at $\mu_{B}=0$. The shear and bulk viscosities, and electric conductivities of the QGP increase slightly with increasing $\mu_{B}$ while the baryon-diffusion coefficient decreases. The latter has important consequences for the transport results for HICs observables, e.g., the baryon diffusion might enhance the difference between the proton and antiproton elliptic flows $v_{2}\left(p_{T}\right)$ and between the mean transverse momenta.

- Furthermore, a comparison between the RTA evaluations and the Chapman-Enskog method has been presented. We find a good agreement with the available predictions from the literature for the partonic phase for both methods, in particular the results for the scaled electrical conductivity are remarkably close to the 1QCD estimates at $\mu_{\mathrm{B}}=0$, as well as to the results for the hadronic phase. Interestingly, the nondiagonal diffusion coefficient $\kappa_{Q B}$ within the both methods does not agree well with the estimates for the hadronic phase near the phase transition. This variance can be interpreted as an indication of a difference in the chemical composition of the adjacent phases. In the literature there are several model calculations for diagonal conductivities (mostly $\kappa_{\mathrm{QQ}}$ ) that are similar to the results from the RTA approach but use numerous restrictive assumptions for the evaluation of relaxation times or cross sections. 


\section{3}

\section{Dynamical evolution of QCD matter from transport simulations}

In this Chapter we continue the exploration of the transport properties of QCD matter produced in HICs within a microscopic transport approach, which provides the full time evolution of the system. The evolution of the deconfined QCD phase has been also successfully described within hydrodynamic simulations and hybrid methods $[41,118-120]$. Nevertheless, to achieve a proper non-equilibrium description of the entire dynamics through possibly different phases up to the final asymptotic hadronic states, a microscopic treatment is needed. The Parton-Hadron-String Dynamics (PHSD) transport approach [43,62,121-123] is an off-shell transport approach based on the Kadanoff-Baym equations in first-order gradient expansion which allows for simulations of both the hadronic and the partonic phases. This approach sequentially describes the full evolution of relativistic heavy-ion collisions from the initial hard collisions and formation of strings, the deconfinement with a dynamic phase transition to a strongly interacting QGP, to hadronization and subsequent interactions in the expanding hadronic phase.

The hadronic part is essentially equivalent to the conventional Hadron-String-Dynamics (HSD) approach [38], while the partonic dynamics is based on the dynamical quasi-particle model (DQPM) described in the previous Chapter. It was found that the PHSD approach well describes observables from $\mathrm{p}+\mathrm{A}$ and $\mathrm{A}+\mathrm{A}$ collisions from SPS to LHC energies including electromagnetic probes such as photons and dileptons [62].

In order to tackle the new challenge - evolution of the partonic systems at higher $\mu_{B}$ - the PHSD approach has been recently extended to incorporate partonic quasiparticles and their differential cross sections that depend not only on temperature $T$ as in the previous PHSD studies, but also on chemical potential $\mu_{B}$ explicitly. Within this extended approach, the 'bulk' observables in HICs for different energies - from AGS to RHIC, - for symmetric $\mathrm{Au}+\mathrm{Au} / \mathrm{Pb}+\mathrm{Pb}$ collisions have been studied. Only a small influence of $\mu_{B}$ dependencies of the parton properties (masses and widths) and their interaction cross sections has been found in bulk observables.

Furthermore, we extend our study to more sensitive observables, such as collective flow coefficients and a manifestation of the $\mu_{B}$ dependencies of partonic cross sections in the flow coefficients. In addition, we explore the relations between the in and out-of equilibrium QGP by means of transport coefficients and collective flows.

Part of the results presented below have been included in publications $[1,4,7,124] .{ }^{2}$

$2 \quad$ This work was done in collaboration with Pierre Moreau (Goethe U.), who implemented new partonic cross sections in the PHSD5.0. The development of programs for the evaluation of the flow coefficients, modifications of the partonic mean-field potentials, calculations of the flow coefficients, the rapidity and $p_{T}$ distributions in symmetric heavy-ion collisions, as well as plotting routines have been realized by the author of the present thesis. 


\subsection{Generalized transport equations from the Kadanoff-Baym equations}

An effective method for studying systems far from equilibrium is the closed-time or the Schwinger-Keldysh formalism [125, 126] for Green's functions. The equations of motion for Green's functions can be interpreted as a quantum generalization of the Boltzmann equation. Let us consider for simplicity a scalar field theory. The two-point Green's function in the out-of-equilibrium field theory can be defined on a closed time contour (CTC) or the Shwinger-Keldysh contour as:

$$
G_{C}(x, y)=-i\left\langle T_{C}\left[\phi(x) \phi^{+}(y)\right]\right\rangle
$$

Here $T_{c}$ denotes the time ordering along the contour $C$, which is depicted in Fig. 3.1. This path begins at some $t_{0}$, goes to some time $t_{m} \rightarrow+\infty$, and then returns to $t_{0}$. The contour has two branches: on the branch $C^{+}$the time-ordering is chronological, while on the branch $C^{-}$it is anti-chronological.

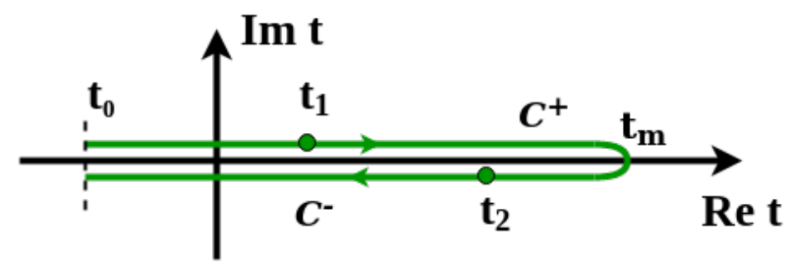

Figure 3.1: Closed time contour $C$ in the Schwinger-Keldysh formalism. $C^{ \pm}$denote the time-ordered branch and the anti-time-ordered branch, respectively.

Self-consistent equations of motion for these Green's functions can be obtained within the two-particle irreducible $(2 P I)$ effective action $\Gamma[G][127]$. Using functional variation of $\Phi[G]$ one can determine the self-energy by

$$
\Sigma_{C}(x, y)=2 i \frac{\delta \Phi}{\delta G(x, y)}
$$

Here $\Phi[G]$ represents the sum of all closed $2 P I$ diagrams built up by full propagators $G$. The equations of motion for the Green's function follows from the stationarity condition:

$$
\frac{\delta \Gamma}{\delta G(x, y)}=0
$$

The two-point function $G_{C}(x, y)$ satisfies the contour Dyson-Schwinger equation:

$$
G_{C}(x, y)=G_{C}^{0}(x, y)+\int_{C} d x^{\prime} \int_{C} d x^{\prime \prime} G_{C}^{0}\left(\left(x, x^{\prime}\right) \Sigma_{C}\left(x^{\prime}, x^{\prime \prime}\right) G_{C}\left(x^{\prime \prime}, y\right),\right.
$$

Here $G_{C}^{0}$ is the free (tree level) propagator. The formulation of the contour time ordering can be defined in terms of the usual time-ordered Green's functions along the real axis: the causal (time-ordered), the anticausal, the lesser $(<)$ and the greater $(>$ ) which read [128-130] 


$$
\begin{aligned}
i G^{c}(x, y) & =i G^{++}(x, y)=T^{c}\left[\hat{\phi}(x) \hat{\phi}^{\dagger}(y)\right] \\
i G^{<}(x, y) & =i G^{+-}(x, y)=\hat{\phi}^{\dagger}(y) \hat{\phi}(x) \\
i G^{>}(x, y) & =i G^{-+}(x, y)=\hat{\phi}(x) \hat{\phi}^{\dagger}(y) \\
i G^{a}(x, y) & =i G^{--}(x, y)=T^{a}\left[\hat{\phi}(x) \hat{\phi}^{\dagger}(y)\right] .
\end{aligned}
$$

Here $T^{c}$ and $T^{a}$ are, respectively, the causal and anticausal time-ordering operators. $G^{\lessgtr}$ are also known as the Wightmann functions. In Eqs. (81-84) the index \pm corresponds to the branch $C^{ \pm}$where the time argument of $x$ and $y$ are situated on the closed time contour, shown in Fig. 3.1. It is convenient to use the matrix representation of Green's function in Eq. (77):

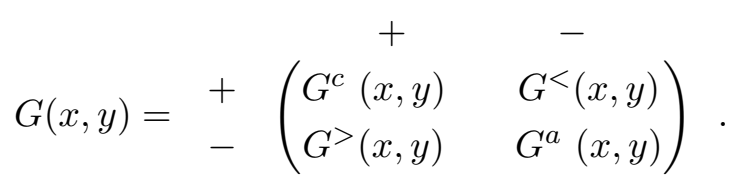

However, the four functions are not independent. The causal and anticausal functions can be expressed through $G^{\lessgtr}$ as

$$
G^{c / a}(x, y)=\theta\left[ \pm\left(x^{0}-y^{0}\right)\right] G^{>}(x, y)+\theta\left[ \pm\left(y^{0}-x^{0}\right)\right] G^{<}(x, y)
$$

Here the $\theta$-function is the usual Heaviside step function. The retarded and advanced Green's functions, which can be useful to calculate a physical response like transport coefficients, can be defined as

$$
G^{R / A}(x, y)= \pm \theta\left[ \pm\left(x^{0}-y^{0}\right)\right]\left(G^{>}(x, y)-G^{<}(x, y)\right)= \pm \theta\left[ \pm\left(x^{0}-y^{0}\right)\right] A(x, y)
$$

Here $A$ denotes the spectral function, however, it is more conveniently used in momentum space since it contains information about spectral properties of states.

Finally, the time evolution of the Wightman functions can be described via a set of Kadanoff-Baym equations [131]:

$$
\begin{aligned}
\left(-\partial_{x}^{\mu} \partial_{\mu}^{x}-m^{2}-\Sigma^{\delta}(x)\right) G^{\lessgtr}(x, y) & =\int_{t_{0}}^{x^{0}} d z^{0} \int d^{3} z\left(\Sigma^{>}(x, z)-\Sigma^{<}(x, z)\right) G^{\lessgtr}(z, y) \\
& -\int_{t_{0}}^{y^{0}} d z^{0} \int d^{3} z \Sigma^{\lessgtr}(x, z) \quad\left(G^{>}(z, y)-G^{<}(z, y)\right),
\end{aligned}
$$




$$
\begin{aligned}
\left(-\partial_{y}^{\mu} \partial_{\mu}^{y}-m^{2}-\Sigma^{\delta}(y)\right) G^{\lessgtr}(x, y) & =\int_{t_{0}}^{x^{0}} d z^{0} \int d^{3} z\left(G^{>}(x, z)-G^{<}(x, z)\right) \Sigma^{\lessgtr}(z, y) \\
& -\int_{t_{0}}^{y^{0}} d z^{0} \int d^{3} z G^{\lessgtr}(x, z) \quad\left(\Sigma^{>}(z, y)-\Sigma^{<}(z, y)\right) .
\end{aligned}
$$

Here the contour-ordered self-energy has been separated into a local $\Sigma^{\delta}$ and non-local parts, which can be written, as a sum of contour $\theta$ functions. These equations can be simplified by employing of retarded (advanced) Green's functions $G^{R / A}$ defined in Eq. (87) and retarded(advanced) self-energies $\Sigma^{R / A}$ as

$$
\begin{gathered}
\left(-\partial_{x}^{\mu} \partial_{\mu}^{x}-m^{2}-\Sigma^{\delta}(x)\right) G^{\lessgtr}(x, y)=\int_{t_{0}}^{\infty} d z^{0} \int d^{3} z\left(\Sigma^{R}(x, z) G^{\lessgtr}(z, y)+\Sigma^{\lessgtr}(x, z) G^{A}(z, y)\right) \\
\left(-\partial_{y}^{\mu} \partial_{\mu}^{y}-m^{2}-\Sigma^{\delta}(y)\right) G^{\lessgtr}(x, y)=\int_{t_{0}}^{\infty} d z^{0} \int d^{3} z\left(G^{R}(x, z) \Sigma^{\lessgtr}(z, y)+G^{\lessgtr}(x, z) \Sigma^{A}(z, y)\right) .
\end{gathered}
$$

Alternatively, equations of motion for the retarded (advanced) Green's functions can be obtained from the Schwinger-Dyson equation (80):

$$
\begin{aligned}
& \left(-\partial_{x}^{\mu} \partial_{\mu}^{x}-m^{2}-\Sigma^{\delta}(x)\right) G^{R / A}(x, y)=\delta^{(4)}(x, y)+\int d^{4} z \Sigma^{R / A}(x, z) G^{R / A}(z, y) \\
& \left(-\partial_{y}^{\mu} \partial_{\mu}^{y}-m^{2}-\Sigma^{\delta}(y)\right) G^{R / A}(x, y)=\delta^{(4)}(x, y)+\int d^{4} z G^{R / A}(x, z) \Sigma^{R / A}(z, y) .
\end{aligned}
$$

Equations (91) depend on retarded (advanced) quantities and where the integration extends to the full space and time.

From the Kadanoff-Baym equations the off-shell transport equations can be derived. To aim this goal, a Wigner transformation is performed with respect to the relative coordinate $\Delta x=x-y:$

$$
\bar{G}(p, X)=\int d^{4} \Delta x e^{i \Delta x_{\mu} p^{\mu}} G(X+\Delta x / 2, X-\Delta x / 2) .
$$

Here $X=\frac{x+y}{2}$ denotes the central coordinate, while $p=(\omega, \mathbf{p})$ is the four-momentum. The Wigner transformed Green's functions $\bar{G}(\mathbf{r}, \mathbf{p}, \omega, t)$ depend on the coordinate $\mathbf{r}$, momentum $\mathbf{p}$, energy $\omega$ and time $t$. It is important to note that the right hand-side of the Kadanoff-Baym equations involve a convolution, which is hard to transform in all orders. In transport theory it is assumed that all functions are only smoothly varying with their mean space-time coordinates and thus one may consider only first-order derivatives, while all terms proportional to the second and higher derivatives of the mean space-time coordinates will be neglected. Therefore, to perform the Wigner transformation of the right hand-side one can employ the first-order gradient approximation, i.e. approximating the exponential function in the Fourier transformation to first order.

Furthermore, applying the Botermans-Malfliet scheme [132], the generalized transport equation can be written as $[38,122]$ 


$$
\frac{1}{2} \bar{A} \bar{\Gamma}\left[\left\{\bar{M}, i G^{<}\right\}-\frac{1}{\bar{\Gamma}}\left\{\bar{\Gamma}, \bar{M} \cdot i G^{<}\right\}\right]=i \bar{\Sigma}^{<} i \bar{G}^{>}-\bar{\Sigma}^{>} i \bar{G}^{<}
$$

Here the relativistic Poisson bracket is used

$$
\{\bar{F}(P, X), \bar{G}(P, X)\}=\partial_{\mu}^{P} \bar{F}(P, X) \partial_{X}^{\mu} \bar{G}(P, X)-\partial_{X}^{\mu} \bar{F}(P, X) \partial_{\mu}^{P} \bar{G}(P, X)
$$

In Eq. (93) $\bar{M}$ is the mass function defined as

$$
\bar{M}=P_{0}^{2}-\mathbf{P}^{2}-m^{2}-\bar{\Sigma}^{\delta}-R e \bar{\Sigma}^{R}
$$

On the left hand-side of Eq. (93) one can recognize the drift term and the contribution of the real part of the self-energy, also known as the Vlasov term. Moreover, the real part of the self-energy $\operatorname{Re} \bar{\Sigma}^{R}$ acts as an effective potential, whose space-time derivative gives a force term. The term $\left\{\bar{\Gamma}, \bar{M} \cdot i G^{<}\right\}$vanishes in the on-shell limit and is responsible for the proper off-shell propagation. Equation (93) can be viewed as a quantum generalization of the Boltzmann equation and can describe off-shell effects, which play an important role for the strongly interacting systems. As shown in Refs. [133-135], the off-shell dynamics is important for hadronic resonances with moderate lifetime in the vacuum, but strongly reduced lifetime in the nuclear medium (such as $\omega$ and $\rho$ mesons). It also provides a correct description of dilepton decays of $\rho$ mesons with masses close to the two-pion decay threshold. A detailed discussion about the differences for the propagation of strongly interacting systems via the Boltzmann equation and generalized transport equation can be found in Ref. [135].

\subsection{Generalized test-particle representation}

In order to solve the generalized transport equation in consistent first-order gradient expansion, an extended test-particle Ansatz [136] can be employed. It approximates the (real and positive semi-definite) Green's function $i \bar{G}^{<}$as a sum of point-like particles:

$$
i \bar{G}^{<}\left(P_{0}, \mathbf{P}, t, \mathbf{X}\right) \sim \sum_{i=1}^{N} \frac{1}{2 P_{0}} \delta^{(3)}\left(\mathbf{X}-\mathbf{X}_{i}(t)\right) \delta^{(3)}\left(\mathbf{P}-\mathbf{P}_{i}(t)\right) \delta\left(P_{0}-\epsilon_{i}(t)\right)
$$

Here the position of the test-particle $i$ at time $t$ is denoted by $\mathbf{X}_{i}(t)$, and its 4-momentum by $\left(\epsilon_{i}(t), \mathbf{P}_{i}(t)\right)$. We first study the free propagation of particles in between collisions, i.e. by neglecting the collision terms $I_{\text {coll }}$ in the generalized transport equation (93), which we can rewrite in the form $[130,133]$ : 


$$
\begin{aligned}
2 P^{\mu} \partial_{\mu}^{X} i \bar{G}^{<} & -\partial_{\mu}^{X}\left[\left(\partial_{P}^{\mu} \operatorname{Re} \bar{\Sigma}^{R}+\frac{P^{2}-m^{2}-R e \bar{\Sigma}^{R}}{\bar{\Gamma}} \partial_{P}^{\mu} \bar{\Gamma}\right) i \bar{G}^{<}\right] \\
& +\partial_{P}^{\mu}\left[\left(\partial_{\mu}^{X} \operatorname{Re} \bar{\Sigma}^{R}+\frac{P^{2}-m^{2}-R e \bar{\Sigma}^{R}}{\bar{\Gamma}} \partial_{\mu}^{X} \bar{\Gamma}\right) i \bar{G}^{<}\right]=0,
\end{aligned}
$$

where the contribution of the self-energy $\bar{\Sigma}^{\delta}$ has been incorporated into $\operatorname{Re} \bar{\Sigma}^{R}$. By replacing $i \bar{G}^{<}$in the previous equation by the test-particle approximation (96), one can separate the contributions from similar coefficients of delta functions in order to obtain equations of motion for the test-particles as [122,133,137]:

$$
\begin{aligned}
\frac{d \mathbf{X}_{i}}{d t} & =\frac{1}{1-C_{(i)}} \frac{1}{2 \epsilon_{i}}\left[2 \mathbf{P}_{i}+\nabla_{\mathbf{P}_{i}} \operatorname{Re} \bar{\Sigma}_{(i)}^{R}+\frac{\epsilon_{i}^{2}-\mathbf{P}_{i}^{2}-m^{2}-\operatorname{Re} \bar{\Sigma}_{(i)}^{R}}{\bar{\Gamma}_{(i)}} \nabla_{\mathbf{P}_{i}} \bar{\Gamma}_{(i)}\right], \\
\frac{d \mathbf{P}_{i}}{d t} & =\frac{-1}{1-C_{(i)}} \frac{1}{2 \epsilon_{i}}\left[\nabla_{\mathbf{X}_{i}} \operatorname{Re} \bar{\Sigma}_{(i)}^{R}+\frac{\epsilon_{i}^{2}-\mathbf{P}_{i}^{2}-m^{2}-\operatorname{Re} \bar{\Sigma}_{(i)}^{R}}{\bar{\Gamma}_{(i)}} \nabla_{\mathbf{X}_{i}} \bar{\Gamma}_{(i)}\right] \\
\frac{d \epsilon_{i}}{d t} & =\frac{1}{1-C_{(i)}} \frac{1}{2 \epsilon_{i}}\left[\partial_{t} \operatorname{Re} \bar{\Sigma}_{(i)}^{R}+\frac{\epsilon_{i}^{2}-\mathbf{P}_{i}^{2}-m^{2}-\operatorname{Re} \bar{\Sigma}_{(i)}^{R}}{\bar{\Gamma}_{(i)}} \partial_{t} \bar{\Gamma}_{(i)}\right]
\end{aligned}
$$

The multiplication factor $C_{(i)}$ contains energy derivatives of the self-energy as

$$
C_{(i)}=\frac{1}{2 \epsilon_{i}}\left[\partial_{\epsilon_{i}} \operatorname{Re} \bar{\Sigma}_{(i)}^{R}+\frac{\epsilon_{i}^{2}-\mathbf{P}_{i}^{2}-m^{2}-\operatorname{Re} \bar{\Sigma}_{(i)}^{R}}{\bar{\Gamma}_{(i)}} \partial_{\epsilon_{i}} \bar{\Gamma}_{(i)}\right] .
$$

The multiplication factor gives a shift of the system time $t$ to the 'eigentime' of the particle $i$ defined by $\tilde{t}_{i}=t /\left(1-C_{(i)}\right)$. This correction factor is a generalization of the quasiparticle renormalization factor arising for particles with finite width [132]. Moreover, one can see that the evolution equations for $d \mathbf{X}_{i} / d \tilde{t}_{i}, d \mathbf{P}_{i} / d \tilde{t}_{i}$ and $d \epsilon_{i} / d \tilde{t}_{i}$, are the same as Eqs. (98)(99)-(100) without this normalization factor $1-C_{(i)}$. For a momentum independent width $\bar{\Gamma}_{(i)}(X)$, one can consider the independent variable $M^{2}=P^{2}-R e \bar{\Sigma}^{R}$ instead of the energy $P_{0}$, which is then fixed as:

$$
P_{0}^{2}=\mathbf{P}^{2}+M^{2}+\bar{\Sigma}^{\delta}+R e \bar{\Sigma}^{R}
$$

IN this case Eq. (100) can be written as an evolution equation for the functions $\Delta M_{i}^{2}$,

$$
\frac{d \Delta M_{i}^{2}}{d t}=\frac{\Delta M_{i}^{2}}{\bar{\Gamma}_{(i)}} \frac{d \bar{\Gamma}_{(i)}}{d t} \quad \leftrightarrow \quad \frac{d}{d t} \ln \left(\frac{\Delta M_{i}^{2}}{\bar{\Gamma}_{(i)}}\right)=0
$$

Here $\Delta M_{i}^{2}=M_{i}^{2}-m^{2}$ is a measure of the off-shellness of the particle $i$, whose evolution is found to be directly proportional to the ratio $\bar{M} / \bar{\Gamma}$. This ratio also appears in Eq. (97) as a weighting factor for the changes induced by the variation of the imaginary part of the 
self-energy $\bar{\Gamma}=-2 \operatorname{Im} \bar{\Sigma}^{R}$.

\subsection{Parton-Hadron-String Dynamics transport approach in a nutshell}

We start with recalling of the basic ideas of the PHSD transport approach. The PHSD transport approach $[43,62,121-123]$ is a microscopic off-shell transport approach for the description of strongly interacting hadronic and partonic matter in and out-of equilibrium. It is based on the solution of the generalized transport equations presented in the previous section, employing 'resummed' Green's functions from the DQPM $[45,46,56]$ for the partonic phase.

The DQPM, as described in the previous Chapter, has been introduced for the effective description of the QGP in terms of strongly interacting quarks and gluons with properties and interactions that are adjusted to reproduce 1 QCD results on the thermodynamics of the equilibrated QGP at finite temperature $T$ and baryon (or quark) chemical potential $\mu_{B}$. Masses and widths for the partons depend on temperature $T$ and baryon chemical potential $\mu_{B}$ are employed as input in the present PHSD calculations. These values for the masses and widths have been fixed by fitting the lattice QCD results from Ref. $[22,60]$ in thermodynamic equilibrium. One can see that the masses of quarks and gluons decrease with increasing $\mu_{B}$, and a similar trend holds for the widths of partons. Moreover, the DQPM also allows to define a scalar mean-field $U_{s}\left(\rho_{s}\right)$ for quarks and antiquarks which can be expressed by the derivative of the potential energy density with respect to the scalar density $\rho_{s}$

$$
U_{s}\left(\rho_{s}\right)=\frac{d V_{p}\left(\rho_{s}\right)}{d \rho_{s}}
$$

which is evaluated numerically. Here, the potential energy density is defined by

$$
V_{p}\left(T, \mu_{q}\right)=T_{g-}^{00}\left(T, \mu_{q}\right)+T_{q-}^{00}\left(T, \mu_{q}\right)+T_{\bar{q}-}^{00}\left(T, \mu_{q}\right),
$$

where the different contributions $T_{j-}^{00}$ correspond to the space-like part of the energymomentum tensor component $T_{j}^{00}$ of parton $j=g, q, \bar{q}$ (cf. Section 3 in Ref. [45]). The scalar mean-field $U_{s}\left(\rho_{s}\right)$ for quarks and antiquarks is repulsive as a function of the parton scalar density $\rho_{s}$ and shows that the scalar mean-field potential is in the order of a few $\mathrm{GeV}$ for $\rho_{s}>10 \mathrm{fm}^{-3}$. The mean-field potential (104) is employed in the PHSD transport calculations and determines the force on a partonic quasiparticle $j$ which is proportional to

$$
M_{j} / E_{j} \nabla U_{s}(x)=M_{j} / E_{j} d U_{s} / d \rho_{s} \nabla \rho_{s}(x),
$$

where the scalar density $\rho_{s}(x)$ is determined numerically on a space-time grid.

Furthermore, a two-body interaction strength can be extracted from the DQPM as well from the quasiparticle width in line with Ref. [46]. For the QGP phase we consider the following elastic and inelastic interactions, which are included in the latest version of 
PHSD (v. 5.0):

$$
q q \leftrightarrow q q, \bar{q} \bar{q} \leftrightarrow \bar{q} \bar{q}, g g \leftrightarrow g g, g g \leftrightarrow g, q \bar{q} \leftrightarrow g, q g \leftrightarrow q g, g \bar{q} \leftrightarrow g \bar{q}
$$

exploiting 'detailed-balance' with cross sections calculated from the leading order Feynman diagrams employing the effective propagators and couplings $g^{2}\left(T / T_{c}\right)$ from the DQPM [1]. In Ref. [1], the differential and total off-shell cross sections have been evaluated as a function of the invariant energy of colliding off-shell partons $\sqrt{s}$ for each $T, \mu_{B}$. In the preceding studies within the PHSD framework (using v. 4.0 and below) the parton cross sections depend only on $T$ as evaluated in Ref. [138].

To employ the differential cross sections and parton masses into the PHSD5.0 approach, one has to determine $T$ and $\mu_{B}$ in each computational cell in space-time. This has been realised by implementing the Taylor expansion of the $1 \mathrm{QCD}$ equation-of-state and a diagonalization of the energy-momentum tensor from PHSD [1], which will be discussed in the next Section.

The transition from the partonic to the hadronic phase (and vice versa) is described by covariant transition rates for the fusion of quark-antiquark pairs or three quarks (antiquarks), respectively, obeying flavor current-conservation, color neutrality as well as energy-momentum conservation [43]. In particular, transition rates for $q+\bar{q}$ fusion to a mesonic state $m$ of four-momentum $p=(\omega, \mathbf{p})$ at space-time point $x=(t, \mathbf{x})$ reads

$$
\begin{aligned}
& \frac{d N_{m}(x, p)}{d^{4} x d^{4} p}=\operatorname{Tr}_{q} \operatorname{Tr}_{\bar{q}} \delta^{4}\left(p-p_{q}-p_{\bar{q}}\right) \delta^{4}\left(\frac{x_{q}+x_{\bar{q}}}{2}-x\right) \omega_{q} \rho_{q}\left(p_{q}\right) \omega_{\bar{q}} \rho_{\bar{q}}\left(p_{\bar{q}}\right) \\
& \quad \times\left|v_{q \bar{q}}\right|^{2} W_{m}\left(x_{q}-x_{\bar{q}},\left(p_{q}-p_{\bar{q}}\right) / 2\right) N_{q}\left(x_{q}, p_{q}\right) N_{\bar{q}}\left(x_{\bar{q}}, p_{\bar{q}}\right) \delta \text { (flavor, color). }
\end{aligned}
$$

Here we have introduced the shorthand notation,

$$
T r_{i}=\sum_{i} \int d^{4} x_{i} \int \frac{d^{4} p_{i}}{(2 \pi)^{4}}
$$

where $\sum_{i}$ denotes a summation over discrete quantum numbers (spin, flavor, color). $N_{i}(x, p)$ is the phase-space density of parton $i$ at space-time position $x$ and four-momentum $p$. In Eq. (107) $\delta$ (flavor, color) stands symbolically for the conservation of flavor quantum numbers as well as color neutrality of the formed hadronic state $m$ which can be viewed as a color-dipole or 'pre-hadron'. Since close to the phase transition the dynamical quarks and anti-quarks become very massive, the formed resonant 'prehadronic' color-dipole states $(q \bar{q}$ or $q q q)$ have high invariant mass, too, and sequentially decay to the ground state meson and baryon octets, thus increasing the total entropy. Furthermore, $v_{q \bar{q}}\left(\rho_{p}\right)$ is the effective quark-antiquark interaction from the DQPM (displayed in Fig. 10 of Ref. [45]) as a function of the local parton $(q+\bar{q}+g)$ density $\rho_{p}$ (or energy density). $W_{m}(x, p)$ denotes the dimensionless phase-space distribution of the formed 'pre-hadron'

$$
W_{m}\left(\xi, p_{\xi}\right)=\exp \left(\frac{\xi^{2}}{2 b^{2}}\right) \exp \left[2 b^{2}\left(p_{\xi}^{2}-\left(M_{q}-M_{\bar{q}}\right)^{2} / 4\right)\right]
$$


Here we use shorthand notations $\xi=x_{1}-x_{2}=x_{q}-x_{\bar{q}}$ and $p_{\xi}=\left(p_{1}-p_{2}\right) / 2=\left(p_{q}-p_{\bar{q}}\right) / 2$. The width parameter $b$ is defined as $\sqrt{\left\langle r^{2}\right\rangle}=b=0.66 \mathrm{fm}$ (in the rest frame) which corresponds to an average radius of mesons. $W_{m}(x, p)$ is Lorentz invariant by construction. In the limit of instantaneous hadron formation (i.e. $\xi^{0}=0$ ) it provides a Gaussian decrease in the relative distance squared $\left(\mathbf{r}_{1}-\mathbf{r}_{2}\right)^{2}$. The four-momentum dependence reads explicitly

$$
\left(E_{1}-E_{2}\right)^{2}-\left(\mathbf{p}_{1}-\mathbf{p}_{2}\right)^{2}-\left(M_{1}-M_{2}\right)^{2} \leq 0 .
$$

It results in a negative argument of the second exponential in Eq. (109) favoring the fusion of partons with low relative momenta $p_{q}-p_{\bar{q}}=p_{1}-p_{2}$.

The probability for a quark to hadronize is essentially proportional to the timestep $d t$ in the calculation, the number of possible hadronization partners in the volume $d V \sim 5 \mathrm{fm}^{3}$ and the transition matrix element squared (apart from the gaussian overlap function). For temperatures above $T_{c}$ the probability is rather small $(\ll 1)$ while for temperatures close to $T_{c}$ and below $T_{c}$ the matrix element becomes very large since it essentially scales with the effective coupling squared $g^{2}\left(T / T_{c}\right)$. Moreover, for a finite time-step $d t$ the probability becomes larger than 1 which implies that the quark has to hadronize with some of the potential antiquarks in the actual time-step if the temperature or energy density becomes too low. Furthermore, the gluons practically freeze out close to $T_{c}$ since the mass difference between quarks and gluons increases drastically with decreasing temperature and the reaction channel $g \leftrightarrow q+\bar{q}$ is close to equilibrium. This implies that all partons hadronize. On the hadronic side, PHSD includes explicitly the baryon octet and decouplet, the $0^{-}$and $1^{-}$-meson nonets as well as selected higher resonances as in the HSD approach [38]. In case of hadrons of higher masses $(>1.5 \mathrm{GeV}$ in case of baryons and $>1.3 \mathrm{GeV}$ in case of mesons) they are treated as 'strings' (color-dipoles), that decay to the known (low-mass) hadrons within the JETSET algorithm [139]. Note that PHSD and HSD merge at low energy density, in particular below the local critical energy density $\varepsilon_{c} \approx 0.5 \mathrm{GeV} / \mathrm{fm}^{3}$.

The initial conditions for the parton/hadron dynamical system have to be specified additionally. In order to describe relativistic heavy-ion reactions we start with two nuclei in their semi-classical groundstate, boosted towards each other with a velocity $\beta$ (in $z$ direction), fixed by the bombarding energy. The initial phase-space distributions of the projectile and target nuclei are determined in the local Thomas-Fermi limit as in the HSD transport approach [38] or the UrQMD model [36]. We recall that at relativistic energies the initial interactions of two nucleons are well described by the excitation of two color-neutral strings which decay in time to the known hadrons (mesons, baryons, antibaryons) [139]. Initial hard processes - i.e. the short-range high-momentum transfer reactions that can be well described by perturbative QCD - are treated in PHSD (as in HSD) via PYTHIA. The novel element in PHSD (relative to HSD) is the dissolution of 'pre-hadrons'. However, in PHSD the 'pre-hadrons' (or possibly formed hadrons) are only allowed to dissolve if the local energy density $\epsilon(x)$ (in the local rest frame) is above the transition energy density $\epsilon_{c}$ which in the DQPM is $\epsilon_{c} \approx 0.5 \mathrm{GeV} / \mathrm{fm}^{3}$. The mesonic strings then decay to 
quark-antiquark pairs according to an intrinsic quark momentum distribution,

$$
F(\mathbf{q}) \sim \exp \left(-2 b^{2} \mathbf{q}^{2}\right)
$$

in the meson rest-frame (cf. Eq. (109) for the inverse process). The parton final fourmomenta are selected randomly according to the momentum distribution (111), and the parton-energy distribution is fixed by the DQPM at given energy density $\epsilon\left(\rho_{s}\right)$ in the local cell with scalar parton density $\rho_{s}$. The flavor content of the $q \bar{q}$ pair is fully determined by the flavor content of the initial string. By construction the "string melting" to massive partons conserves energy and momentum as well as the flavor content. In contrast to Ref. [140] the partons are of finite mass - in line with their local spectral function - and obtain a random color $c=(1,2,3)$ or $(r, b, g)$ in addition. Of course, the color appointment is color neutral, i.e. when selecting a color $c$ for the quark randomly the color for the antiquark is fixed by $-c$. Similarly, the baryonic strings melt into a quark and a diquark while the diquark decays to two quarks. Dressed gluons are generated by the fusion of nearest neighbor $q+\bar{q}$ pairs $(q+\bar{q} \rightarrow g)$ that are flavor neutral until the ratio of gluons to quarks reaches the value $N_{g} /\left(N_{q}+N_{\bar{q}}\right)$ given by the DQPM for the energy density of the local cell. This recombination is performed for all cells in space during the passage time of the target and projectile (before the calculation continues with the next time step) and conserves the four-momentum as well as the flavor currents. We note, however, that the initial phase in PHSD is dominated by quark and anti-quark degrees of freedom [141]. Apart from proton-proton, proton-nucleus or nucleus-nucleus collisions the PHSD approach can also be employed to study the properties of the interacting hadron/parton system, such as transport coefficients, in a finite box with periodic boundary conditions [84]. To this aim the system is initialized by a homogeneous distribution of test-particles in a finite box with a momentum distribution close to a thermal one. 


\subsection{Modelling Heavy-Ion Collisions}

In order to solve transport equations in the PHSD framework test-particle Anzatz is employed for the drift term, while for the evaluation of a collisional integral the stochastic method is used in order to find colliding partners within the same time step. The parallel ensembles method is applied in the PHSD, where the simulations are performed for $N$ parallel ensembles, which have a similar number of particles. The collisions between the particles are allowed only inside the same ensemble, whereas the macroscopic quantities are averaged over the ensembles. In this Section we will investigate the sensitivity of 'bulk' observables such as rapidity and transverse momentum distributions as well as flow coefficients of different hadrons produced in heavy-ion collisions from AGS to top RHIC energies on the details of the QGP interactions and the properties of the partonic degrees of freedom. To this aim, we have considered the following three settings for the transport simulations:

- PHSD4.0: the masses and widths of quarks and gluons also depend only on $T$. The cross sections for partonic interactions depend only on $T$ as evaluated in the 'box' calculations in Ref. [138] in order to merge the QGP interaction rates from all possible partonic channels to the total temperature dependent widths of the DQPM propagator. This method has been used in the PHSD codes (v. 4.0 or below) for extended studies of many hadronic observables in $\mathrm{p}+\mathrm{A}$ and $\mathrm{A}+\mathrm{A}$ collisions at different energies $[43,62,121-123,142]$.

- PHSD5.0 - $\mu_{B}=0$ : the masses and widths of quarks and gluons depend only on $T$; however, the differential and total partonic cross sections are obtained by calculations of the leading order Feynman diagrams employing the effective propagators and couplings $g^{2}\left(T / T_{c}\right)$ from the DQPM at $\mu_{B}=0$ [1]. Thus, the cross sections depend explicitly on the invariant energy of the colliding partons $\sqrt{s}$ and on $T$. This is realized in the PHSD5.0 by keeping $\mu_{B}=0$.

- PHSD5.0 - $\mu_{B}$ : the masses and widths of quarks and gluons depend on $T$ and $\mu_{B}$ explicitly; the differential and total partonic cross sections are obtained by calculations of the leading order Feynman diagrams from the DQPM and explicitly depend on the invariant energy $\sqrt{s}$, temperature $T$ and baryon chemical potential $\mu_{B}$. This is realized in the full version of PHSD5.0 [1].

\section{Extraction of $\left(T, \mu_{B}\right)$ in PHSD5.0}

In the transport approach, $T$ and $\mu_{B}$ are not natural observables, however, one can determine the energy density and baryon density in each cell. In order to extract $T$ and $\mu_{B}$ from the corresponding EoS of the partonic system one has to solve the following system of equations [1]:

$$
\left\{\begin{array}{l}
\epsilon^{\mathrm{EoS}}\left(T, \mu_{B}\right)=\epsilon^{\mathrm{PHSD}} / r(x) \\
n_{B}^{\mathrm{EoS}}\left(T, \mu_{B}\right)=n_{B}^{\mathrm{PHSD}} .
\end{array}\right.
$$




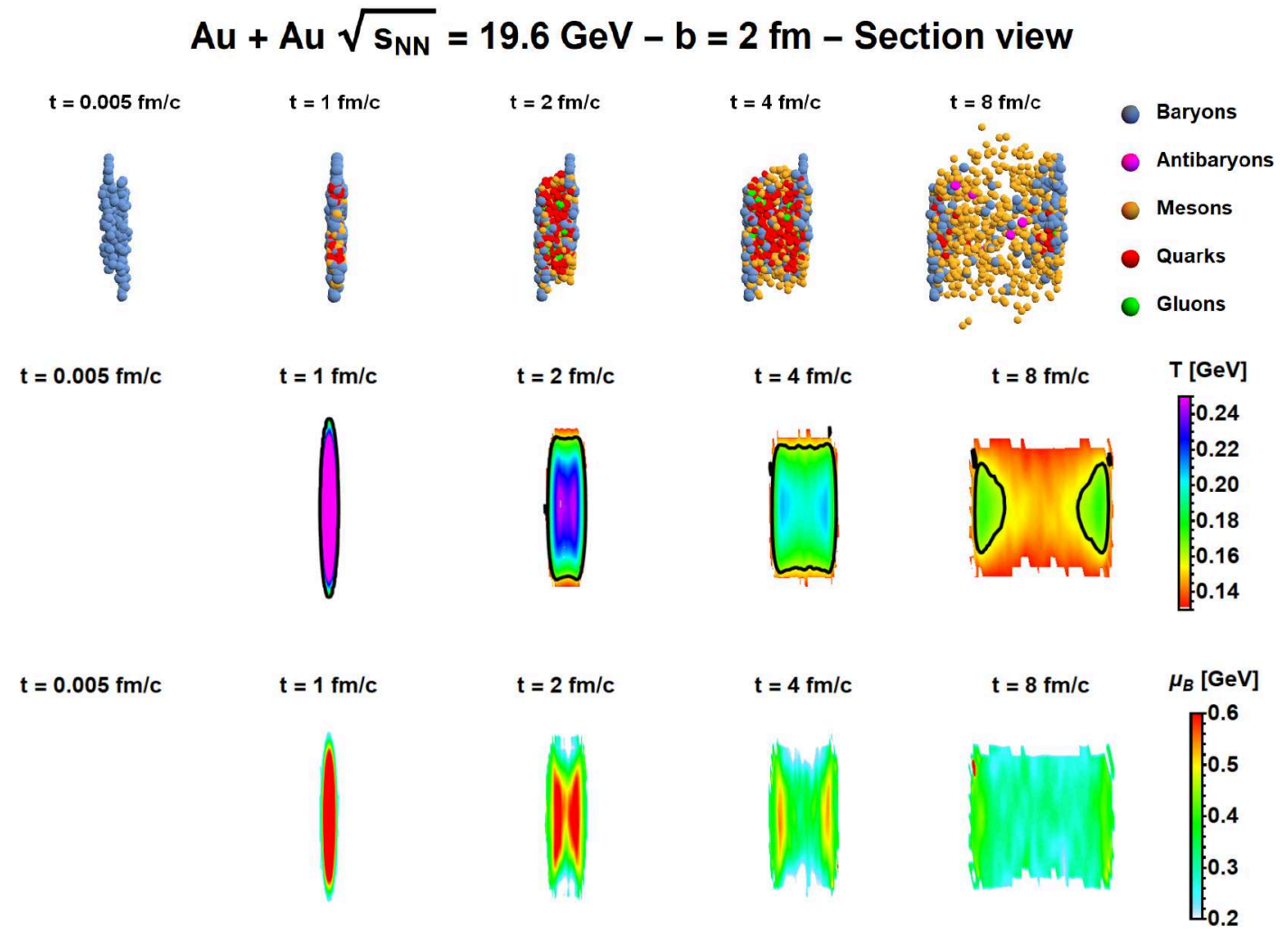

Figure 3.2: Illustration of the time evolution of central $A u+A u$ collisions (upper row, section view) at a collisional energy of $\sqrt{s_{N N}}=19.6 \mathrm{GeV}$ within the PHSD 5.0 [143]. The local temperature $T$ (middle row), baryon chemical potential $\mu_{B}$ (lower row), as extracted from the PHSD for $y \approx 0$. The black lines (middle row) indicate the critical temperature $T_{c}=0.158 \mathrm{GeV}$. The figure is taken from [143].

Here the left-hand sides consist of the energy density and baryon density from the lQCD Taylor expansion method, which depends on the unknown $T$ and $\mu_{B}$. The right-hand sides of these equations represent the energy density and baryon density evaluated in PHSD. In Eq. (112) the energy density from PHSD $\epsilon^{\mathrm{PHSD}}$ is divided by the function $r(x)$, which reads

$$
r(x)=\left\{\begin{array}{l}
\frac{x^{-1 / 3}}{2}\left[1+\frac{x \operatorname{arctanh} \sqrt{1-x}}{\sqrt{1-x}}\right] \text { for } x \leq 1 \\
\frac{x^{-1 / 3}}{2}\left[1+\frac{x \arctan \sqrt{x-1}}{\sqrt{x-1}}\right] \text { for } x \geq 1
\end{array} .\right.
$$

Here the anisotropy parameter $x$ is approximated as a function of the pressure components as $x=\left(P_{\|} / P_{\perp}\right)^{3 / 4} \cdot r(x)$ accounts for the anisotropy of the considered cell according to the shape generalized equation of state developed in Ref. [144]. The local temperature $T$ (middle row) and baryon chemical potential $\mu_{B}$ (lower row) extracted from the PHSD in the region with $y \approx 0$ are displayed in Fig. 3.2.

One can see from the time evolution of $\mathrm{Au}+\mathrm{Au}$ collisions - illustrated in the upper part of Fig. 3.2 - that the QGP is created in the early phase of the collisions and when the system expands, hadronization occurs. During the overlap phase the $T$ and $\mu_{B}$ are quite 


\section{$\mathrm{Pb}+\mathrm{Pb}$ 158AGeV - 5\% central}
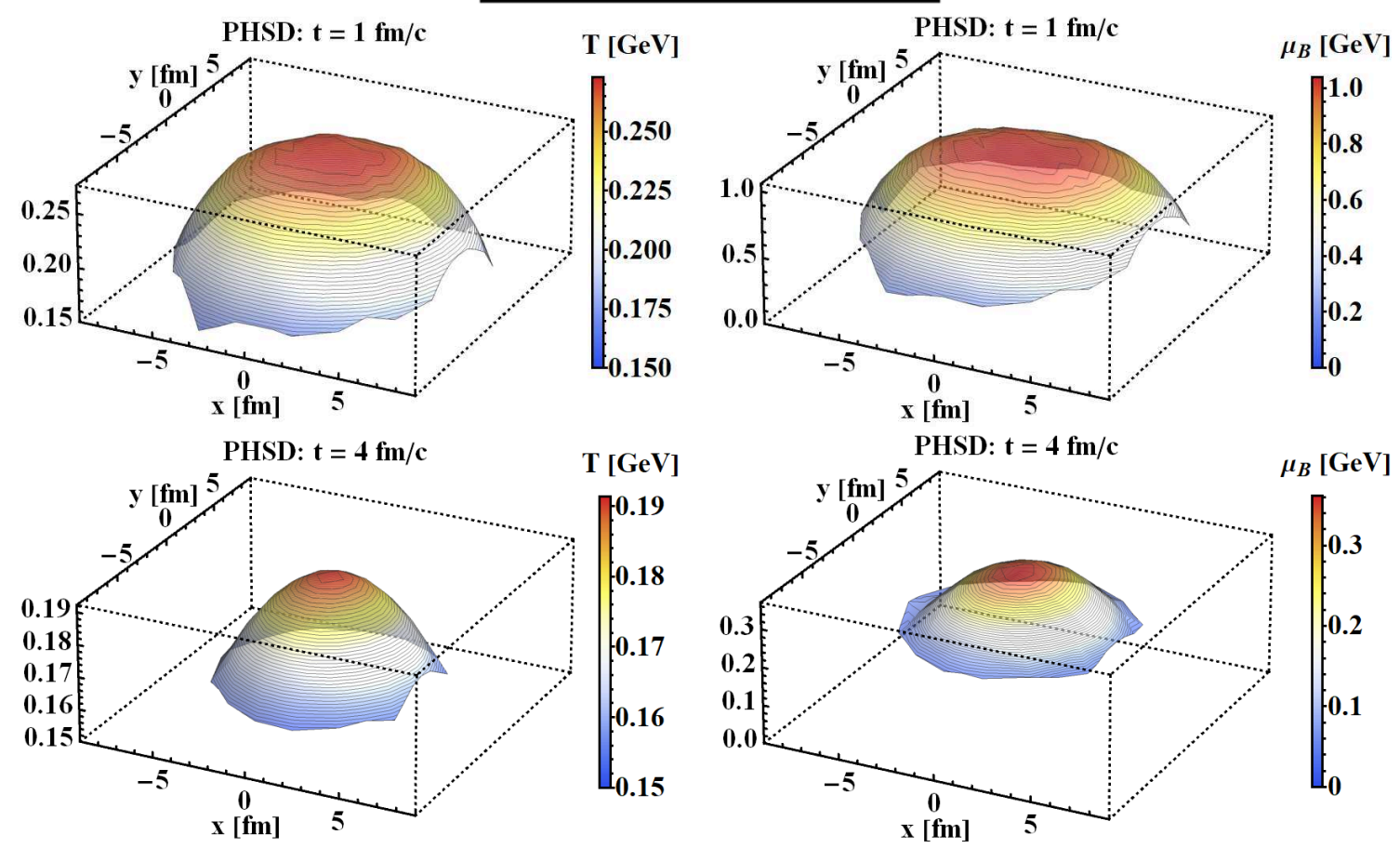

Figure 3.3: The $T$-profile (left) and $\mu_{B}$-profile (right) in $(x, y)$ at midrapidity $\left(\left|y_{\text {cell }}\right|<\right.$ 1) at 1 and $4 \mathrm{fm} / \mathrm{c}$ (from 0.5 to $6 \mathrm{fm} / \mathrm{c}$ for the $\mu_{B}$-profile) after the initial collision for $5 \%$ central $\mathrm{Pb}+\mathrm{Pb}$ collision at $158 \mathrm{~A} \mathrm{GeV}$ from PHSD5.0. The figure is taken from [1].

large ${ }^{3}$ compared to the averaged values and they decrease with time. Still even at $8 \mathrm{fm} / \mathrm{c}$ there are "hot spots" of QGP located at front surfaces of high rapidity.

Now let's look at the time evolution of the $T$ and $\mu_{B}$ distribution for cells having a temperature $T>T_{c}\left(\mu_{B}\right)$ at midrapidity $\left(\left|y_{\text {cell }}\right|<1\right)$ for $5 \%$ central $\mathrm{Pb}+\mathrm{Pb}$ collisions at 158 A GeV. Fig. 3.4 displays this distribution for times $t<2 \mathrm{fm} / \mathrm{c}, 2 \mathrm{fm} / \mathrm{c}<t<4 \mathrm{fm} / \mathrm{c}$ and $t>4 \mathrm{fm} / \mathrm{c}$. For early times $(t<2 \mathrm{fm} / \mathrm{c})$ the distribution peaks at a temperature of about $0.25 \mathrm{GeV}$ and a sizable chemical potential of about $0.6 \mathrm{GeV}$, while for times in the interval $2 \mathrm{fm} / \mathrm{c}<t<4 \mathrm{fm} / \mathrm{c}$ the maximum has dropped already to an average temperature $\sim$ $0.18 \mathrm{GeV}$ and a chemical potential of about $0.3 \mathrm{GeV}$. For later times $(t>4 \mathrm{fm} / \mathrm{c})$ the distribution (above $T_{c}$ ) essentially stays around $\mu_{B} \approx 0.25 \mathrm{GeV}$. As follows from Fig. 3.4 the $\mu_{B}$ probed around the transition temperature $T_{c}$ in the PHSD are in accordance with the expectation from statistical models, which for central $\mathrm{Pb}+\mathrm{Pb}$ collisions at $158 \mathrm{~A} \mathrm{GeV}$ predict $\mu_{B}=0.2489 \mathrm{GeV}$ [145].

By decreasing the collisional energy of the initial nuclei, one decreases the volume of the QGP produced in the heavy-ion collisions. The QGP energy fraction versus the total energy for $\mathrm{Au}+\mathrm{Au}$ at different collisional energies $\sqrt{s_{N N}}$ (accounting only the midrapidity region $|y|<0.5)$ is shown in Fig. 3.5. One can see that for high energies the QGP fraction is large compared to lower collisional energies where the volume of the QGP is small. While at high energy heavy-ion collisions the QGP phase appears suddenly after the initial pri-

$3 \quad$ We note that an extraction of the thermodynamic quantities for the strongly non-equilibrium initial stage is not a consistent procedure. 

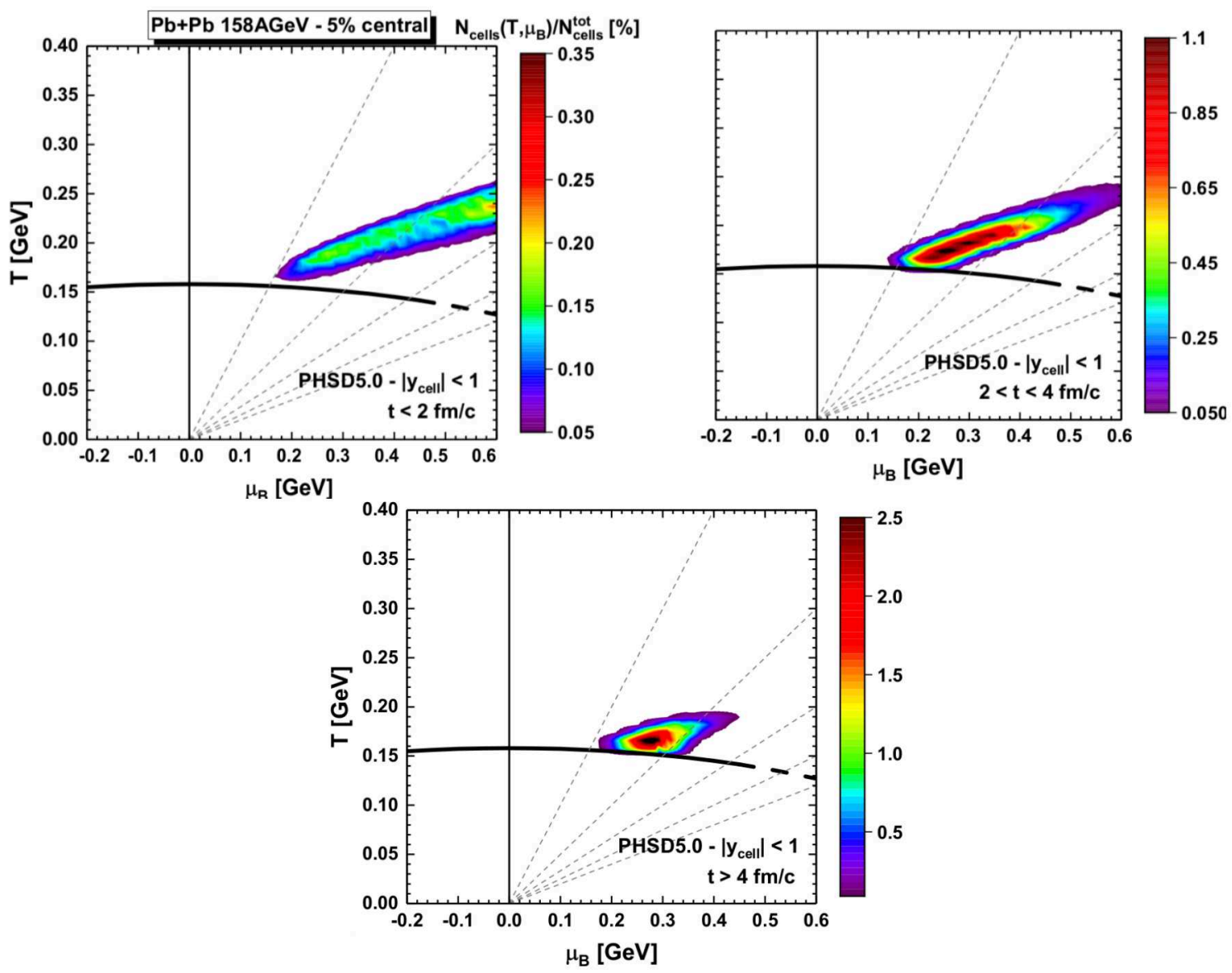

Figure 3.4: Distributions in $T$ and $\mu_{B}$ as extracted from the DQPM equation of state in a PHSD simulation of a central $\mathrm{Pb}+\mathrm{Pb}$ collision at $158 \mathrm{~A} \mathrm{GeV}$ for cells with a temperature $T>T_{c}\left(\mu_{B}\right)$ at midrapidity $\left(\left|y_{\text {cell }}\right|<1\right)$. The scale corresponds to the number of cells in the PHSD event in the considered bin in $T-\mu_{B}$ divided by the total number of cells in the corresponding time window (see legend). The solid black line is the DQPM phase boundary for orientation; the gray dashed lines indicate ratios of $\mu_{B} / T$ ranging from 1 to 5 while the vertical line corresponds to $\mu_{B}=0$. The figure is adopted from [1].

mary NN collision, at low energies it appearance is smoother since the passing time of the nuclei is longer. Consequently, at low energies the lifetime of the QGP is large, however, the QGP volume is tiny. Therefore the influence of the QGP on the dynamics is much lower compared to high-energy collisions where practically $90 \%$ of matter at midrapidity is in the QGP phase (at least for a short time).

\subsubsection{Bulk observables from relativistic nucleus-nucleus collisions}

Let us first examine within the PHSD approach the bulk observables, such as rapidity and transverse momentum distributions of different hadrons produced in central $\mathrm{Au}+\mathrm{Au}$ or $\mathrm{Pb}+\mathrm{Pb}$ collisions at lower and intermediates energies covered experimentally by the AGS (BNL) and the SPS (CERN). In this energy range the average baryon chemical potential $\mu_{B}$ is essentially finite - contrary to RHIC and LHC energies - and one might find some 


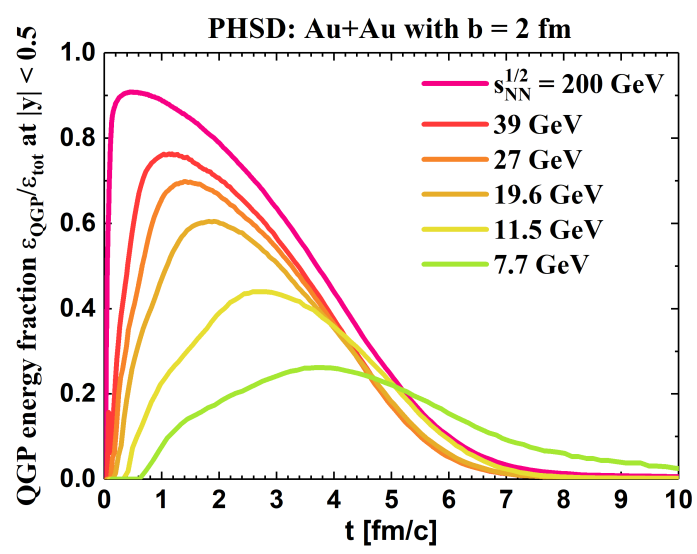

Figure 3.5: The QGP energy fraction from PHSD as a function of time $t$ in central (impact parameter $\mathrm{b}=2 \mathrm{fm}$ ) $\mathrm{Au}+\mathrm{Au}$ collisions for different collisional energies $\sqrt{s_{N N}}$ for $|y|<0.5$. The figure is taken from [7].

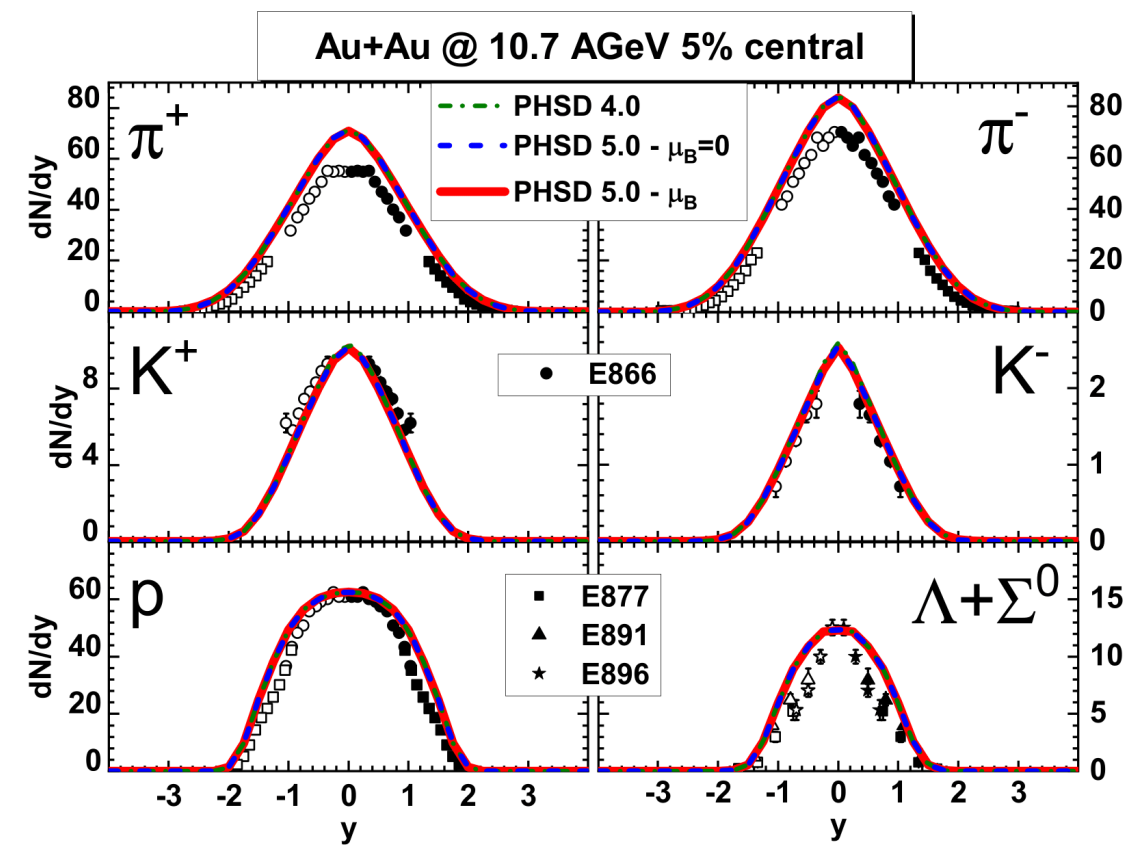

Figure 3.6: The rapidity distributions for $5 \%$ central $\mathrm{Au}+\mathrm{Au}$ collisions at $10.7 \mathrm{~A} \mathrm{GeV}$ for PHSD4.0 (green dot-dashed lines), PHSD5.0 with partonic cross sections and parton masses calculated for $\mu_{B}=0$ (blue dashed lines) and with cross sections and parton masses evaluated at the actual chemical potential $\mu_{B}$ in each individual space-time cell (red lines) in comparison to the experimental data from the E866 [146], E877 [147], E891 [148], E877 [149] and E896 [150] collaborations. All PHSD results are the same within the linewidth. The figure is taken from [1].

traces of the explicit $\mu_{B}$-dependence of the partonic cross sections in observables. Here we compare results for the rapidity distributions from the PHSD calculations based on the default DQPM parameters (PHSD4.0) [138] with the new PHSD5.0 including the differential partonic cross sections for the individual partonic channels at finite $\mathrm{T}$ and $\mu_{B}$ (cf. Ref. [1]). Actual results for hadronic rapidity distributions in case of $5 \%$ central $\mathrm{Au}+\mathrm{Au}$ collisions at $10.7 \mathrm{~A} \mathrm{GeV}$ are shown in Fig. 3.6 for PHSD4.0 (green dot-dashed lines), PHSD5.0 with partonic cross sections and parton masses calculated for $\mu_{B}=0$ (blue dashed lines), and 
with cross sections and parton masses evaluated at the actual chemical potential $\mu_{B}$ in each individual space-time cell (red lines) in comparison to the experimental data from the E866 [146], E877 [147], E891 [148], E877 [149] and E896 [150] collaborations. Here we consider the most abundant hadrons, i.e. pions, kaons, protons and neutral hyperons. The results for all the hadron species from the different versions of PHSD essentially coincide within linewidths as seen from Fig. 3.6. This approximate agreement implies that there is no sensitivity to the new partonic differential cross sections and parton masses employed. However, this effect can be explained by the low amount of QGP produced at this energy but the different PHSD calculations for $5 \%$ central $\mathrm{Pb}+\mathrm{Pb}$ collisions at $30 \mathrm{~A} \mathrm{GeV}$ in Fig. 3.7 for the hadronic rapidity distributions do not provide a different picture, too. Only

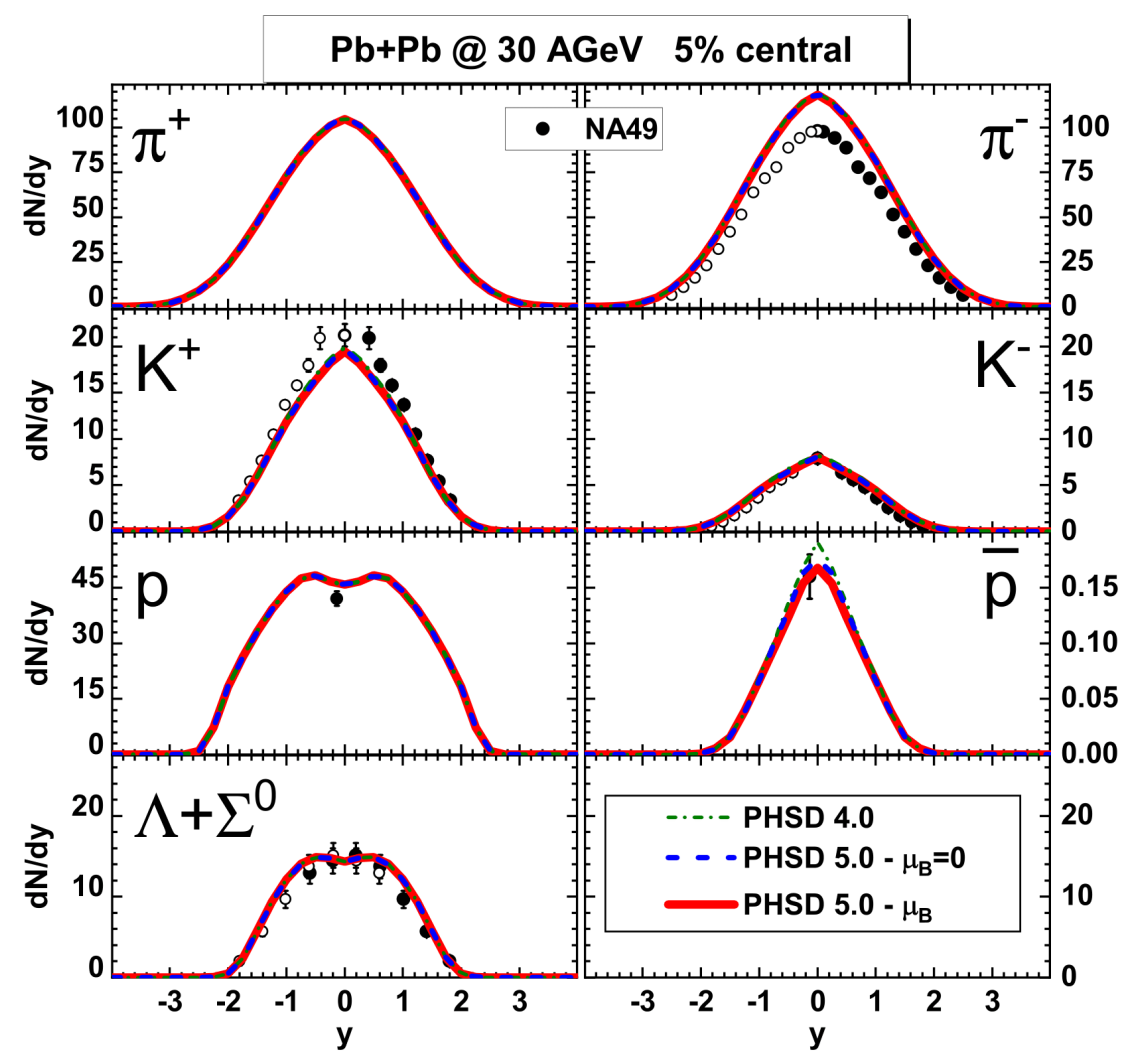

Figure 3.7: The rapidity distributions for $5 \%$ central $\mathrm{Pb}+\mathrm{Pb}$ collisions at $30 \mathrm{~A} \mathrm{GeV}$ for PHSD4.0 (green dot-dashed lines), PHSD5.0 with partonic cross sections and parton masses calculated for $\mu_{B}=0$ (blue dashed lines) and with cross sections and parton masses evaluated at the actual chemical potential $\mu_{B}$ in each individual space-time cell (solid red lines) in comparison to the experimental data from the NA49 Collaboration [151-153]. All PHSD results are practically the same within the linewidth. The figure is taken from [1].

when stepping up to the top SPS energy of $158 \mathrm{~A} \mathrm{GeV}$ one can identify a small difference in the antibaryon sector $\left(\bar{p}, \bar{\Lambda}+\bar{\Sigma}^{0}\right)$ in case of $5 \%$ central $\mathrm{Pb}+\mathrm{Pb}$ collisions (cf. Fig. 3.8).

So far at lower and intermediate energies we have not found any prominent sensitivity in hadronic rapidity distributions to differential parton cross sections, but the transverse dynamics needs to be investigated in addition. Hence, transverse momentum distributions for $5 \%$ central $\mathrm{Au}+\mathrm{Au}$ and $\mathrm{Pb}+\mathrm{Pb}$ collisions at $10.7 \mathrm{~A} \mathrm{GeV}, 30 \mathrm{~A} \mathrm{GeV}$ and 158 


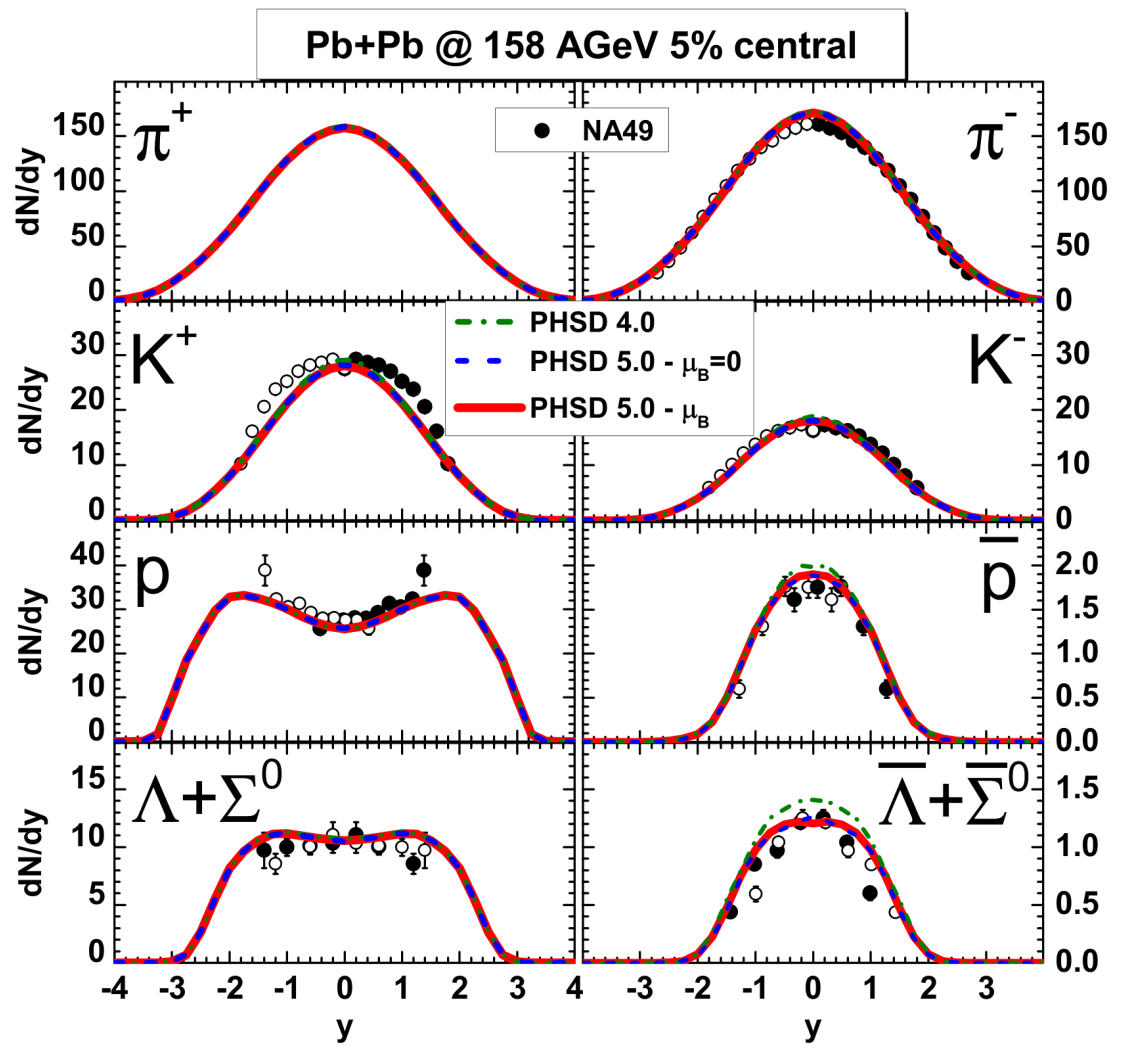

Figure 3.8: The rapidity distributions for $5 \%$ central $\mathrm{Pb}+\mathrm{Pb}$ collisions at $158 \mathrm{~A} \mathrm{GeV}$ for PHSD4.0 (green dot-dashed lines), PHSD5.0 with partonic cross sections and parton masses calculated for $\mu_{B}=0$ (blue dashed lines) and with cross sections and parton masses evaluated at the actual chemical potential $\mu_{B}$ in each individual space-time cell (red lines) in comparison to the experimental data from the NA49 Collaboration [154-157]. All PHSD results are the same within the linewidth except for the antibaryons. The figure is taken from [1].

A GeV, at an average rapidity $(|y|<0.5)$ are shown in Fig. 3.9 for PHSD4.0 (green lines), PHSD5.0 with parton cross sections and masses of partons calculated for $\mu_{B}=0$ (blue lines) and with cross sections and masses of partons estimated at the actual chemical potential $\mu_{B}$ in each separate cell of space-time (red lines) in comparison with experimental data from the collaborations E917 and E866 [158,159], and from the NA49 Collaboration [151-153, 155, 156, 160]. Here, the solid lines represent positively charged particles and dashed lines show results for negatively charged particles. We found that at $30 \mathrm{~A} \mathrm{GeV}$ there are practically no changes in the $p_{T}$ spectra for all versions of PHSD; it is only at $158 \mathrm{~A} \mathrm{GeV}$ that tiny changes in the $p_{T}$ spectra become noticeable for transverse momenta above about $2.5 \mathrm{GeV} /$ c. Moreover, for $10.7 \mathrm{~A} \mathrm{GeV} \mathrm{Au}+\mathrm{Au}$ collisions, we also find no visible changes in the $p_{T}$-spectra within the linewidth (cf. Fig. 3.9). These findings can be explained by the fact that at AGS and SPS energies the space-time volume of the partonic phase is too small even in central $\mathrm{Pb}+\mathrm{Pb}$ collisions such that one has practically no sensitivity to the microscopic collisional details in the partonic phase. However, this might change for ultra-relativistic collision systems where the QGP phase becomes dominant. We expect a dominantly partonic phase in central $\mathrm{Au}+\mathrm{Au}$ collisions at $\sqrt{s_{N N}}=200 \mathrm{GeV}$ especially when gating on midrapidity. However, the differences between PHSD4.0 and 

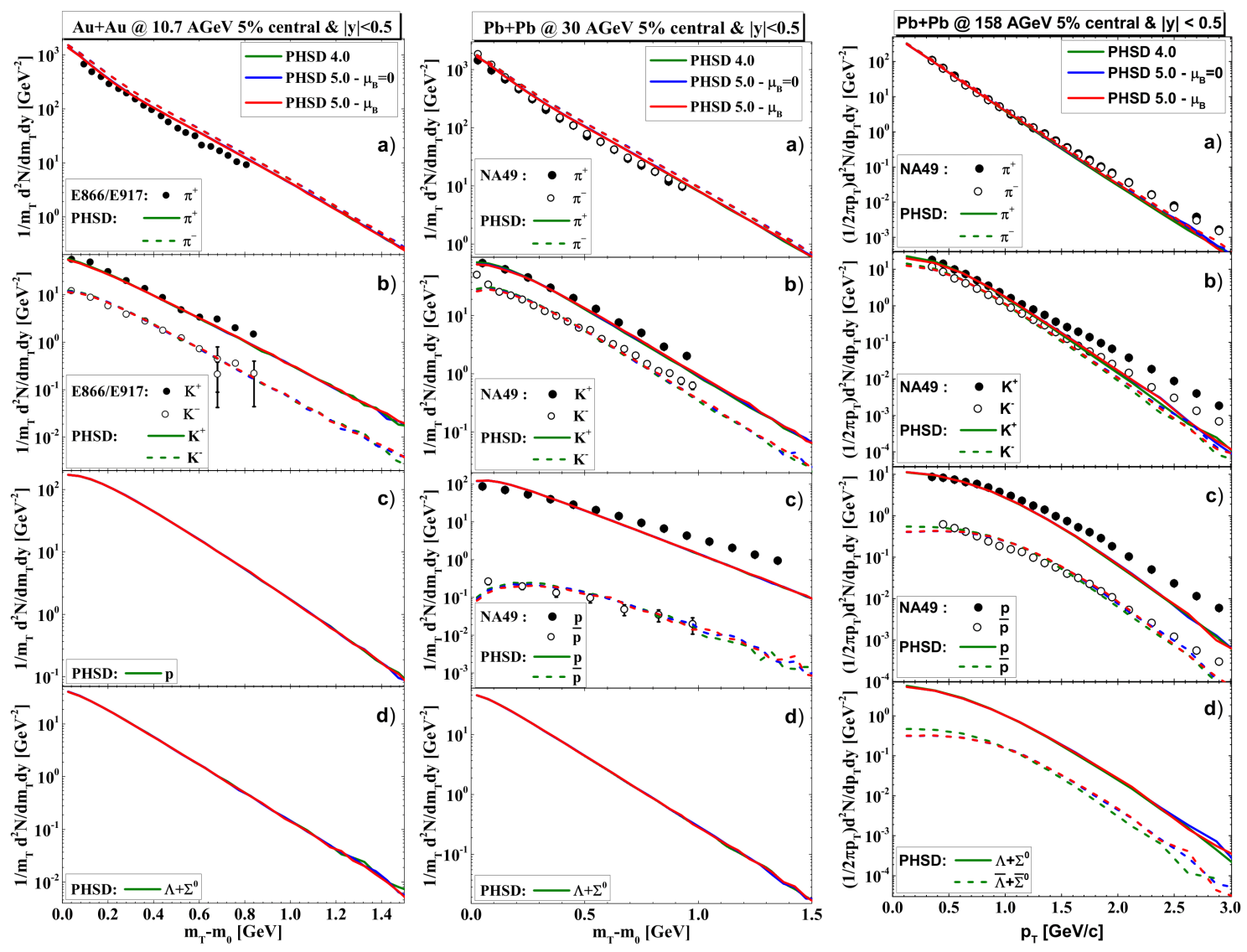

Figure 3.9: The transverse momentum distributions for $5 \%$ central $\mathrm{Au}+\mathrm{Au}$ collisions at $11 \mathrm{~A} \mathrm{GeV}$ (left panel) and $\mathrm{Pb}+\mathrm{Pb}$ collisions at $30 \mathrm{~A} \mathrm{GeV}$ (middle panel) and $158 \mathrm{~A}$ $\mathrm{GeV}$ (right panel), in midrapidity $(|y|<0.5)$ for PHSD4.0 (green lines), PHSD5.0 with partonic cross sections and parton masses calculated for $\mu_{B}=0$ (blue lines) and with cross sections and parton masses evaluated at the actual chemical potential $\mu_{B}$ in each individual space-time cell (red lines) in comparison to the experimental data from the E917 and E866 collaborations [158, 159] (left), from the NA49 Collaboration [151-153] (middle) and $[155,156,160]$ (right). The figure is taken from [1].

PHSD5.0 (with and without $\mu_{B}$-dependence) in the hadronic rapidity distributions for $5 \%$ central $\mathrm{Au}+\mathrm{Au}$ collisions turn out to be rather small for mesons $\left(\pi^{ \pm}, K^{ \pm}\right)$and also for baryons and antibaryons $\left(p, \bar{p}, \Lambda+\Sigma^{0}, \bar{\Lambda}+\bar{\Sigma}^{0}\right)$ (cf. Fig. 3.11 (left)) such that no robust conclusion on the partonic collisional dynamics can be drawn even in this case.

This also holds true for the transverse momentum distributions at midrapidity $(|y|<0.5)$ for these collisions when comparing the results from the different PHSD versions with each other and the data from the PHENIX [162] and STAR [163] collaborations in Fig. 3.11 (right). Only for high transverse momenta small differences can be seen with the tendency to improve the description of the data in the novel versions of PHSD5.0 with the microscopic differential partonic cross sections. 


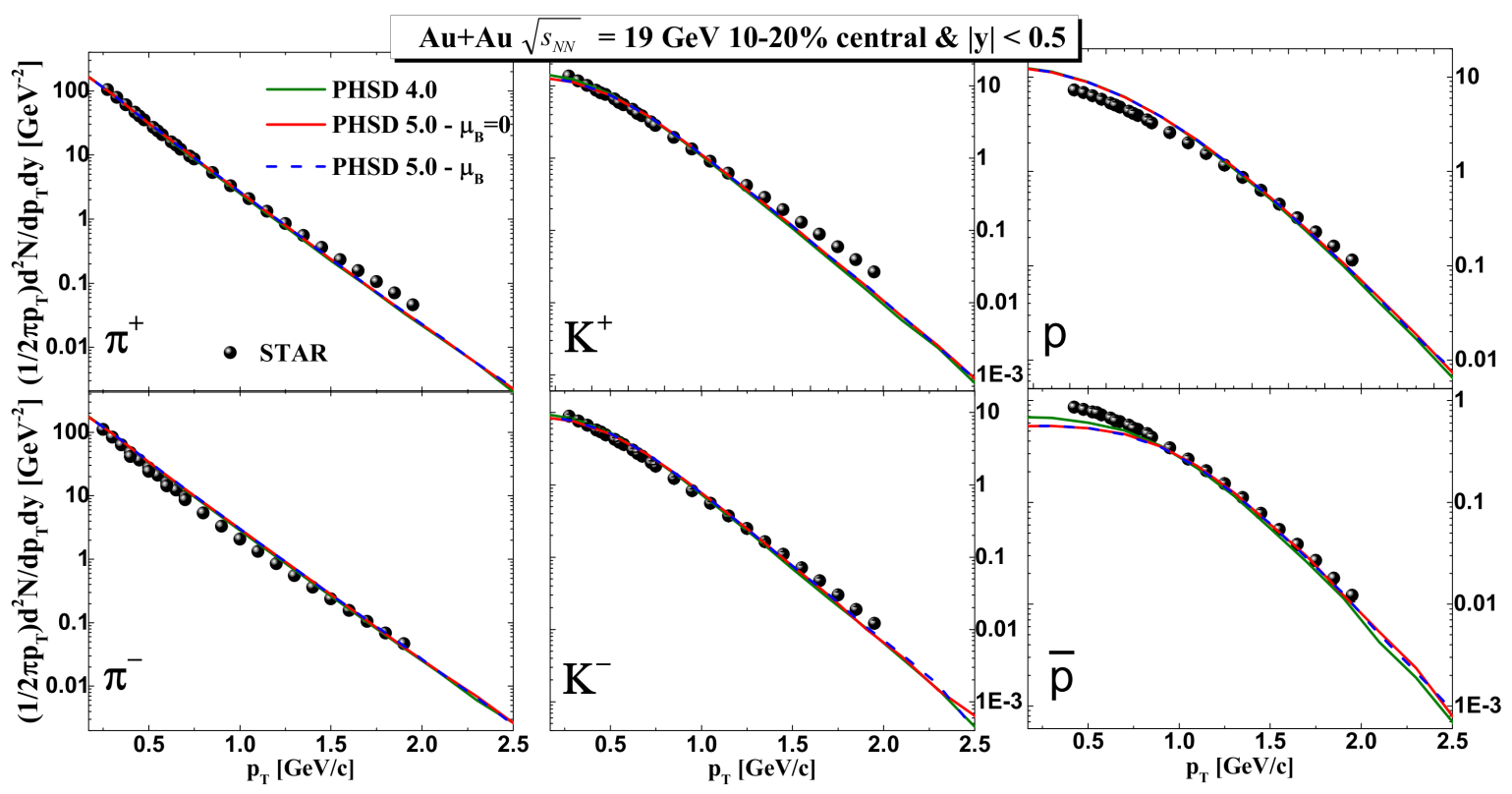

Figure 3.10: Transverse momentum distributions for 10-20\% central $\mathrm{Au}+\mathrm{Au}$ collisions at $\sqrt{s_{N N}}=19 \mathrm{GeV}$ and midrapidity $(|\mathrm{y}|<0.5)$ for PHSD4.0 (green lines), PHSD5.0 with partonic cross sections and parton masses calculated for $\mu_{B}=0$ (blue dashed lines) and with cross sections and parton masses evaluated at the actual chemical potential $\mu_{B}$ in each individual space-time cell (red lines) in comparison to the experimental data from the STAR collaboration [161].

\subsubsection{Asimuthal anisotropy in the HICs}

The azimuthal anisotropy of the particle distribution is one of the important observables characterizing the collective effects at the initial stage of quark-gluon matter formation and expansion during the collision of relativistic nuclei. In non-central nuclear collisions the direction of the beam axis and the impact parameter determine the reaction plane. The observed particle multiplicity as a function of the azimuthal angle with respect to the reaction plane in each event provides information on the collision dynamics at an early stage $[166,167]$. The initial overlap region of the nuclei at non-zero impact parameter has an elliptical shape. In the process of thermalization of the created matter, due to the spatial anisotropy of the system, pressure gradients are created with a maximum along the smaller axis of the ellipse and a minimum along the larger axis. As a result, the azimuthal momentum distribution of the particles becomes anisotropic (See Fig. 3.12). Quantitatively, the elliptic flow is characterized by the second coefficient $v_{2}$ in the Fourier series expansion of particle distribution along the azimuthal angle relative to the reaction plane.

Due to the reflection symmetry in the Fourier decomposition of the azimuthal angular distribution in a Fourier series with a sufficiently large number of number of particles in the event, with only cosine terms:

$$
E \frac{d^{3} N}{d^{3} p}=\frac{d^{3} N}{d y p_{T} d p_{T} d \phi}=\frac{1}{2 \pi} \frac{d^{2} N}{p_{T} d p_{T} d y}\left(1+2 \sum_{n=1}^{\infty} v_{n}\left(y, p_{T}\right) \cos \left[n\left(\phi-\Psi_{R P}\right)\right]\right)
$$



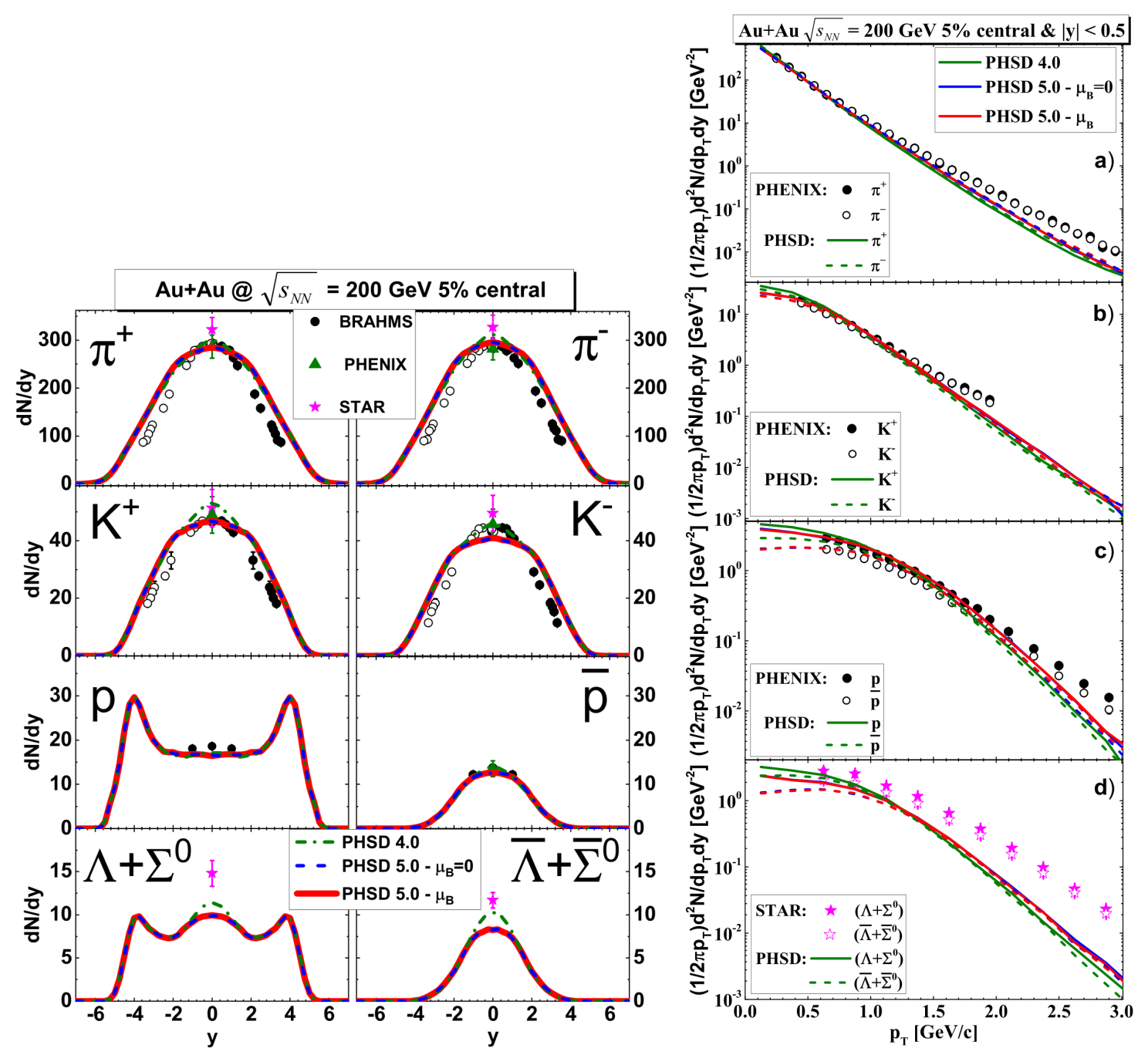

Figure 3.11: The rapidity distributions (left) and transverse momentum distributions (right) for $5 \%$ central $\mathrm{Au}+\mathrm{Au}$ collisions at $\sqrt{s_{N N}}=200 \mathrm{GeV}$ for PHSD4.0 (green dotdashed lines), PHSD5.0 with partonic cross sections and parton masses calculated for $\mu_{B}=0$ (blue dashed lines) and with cross sections and parton masses evaluated at the actual chemical potential $\mu_{B}$ in each individual space-time cell (red lines) in comparison to the experimental data from the BRAHMS [164,165], PHENIX [162] and STAR [163] collaborations. The figure is taken from [1].

where $E$ is the energy of particle, $p$ the total momentum, $p_{T}$ the transverse momentum, $y$ the rapidity, $\phi$ the azimuthal angle and $\Psi_{R P}$ the reaction plane angle. We are interested in the Fourier coefficients of this expansion:

$$
v_{n}=<\cos \left[n\left(\phi-\Psi_{R P}\right)\right]>
$$

where averaging over the particles, summation over all events for every $\left(p_{T}, \mathrm{y}\right)$ bin is performed. Further on we consider the first two coefficients $v_{1}$ and $v_{2}$, which are known as directed and elliptic flow, respectively. These first two flow coefficients carry information on the early stages of the collision. The directed flow manifests itself in the flattening of spectra, predominantly in $\mathrm{A}+\mathrm{A}$ versus $\mathrm{p}+\mathrm{p}$ collisions, at small transverse kinetic energies. There are different methods for measuring the azimuthal anisotropy coefficient, which have 
their own advantages and disadvantages. The first method uses the determination of the angle of the reaction plane in each event [168]. In this case, the sign of the coefficient $v_{2}$ is known, if the angle of the reaction plane is determined by the first harmonic. The second and third methods are the cumulant method and the Lee-Young zero method [168]. In this case, the sign of $v_{2}$ cannot be determined. Here we consider first method also known as the standard event plane method [168] described in Appendix C.
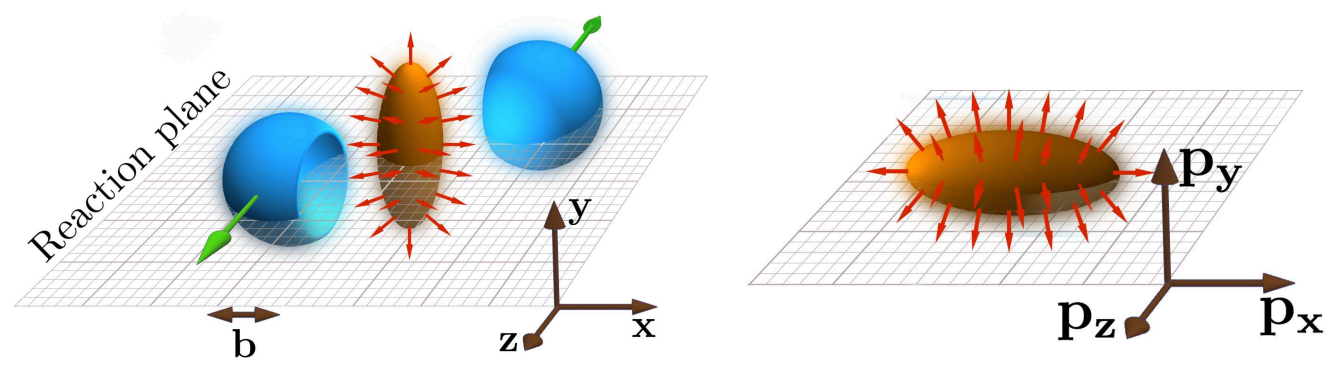

Figure 3.12: (Left) A non-central collision of two nuclei with impact factor $b$ leads to an almond-shaped interaction volume. (Right) The initial spatial anisotropy with respect to the reaction plane evolves under pressure gradients into a momentum anisotropy of the produced particles.

\subsubsection{Directed Flow $v_{1}$}

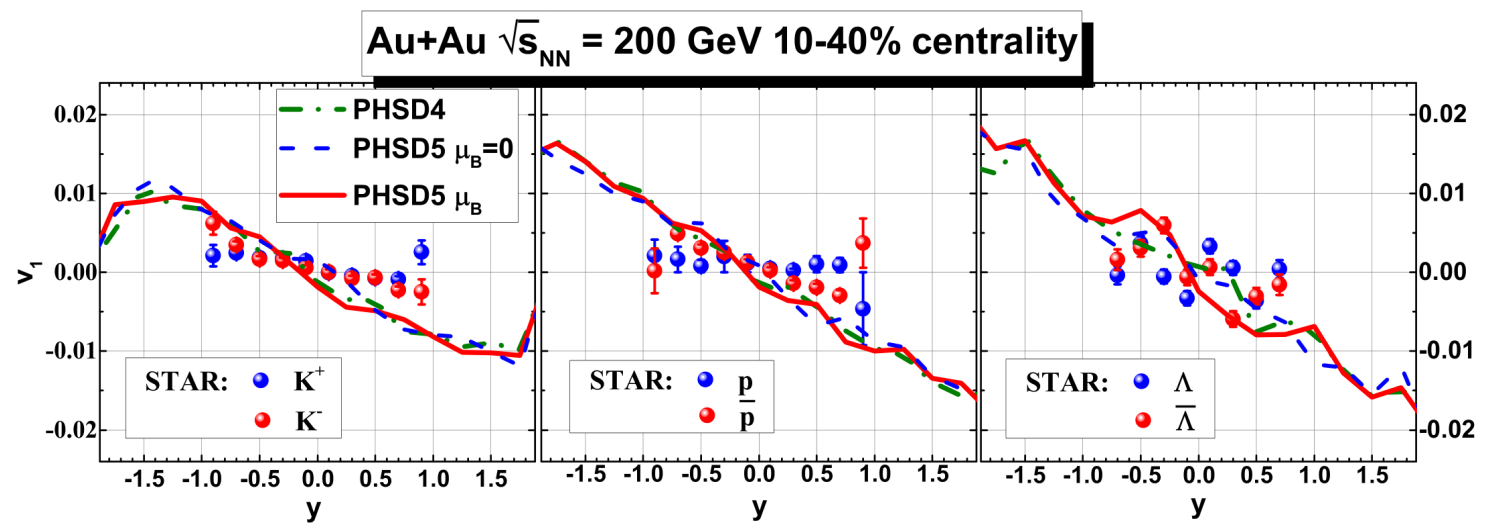

Figure 3.13: Directed flow of identified hadrons as a function of rapidity at $\sqrt{s_{N N}}=200$ $\mathrm{GeV}$ for PHSD4.0 (green lines), PHSD5.0 with partonic cross sections and parton masses calculated for $\mu_{B}=0$ (blue dashed lines) and with cross sections and parton masses evaluated at the actual chemical potential $\mu_{B}$ in each individual space-time cell (solid red lines) in comparison to the experimental data of the STAR Collaboration [169].

Now, we test the traces of $\mu_{B}$-dependencies of the QGP interaction cross sections in collective observables such as the directed flow $v_{1}$ considering again three cases of the PHSD as discussed above.

Figure 3.14 depicts the directed flow $v_{1}$ of identified hadrons $\left(K^{ \pm}, p, \bar{p}, \Lambda+\Sigma^{0}, \bar{\Lambda}+\bar{\Sigma}^{0}\right)$ versus rapidity for $\sqrt{s_{N N}}=27 \mathrm{GeV}$. One can see a good agreement between PHSD results and experimental data from the STAR collaboration [169]. However, the different versions of PHSD for the $v_{1}$ coefficient show a quite similar behavior; only antihyperons show a 


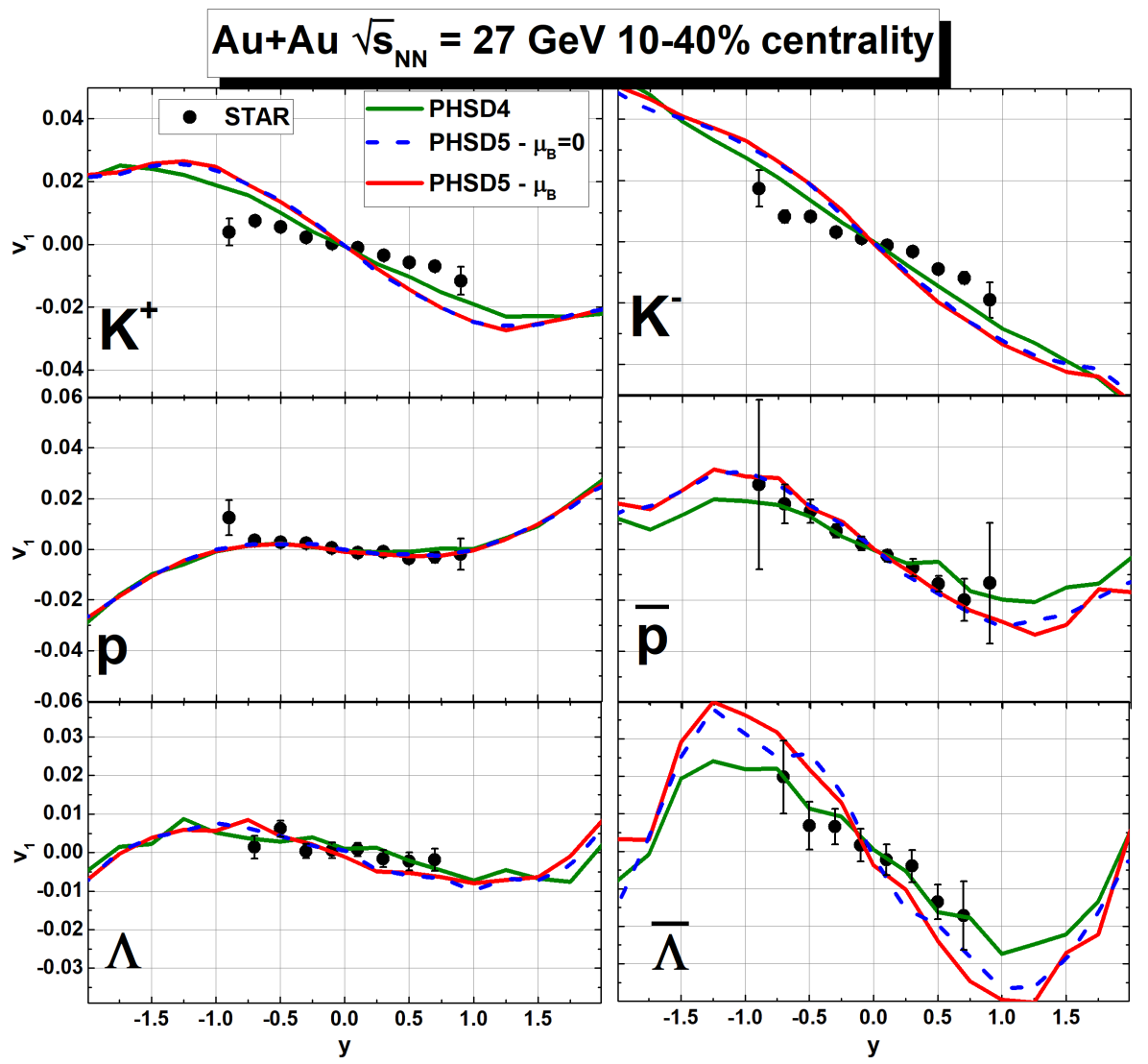

Figure 3.14: Directed flow of identified hadrons as a function of rapidity at $\sqrt{s_{N N}}=27$ GeV for PHSD4.0 (green lines), PHSD5.0 with partonic cross sections and parton masses calculated for $\mu_{B}=0$ (blue dashed lines) and with cross sections and parton masses evaluated at the actual chemical potential $\mu_{B}$ in each individual space-time cell (red lines) in comparison to the experimental data of the STAR Collaboration [169].

slightly different flow. This supports again the finding that strangeness, and in particular anti-strange hyperons, are the most sensitive probes for the QGP properties. To check further effects in the partonic phase on the directed flow we consider higher collision energy. The directed flow $v_{1}$ of identified hadrons $\left(K^{ \pm}, p, \bar{p}, \Lambda+\Sigma^{0}, \bar{\Lambda}+\bar{\Sigma}^{0}\right)$ versus rapidity at $\sqrt{s_{N N}}$ $=200 \mathrm{GeV}$ is demonstrated in Figure 3.13. The difference between the PHSD lines is less pronounced than for $\sqrt{s_{N N}}=27 \mathrm{GeV}$. Note that the difference between positive and negative charged hadrons $\left(h^{+}\right.$and $\left.h^{-}\right)$is small for $\sqrt{s_{N N}}=200 \mathrm{GeV}$, thus we considered the averaged $v_{2}$ of charged particles: $v_{2}(h)=\frac{v_{2}\left(h^{+}\right)+v_{2}\left(h^{-}\right)}{2}$.

\subsubsection{Elliptic Flow $v_{2}$}

The influence of transport coefficients (mainly shear $\eta$ and bulk $\zeta$ viscosities) to the QGP evolution in HICs has been widely studied in the hydrodynamic simulations $[118,170,171]$ and in the Bayesian analysis [89], where an explicit dependence of the elliptic flow $v_{2}$ on the transport properties of the QGP has been manifested. In this section, we consider the results for the elliptic flow of charged hadrons produced in symmetric $\mathrm{A}+\mathrm{A}$ collisions within the PHSD5.0 with and without $\mu_{B}$-dependence and compare to the results from PHSD4.0. To track the effect of the QGP phase on the final observables we consider a 
channel decomposition: we group the final hadrons according to their origin by considering three channels: i) hadrons coming from the QGP without rescattering in the hadronic phase, ii) hadrons coming from strings, and iii) hadrons coming from mesonic and baryonic resonance decays. This allows to compare the flow coefficients between the considered channels of final particles and see an effect of the QGP dynamics for final particles in a specific channel.

The left plots '(a)' in Figures 3.15 and 3.16 display the actual results for the charged hadron elliptic flow as a function of pseudo-rapidity $\eta$ (Figure 3.15) and of transverse momentum $p_{T}$ (Figure 3.16) for minimum bias $\mathrm{Au}+\mathrm{Au}$ collisions at $\sqrt{s_{N N}}=200 \mathrm{GeV}$ for PHSD4.0 (green lines), PHSD5.0 with partonic cross sections and parton masses calculated for $\mu_{B}=0$ (blue dashed lines), and with cross sections and parton masses evaluated at the actual chemical potential $\mu_{B}$ in each individual space-time cell (red lines) in comparison to the experimental data from the STAR collaboration [172] (solid stars) and PHOBOS [173] (solid dots). The difference between results for PHSD4.0 and PHSD5.0 is more pronounced for $v_{2}$ versus $p_{T}$ in case of charged hadrons for high $p_{T}>0.5 \mathrm{GeV}$, than for $v_{2}\left(p_{T}\right)$ versus $\eta$.
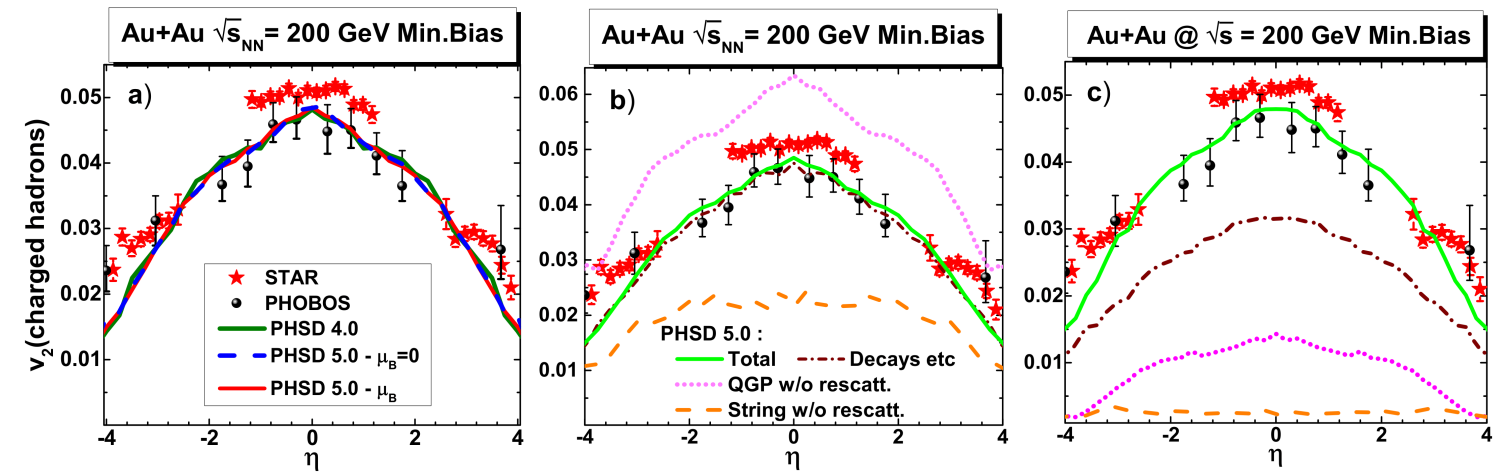

Figure 3.15: Left (a): elliptic flow of charged hadrons as a function of pseudo-rapidity $\eta$ for minimum bias $\mathrm{Au}+\mathrm{Au}$ collisions at $\sqrt{s_{N N}}=200 \mathrm{GeV}$ for PHSD4.0 (solid green line), PHSD5.0 with partonic cross sections and parton masses calculated for $\mu_{B}=0$ (blue dashed line), and with the actual $\mu_{B}$ (solid red line) in comparison to the experimental data from STAR [172] (red stars) and PHOBOS [173] (spheres) Collaborations. Middle (b): individual contributions to $v_{2}$ without their relative weights to the total $v_{2}$, which are indicated by a green solid line for PHSD5.0 with $\mu_{B}$ : the magenta dotted line corresponds to the final hadrons coming from the QGP without rescattering in the hadronic phase, the orange dashed line indicates the $v_{2}$ of hadrons coming from strings while the brown dotdashed line shows the $v_{2}$ of hadrons coming from mesonic and baryonic resonance decays. Right (c): individual contributions to $v_{2}$ including their relative weights to the total $v_{2}$.

The channel composition of $v_{2}$ for PHSD5.0 - with cross sections and parton masses evaluated at the actual chemical potential $\mu_{B}$ in each individual space-time cell - is shown in the middle plots '(b)' of Figures 3.15 and 3.16. We sorted the particles according to their production channels into three parts as discussed above: the magenta dotted line corresponds to the final hadrons coming from the QGP without rescattering in the hadronic phase, the orange dashed line indicates the $v_{2}$ of hadrons coming from strings (without further rescattering) while the brown dot-dashed line shows the $v_{2}$ of hadrons coming 


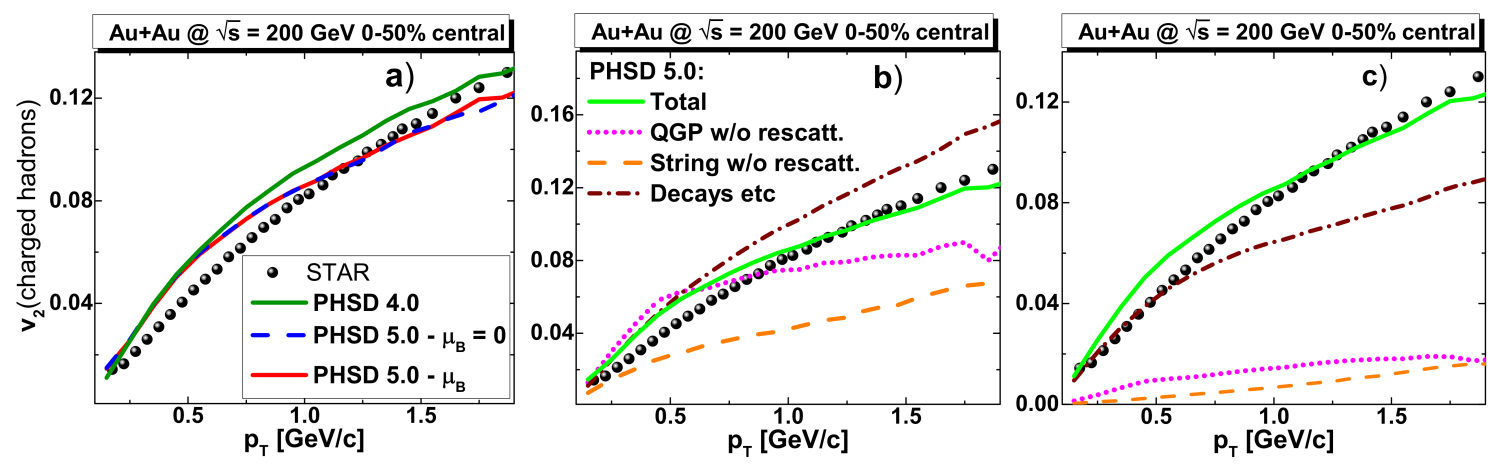

Figure 3.16: Elliptic flow of charged hadrons as a function of $p_{T}$ for $0-50 \%$ central $\mathrm{Au}+\mathrm{Au}$ collisions at $\sqrt{s_{N N}}=200 \mathrm{GeV}$ in comparison to the experimental data from the STAR Collaboration [172] (spheres). The line description is similar to Fig. 3.15.

from mesonic and baryonic resonance decays. One can see a large difference between the averaged elliptic flow for the different channels: the $v_{2}$ of hadrons from string decay is the lowest since string production occurs dominantly at the initial phase of the heavy-ion collision; the $v_{2}$ of hadrons from the QGP is the largest versus $\eta$ as follows from the middle Part '(b)' of Figure 3.15. However, this is mainly due to the low $p_{T}$ hadrons which give a larger contribution to $v_{2}(\eta)-$ cf. the middle part '(b)' of Figure 3.16. Here, the high $p_{T}$ hadrons from the QGP show a lower $v_{2}$ than those coming from strings or resonance decays.

The right parts '(c)' of Figures 3.15 and 3.16 present the individual contributions to $v_{2}$ including their relative weights to the total $v_{2}$. It shows that the properly weighted channel decomposition of $v_{2}$ looks rather different - the contribution of the hadrons from the QGP is now small since most of them rescatter in the hadronic phase, i.e., the relative fraction of hadrons directly coming from QGP hadronization is very small. The total $v_{2}$ is dominated by the hadrons coming from the decay of resonances. The fraction of hadrons from string decays is very small due to the fact that strings are formed mainly in the beginning of collisions, and a very small fraction of hadrons can survive directly. Thus, the information in $v_{2}$ about the QGP properties is washed out to a large extent by final hadronic interactions.

Figure 3.17 demonstrates the elliptic flow of identified hadrons $\left(K^{ \pm}, p, \bar{p}, \Lambda+\Sigma^{0}, \bar{\Lambda}+\bar{\Sigma}^{0}\right)$ as a function of $p_{T}$ at $\sqrt{s_{N N}}=27 \mathrm{GeV}$ for PHSD4.0 (green lines), PHSD5.0 with partonic cross sections and parton masses calculated for $\mu_{B}=0$ (blue dashed lines) and with cross sections and parton masses evaluated at the actual chemical potential $\mu_{B}$ in each individual space-time cell (red lines) in comparison to the experimental data of the STAR Collaboration [174]. Similar to the directed flow shown in Figure 3.14, the elliptic flow from all three cases for PHSD shows a rather similar behavior, the differences are very small (within the statistics achieved here). Only antiprotons and antihyperons show a small decrease of $v_{2}$ at larger $p_{T}$ for PHSD5.0 compared to PHSD4.0, which can be attributed to the explicit $\sqrt{s}$-dependence and different angular distribution of partonic cross sections in the PHSD5.0. We note that the underestimation of $v_{2}$ for protons and $\Lambda$ 's we attribute to the details of the hadronic vector potential involved in this calculations which seems to underestimate repulsion. 


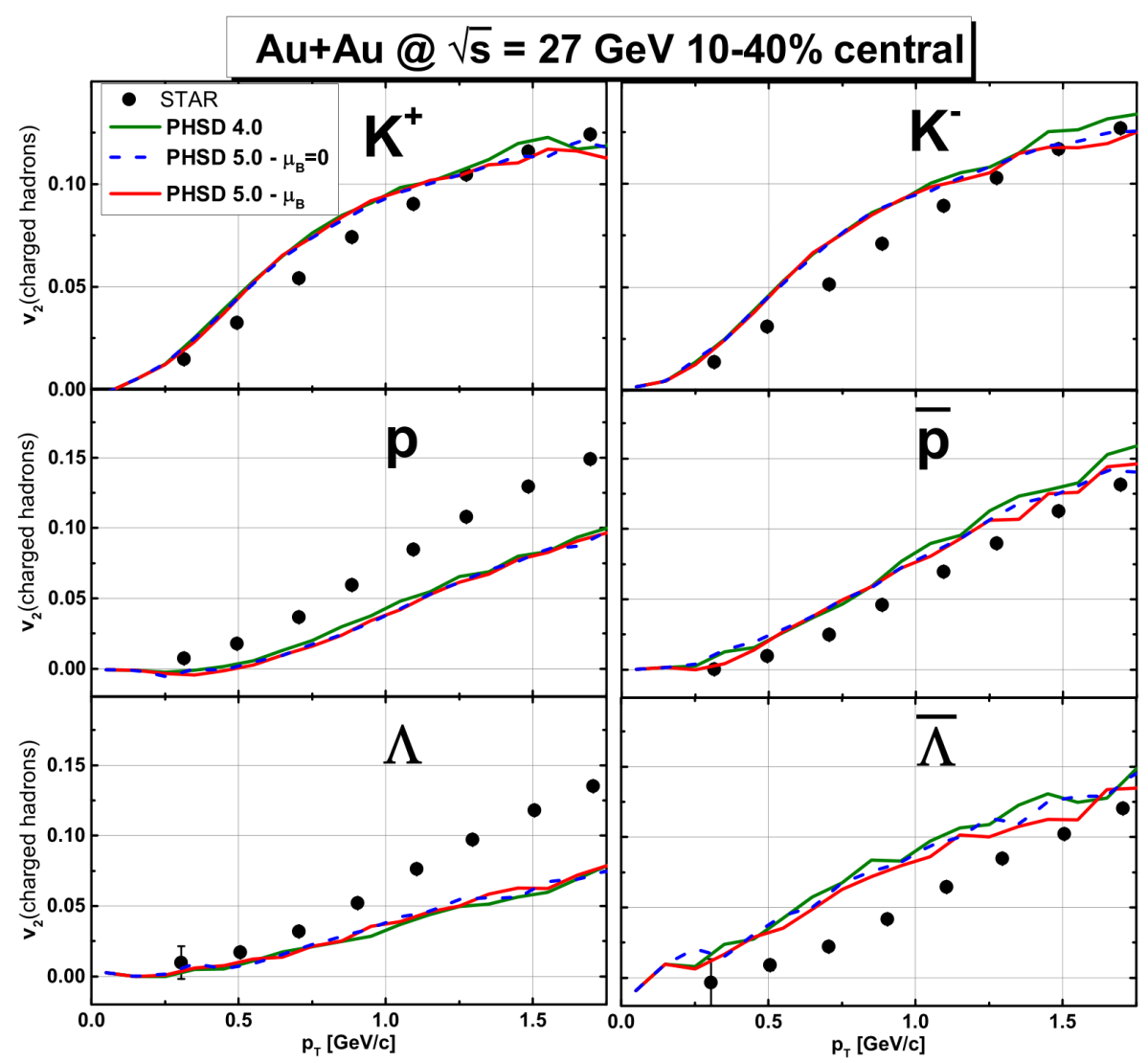

Figure 3.17: Elliptic flow of identified hadrons $\left(K^{ \pm}, p, \bar{p}, \Lambda+\Sigma^{0}, \bar{\Lambda}+\bar{\Sigma}^{0}\right)$ as a function of $p_{T}$ at $\sqrt{s_{N N}}=27 \mathrm{GeV}$ for PHSD4.0 (green lines), PHSD5.0 with partonic cross sections and parton masses calculated for $\mu_{B}=0$ (blue dashed lines) and with cross sections and parton masses evaluated at the actual chemical potential $\mu_{B}$ in each individual space-time cell (red lines) in comparison to the experimental data of the STAR Collaboration [174]. 


\subsection{Conclusions to Section 3}

In this chapter we have studied the influence of the baryon chemical potential $\mu_{B}$ on the properties of the QGP in equilibrium as well as the QGP created in heavy-ion collisions also far from equilibrium. For the description of the QGP we employed the extended effective DQPM that is matched to reproduce the lQCD crossover equation-of-state versus temperature $T$ and at finite baryon chemical potential $\mu_{B}$.

We based our study of the non-equilibrium QGP - as created in heavy-ion collisions on the extended PHSD transport approach in which i) the masses and widths of quarks and gluons depend on $T$ and $\mu_{B}$ explicitly; ii) the partonic interaction cross sections are obtained by calculations of the leading order Feynman diagrams from the DQPM and explicitly depend on the invariant energy $\sqrt{s}$, temperature $T$ and baryon chemical potential $\mu_{B}$. This extension is realized in the full version of PHSD5.0 [1].

In order to investigate the traces of the $\mu_{B}$-dependence of the QGP in observables, the results of PHSD5.0 with $\mu_{B}$-dependence have been compared to the results of PHSD5.0 for $\mu_{B}=0$ as well as with PHSD4.0 where the masses/width of quarks and gluons as well as their interaction cross sections depend only on $T$ following Ref. [84].

We have presented the PHSD results for different observables: (i) rapidity and $p_{T}$ distributions of identified hadrons for symmetric $\mathrm{Au}+\mathrm{Au}$ and $\mathrm{Pb}+\mathrm{Pb}$ collisions at energies of $30 \mathrm{AGeV}$ (future NICA energy) as well as for the top RHIC energy of $\sqrt{s_{N N}}=200 \mathrm{GeV}$; (ii) directed flow $v_{1}$ of identified hadrons for $A u+A u$ at invariant energy $\sqrt{s_{N N}}=27 \mathrm{GeV}$ and $200 \mathrm{GeV}$; (iii) elliptic flow $v_{2}$ of identified hadrons for $A u+A u$ at invariant energies $\sqrt{s_{N N}}=27$ and $200 \mathrm{GeV}$.

The comparison of the 'bulk' observables for A+A collisions within the three setting of PHSD has illuminated that they manifest a quite low sensitivity to the $\mu_{B^{-}}$dependences of parton properties (masses and widths) and their interaction cross sections such that the results from PHSD5.0 with and without $\mu_{B}$ were very close to each other. Only in the case of kaons, antiprotons $\bar{p}$ and antihyperons $\bar{\Lambda}+\bar{\Sigma}^{0}$, a small difference between PHSD4.0 and PHSD5.0 could be seen at top SPS and top RHIC energies.

A similar trend has been found for very asymmetric collisions of $\mathrm{C}+\mathrm{Au}$ : a small sensitivity to the partonic scatterings was found in the kaon and antibaryon rapidity distributions, too [1]. We find only small differences between PHSD4.0 and PHSD5.0 results on the hadronic observables considered here at high as well as at intermediate energies. This is related to the fact that at high energies, where the matter is dominated by the QGP, one probes a very small baryon chemical potential $\mu_{B}$ in central collisions at midrapidity, while, with decreasing energy, where $\mu_{B}$ becomes larger, the fraction of the QGP drops rapidly, such that in total the final observables are dominated by the hadrons which participated in hadronic rescattering and thus the information about their QGP origin is washed out or lost.

We have demonstrated that the $\mu_{B}$-dependence of QGP interactions is more pronounced in observables for strange hadrons, kaons and especially anti-strange hyperons, as well as for antiprotons. This gives an experimental hint for the search of $\mu_{B}$ traces of the QGP for experiments at the future NICA accelerator, even if it will be a very challenging 
3. Dynamical evolution of QCD matter from transport simulations

experimental task. 


\section{Critical end point and 1st order phase transition of QCD matter within the PNJL model}

This Chapter is devoted to the evaluation of transport coefficients of the QGP - the shear viscosity over entropy ratio $\eta / s$ and the ratio of the electric conductivity to the temperature $\sigma_{Q} / T$ - at finite temperature and chemical potential. Our consideration is based on the framework of an effective relativistic Boltzmann equation in the relaxation-time approximation, where properties of the QGP matter such as the EoS, the interaction cross sections and constituent quark masses are described by the extended Polyakov Nambu-Jona-Lasinio (PNJL) model with a critical endpoint $(\mathrm{CEP})$ located at $\left(T^{C E P}, \mu_{q}^{C E P}\right)=(0.11,0.32)$ $\mathrm{GeV}$ [175]. The framework of the PNJL model allows to evaluate the transport properties of the QGP near the chiral phase transition at moderate and high $\mu_{B}$. Here we denote the quark chemical potential as (for the light quarks) $\mu_{q}=\mu_{l}=\mu_{B} / 3$ while for the strange quarks we take $\mu_{s}=0$.

We compare the PNJL transport coefficients with the results presented in Chapter 2 ( cf. Refs. [1,2]), where they have been calculated within the DQPM [45, 46] at moderate values of the baryon chemical potential, $\mu_{B} \leq 0.5 \mathrm{GeV}$. It is important to note that both models are based on rather different ideas: The DQPM is an effective model for the description of non-perturbative (strongly interacting) QCD based on the lQCD EoS. The degrees of freedom of the DQPM are strongly interacting dynamical quasiparticles - quarks and gluons - with broad spectral functions, whose 'thermal' masses and widths increase with temperature, while the degrees of freedom of the PNJL are quarks whose masses approach the bare mass with increasing temperature and the chiral condensate disappears. Moreover, in the PNJL the mesons still exist above the Mott transition temperature as resonances. Thus, in the EoS the energy density is shared between the quarks, mesons and the Polyakov loop potential. We explore how the nature of the degrees of freedom affects the transport properties of the QGP. Moreover, we study the influence of a CEP and of a 1st order phase transition at high baryon chemical potential. For the evaluation of the relaxation time we use two different methods: a) the 'averaged transition rate' defined via the thermal averaged quark-quark and quark-antiquark PNJL cross sections and b) the 'weighted' thermal averaged quark-quark and quark-antiquark PNJL cross sections ${ }^{4}$. We discuss the uncertainties related to the theoretical methods based on the RTA.

$4 \quad$ Here under 'thermal-averaged' cross sections we mean the the interaction cross section averaged over the thermal (anti-)quark distribution function 
Part of the results presented below have been included in publications $[4,175] .^{5}$

\subsection{Basic ideas of the Nambu-Jona Lasinio model}

The Nambu-Jona Lasinio model [176] was proposed even before the theory of strong interactions, QCD, was formulated. The authors considered the possibility that nucleons generate mass due to the dynamical breaking of chiral symmetry and the formation of pions as bound states of two nucleons. Although, at the time of the formulation of this model the existence of quarks and gluons was not yet known, the authors noted that pions are not the most fundamental particles involved in the processes of the strong interaction, but are similar to collective excitations of atoms in the crystal lattice - phonons. The scheme of reasoning for the model goes back to the works on the nature of superconductivity by Bardeen, Cooper, and Schrieffer (BCS) [177], and Bogolyubov [178], where a mass gap is formed between the excited states and the ground state of the superconductor due to an attraction between electrons and the formation of compound particles obeying the BoseEinstein statistics - the so-called Cooper pairs - which are correlated pairs of electrons with oppositely directed spins.

We start with the main idea of the model, which is to consider that the gluons exchanged between quarks/antiquarks have an effective mass. This mass is supposed to be much larger than momenta of the gluons such that it can be neglected in the gluon propagators. Consequently, the propagator of a gluon is a constant and reduced to a simple effective factor. This implies that gluons do not presented in the Nambu and Jona-Lasinio model as dynamic degrees of freedom. The approximation also leads to the absence of confinement in a pure NJL description. Nevertheless, the approximation allows to simplify the calculations. The associated Lagrangian of the NJL model for $N_{f}=3$ at finite $T$ and $\mu_{f}$ reads

$$
\begin{aligned}
L_{N J L} & =\sum_{f=u, d, s} \bar{\psi}_{f}\left(i D-m_{0 f}+\mu_{f} \gamma_{0}\right) \psi_{f} \\
& +G_{s} \sum_{a} \sum_{i j k l}\left[\left(\bar{\psi}_{i} \tau_{i j}^{a} \psi_{j}\right)\left(\bar{\psi}_{k} \tau_{k l}^{a} \psi_{l}\right)+\left(\bar{\psi}_{i} i \gamma_{5} \tau_{i j}^{a} \psi_{j}\right)\left(\bar{\psi}_{k} i \gamma_{5} \tau_{k l}^{a} \psi_{l}\right)\right] \\
& -K \operatorname{det}_{i j}\left[\bar{\psi}_{i}\left(\mathbb{I}-\gamma_{5}\right) \psi_{j}\right]-K \operatorname{det}_{i j}\left[\bar{\psi}_{i}\left(\mathbb{I}+\gamma_{5}\right) \psi_{j}\right] .
\end{aligned}
$$

Here $i, j, k, l=1,2,3$ are the flavor indices and $\tau^{a}(a=1, \ldots, 8)$ are the $N_{f}=3$ flavor generators with the normalization

$$
\operatorname{Tr}_{f}\left(\tau^{a} \tau^{b}\right)=2 \delta^{a b}
$$

with $\operatorname{Tr}_{f}$ denoting the trace in the flavor space, $m_{0 i}$ stands for the bare quark masses, we consider $m_{0 u}=m_{0 d}$, and $\mu_{i}$ for their chemical potential. The first term corresponds to the

5 This work was done in collaboration with David Fuseau (SUBATECH, Nantes), who provided the PNJL results for quark masses, Polyakov loops, entropy density, and elastic cross-sections. Programs for the evaluation of the relaxation times and transport coefficients and plotting routines have been developed by the author of the present thesis. 
Lagrangian of free spin 1/2 particle, the second term describes the interactions and includes scalar and pseudo-scalar 4-point interactions with the corresponding coupling constant $G_{s}$. The last term is the so-called 't Hooft Lagrangian that breaks explicitly the pseudo-scalar symmetry $U_{A}(1) . K$ is a coupling constant, which is fixed by the value of $m_{\eta^{\prime}}-m_{\eta}$, and $\mathbb{I}$ is the identity matrix in Dirac space. This Lagrangian obeys the symmetries of thestrong interaction and conserves the currents. The NJL model is nonrenormalizable, and consequently, the integrals - essential for the evaluation of the physical quantities- diverge. Therefore a regularization has to be performed. There are various regularization schemes $[179,180]$, which are a three dimensional $(3 D)$ momentum cutoff, four-dimensional $(4 D)$ momentum cutoff, Pauli-Villars (PV) regularization, proper-time (PT) regularization, and a dimensional regularization (DR). Here we consider the most popular $3 D$ cutoff scheme.

\subsection{Polyakov extended Nambu-Jona Lasinio model}

In order to add gluons to the Nambu-Jona Lasinio model it was proposed to include thermal gluons on the level of a mean field by means of the Polaykov loops [181-184], which are the order parameter in case of pure glue $\mathrm{SU}(3)$ theory. We recall that, the Polyakov line and the Polyakov loop are, respectively, defined as

$$
L(\mathbf{x})=\mathcal{P} \exp \left(i \int_{0}^{1 / T} d \tau A_{4}(\tau, \mathbf{x})\right)
$$

and

$$
\Phi(\mathbf{x})=\frac{1}{N_{c}} \operatorname{Tr}_{c} L(\mathbf{x}),
$$

where $\mathcal{P}$ is the path-integral ordering operator and the trace $\operatorname{Tr}_{c}$ is taken in the color space. To account for deconfinement effects via the Polyakov loop, an effective potential $\mathcal{U}(T, \Phi, \bar{\Phi})$ is added to the effective NJL Lagrangian. $\mathcal{U}(T, \Phi, \bar{\Phi})$ is a function of the Polyakov loop and its complex conjugate. The Lagrangian of the PNJL model [181-185] for (color neutral) pseudoscalar and scalar interactions (neglecting the vector and axial-vector vertices for simplicity) is

$$
\begin{aligned}
L_{P N J L}= & \sum_{i} \bar{\psi}_{i}\left(i D-m_{0 i}+\mu_{i} \gamma_{0}\right) \psi_{i} \\
+ & G \sum_{a} \sum_{i j k l}\left[\left(\bar{\psi}_{i} i \gamma_{5} \tau_{i j}^{a} \psi_{j}\right)\left(\bar{\psi}_{k} i \gamma_{5} \tau_{k l}^{a} \psi_{l}\right)\right. \\
& \left.+\left(\bar{\psi}_{i} \tau_{i j}^{a} \psi_{j}\right)\left(\bar{\psi}_{k} \tau_{k l}^{a} \psi_{l}\right)\right] \\
- & K\left[\operatorname{det}_{i j}\left[\bar{\psi}_{i}\left(\mathbb{I}-\gamma_{5}\right) \psi_{j}\right]+\operatorname{det}_{i j}\left[\bar{\psi}_{i}\left(\mathbb{I}+\gamma_{5}\right) \psi_{j}\right]\right]-\mathcal{U}(T ; \Phi, \bar{\Phi})
\end{aligned}
$$

Here the covariant derivative in the Polyakov gauge reads $D^{\mu}=\partial^{\mu}-i \delta^{\mu 0} A^{0}$, with $A^{0}=$ $-i A_{4}$ being the temporal component of the gluon field in Euclidean space (we denote $\left.A^{\mu}=g_{s} A_{a}^{\mu} T_{a}\right)$. The coupling constant for the scalar and pseudoscalar interaction $G$ is 
fixed e.g. by the pion mass in vacuum. The model parameters of the PNJL are displayed in Table 2.

\begin{tabular}{|c|c|c|c|c|}
\hline$m_{u}^{0}[\mathrm{GeV}]$ & $m_{s}^{0}[\mathrm{GeV}]$ & $\mathrm{G}$ & $\mathrm{K}$ & $\Lambda[\mathrm{GeV}]$ \\
\hline 0.005 & 0.134 & $\frac{2.3}{\Lambda^{2}}$ & $\frac{11 .}{\Lambda^{5}}$ & 0.569 \\
\hline
\end{tabular}

Table 2: Parameters of the PNJL model considered in Refs. [5, 175].

The value of the potential $\mathcal{U}$ for the expectation values $\langle\Phi\rangle(T),\langle\bar{\Phi}\rangle(T)$, gives the pressure of the gluons in the Yang-Mills (YM) theory, corresponding to QCD for infinitely heavy quarks. The comparison with lattice gauge calculations for pure YM serves therefore as a guideline for the parametrisation of the effective potential $U(T)$,

$$
-P(T)=\mathcal{U}(T,\langle\Phi\rangle(T),\langle\bar{\Phi}\rangle(T))
$$

The effective potential $U(T, \phi=\langle\Phi\rangle(T), \bar{\phi}=\langle\bar{\Phi}\rangle(T))$ is parametrised following Ref. [175]:

$$
\frac{U(\phi, \bar{\phi}, T)}{T^{4}}=-\frac{b_{2}(T)}{2} \bar{\phi} \phi-\frac{b_{3}}{6}\left(\bar{\phi}^{3}+\phi^{3}\right)+\frac{b_{4}}{4}(\bar{\phi} \phi)^{2}
$$

with the parameters

$$
b_{2}(T)=a_{0}+\frac{a_{1}}{1+\tau}+\frac{a_{2}}{(1+\tau)^{2}}+\frac{a_{3}}{(1+\tau)^{3}}
$$

where

$$
t_{\text {phen }}=0.57 \frac{T-T_{\text {phen }}(T)}{T_{\text {phen }}(T)}
$$

and

$$
T_{p h e n}(T)=a+b T+c T^{2}+d T^{3}+e \frac{1}{T} .
$$

All the coefficients of the parametrisation are listed in Table 3.

\begin{tabular}{|c|c|c|c|c|c|c|c|c|c|c|}
\hline$a_{0}$ & $a_{1}$ & $a_{2}$ & $a_{3}$ & $b_{3}$ & $b_{4}$ & $\mathrm{a}$ & $\mathrm{b}$ & $\mathrm{c}$ & $\mathrm{d}$ & $\mathrm{e}$ \\
\hline 6.75 & -1.95 & 2.625 & -7.44 & 0.75 & 7.5 & 0.082 & 0.36 & 0.72 & -1.6 & -0.0002 \\
\hline
\end{tabular}

Table 3: Parameters of the effective potential $\mathrm{U}(\phi, \bar{\phi}, \mathrm{T})$ used in this work.

\subsection{Quarks and mesons in the PNJL model}

In order to calculate cross sections, the masses of the quarks and the propagators of the exchanged mesons have to be known. The mass of the quarks is determined by solving the gap equations [184] together with a minimisation of the grand potential $\Omega_{P N J L}$ with 

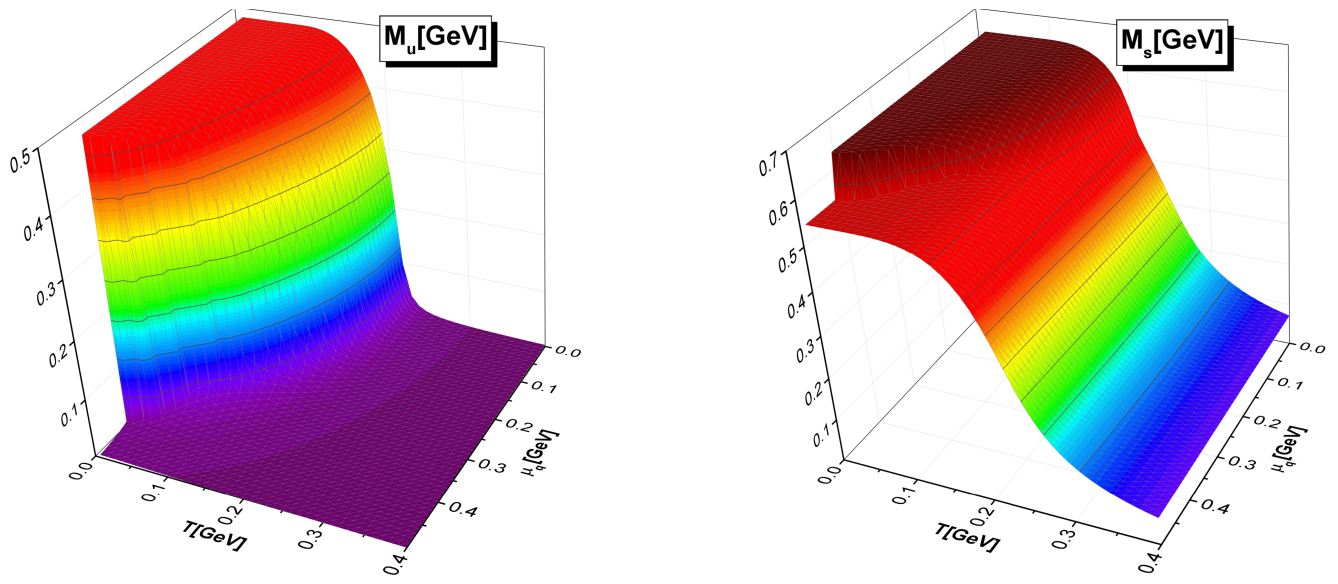

Figure 4.1: The effective masses of light (left) and strange (right) quarks as a function of temperature $T$ and quark chemical potential $\mu_{q}$ from the PNJL model. The figure is adopted from [5].

respect to the Polyakov loop expectation value $\phi$ and $\bar{\phi}$ :

$$
\begin{gathered}
\frac{\partial \Omega_{P N J L}}{\partial \phi}=0, \\
\frac{\partial \Omega_{P N J L}}{\partial \bar{\phi}}=0, \\
m_{q}=m_{q 0}-4 G\left\langle\bar{\psi}_{q} \psi_{q}\right\rangle+2 K\left\langle\bar{\psi}_{q} \psi_{q}\right\rangle\left\langle\bar{\psi}_{s} \psi_{s}\right\rangle, \\
m_{s}=m_{s 0}-4 G\left\langle\bar{\psi}_{s} \psi_{s}\right\rangle+2 K\left\langle\bar{\psi}_{q} \psi_{q}\right\rangle\left\langle\bar{\psi}_{q} \psi_{q}\right\rangle .
\end{gathered}
$$

The thermodynamic potential for the PNJL model reads,

$$
\begin{aligned}
& \Omega_{P N J L}\left(T, \mu_{i}, \Phi, \bar{\Phi}\right)= \\
& 2 G \sum_{i}\left\langle\bar{\psi}_{i} \psi_{i}\right\rangle^{2}-4 K \prod_{i}\left\langle\bar{\psi}_{i} \psi_{i}\right\rangle-2 N_{c} \sum_{i} \int_{0}^{\Lambda} \frac{d^{3} p}{(2 \pi)^{3}} E_{i} \\
& -2 T N_{c} \sum_{i} \int_{0}^{\infty} \frac{d^{3} p}{(2 \pi)^{3}}\left(\frac{1}{N_{C}} \log \left[1+3\left(\Phi+\bar{\Phi} e^{-\left(E_{i}-\mu_{i}\right) / T}\right) e^{-\left(E_{i}-\mu_{i}\right) / T}\right]\right. \\
& \left.+\frac{1}{N_{c}} \log \left[1+3\left(\bar{\Phi}+\Phi e^{-\left(E_{i}+\mu_{i}\right) / T}\right) e^{-\left(E_{i}+\mu_{i}\right) / T}\right]\right) \\
& +U_{P N J L},
\end{aligned}
$$

with $E_{i}=\sqrt{p^{2}+m_{i}^{2}}$ denoting the on-shell energies.

Figure 4.1 displays the PNJL effective masses of the $u$ and $s$ quarks as a function of the chemical potential $\mu_{q}$ and $T$. One can see that a smooth crossover occurs for small chemical potentials. The $T$-dependence of the effective masses in the PNJL model is governed by the chiral condensate $\langle\bar{\psi} \psi\rangle$, which drops from its maximal value in the hadronic phase down to zero in the QGP phase.

The propagators of the mesons are build by resummation of the quark-antiquark loops 
within the random-phase approximation (RPA), leading to the amplitude [179]:

$$
D^{a}=\frac{2 i g_{m}}{1-2 g_{m} \Pi_{f f^{\prime}}^{ \pm}\left(k_{0}, \vec{k}\right)}
$$

where $a=S, P S$ refers to the scalar and pseudoscalar exchanging mesons and the indexes $f, f^{\prime}$ denote the (anti-)quark flavors. The mass of the exchanged meson is given by the pole of the propagator. Here $g_{m}$ denotes the coupling constant [186] and $\Pi_{f f^{\prime}}^{ \pm}\left(k_{0}, \vec{k}\right)$ is the quark-antiquark polarisation function given by

$$
\begin{aligned}
& \Pi_{f f^{\prime}}^{ \pm}\left(k_{0}, \vec{k}\right)=-\frac{N_{c}}{4 \pi^{2}}\left[A_{0}\left(m_{f}, \mu_{f}, T, \Lambda\right)+A_{0}\left(m_{f^{\prime}}, \mu_{f^{\prime}}, T, \Lambda\right)\right. \\
& \left.+\left[\left(m_{f} \pm m_{f^{\prime}}\right)^{2}-\left(k_{0}+\mu_{f}-\mu_{f^{\prime}}\right)^{2}+\vec{k}^{2}\right] \times B_{0}\left(\vec{k}, m, \mu, m^{\prime}, \mu^{\prime}, k_{0}, T, \Lambda\right)\right],
\end{aligned}
$$

where "+" stands for the scalar and "-" for the pseudoscalar mesons. The one-fermion loop $A_{0}$ is separated into a vacuum part and a thermal part, the latter being integrated up to infinity:

$$
A_{0}\left(m_{f}, \mu_{f}, T, \Lambda\right)=-4\left[\int_{0}^{\infty} d p \frac{p^{2}}{E_{f}}\left(-f_{f}\left(E_{f}, T, \mu_{f}\right)-f_{\bar{f}}\left(E_{f}, T, \mu_{f}\right)\right)+\int_{0}^{\Lambda} \frac{p^{2} d p}{E_{f}}\right] .
$$

Here $E_{f}=\sqrt{p^{2}+m_{f}^{2}}$ and the Fermi-Dirac distribution functions $f_{f(\bar{f})}\left(E_{f}, T, \mu_{f}\right)$ $=f_{i}^{(0)}\left(E_{i}, T, \mu_{i}\right)$ are defined by Eq. (58).

The two-Fermion loop $B_{0}$ is defined as [187]

$$
\begin{aligned}
& B_{0}\left(\vec{p}, m_{f}, \mu_{f}, m_{f^{\prime}}, \mu_{f^{\prime}}, i \nu_{m}, T, \Lambda\right)=16 \pi^{2} T \sum_{n} \exp \left(i \omega_{n} \eta\right) \\
& \times \int_{|q|<\Lambda} \frac{d^{3} q}{(2 \pi)^{3}} \frac{1}{\left[\left(i \omega_{n}+\mu_{f}\right)^{2}-E_{f}^{2}\right]} \frac{1}{\left[\left(i \omega_{n}-i \nu_{m}+\mu_{f}^{\prime}\right)^{2}-E_{f^{\prime}}^{2}\right]}
\end{aligned}
$$

with $E_{f}=\sqrt{\vec{q}^{2}+m_{f}^{2}}, E_{f^{\prime}}=\sqrt{(\vec{q}-\vec{p})^{2}+m_{f^{\prime}}}$. The details of the calculations of $B_{0}$ can be found in Ref. [187]. We have $g_{m}^{ \pm}=G \pm \frac{1}{2} K S^{s}$ for the pion $(+)$ and its scalar partner $(-)$ and $g_{m}^{ \pm}=G \pm \frac{1}{2} K S^{u}$ for the kaon $(+)$ and its scalar partner (-).

For the $\eta$ mesons, the propagators are more complicated because of the mixing terms [188], [179]:

$$
\begin{gathered}
D=2 \frac{\operatorname{det} K}{M_{00} M_{88}-M_{08}^{2}}\left(\begin{array}{cc}
M_{00} & M_{08} \\
M_{80} & M_{88}
\end{array}\right) \\
D=\frac{4}{3} \frac{\operatorname{det} K}{M_{00} M_{88}-M_{08}^{2}}\left(M_{00} \bar{\psi} \lambda_{0} \psi \cdot \bar{\psi}^{\prime} \lambda_{0} \psi^{\prime}+M_{08} \bar{\psi} \lambda_{0} \psi \cdot \bar{\psi}^{\prime} \lambda_{8} \psi^{\prime}\right. \\
\left.+M_{80} \bar{\psi} \lambda_{8} \psi \cdot \bar{\psi}^{\prime} \lambda_{0} \psi^{\prime} M_{88} \bar{\psi} \lambda_{8} \psi \cdot \bar{\psi}^{\prime} \lambda_{8} \psi^{\prime}\right)
\end{gathered}
$$




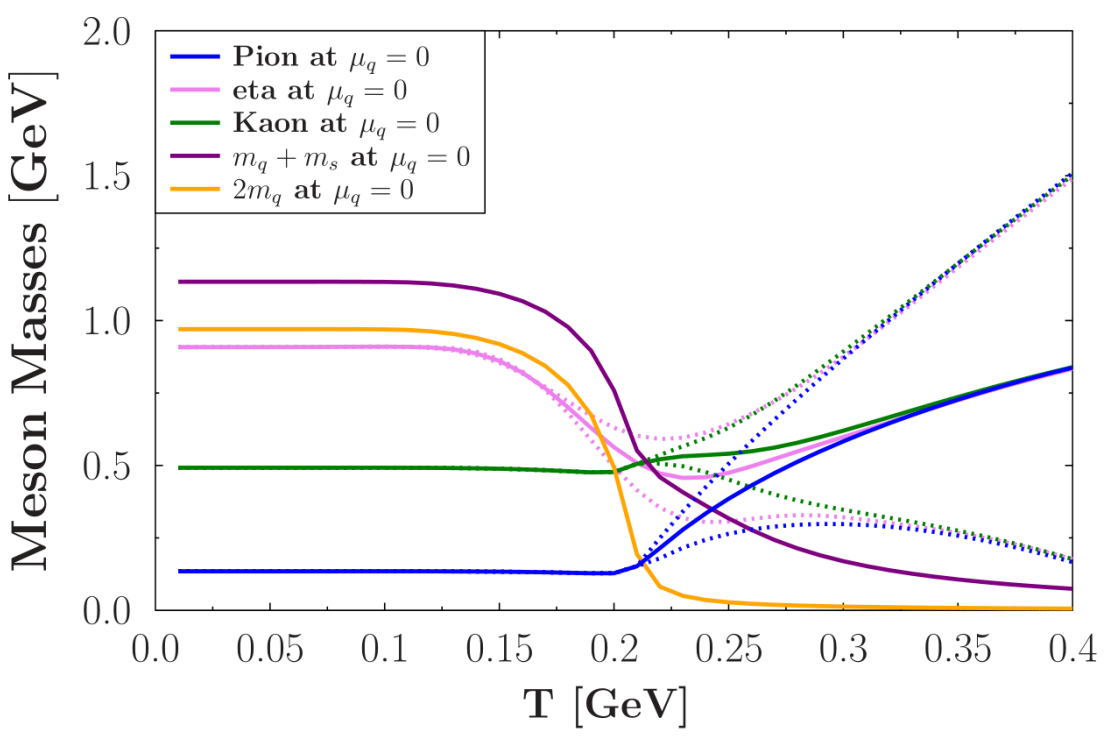

Figure 4.2: Meson masses (pion, eta, kaon) as well as double quark masses $2 m_{q}$ and $m_{s}+m_{q}$ from the $N_{f}=3$ PNJL model at $\mu_{q}=0$ as a function of temperature. The dotted lines indicate the $m_{\text {pole }} \pm \Gamma$, where $\Gamma$ is the imaginary part of the complex pole of the meson propagators and $m_{\text {pole }}$ is its real part (indicated by solid lines). The figure is taken from [5].

with

$$
\begin{aligned}
M_{00} & =K_{0}^{+}-\frac{4}{3} \operatorname{det} K\left(\Pi_{u \bar{u}}+2 \Pi_{s \bar{s}}\right) \\
M_{08} & =K_{08}^{+}-\frac{4}{3} \sqrt{2} \operatorname{det} K\left(\Pi_{u \bar{u}}-\Pi_{s \bar{s}}\right) \\
M_{88} & =K_{8}^{+}-\frac{4}{3} \operatorname{det} K\left(2 \Pi_{u \bar{u}}+\Pi_{s \bar{s}}\right) \\
\operatorname{det} K & =K_{0}^{+} K_{8}^{+}-K_{08}^{2}
\end{aligned}
$$

and

$$
\begin{aligned}
& K_{0}^{ \pm}=G \mp \frac{1}{3} K\left(2 G^{u}+G^{s}\right) \\
& K_{08}^{ \pm}= \pm \frac{1}{6} \sqrt{2} K\left(G^{u}-G^{s}\right) \\
& K_{8}^{ \pm}=G \pm \frac{1}{6} K\left(4 G^{u}-G^{s}\right),
\end{aligned}
$$

where $G^{i}$ is the spinor trace of the propagator $S^{i}(x, x)$ :

$$
G^{i}=N_{c} i \operatorname{Tr}\left[S^{i}(x, x)\right]=-\frac{N_{C}}{4 \pi^{2}} m_{i} A_{0}\left(m_{i}, \mu_{i}, T, \Lambda\right) .
$$

The masses of the pseudoscalar mesons (pion, eta, kaon) in comparison to the doubled quark masses $2 m_{q}$ and $m_{q}+m_{s}$ at $\mu_{q}=0$ are presented in Fig. 4.2. While in the PNJL the quark masses drop with increasing temperature to their bare values due to the 
disappearance of the chiral condensate in the vicinity of the phase transition, the meson masses increase with temperature. In the PNJL mesons become unstable above the Mott temperature, $T>T_{M \pi}$, where the total mass of the constituent quarks equals the meson mass. Above $T_{M \pi}$ the mesons can decay into a $q \bar{q}$ pair and the pole of the meson propagator becomes complex above $T_{M \pi}$. The dotted lines in Fig. 4.2 correspond to $m_{\text {pole }} \pm \Gamma$, where $\Gamma$ is the imaginary part of the complex pole of the meson propagator (which could be associated with the decay width) and $m_{\text {pole }}$ is its real part, indicated by solid lines. It is important to note here, that contrary to mesons, the quarks in the PNJL stay on-shell.

\subsection{Thermodynamic properties of the PNJL model}

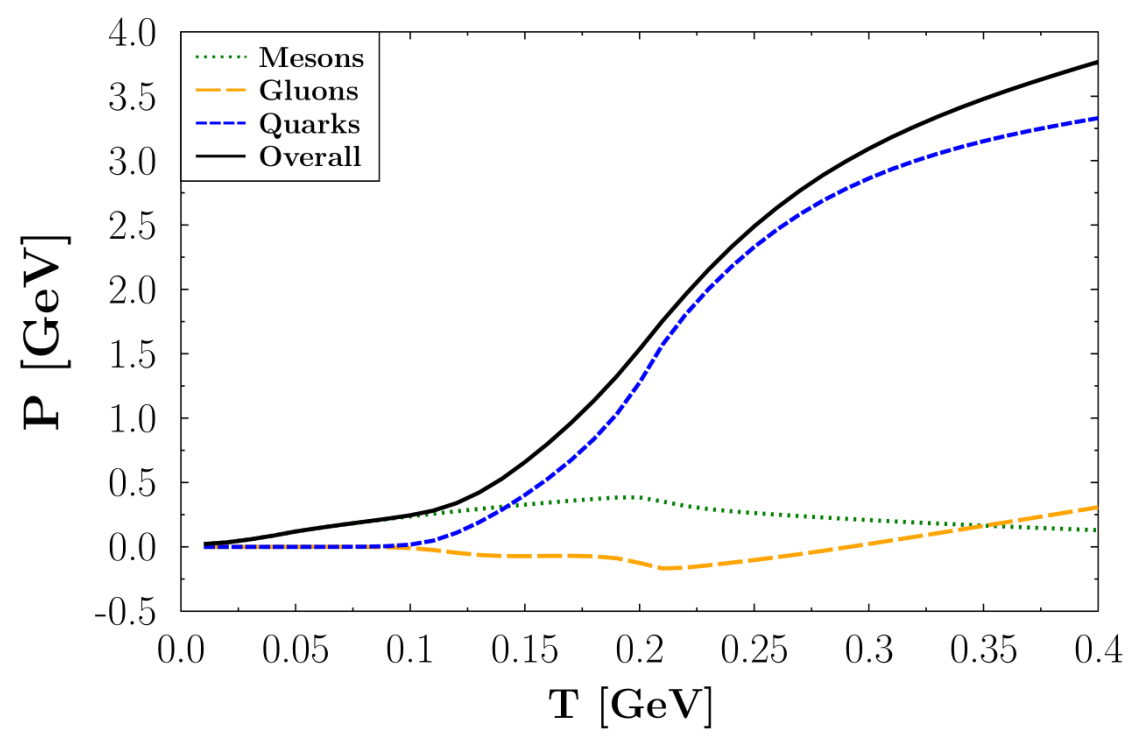

Figure 4.3: Meson, gluon, and quarks contributions to the total pressure as well as the total pressure (solid black line) at $\mu_{q}=0$ as a function of the temperature. The figure is taken from [5].

The starting point for the calculations of thermodynamic quantities is the thermodynamic potential of the system, which (in case of the grand canonical ensemble) is related to the pressure by $\Omega=-P / V=-p$. All other thermodynamic observables then are obtained from the total pressure. Here we discuss basic thermodynamic observables of the improved PNJL model advanced in Ref. [175] which matches the 1QCD results obtained by the HotQCD collaboration [72]. It is important to note that the improved PNJL model differs from the standard PNJL model in two aspects:

- The grand potential includes next-to-leading order contributions in $N_{c}$ and contains therefore contributions from mesons.

- A temperature-dependent rescaling of the $T_{0}$ parameters of the standard Polyakov effective potential, see Eq. (128) and Table 3, in order to phenomenologically reproduce the quark gluon interactions in the medium. 


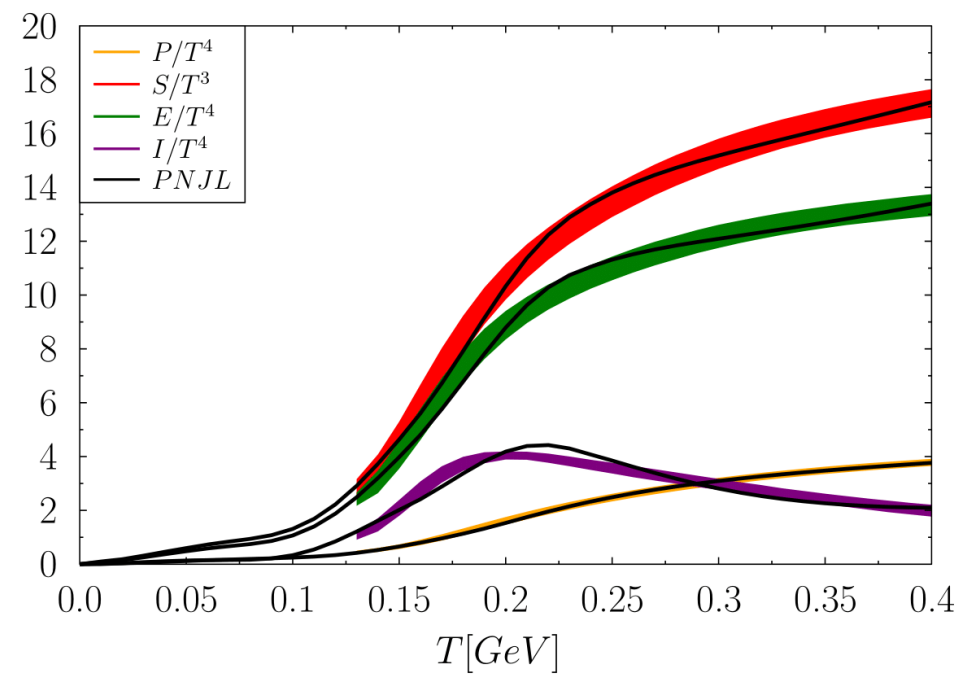

Figure 4.4: Basic thermodynamic observables from the improved PNJL model (solid lines): scaled pressure, entropy density, energy density and interaction measure as a function of temperature $T$ at $\mu_{q}=0$ in comparison to the lattice QCD results [72] (colored bands). The figure is taken from [5].

In next-to-leading order the grand potential contains an additional term, $\Omega_{M}$, caused by the diagrams of the order $\mathcal{O}\left(N_{c}=1\right)$ in the $N_{c}$ expansion. This term is given by $[175,189]$ :

$$
\begin{aligned}
& \Omega_{M}=-\frac{g_{M}}{8 \pi^{3}} \int d p p^{2} \int \frac{d s}{\sqrt{s+\vec{k}^{2}}}\left[\frac{1}{\exp \left(\left(\sqrt{s+\vec{k}^{2}}-\mu\right) / T-1\right)}\right. \\
& \left.+\frac{1}{\exp \left(\left(\sqrt{s+\vec{k}^{2}}+\mu\right) / T-1\right)}\right] \delta\left(\sqrt{s}, T, \mu_{M}\right) .
\end{aligned}
$$

Here $g_{M}$ is the degeneracy of the meson and $\delta\left(\sqrt{s}, T, \mu_{M}\right)$ is a phase shift defined by

$$
\delta\left(\sqrt{s}, T, \mu_{M}\right)=-\operatorname{Arg}\left[1-2 K \Pi\left(\omega-\mu_{M}+i \epsilon, \vec{k}\right)\right],
$$

where $\Pi$ is the polarization function of the meson. $\Omega_{M}$ represents a mesonic pressure that dominates at low temperature and is non negligible around $T_{C}$ as seen from Fig. 4.3.

One can see from Fig. 4.4 that basic thermodynamic observables from the PNJL model are in a good agreement with lattice results of the HotQCD collaboration taken from Ref. [72] at vanishing chemical potential.

Furthermore, the important limit of large chemical potentials and low temperatures, is accessible for the perturbative calculations in the PNJL model. The resulting PNJL pressure at approximately zero temperature is shown in comparison to the perturbative results from Ref. [190] (accurate to $O\left(g^{5}\right)$ in the gauge coupling) in Fig. 4.5. 


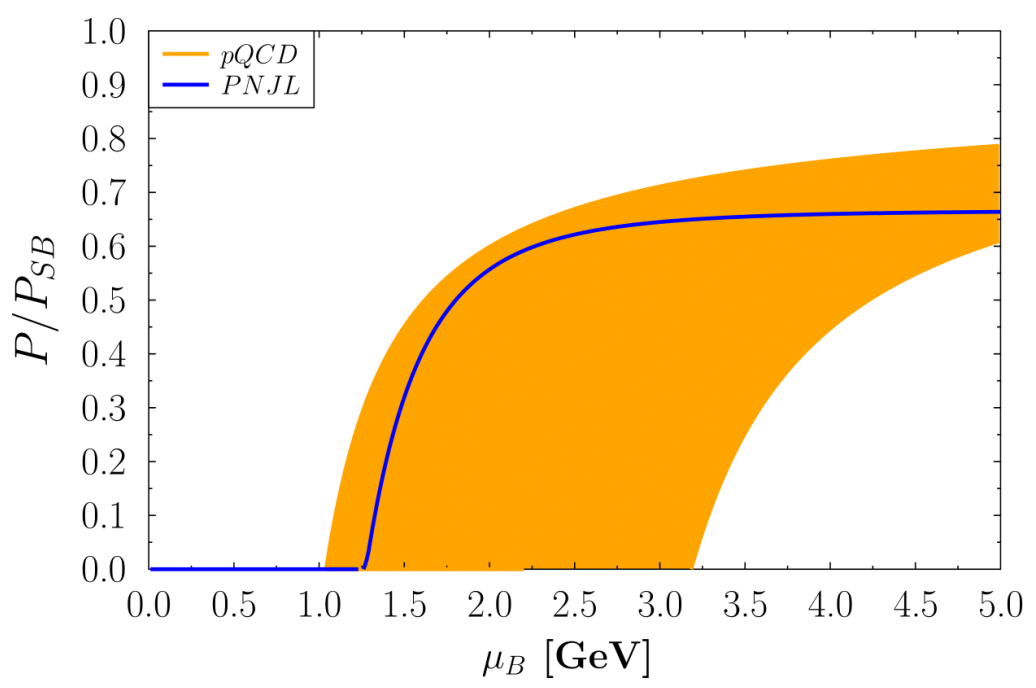

Figure 4.5: Ratio of quark pressure to the pressure in the Stefan-Boltzmann limit as a function of $\mu_{B}=3 \mu_{q}$ for a temperature of $T=0.001 \mathrm{GeV}$. We compare the resulting ratio for the PNJL approach (blue line) to the perturbative results from Ref. [190] (orange area). The figure is taken from [5].

\subsection{Phase diagram of the $N_{f}=3$ PNJL model}

It is expected that the order of the phase transition of QCD matter between the hadronic phase and the QGP phase can change with increasing baryon chemical potential. While at small baryon chemical potentials the IQCD predicts that the transition is a crossover [60,72], at higher baryon chemical potentials the existence of the CEP and a 1st-order phase transition and it's influence on the properties of the QGP matter should be addressed. In the PNJL model a possible first-order phase transitions at high quark or baryon chemical potential is considered. It terminates at the CEP, located at smaller quark chemical potential, where the transition is of second-order. In the considered PNJL model the CEP is located at $\left(T^{C E P}, \mu_{q}^{C E P}\right)=(0.11,0.32) \mathrm{GeV}$. It is known that the critical line of the PNJL model generally depends on the parameters of the model [191], being usually located higher than the critical line predicted by the lQCD results [192]. The actual phase diagram of the $N_{f}=3$ PNJL model, which employed here, is depicted in Fig. 4.6. 


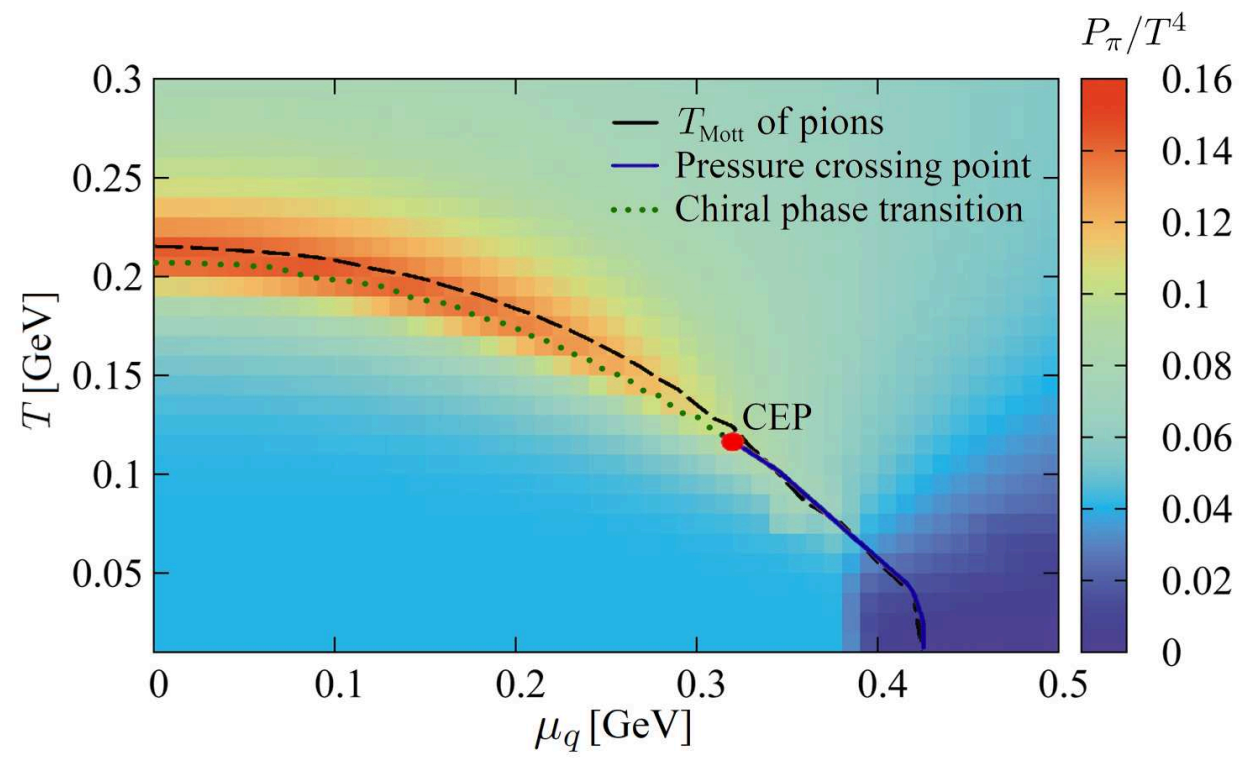

Figure 4.6: Phase diagram of QCD matter described by the $N_{f}=3$ PNJL model [175]. Scaled pion pressure $P_{\pi} / T^{4}$ in the $N_{f}=3$ PNJL model in the $\left(T, \mu_{q}\right)$ coordinates.

\subsection{Elastic quark cross-sections in the PNJL model}

The differential cross sections for the quark-quark and quark-antiquark scattering for $t-$, $u-$ channels and $s$ - channels, respectively, are given by the following expressions:

$$
\frac{d \sigma}{d t}=\frac{1}{16 \pi s_{i j}^{+} s_{i j}^{-}} \frac{1}{4 N_{c}^{2}} \sum_{s, c}\left|M_{s / u}-M_{t}\right|^{2}
$$

where we use the notation $s_{i j}^{ \pm}=s-\left(m_{i} \pm m_{j}\right)^{2}$. A detailed evaluation of the matrix elements is given in Appendix B. The total cross section in a thermal medium is obtained by integration over $t$ :

$$
\sigma_{i j \rightarrow c d}=\int_{t_{\min }}^{t^{\max }} d t \frac{d \sigma}{d t}\left(1-f_{c}^{(0)}\right)\left(1-f_{d}^{(0)}\right)
$$

where we take into account the Pauli blocking factors for the fermions due to the fact that some of the final states are already occupied by other quarks (antiquarks). The limits of the integrations are defined as,

$$
\begin{aligned}
t_{\max / \min } & =m_{i}^{2}+m_{c}^{2}-\frac{1}{2 s}\left(s+m_{i}^{2}-m_{j}^{2}\right)\left(s+m_{c}^{2}-m_{d}^{2}\right) \\
& \pm 2 \sqrt{\frac{\left(s+m_{i}^{2}-m_{j}^{2}\right)^{2}}{4 s}-m_{i}^{2}} \sqrt{\frac{\left(s+m_{c}^{2}-m_{d}^{2}\right)^{2}}{4 s}-m_{c}^{2}}
\end{aligned}
$$

where $m_{i}$ and $m_{j}$ are the masses of the particles in the entrance channel and $m_{c}$ and $m_{d}$ of those in the exit channel.

In Fig. 4.7 we present the quark-quark cross sections for $u d \rightarrow u d$ elastic scattering versus $\sqrt{s}$ at $T=190 \mathrm{MeV}$ for different $\mu_{q}=0,0.1,0.2 \mathrm{GeV}$ (top) and at $\mu_{q}=0$ for 

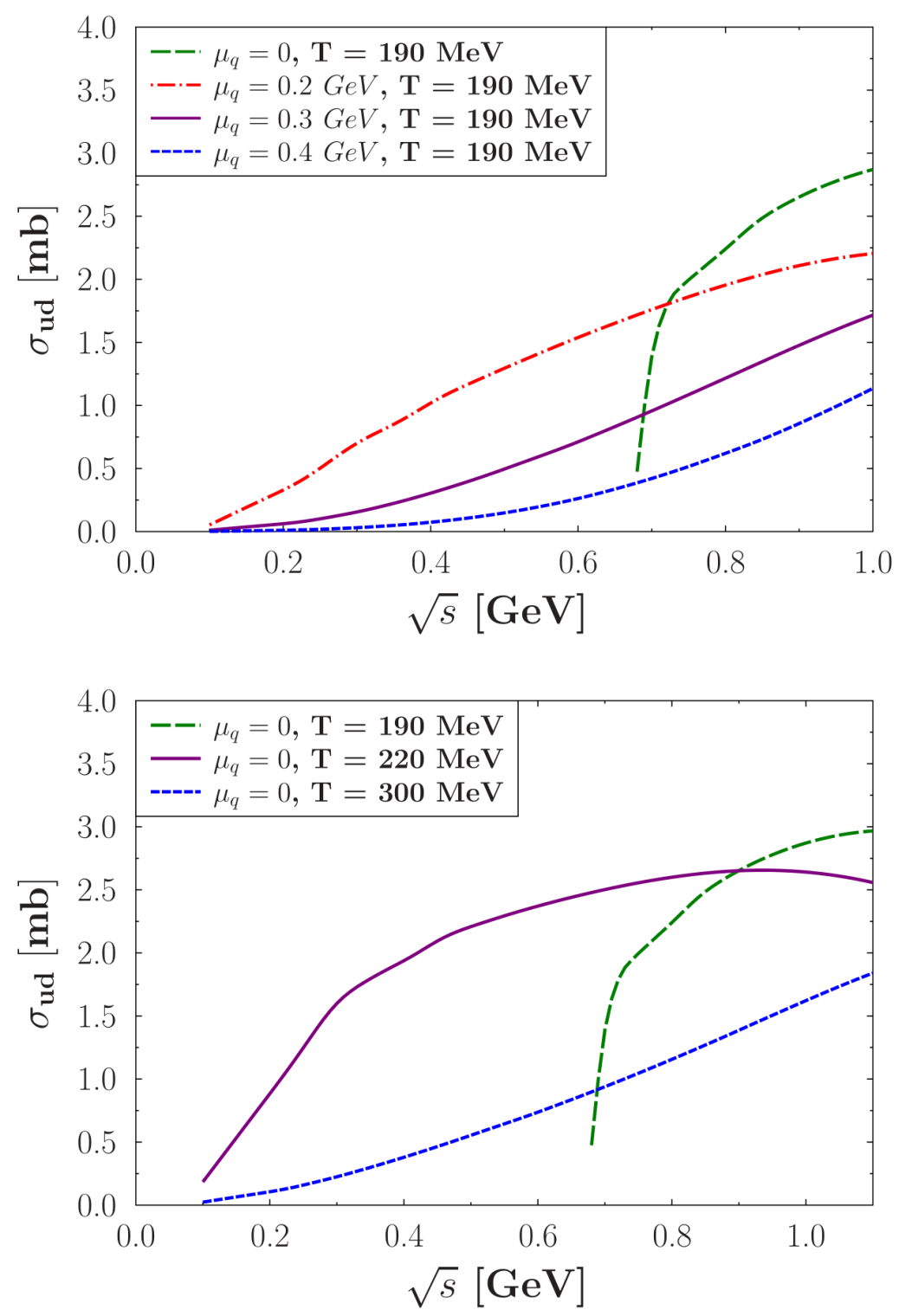

Figure 4.7: Cross section $\sigma_{u d}$ as a function of $\sqrt{s}$ at $T=190 \mathrm{MeV}$ for $\mu_{q}=0,0.1,0.2$ $\mathrm{GeV}$ (top) and at $\mu_{q}=0$ for $T=190,220$ and $300 \mathrm{MeV}$ (bottom). The figure is taken from [5].

different $T=190,220$ and $300 \mathrm{MeV}$ (bottom). As follows from Fig. 4.7 these cross sections are rather small and show a smooth behavior versus the center of mass energy. They are decreasing with increasing temperature and chemical potential as expected because the mass of the exchanged meson and its decay width increase with $T$. At low temperature and chemical potential the masses of the quarks are large and tend to vanish at large $T$ and $\mu_{q}$. Consequently, the threshold - given by $\sqrt{s}_{\text {thr }}=\operatorname{Max}\left(m_{\text {in }}^{a}+m_{\text {in }}^{b}, m_{\text {out }}^{c}+m_{\text {out }}^{d}\right)-$ is high at low $\mu_{q}$ and low $T$, as one can see from Fig. 4.7 as well.

The more interesting processes are quark-antiquark collisions. In this case the $s-$ channel allows for a resonance of the exchanged meson with the incoming quarks which leads to a large peak in the cross sections.

Fig. 4.8 (top) displays the cross section for different channels showing a resonance 

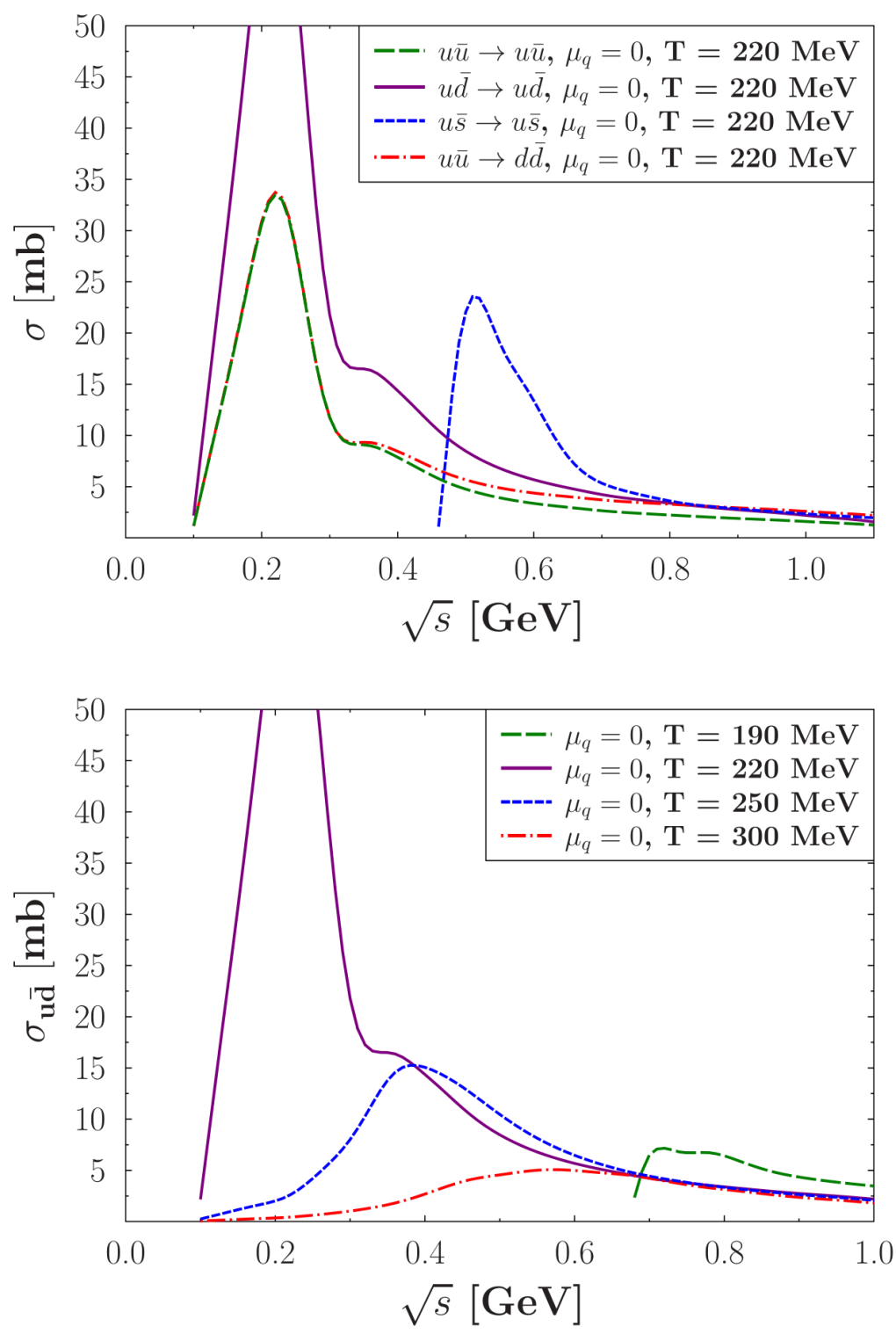

Figure 4.8: Cross sections for the $q \bar{q} \rightarrow q \bar{q}$ channels: $u \bar{u} \rightarrow u \bar{u}, u \bar{d} \rightarrow u \bar{d}, u \bar{u} \rightarrow d \bar{d}$ and $u \bar{s} \rightarrow u \bar{s}$ as a function of $\sqrt{s}$ at $T=220 \mathrm{GeV}$ and $\mu_{q}=0$ (top) and for $u \bar{d} \rightarrow u \bar{d}$ at $\mu_{q}=0$ for $T=190,220,250$ and $300 \mathrm{MeV}$ (bottom). The figure is taken from [5].

behaviour. The $u \bar{s} \rightarrow u \bar{s}$ resonance is lower than the others because the strange quark is heavier than the $\mathrm{u}$ and $\mathrm{d}$ quarks at $\mu_{q}=0$ and $T=200 \mathrm{MeV}$. The other resonances differ only by their flavour factors [193]. The $u \bar{d} \rightarrow u \bar{d}$ channel has the largest factor, $u \bar{u} \rightarrow u \bar{u}$ has a lower factor than $u \bar{u} \rightarrow d \bar{d}$ but allows for $\eta$-meson exchange which is not the case for the $u \bar{u} \rightarrow d \bar{d}$ channel.

The behavior of the $u \bar{d} \rightarrow u \bar{d}$ cross section for different temperatures is displayed in Fig. 4.8 (bottom). One can see that the resonance is shifted to the right with increasing temperature. Since the mass of the mesons increases with $T$, the cross section with the pion in the $s$-channel becomes resonant at the corresponding $\sqrt{s}$. The peak becomes lower with increasing temperature and disappears finally at large temperatures since the decay width of the pion becomes larger with increasing temperature, see Fig. 4.2. The kinematic 
threshold forbids any resonance state below the Mott temperature. This explains the flatness of the $u \bar{d} \rightarrow u \bar{d}$ cross section at $T=190 \mathrm{MeV}$.
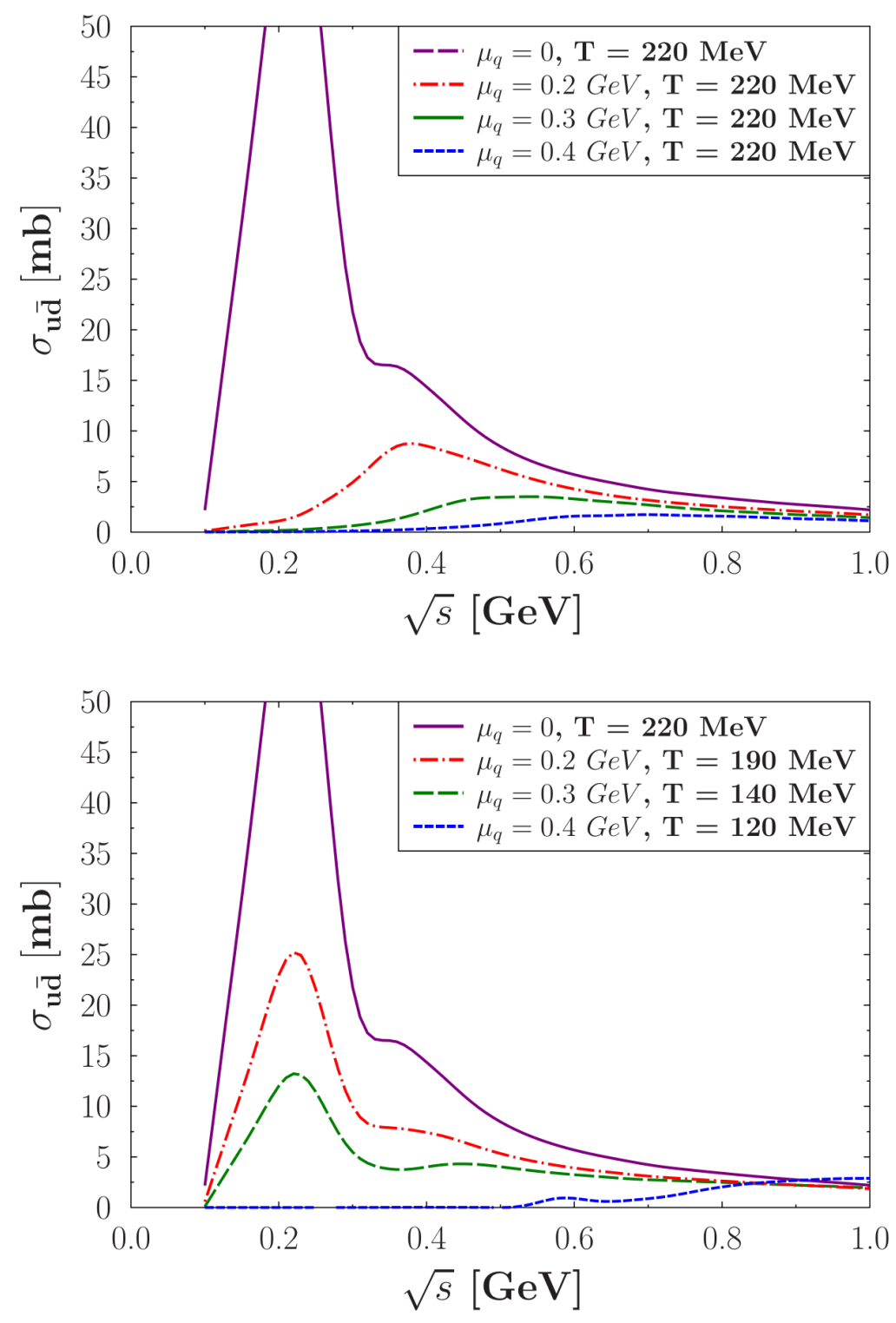

Figure 4.9: Resonant $u \bar{d}$ cross section as a function of $\sqrt{s}$ at $T=220 \mathrm{MeV}$ for $\mu_{q}=0$, 200, 300 and $400 \mathrm{MeV}$ (top) and for different combinations of $T, \mu_{q}$ (bottom). The figure is taken from [5].

The $\sqrt{s}$-dependence of the $u \bar{d}$ cross section with the resonance peak for fixed values of quark chemical potential is demonstrated in Fig. 4.9. At fixed temperature, the pion mass becomes larger with increasing quark chemical potential and the peak is shifted to a smaller temperature. For quark chemical potentials beyond the critical $\mu_{q}>\mu_{C E P}=0.32$ $\mathrm{GeV}$ the cross section is flat and no resonance behaviour is observed.

Moreover, the evaluation of the two cross sections $s \bar{s} \rightarrow u \bar{u}$ and $u \bar{u} \rightarrow s \bar{s}$ can be checked 


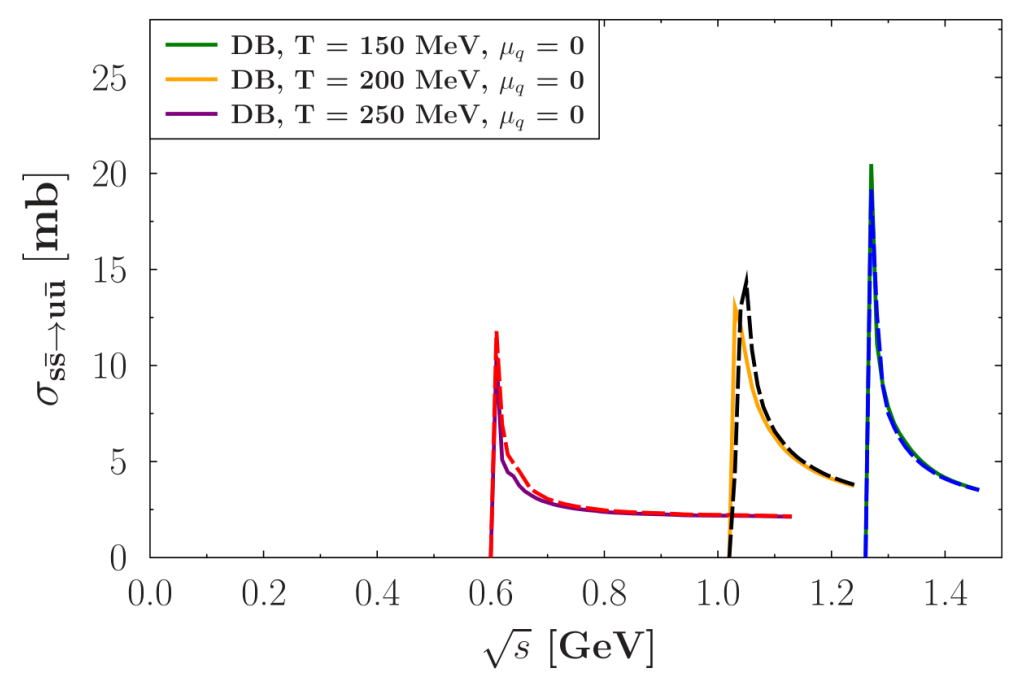

Figure 4.10: Cross section for the $s \bar{s} \rightarrow u \bar{u}$ channel calculated by the detailed balance (solid lines, DB) for $T=150,200,250 \mathrm{MeV}$ at $\mu_{q}=0$ as compared to the direct numerical calculation (dashed lines). The figure is taken from [5].

since they obey detailed balance:

$$
\sigma_{c d \rightarrow i j}(s)=\frac{p_{i j}^{2 c m}(s)}{p_{c d}^{2 c m}(s)} \sigma_{i j \rightarrow c d}(s) .
$$

Figure 4.10 displays the cross section for the $s \bar{s} \rightarrow u \bar{u}$ channel at $\mu_{q}=0$ and $T=150$, 200, $250 \mathrm{MeV}$ obtained directly (dashed lines) and by detailed balance (148) (solid lines). One can see that both calculations show a good agreement with each other within the numerical accuracy.

\subsection{Transport coefficients in the PNJL model}

The evaluation of transport coefficients is performed on the base of the relaxation time approximation of the Boltzmann equation for the quasiparticles with dynamical masses $M_{i}\left(T, \mu_{q}\right)$ described in Chapter 2 in case of the DQPM. However, one should consider some modifications due to the difference between the two models:

- In PNJL the degrees of freedom are quarks whose masses approach the bare mass when the temperature increases and the chiral condensate disappears. Moreover, in the PNJL the mesons exist above the Mott transition temperature as resonances.

- Due to the presence of the Polyakov loop potential in the thermodynamic potential modified distribution functions for quarks have to be considered. 

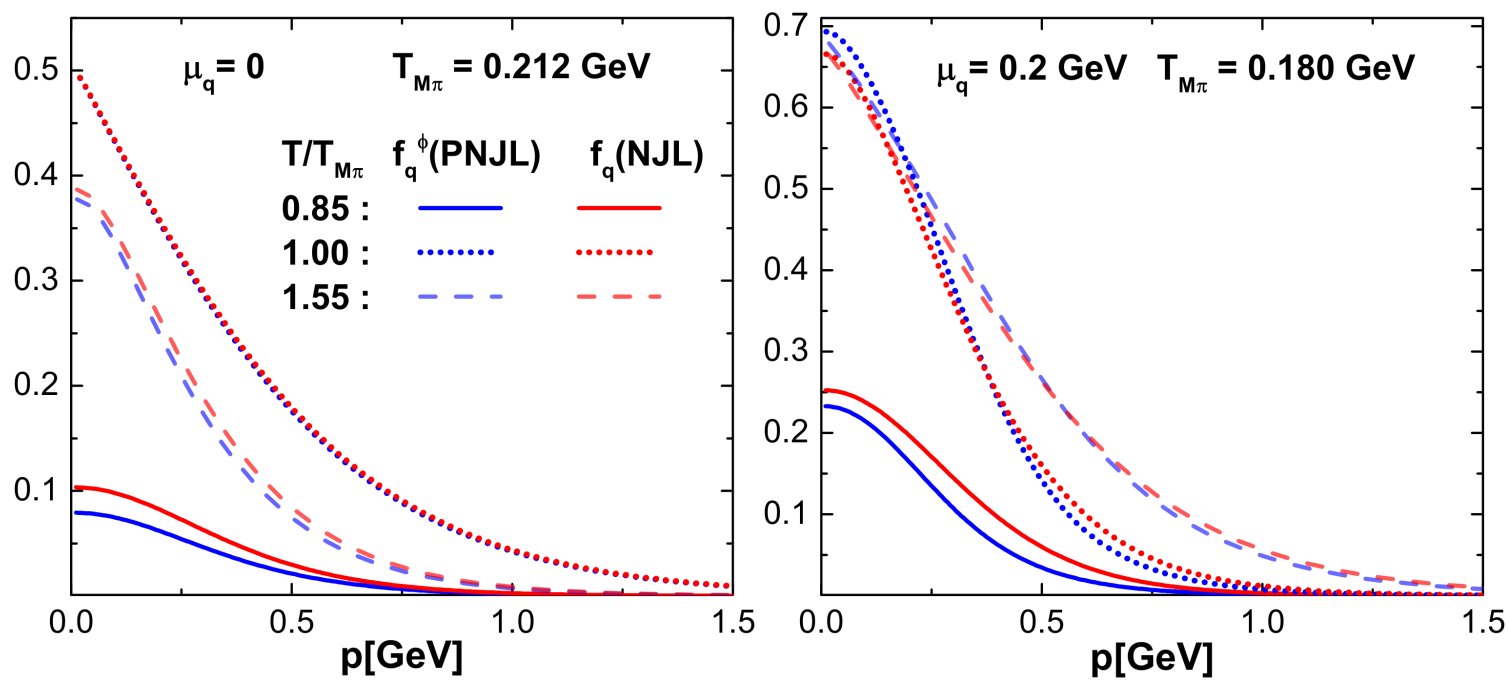

Figure 4.11: Modified (by the Polyakov loop) Fermi - Dirac distribution functions (used for the PNJL) in comparison to the Fermi - Dirac distribution functions (used for the NJL model) as a function of momentum for $\mu_{q}=0$ (left) and $\mu_{q}=0.2 \mathrm{GeV}$ (right).

The (anti-)quark density is defined as

$$
n_{i}\left(T, \mu_{q}\right)=d_{q} \int \frac{d^{3} p}{(2 \pi)^{3}} f_{i}^{\phi}
$$

where $i=u, d, s, \bar{u}, \bar{d}, \bar{s}$ and $d_{q}=2 \times N_{c}$ is the degeneracy factor for (anti-)quarks. The modified (by the Polyakov loop) Fermi - Dirac distribution function (used for the PNJL) reads,

$$
f_{i}^{\phi}=\frac{\phi e^{-\left(E_{i} \mp \mu\right) / T}+2 \bar{\phi} e^{-2\left(E_{i} \mp \mu\right) / T}+e^{-3\left(E_{i} \mp \mu\right) / T}}{1+3 \phi e^{-\left(E_{i} \mp \mu\right) / T}+3 \bar{\phi} e^{-2\left(E_{i} \mp \mu\right) / T}+e^{-3\left(E_{i} \mp \mu\right) / T}},
$$

where $i=q, \bar{q}$. The minus sign refers to quarks $(i=q)$, while the plus sign refers to antiquarks $(i=\bar{q})$. For antiquarks we have to exchange $\phi$ and $\bar{\phi}$.

In the QGP phase the modified distributions approach the standard Fermi-Dirac distributions for $\phi \rightarrow 1$, while in the hadronic phase (for $\phi \rightarrow 0$ ) we get distributions with three times the quark energy in the exponent, which can be interpreted as Fermi-Dirac distribution functions for particles with three times the quark mass. A comparison between the two distribution functions is displayed in Fig. 4.11 for different temperatures and fixed quark chemical potential.

\subsection{Quark relaxation time in the PNJL model}

The RTA is often used in the framework of effective models for an estimate of transport coefficients in the QGP phase. It is worth to note that the results of transport calculations depend not only on the EoS, which can be fitted to the LQCD results, but also (if no local equilibrium is assumed) on transport coefficients and therefore on the method of how to evaluate quark and gluon relaxation times.

In this Section we apply two different approaches for the calculation of the quark 
relaxation time, which are commonly used in the literature: 1) the so called 'averaged transition rate' defined via the thermal averaged quark-quark and quark-antiquark PNJL cross sections and 2) the 'weighted' thermal averaged quark-quark and quark-antiquark PNJL cross sections. As will be demonstrated later, the differences between both methods are quite essential and influence substantially the final results for the transport coefficients.

\subsubsection{Method 1 for the quark relaxation time}

We start with the estimate for the quark relaxation time by means of the averaged interaction rate, related to the thermal averaged quark-quark and quark-antiquark PNJL cross sections, as advanced in Refs. [74,76-78]. We recall that the momentum-dependent relaxation time can be expressed through the on-shell interaction rate in the medium rest system, where the incoming quark has a four-momentum $P_{i}=\left(E_{i}, \mathbf{p}_{i}\right)$ :

$$
\begin{aligned}
& \tau_{i}^{-1}\left(p_{i}, T, \mu_{q}\right)=\Gamma_{i}\left(p_{i}, T, \mu_{q}\right) \\
& =\frac{1}{2 E_{i}} \sum_{j=q, \bar{q}} \frac{1}{1+\delta_{c d}} \int \frac{\mathrm{d}^{3} p_{j}}{(2 \pi)^{3} 2 E_{j}} d_{q} f_{j}^{(0)}\left(E_{j}, T, \mu_{q}\right) \\
& \times \frac{1}{16 \pi \sqrt{s}} \frac{1}{p_{c m}} \int \mathrm{d} t|\overline{\mathcal{M}}|^{2}(s, t)\left(1-f_{c}^{(0)}\right)\left(1-f_{d}^{(0)}\right) \\
& =\sum_{j=q, \bar{q}} \int \frac{\mathrm{d}^{3} p_{j}}{(2 \pi)^{3}} d_{q} f_{j}^{(0)}\left(E_{j}, T, \mu_{q}\right) v_{\mathrm{rel}} \sigma_{i j \rightarrow c d}\left(s, T, \mu_{q}\right) .
\end{aligned}
$$

The indices $\mathrm{i}$ and $\mathrm{j}$ refer to particles in the entrance channel, $\mathrm{c}$ and $\mathrm{d}$ to those in the exit channel. $f_{i}^{0}$ is the modified Fermi-Dirac distribution function taking into account the Polyakov loop (Eq. 150). $|\overline{\mathcal{M}}|^{2}$ denotes the matrix element squared averaged over the color and spin of the incoming partons, and summed over those of the final partons. The invariant energy $\sqrt{s}$ can be conveniently calculated from the four-vectors of the incoming partons. The cross section without the Pauli blocking factors is

$$
\sigma(\sqrt{s})=\int d t \frac{1}{64 \pi s p_{c m}^{2}}|\overline{\mathcal{M}}|^{2} .
$$

The relative velocity in the c.m. frame is given by

$$
v_{\text {rel }}=\frac{\sqrt{\left(p_{i} \cdot p_{j}\right)^{2}-m_{i}^{2} m_{j}^{2}}}{E_{i} E_{j}}=\frac{p_{c m} \sqrt{s}}{E_{i} E_{j}},
$$

where $p_{c m}$ is the momentum of the initial $(i, j)$ as well as of the final quarks $(c, d)$ in the c.m. frame given by

$$
p_{c m}=\frac{\sqrt{\left(s-\left(m_{i, c}-m_{j, d}\right)^{2}\right)\left(s-\left(m_{i, c}+m_{j, d}\right)^{2}\right)}}{2 \sqrt{s}} .
$$

The averaged relaxation time can be obtained from the relaxation time of Eq. (151) 


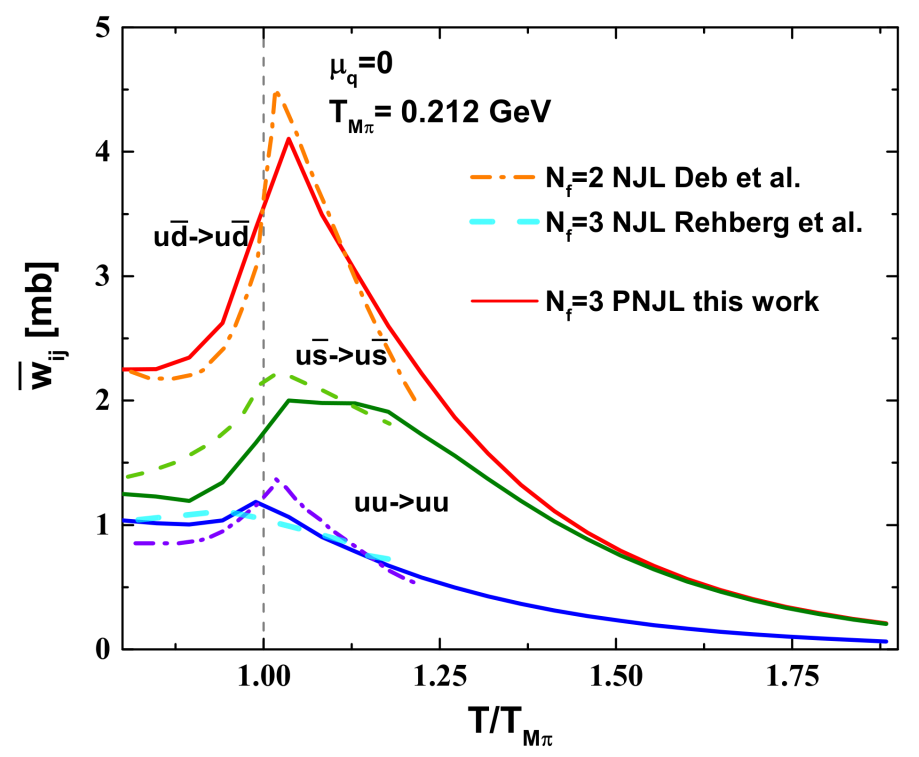

Figure 4.12: Energy averaged transition rates $\bar{w}_{i j}\left(T, \mu_{q}\right)$ for different quark-(anti-)quark scattering processes ( $u \bar{d} \rightarrow u \bar{d}$ (red and orange lines), $u \bar{s} \rightarrow u \bar{s}$ (green lines), $u u \rightarrow u u$ (blue, cyan, and violet lines)) as a function of the scaled temperature $T / T_{M \pi}$ for $\mu_{q}=0$. The solid lines corresponds to the actual results from Eq. (156). Green and cyan dashed lines correspond to the results from Ref. [186], orange and violet dash-dotted lines correspond to the estimates from Ref. [194]. The figure is taken from [5].

by averaging over $p_{i}$

$$
\tau_{i}^{-1}\left(T, \mu_{q}\right)=\frac{1}{n_{i}\left(T, \mu_{q}\right)} \int \frac{\mathrm{d}^{3} p_{i}}{(2 \pi)^{3}} d_{q} f_{i}^{(0)} \tau_{i}^{-1}\left(p_{i}, T, \mu_{q}\right)
$$

The relaxation time can be expressed via the averaged transition rate $\bar{w}_{i j}$ defined as:

$$
\bar{w}_{i j}=\frac{1}{n_{i} n_{j}} \int \frac{\mathrm{d}^{3} p_{i}}{(2 \pi)^{3}} \int \frac{\mathrm{d}^{3} p_{j}}{(2 \pi)^{3}} \times d_{q} f_{i}^{(0)} d_{q} f_{j}^{(0)} \cdot v_{\text {rel }} \sigma_{i j \rightarrow c d}\left(s, T, \mu_{q}\right) .
$$

We note, that in spite of calling $\bar{w}_{i j}$ in the literature 'averaged transition rate', it has the dimension of a cross section. Using $\bar{w}_{i j}$ defined by Eq. (156), the average quark relaxation time is given by [186]:

$$
\tau_{i}^{-1}\left(T, \mu_{q}\right)=\sum_{j=q, \bar{q}} n_{j}\left(T, \mu_{q}\right) \bar{w}_{i j}
$$

Fig. 4.12 demonstrates the actual results of the energy averaged transition rates $\bar{w}_{i j}\left(T, \mu_{q}\right)$ for three scattering processes: $u \bar{d} \rightarrow u \bar{d}$ (red and orange lines), $u \bar{s} \rightarrow u \bar{s}$ (green lines), $u u \rightarrow u u$ (blue, cyan, and violet lines) as a function of the scaled temperature $T / T_{M \pi}$ ( where $T_{M \pi}$ is the Mott temperature) for $\mu_{q}=0$ from Eq. (156) in comparison to the previous results for the NJL model from Ref. [186] (green and cyan dashed lines, $N_{f}=3$ ) and Ref. [194] (orange and violet dash-dotted lines, $N_{f}=2$ ). Our results are in a good agreement with these NJL results. A small difference arises due to different parameters of the models and different quark masses. Momentum averaged transition rates $\bar{w}_{i j}\left(T, \mu_{q}\right)$ 
4. Critical end point and 1st order phase transition of QCD matter within the PNJL model
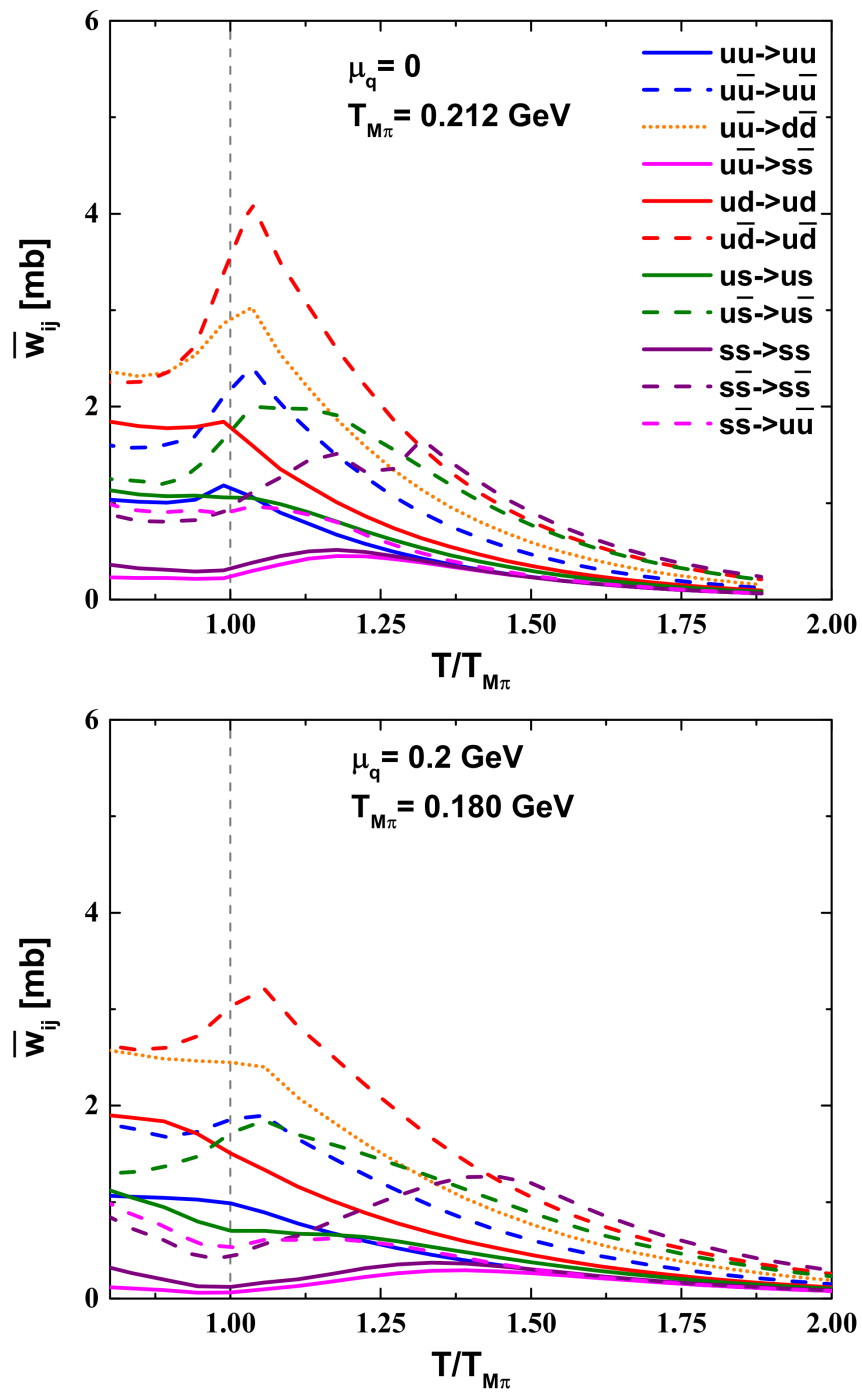

Figure 4.13: Energy averaged transition rates $\bar{w}_{i j}\left(T, \mu_{q}\right)$ for different quarkquark(antiquark) scattering processes as a function of scaled temperature $T / T_{M \pi}$ for $\mu_{q}=0$ (top) and $\mu_{q}=0.2 \mathrm{GeV}$ (bottom). The solid and dashed lines correspond to the actual results from Eq. (156) for the quark-quark and the quark-antiquark scatterings. The figure is taken from [5].

for $q q$ (solid lines) and $q \bar{q}$ (dashed lines) scattering channels are presented in Fig. 4.13 as a function of the scaled temperature $T / T_{M \pi}$ for (left) $\mu_{q}=0$ and (right) $\mu_{q}=0.2 \mathrm{GeV}$. In the vicinity of $T_{M \pi}$ the rates $\bar{w}_{i j}\left(T, \mu_{q}\right)$ have a peak, which is followed by a decrease with increasing temperature. While the values of the $q \bar{q}$ rates $\bar{w}_{q \bar{q}}\left(T, \mu_{q}\right)$ are higher than those of the $q q$ channels, the antiquark densities are smaller than the quark densities at non-zero $\mu_{q}$ (see Fig. 4.14).

\subsubsection{Method 2 for the quark relaxation time}

We continue the evaluation of quark relaxation times with an approach introduced by Zhuang [195] for the calculation of the mean-free-path and then modified by Sasaki [196] for the evaluation of the relaxation time. It is based on the 'weighted' thermal averaged quark-quark and quark-antiquark PNJL cross sections. In the dilute gas approximation 

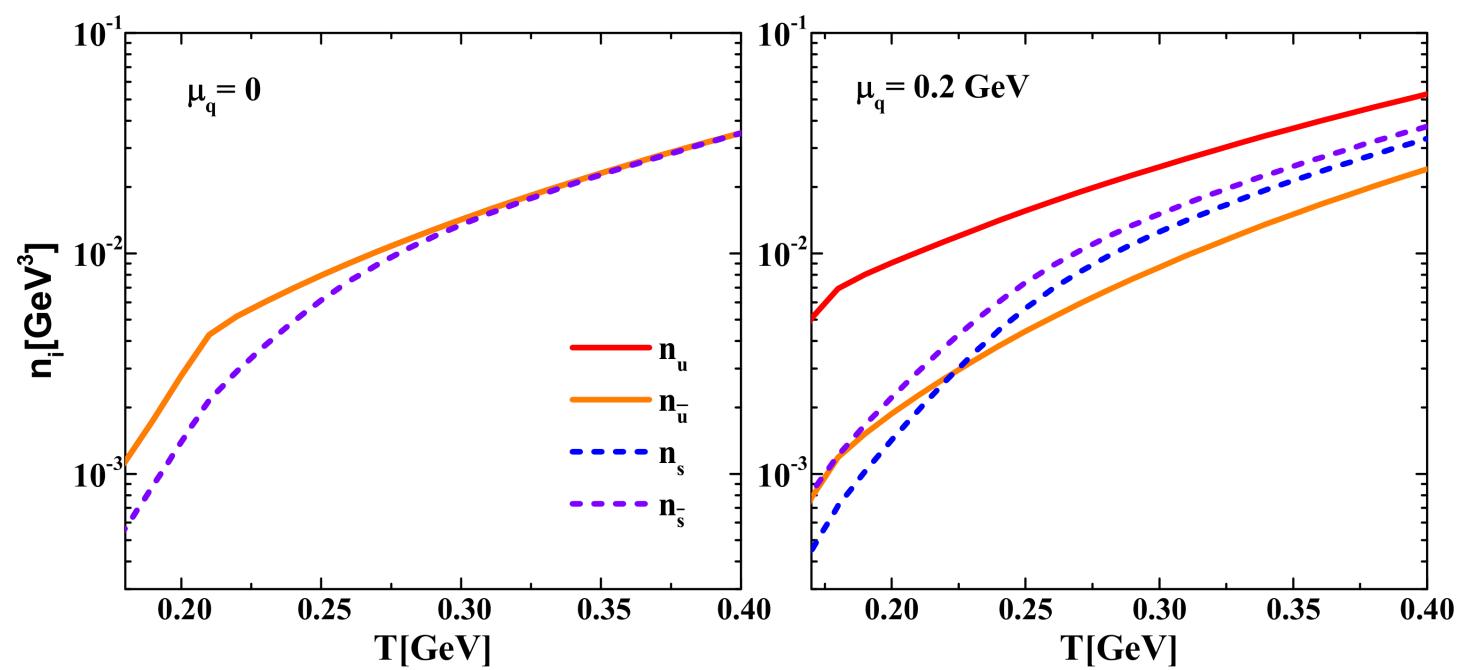

Figure 4.14: Light and strange quarks(antiquarks) densities $n_{i}\left(T, \mu_{q}\right)$ with $f_{i}^{\phi}$ - modified Fermi distributions from Eq. (150) as a function of temperature for $\mu_{q}=0$ (left) and $\mu_{q}=0.2 \mathrm{GeV}$ (right). The solid orange and red lines correspond to the light quark and antiquark densities, while the dashed blue and violet lines correspond to the strange quark and antiquark densities.

the relaxation time for the specie ${ }^{\prime} i^{\prime}$ is defined in Ref. [196] as :

$$
\tau_{i}^{-1}\left(T, \mu_{q}\right)=\sum_{j=q, \bar{q}} n_{j}\left(T, \mu_{q}\right) \bar{\sigma}_{i j}\left(T, \mu_{q}\right)
$$

In Eq. $(158) \bar{\sigma}_{i j}\left(T, \mu_{q}\right)$ are the 'weighted' thermal averaged total PNJL scattering cross sections

$$
\begin{array}{r}
\bar{\sigma}_{i j}\left(T, \mu_{q}\right)=\int_{s_{0}}^{s_{\max }} \mathrm{d} s \sigma_{i j \rightarrow c d}\left(T, \mu_{q}, s\right) P\left(T, \mu_{q}, s\right), \\
P\left(T, \mu_{q}, s\right)=C^{\prime} \int d^{3} p_{i} d^{3} p_{j} d_{q} f_{i}^{(0)}\left(E_{i}, T, \mu_{q}\right) d_{q} f_{j}^{(0)}\left(E_{j}, T, \mu_{q}\right) \\
\times \delta\left(\sqrt{s}-\left(E_{i}+E_{j}\right)\right) \delta^{3}\left(\overrightarrow{p_{i}}+\overrightarrow{p_{j}}\right) v_{r e l} .
\end{array}
$$

Here $P\left(T, \mu_{q}, s\right)$ denotes the probability of finding a quark-antiquark or quark-quark pair with a center-of-mass energy $\sqrt{s}$ and a zero total momentum. The probability $P\left(T, \mu_{q}, s\right)$ is normalised as

$$
\int_{s_{0}}^{s_{\max }} \mathrm{d} s P\left(T, \mu_{q}, s\right)=1
$$

and the relative velocity in the c.m. frame is given by Eq. (153).

For the PNJL results we use also the modified Fermi-Dirac distribution function defined by Eq. (150). Quark densities with the modified Fermi-Dirac distribution functions are shown in Fig. 4.14 as a function of the temperature for a) $\mu_{q}=0$ and b) $\mu_{q}=0.2 \mathrm{GeV}$. 
4. Critical end point and 1st order phase transition of QCD matter within the PNJL model

The relaxation time for the light quarks is defined as

$$
\begin{aligned}
\tau_{u}^{-1}\left(T, \mu_{q}\right)= & n_{u}\left(\bar{\sigma}_{u u-u u}+\bar{\sigma}_{u d-u d}\right) \\
& +n_{\bar{u}}\left(\bar{\sigma}_{u \bar{u}-u \bar{u}}+\bar{\sigma}_{u \bar{u}-d \bar{d}}+\bar{\sigma}_{u \bar{u}-s \bar{s}}+\bar{\sigma}_{u \bar{d}-u \bar{d}}\right) \\
& +n_{s} \bar{\sigma}_{u s-u s}+n_{\bar{s}} \bar{\sigma}_{u \bar{s}-u \bar{s}},
\end{aligned}
$$

and the relaxation time for strange quarks is defined as

$$
\begin{gathered}
\tau_{s}^{-1}\left(T, \mu_{q}\right)=2 n_{u} \bar{\sigma}_{u s-u s}+2 n_{\bar{u}} \bar{\sigma}_{u \bar{s}-u \bar{s}} \\
+n_{s} \bar{\sigma}_{s s-s s}+n_{\bar{s}}\left(\bar{\sigma}_{s \bar{s}-s \bar{s}}+2 \bar{\sigma}_{s \bar{s}-u \bar{u}}\right) .
\end{gathered}
$$
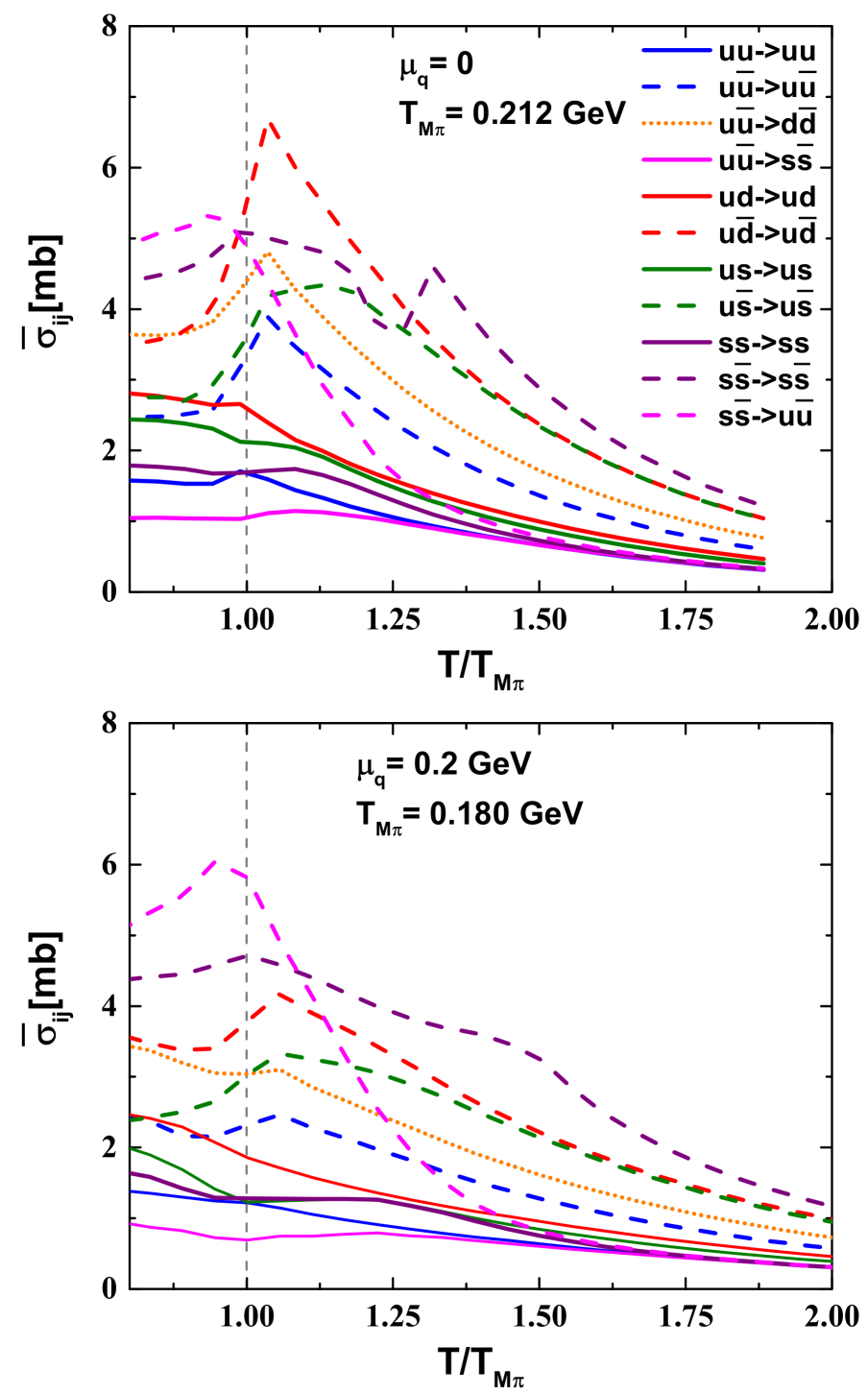

Figure 4.15: 'Weighted' thermal averaged total PNJL cross-sections $\bar{\sigma}_{i j}\left(T, \mu_{q}\right)$ from Eq. (159) as a function of the scaled temperature $T / T_{M \pi}$ for $\mu_{q}=0$ (top) and $\mu_{q}=0.2$ $\mathrm{GeV}$ (bottom). The figure is taken from [5].

The 'weighted' thermal averaged PNJL cross sections $\bar{\sigma}_{i j}\left(T, \mu_{q}\right)$ for different scattering processes are shown in Fig. 4.15 as a function of the scaled temperature $T / T_{M \pi}$ for (top) 
$\mu_{q}=0$ and (bottom) $\mu_{q}=0.2 \mathrm{GeV}$. Here $\bar{\sigma}_{i j}\left(T, \mu_{q}\right)$ shows a peak in the vicinity of the pion Mott temperature $T_{M \pi}$, which is more pronounced for the quark-antiquark $q \bar{q}$ scattering due to the peak in the cross-sections caused by the s- channel contribution. Due to this increase of the $q \bar{q}$ cross sections the 'weighted' thermal averaged cross sections $\bar{\sigma}_{i j}\left(T, \mu_{q}\right)$ for the $q \bar{q}$ channels dominates over the $q q$ channels. Approaching high temperatures (above the $\left.T_{M \pi}\right)$ the averaged cross sections $\bar{\sigma}_{i j}\left(T, \mu_{q}\right)$ decrease with temperature as expected from the behaviour of the total PNJL cross sections presented in the previous Section. The shape of the 'weighted' thermal averaged cross sections for $\mu_{q}=0$ is similar to the NJL results presented in [81], while the absolute values of the PNJL 'weighted' thermal averaged cross section $\bar{\sigma}_{i j}(T, 0)$ are larger due to different model parameters and due to the larger effective quark masses.

Using Eqs. (156),(157) and (158),(159) one can compare the underlying differences of the two methods to calculate the quark relaxation time. The first approach is simply an averaging of $v_{r e l} \cdot \sigma(\sqrt{s})$ over the momentum of the partons in the entrance channel. The second method requires in addition that the sum of the quark momenta in the entrance channel is zero and introduces an additional $\sqrt{s}$-dependence by integrating over $s$ instead of over $\sqrt{s}$. The first approach does not need any normalization whereas for the second method the normalization covers some of the parameter dependence of $P\left(s, T, \mu_{q}\right)$.
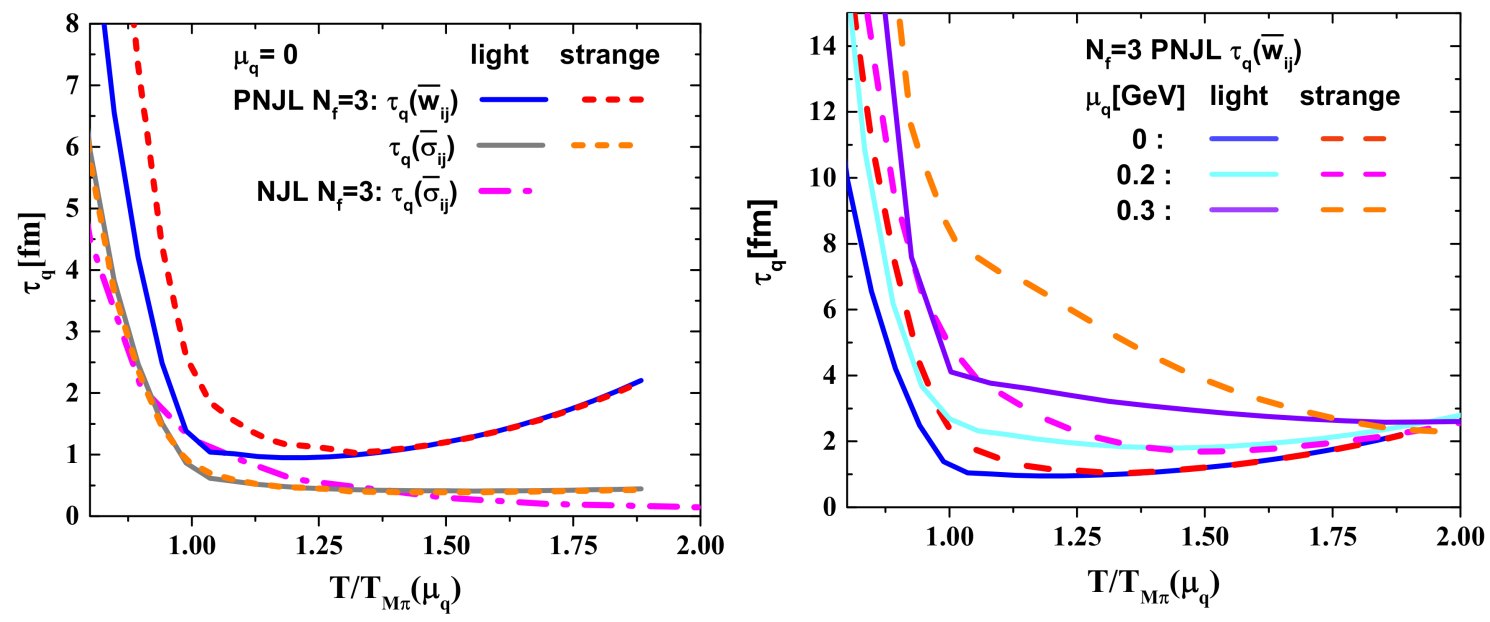

Figure 4.16: Quark relaxation times as a function of the scaled temperature $T / T_{M \pi}\left(\mu_{q}\right)$ from the PNJL model for $\mu_{q}=0$ (left) and $\mu_{q} \geq 0$ (right). The solid and the dashed lines show the results for the PNJL model using the averaged transition rates $\bar{w}_{i j}$ from Eq. (157) and the 'weighted' thermal averaged cross sections $\bar{\sigma}_{i j}$ from Eq. (158). The figure is adopted from [5].

The actual relaxation times of light and strange quarks at vanishing quark chemical potential $\mu_{q}=0$ in the PNJL model are shown in Fig. 4.16 (left) as a function of the scaled temperature $T / T_{M \pi}$. The solid gray and the dashed orange lines correspond to the actual results from Eq. (158), where the 'weighted' thermal averaged cross sections $\bar{\sigma}_{i j}$ are used. The solid blue and the dashed red lines correspond to the results from Eq. (157), where the averaged transition rates $\bar{w}_{i j}$ are used.

The difference between the two methods is most prominently seen at high temperatures. Calculations of the quark relaxation time using the averaged transition rates are 
more straight-forward since they rely on the relation between the momentum depended relaxation time and the interaction rate.

In addition we compare the quark relaxation time $\tau_{i}\left(\bar{\sigma}_{i j}\right)$ for the PNJL model with the results for the $N_{f}=3 \mathrm{NJL}$ model [81] (dashed magenta line). Our results are in a good agreement with the NJL results except for the vicinity of $T_{M \pi}$. The light quark relaxation time $\tau_{i}\left(\bar{\sigma}_{i j}\right)$ in this case is about $0.7-0.5 \mathrm{fm} / \mathrm{c}$ in the region $T_{M \pi} \leq T \leq 1.8 T_{M \pi}$.

The $\mu_{q}$-dependence of the quark relaxation time $\tau_{i}\left(\bar{w}_{i j}\right)$ is shown in Fig. 4.16 (right) for three values of $\mu_{q}: 0,0.2$ and $0.3 \mathrm{GeV}$. The solid lines correspond to the results for light quarks while the dashed lines correspond to the results for strange quarks. The quark relaxation time is increasing with the chemical potential $\mu_{q}$ in the region of $T \leq 2 T_{M \pi}$. One can see that in the vicinity of $T_{M \pi}$ the relaxation time for the strange quark is larger than that for the light quark. This difference becomes more significant for finite $\mu_{q}$ due to the difference between the effective masses of light and strange quarks.

\subsubsection{Specific shear viscosity}

Now we consider the specific shear viscosity $\eta / s$, the dimensionless ratio of the viscosity to the entropy density. The specific shear viscosity allows to compare the viscosity of liquids at various temperature scales. For this purpose we show the transport coefficients as a function of the scaled temperature $T / T_{c}$. For the PNJL calculations we use $T_{c}=T_{M \pi}$ whereas for the other approaches $T_{c}$ is the temperature of the inflection point. Here we focus on the evaluations of the transport coefficients based on the RTA.

The shear viscosity for quarks with medium dependent masses $M\left(T, \mu_{q}\right)$ can be derived using the Boltzmann equation in the RTA [76] through the relaxation time :

$$
\eta\left(T, \mu_{q}\right)=\frac{1}{15 T} \sum_{i=q, \bar{q}} \int \frac{d^{3} p}{(2 \pi)^{3}} \frac{\mathbf{p}^{4}}{E_{i}^{2}} \tau_{i}\left(T, \mu_{q}\right) \cdot d_{q} f_{i}^{\phi},
$$

where $q(\bar{q})=u, d, s(\bar{u}, \bar{d}, \bar{s}), \tau_{i}$ are the relaxation times and $f_{i}^{\phi}$ are the modified distribution functions given by Eq. (150), which contain the Polyakov loop contributions.

The specific shear viscosity $\eta / s$ for $\mu_{q}=0$ is displayed in Fig. 4.17 as a function of the scaled temperature $T / T_{c}$ in comparison to the various results from the literature. The solid and dashed red lines show the PNJL results from Eq. (177) using the two different results for the quark relaxation time: with the averaged transition rates $\bar{w}_{i j}$ from Eq. (157) and with the 'weighted' thermal averaged cross sections $\bar{\sigma}_{i j}$ from Eq. (158). The dashed gray line displays the Kovtun-Son-Starinets bound [99] $(\eta / s)_{K S S}=1 /(4 \pi)$, and the symbols show $1 \mathrm{QCD}$ data for pure $\mathrm{SU}(3)$ gauge theory, taken from Refs. [200] (squares and diamonds), [201] (circle), [85] (pentagons). The solid blue line presents the estimate for $\eta / s$ from the Bayesian analysis of the experimental heavy-ion data from Ref. [86], which has a similar temperature dependence. The result for $\eta / s\left(\bar{\sigma}_{i j}\right)$ is twice smaller than $\eta / s$ $\left(\bar{w}_{i j}\right)$ due to the different values of the quark relaxation times.

We compare the results as well with those for the $N_{f}=3$ NJL model from [81], where the relaxation time is estimated using Eq. (158) and with the DQPM prediction., where the relaxation time is calculated using the on-shell interaction rate described by Eq. (151). 


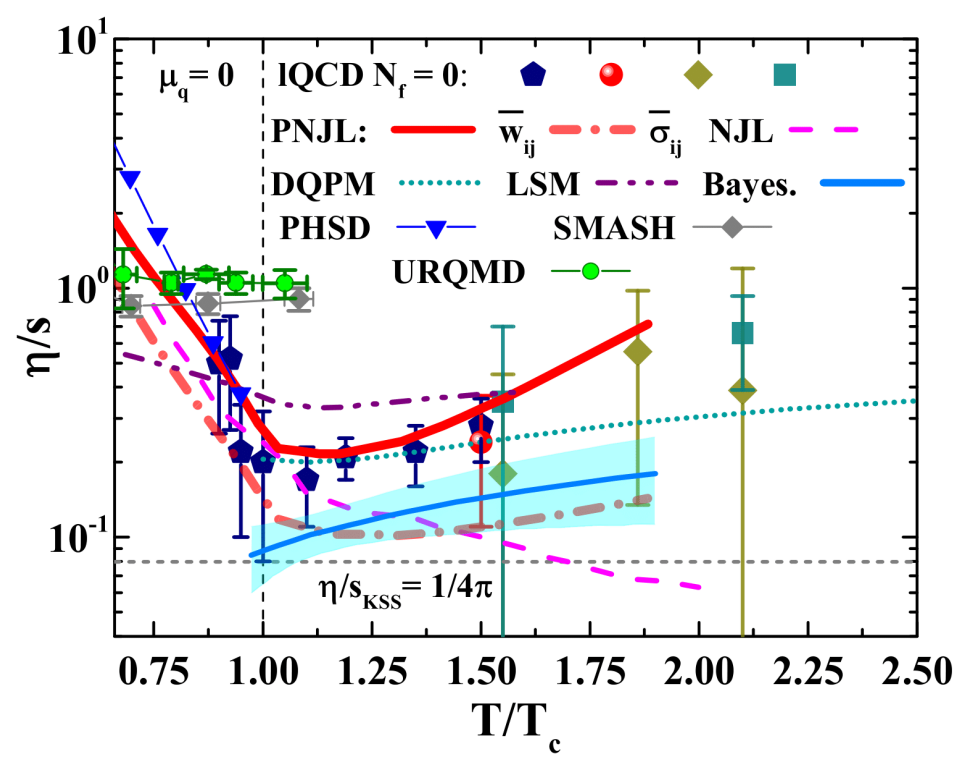

Figure 4.17: Specific shear viscosity $\eta / s$ as a function of the scaled temperature $T / T_{c}$ for $\mu_{q}=0$. The solid and the dashed red lines show the results of $\eta / s$ for the PNJL model using the averaged transition rates $\bar{w}_{i j}$ from Eq. (157) and the averaged cross sections $\bar{\sigma}_{i j}$ from Eq. (158) for the evaluation of the relaxation time. We compare to the results from various models: URQMD [197] (solid green line with circles), PHSD [84] (solid blue line with triangles), SMASH [198] (solid grey line with squares), the $N_{f}=2$ linear sigma model [199] (dashed-dotted purple line), the $N_{f}=3$ NJL model [81] (dashed magenta line), DQPM [2] (dotted green line). The dashed gray line demonstrates the Kovtun-SonStarinets bound [99] $(\eta / s)_{\mathrm{KSS}}=1 /(4 \pi)$. The symbols show lQCD data for pure $\mathrm{SU}(3)$ gauge theory taken from Refs. [200] (squares and rhombus), [201] (circle), [85] (pentagons). The solid blue line shows the results from a Bayesian analysis of experimental heavy-ion data [86]. The figure is taken from [5].

As expected, $\eta / s$ obtained within the latter method is close to the NJL estimate, and differs only at high temperature due to small differences in the cross sections, while the first method predicts a higher value for $\eta / s$, which is remarkably close to the DQPM results and to the pure $\mathrm{SU}(3)$ gauge calculations. For the hadronic phase we show the estimates from various transport models: the URQMD [197] (dotted green line), the PHSD [84] (dotted green line), and the SMASH [198] (dotted green line). The PNJL results for both methods show a similar temperature dependence in the vicinity of the chiral phase transition. Approaching the phase transition $\eta / s$ shows a dip, which is followed by an increase in the high temperature region. We consider results for non-zero chemical potential, where in the crossover region the DQPM calculations show a moderate dependence on the chemical potential (for $\mu_{u}=\mu_{s}=\mu_{B} / 3$ ), while the PNJL predictions have a more pronounced $\mu_{q}$ - dependence. As we will see later, for the whole range of the quark chemical potential both methods result in a similar temperature behavior when approaching the chiral phase transition.

The specific shear viscosity for moderate quark chemical potentials $0 \leq \mu_{q} \leq 0.3 \mathrm{GeV}$, where the phase transition is a rapid crossover, is displayed in Fig. 5.12 (left). At moderate values of $\mu_{q}$ the specific shear viscosity shows a dip after the phase transition, which is vanishing at high $\mu_{q}$ as can be seen in Fig. 5.12 (right). We compare the PNJL results with 

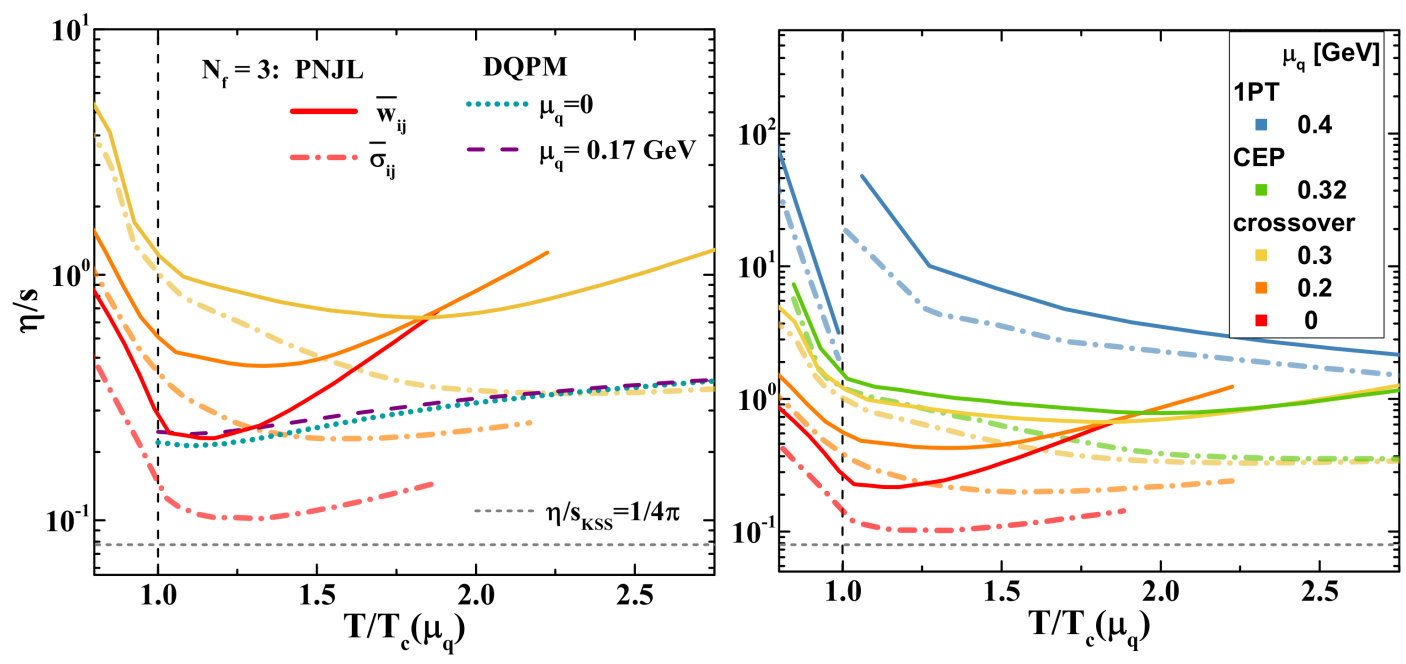

Figure 4.18: Specific shear viscosity $\eta / s$ as a function of scaled temperature $T / T_{c}$ for (left) a moderate value of the quark chemical potential $0 \leq \mu_{q} \leq 0.3$ which corresponds to a crossover phase transition and (right) whole range of the quark chemical potential $0 \leq \mu_{q} \leq 0.4 \mathrm{GeV}$. The solid (dashed) red lines show the PNJL results of $\eta / s$ for the PNJL model using the averaged transition rates $\bar{w}_{i j}(157)$ (the averaged cross sections $\bar{\sigma}_{i j}$ (158)) for the evaluation of the relaxation time. The dotted green line and dashed purple line correspond to the results from the DQPM [2] for $\mu_{q}=0$ and $\mu_{q}=0.17 \mathrm{GeV}$. The dashed gray line demonstrates the Kovtun-Son-Starinets bound [99] $(\eta / s)_{K S S}=1 /(4 \pi)$. The figure is adopted from [5].

the estimates from the DQPM for $\mu_{q}=0$ (dotted green line) and $\mu_{q}=0.17 \mathrm{GeV}$ (dashed violet line).

For large $\mu_{q}$, where the crossover transition turns into the 1st order phase transition $(1 \mathrm{PT})$, the specific shear viscosity has a discontinuity near the critical temperature. In the vicinity of the CEP, for $\mu_{q}=0.32 \mathrm{GeV}$, there is a rather smooth change of $\eta / s$, which can be seen for the crossover phase transition at $\mu_{q}=0.3 \mathrm{GeV}$. Thus, if one considers only $\mu_{q}$ values below the $\mathrm{CEP}$, the temperature dependence of the specific shear viscosity can not indicate the position of the CEP. The evolution of the specific shear viscosity with $\mu_{q}$ is in qualitative agreement with previous findings from the $N_{f}=2$ NJL model in Ref. [196]. The numerical values differ due to differences in the model parameters, distribution functions and in the NJL entropy density.

\subsubsection{Electric conductivity and the dimensionless ratio of specific shear viscosity to the scaled conductivity}

Since the QGP matter consists of charged constituents it is interesting to estimate the response of the system to an external stationary electric field, which is described by the electric conductivity $\sigma_{Q}$. We employ the RTA approach considered in Chapter 2 for the evaluation of the electric conductivity $\sigma_{Q}$ of quarks with small modifications for the PNJL model, where gluon degrees of freedom are encorporated on the level of a mean field. The 


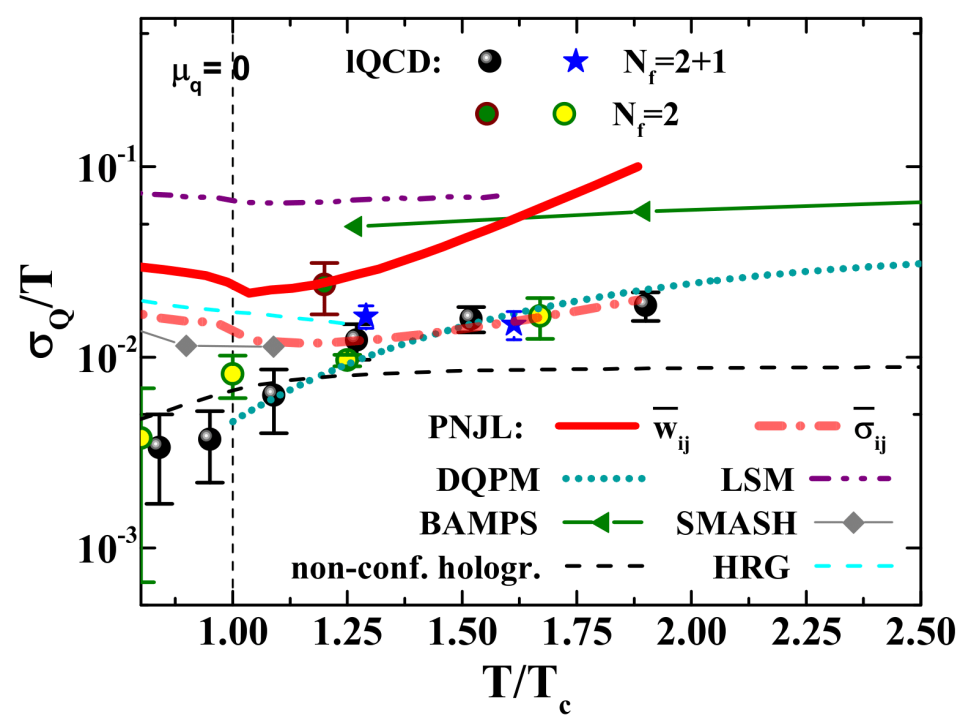

Figure 4.19: Ratio of electric conductivity to temperature $\sigma_{Q} / T$ from Eq. (164) as a function of the scaled temperature $T / T_{c}$ for $\mu_{q}=0$. The solid (dashed) red lines show the PNJL results of $\sigma_{Q} / T$ from the PNJL model using the averaged transition rates $\bar{w}_{i j}$ from Eq. (157) (the 'weighted' thermal averaged cross sections $\bar{\sigma}_{i j}$ from Eq. (158)) for the evaluation of the relaxation time. The symbols display lQCD data for $N_{f}=2$ taken from Refs. [94,94] (red circles with brown borders), (yellow circles with green borders) and for $N_{f}=2+1$ taken from Refs. [96] (spheres) and from Ref. [111] (blue stars). We compare to predictions from the various models: the kinetic partonic cascade model BAMPS [112] (the dark-green solid line with triangles), the non-conformal holographic EMD model [49] (dashed black line), the DQPM [2] (dotted green line), and below $T_{c}=0.158 \mathrm{GeV}$ we show evaluations from hadronic models: the HRG model within the Chapman-Enskog expansion of the BE [52,104] (dashed cyan line), the $N_{f}=2$ linear sigma model [199] (dashed doted purple line), SMASH [105,114] (solid grey line with squares). The figure is taken from [5].

electric conductivity $\sigma_{Q}$ with the effective masses $M\left(T, \mu_{q}\right)$ in the RTA reads,

$$
\sigma_{Q}\left(T, \mu_{q}\right)=\frac{e^{2}}{3 T} \sum_{i=q, \bar{q}} q_{i}^{2} \int \frac{d^{3} p}{(2 \pi)^{3}} \frac{\mathbf{p}^{2}}{E_{i}^{2}} \cdot \tau_{i}\left(T, \mu_{q}\right) d_{q} f_{i}^{\phi},
$$

where $d_{q}=2 N_{c}=6$ is the degeneracy factor for spin and color in case of quarks and antiquarks, $\tau_{i}$ are their relaxation times, while $f_{i}^{\phi}$ denote the modified distribution functions for quark and anti-quarks defined by Eq (150). In these formula we deal with quarks and anti-quarks of $N_{f}=3$ flavours. Each quark has a contribution proportional to its charge squared. While viscosities have in general a gluonic contribution, the electric conductivity contains only a quark and anti-quark contribution.

The PNJL results for the dimensionless ratio of the electric conductivity to temperature $\sigma_{Q} / T$ for $\mu_{q}=0$ are presented in Fig. 4.19 for both methods of the calculation of the quark relaxation time by solid and dashed red lines. We compare the PNJL results to the various estimates from the literature: $1 Q C D$ data for $N_{f}=2$ taken from Refs. [94,95] (red circles with brown borders), (yellow circles with green borders) and for $N_{f}=2+1$ taken from Refs. [96, 116] (spheres) and from Ref. [111] (blue stars); kinetic partonic cascade model BAMPS [112] (the dark-green solid line with triangles), non-conformal holographic 

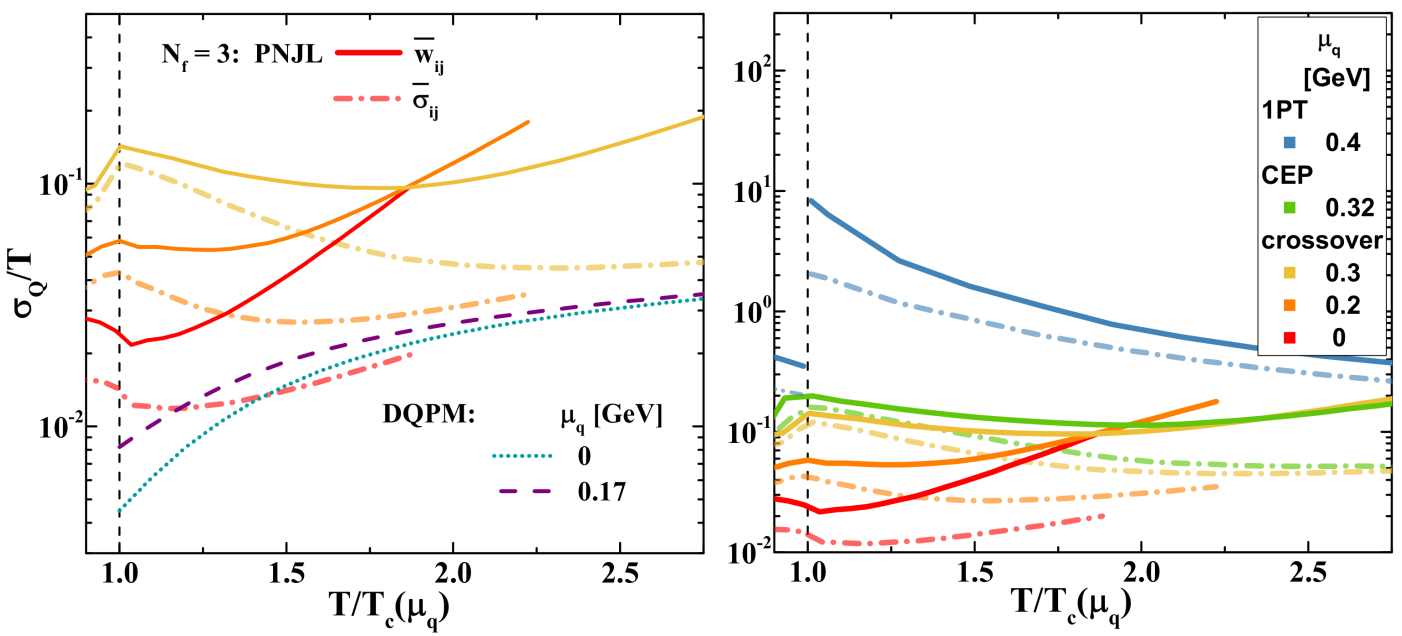

Figure 4.20: Ratio of the electric conductivity to the temperature $\sigma_{0} / T$ as a function of the scaled temperature $T / T_{c}$ for $\mu_{q}=0$ (left) and $\mu_{q} \geq 0$ (right). The solid (dashed) red lines show results of $\sigma_{Q} / T$ from the PNJL model using the averaged transition rates $\bar{w}_{i j}$ from Eq. (157) (the 'weighted' thermal averaged cross sections $\bar{\sigma}_{i j}$ from Eq. (158)) for the evaluation of the relaxation time. The dotted green line and dashed purple line correspond to the results from the DQPM [2] for $\mu_{q}=0$ and $\mu_{q}=0.17 \mathrm{GeV}$. The figure is adopted from [5].

EMD model [49] (dashed black line), DQPM [2] (dotted green line), and below $T_{c}=$ $0.158 \mathrm{GeV}$ we show evaluations from hadronic models: HRG model within the ChapmanEnskog expansion of the BE [52,104] (dashed cyan line), $N_{f}=2$ linear sigma model [199] (dashed doted purple line), and SMASH [105,114] (solid grey line with squares). The PNJL results for both methods of quark relaxation time predict a similar increase with temperature, which is again due to the increase of the quark densities with temperature. The temperature dependence is in agreement with the predictions from the DQPM [2] despite the differences in the effective masses.

The chemical potential dependence is shown in Fig. 4.20 (left) for moderate values of $\mu_{q}$ and (right) for the whole range of $\mu_{q}$. As in case of the specific shear viscosity also the electric conductivity has a discontinuity at the 1st order phase transition (and hence for $\left.\mu_{q}>0.32\right)$. At lower chemical potentials, where the transition is a crossover, $\sigma_{Q} / T$ is a continuous function of the temperature. Starting from $\mu_{q}=0$, with increasing $\mu_{q}$ $\sigma_{Q} / T$ has first a dip approaching the phase transition temperature, which, for a moderate value of $\mu_{q}=0.2-0.3 \mathrm{GeV}$, turns into a hump before at $\mu_{q}=0.4 \mathrm{GeV}$, where the phase transition is of 1 st order, it shows a discontinuity. For low values of $\mu_{q}$ and above the chiral phase transition, $T \leq 2 T_{C}, \sigma_{Q} / T$ is raising with $\mu_{q}$, which is in agreement with the DQPM estimates [2], and predictions from the non-conformal holographic model in Ref. [117].

Furthermore, it is interesting to compare the momentum diffusion, described by the specific shear viscosity $\eta / s$, and the charge diffusion, described by the scaled electric conductivity $\sigma_{Q} / T=\kappa_{Q} / T^{2}$ ( $\kappa_{Q}$ is the charge diffusion coefficient) by calculating the ratio $\frac{\eta / s}{\sigma_{Q} / T}$. This ratio is less dependent on the approximations made for the evaluation of the quark cross sections or quark relaxation times. Figure 4.21 shows the ratio $\frac{\eta / s}{\sigma_{Q} / T}$ as a 


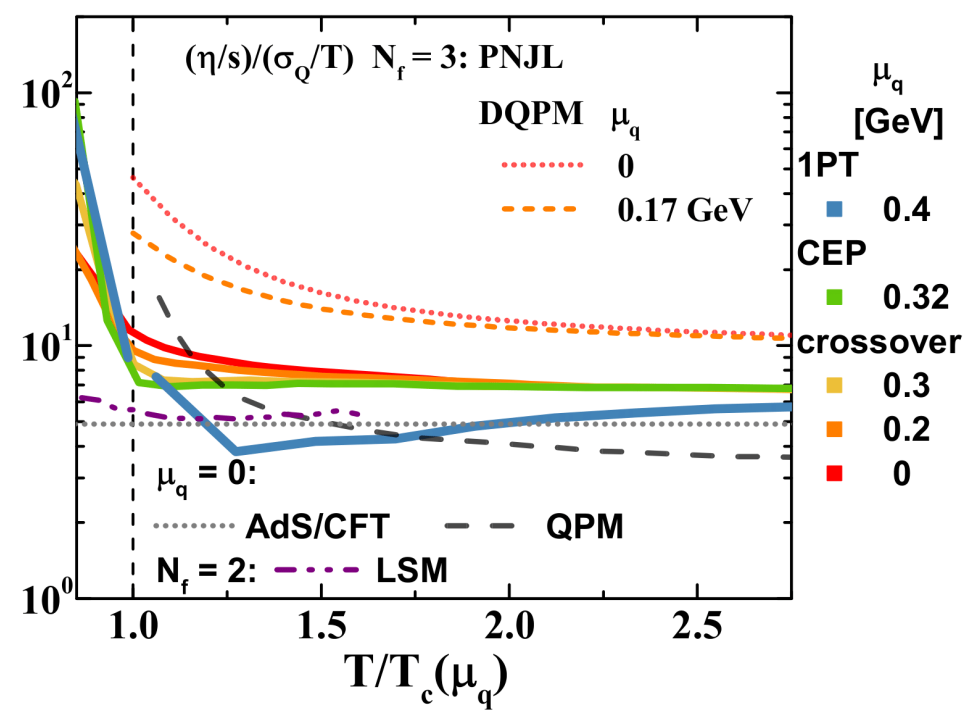

Figure 4.21: Ratio of specific shear viscosity $\eta / s$ to the scaled electric conductivity $\sigma_{Q} / T$ as a function of scaled temperature $T / T_{c}$ for $\mu_{q} \geq 0$. For $\mu_{q}=0$ we show the estimations from various models: the QP model [98] (dashed grey line), the DQPM [2] (dotted red line), the AdS/CFT [99, 202] (dotted grey line), the $N_{f}=2$ linear sigma model [199] (dashed doted purple line). The figure is taken from [5].

function of the scaled temperature $T / T_{c}$ for a range of quark chemical potentials $\mu_{q} \geq 0$.

At vanishing quark chemical potential $\mu_{q}=0$ we compare our results to the predictions from the AdS/CFT (grey dotted line) [99,202], evaluations from the $N_{f}=2$ linear sigma model [199] (dashed-dotted purple line), the DQPM predictions [2] (green dash-dotted line) and estimates from the on-shell quasiparticle (QP) model (blue dashed line) [98], where the effective coupling constant is given by a one-loop pQCD ansatz and is higher than the effective coupling constant in the DQPM, which results in higher effective masses of partons compared to the DQPM masses. Note that the QPM predicts a higher value of the electric conductivity compared to the DQPM and lQCD results. For the PNJL calculations we see for $\mu_{q}$ values, where the phase transition is assumed to be a crossover, below $T_{C}$ a strong decrease of this ratio with temperature, whereas above $T_{C}$ the ratio flattens out. For $\mu_{q}$ values, where a first order phase transition is observed, we see also for this ratio a discontinuity which we already observed for the viscosity and the electric conductivity.

It has to be mentioned that the 3-dimensional mean field models are conceptually not accurate near the critical point and the first order phase transition [203]. They are built on the anzatz that the fluctuations are small compared to the average value, while approaching the critical point the correlation length becomes large and diverges at the critical point. The feature of the PNJL and NJL models regarding the static critical exponents, the size of the critical region and the influence of the Polyakov loop have already been studied in Refs. [204-206].

In the vicinity of the critical region one has to consider additional critical contributions governed by the dynamics of the fluctuations associated with the CEP. The dynamical universality class of the QCD critical point is argued to be that of the $\mathrm{H}$ model $[102,207]$ according to the classification of dynamical critical phenomena by Ho- 

PNJL model

henberg and Halperin [208]. Whereas in the vicinity of the CEP the shear viscosity has a mild divergence in the critical region, the bulk viscosity has a more pronounced divergence [102, 208, 209]: $\eta \sim \xi_{T}^{Z_{\eta}}\left(Z_{\eta} \approx 1 / 19\right), \zeta \sim \xi_{T}^{Z_{\zeta}}\left(Z_{\zeta} \approx 3\right)$, and the electric conductivity diverges as $\sigma_{Q} \sim \frac{1}{\xi_{T}}$, where $\xi_{T} \sim\left(T-T_{C}\right)^{\nu}$ is the thermal correlation length, with $\nu$ being the static critical exponent. The specific bulk and shear viscosities have been considered near the CEP and the 1st-order phase transition for the $N_{f}=2 \mathrm{NJL}$ model in the previous study [196]. Therefore the presented results can qualitatively describe $\eta / s$ and $\sigma / T$ above $T_{C}$, and a further development of the critical contribution to the transport coefficients in the critical region is needed. Recently a generic extension of hydrodynamics by a parametrically slow mode or modes ("Hydro+") and a description of fluctuations out-of equilibrium have been considered in Ref. [210]. 


\subsection{Conclusions to Section 4}

We have calculated the specific shear viscosity $\eta / s$ and the ratio of electric conductivity to temperature $\sigma_{Q} / T$ of the QGP matter in the extended PNJL model for a wide range of quark chemical potentials using the framework of the Boltzmann equation in the relaxation time approximation.

We showed that both, the specific shear viscosity $\eta / s$ and the ratio of the electric conductivity to the temperature, $\sigma_{Q} / T$, depend strongly on the chemical potential.

Furthermore, we demonstrated the dependence of the transport coefficients on the quark relaxation times, which were estimated within two methods: either by using the averaged transition rates $\bar{w}_{i j}$ or by the 'weighted' thermal averaged cross sections $\bar{\sigma}_{i j}$. The evaluation made within the first method is considered to be more realistic as it stems from the derivation of the relaxation time through the interaction rate. In the vicinity of the chiral phase transition both methods result in a similar temperature dependence of the considered transport coefficients, which for a vanishing quark chemical potential are in agreement with various results from the literature. They include the results for the specific shear viscosity $\eta / s$ and the ratio of the electric conductivity and the temperature, $\sigma_{Q} / T$, obtained with the $N_{f}=3 \mathrm{NJL}$ model [81], lattice QCD predictions, the $N_{f}=2$ linear sigma model, predictions from the transport models such as URQMD, BAMPS, SMASH, PHSD and estimates from the DQPM. In the vicinity of the pseudo-critical temperature our results are remarkably close to that of $1 Q C D$ calculations and to the results from the DQPM.

- The key result of this Chapter is the quark chemical potential $\mu_{q}$-dependence of the considered transport coefficients. It has been demonstrated that for moderate values of $\mu_{q}\left(\mu_{q} \leq 0.3 \mathrm{GeV}\right)$, where the phase transition is a rapid crossover, transport coefficients show a smooth temperature dependence while approaching the (pseudo)critical temperature from the high temperature region.

- At large values of $\mu_{q}$ the presence of a first order phase transition changes the temperature dependence of the transport coefficients drastically and a discontinuity can be seen when approaching the critical temperature.

- We found that outside the critical region the influence of the CEP on the evaluated transport coefficients is rather small in comparison to the modification due to a 1st order phase transition. For the specific shear viscosity a similar behaviour near the chiral phase transition has been obtained in the $N_{f}=2$ NJL model in Ref. [196].

- We have considered, furthermore, the dimensionless ratio of specific shear viscosity to the scaled electric conductivity. It shows as well a discontinuity at $T_{c}$ if the chiral transition is of 1 st order but is otherwise almost constant for $T>2 T_{c}$. 


\section{Transport properties of the QGP in the dynamical quasiparticle model with a CEP}

This Chapter is devoted to the exploration of the transport properties of the QGP matter in the high $\mu_{B}$ region, where a CEP is incorporated. To this aim a phenomenological extension of the DQPM, described in detail in Chapter 2, to large baryon chemical potentials $\mu_{B}$ including a critical end-point and a 1st-order phase transition is considered. In the DQPM the determination of complex self-energies for the partonic degrees of freedom at zero and finite $\mu_{B}$ has been performed by adjusting the entropy density to the lQCD data. The temperature-dependent effective coupling (squared) $g^{2}\left(T / T_{c}\right)$, as well as the effective masses and widths of the partons are based on this adjustment.

The novel extended dynamical quasiparticle model, named "DQPM-CP", enables to describe simultaneously the macroscopic properties such as thermodynamic observables as well as microscopic properties of the partonic phase, such as scattering cross-sections, effective masses and widths and transport coefficients of quarks and gluons in a wide range of temperatures $T>T_{c}$, and baryon chemical potentials $\mu_{B}$. The model parameters are fixed to reproduce the EoS of lattice QCD calculations in the crossover region of finite $T, \mu_{B}$, whereas at high $\mu_{B}$ it approaches the region of the CEP and first-order phase transition, while it reproduces the asymptotic behaviour from pQCD calculations for $\mu_{B}>>T, \mu_{B} \sim 1-2 \mathrm{GeV}$.

To include the scaling behaviour of the thermodynamic observables and microscopic properties we consider a scaling ansatz for the strong coupling constant near the critical endpoint $\mathrm{CEP}$, chosen at $\left(T^{C E P}, \mu_{B}^{C E P}\right)=(0.100,0.960) \mathrm{GeV}$. We show the equation-of-state as well as the speed of sound for $T>T_{c}$ for a wide range of $\mu_{B}$, which should be of interest for hydrodynamical simulations.

Moreover, one of the advantages of the quasiparticle models is a simple implementation in transport models. For instance the on-shell quasiparticle model [211] has been successfully used in the Catania transport approach [212] for the evolution of the partonic medium by means of the relativistic Boltzmann equation, and - as mentioned above - the DQPM has been implemented in the PHSD transport approach, which describes the whole evolution of the QCD medium as well as the phase transition between the hadronic and partonic phase and vice versa. Furthermore, the DQPM allows to employ covariant transition rates for the phase transition, which obeys strict 4-momenta conservation during the transition in the PHSD.

For the application of the partonic EoS in simulations of the medium produced in the 
HICs, it is essential to consider strangeness neutrality. To this aim we consider two settings for the strange quark chemical potentials (I) $\mu_{q}=\mu_{u}=\mu_{s}=\mu_{B} / 3$ and (II) $\mu_{s}=0, \mu_{u}=\mu_{d}=\mu_{B} / 3$. Furthermore, we present the isentropic trajectories of the QGP matter for these two cases.

Transport coefficients of the partonic phase are calculated within the RTA method, as described in Chapter 2, for a wide range of baryon chemical potentials. The influence of the $\mathrm{CEP}$ on the transport coefficients is considered as well. We compare the thermodynamic observables and transport coefficients to the results from the PNJL model considered in the previous Chapter. Despite that the phase diagram of the DQPM-CP is close to the PNJL calculations the transport coefficients of both approaches differ. This elucidates that the knowledge of the phase diagram alone is not sufficient to describe the dynamical evolution of strongly interacting matter. 


\subsection{Universal scaling approaching a critical end-point}

The main concept of the theory of phase transitions and critical phenomena is the universality principle, i.e. independence of thermodynamic characteristics of different systems at phase transitions from differences in the values of small-scale parameters which allows to divide all systems into a small number of universality classes, depending on a number of the spatial dimensions of the system and the symmetry of its order parameter. According to the conventional classification of phase transitions proposed by Ehrenfest [213], a first-order phase transition characterised by the discontinuity of the first-order derivatives of the thermodynamic potential, while in the case the second-order phase transition only second-order derivatives have a discontinuity. Long range space-correlations manifest the singularity in the thermodynamic observables, while long-range time-correlations to non-equilibrium phenomena, which can be described by a singular behavior of transport coefficients.

Close to the second-order phase transition the characteristic sizes disappear from the physical description and power-law distributions come forth and characterize the physical quantities. The exponents of these power-laws, i.e. the critical exponents [209] become the relevant observables. To generalize description for the various systems with different critical temperatures a scaled temperature is considered:

$$
t=\frac{T}{T_{c}}
$$

where for the systems considered below at non-zero baryon density or equivalently, finite baryon chemical potential $T_{c}$ varies with $\mu_{B}$. The quite remarkable amount of experimental data have shown that second-order phase transitions of apparently quite different systems can be characterized by the same set of critical exponents [209]. This phenomenon is called universality. For instance, critical exponents at the critical point of the liquidgas phase transition have been found to be independent of the fluid chemical composition. Furthermore, they are exactly the same as the critical exponents of the ferromagnetic phase transition in uniaxial magnets, which is the same as that of the 3-dimensional Ising model. Such systems are then referred to as being in the same universality class. Furthermore, the renormalization group theory of second-order phase transitions postulates that the thermodynamic properties of a system near a critical point depend only on a small number of features such as the dimensionality of the system and the symmetry of the interactions, but are insensitive to the underlying microscopic properties.

\subsection{Extension of the quasiparticle DQPM-CP effective cou- pling constant for the inclusion of a CEP}

Now we proceed with the extension of the DQPM to the region of large $\mu_{B}$, where a possible critical end-point is located. In order to extent the quasiparticle model to the large $\mu_{B}$ region and to describe the critical behavior near the CEP we depart from the 'scaling hypothesis' used for the moderate baryon chemical potentials in the crossover region, and 


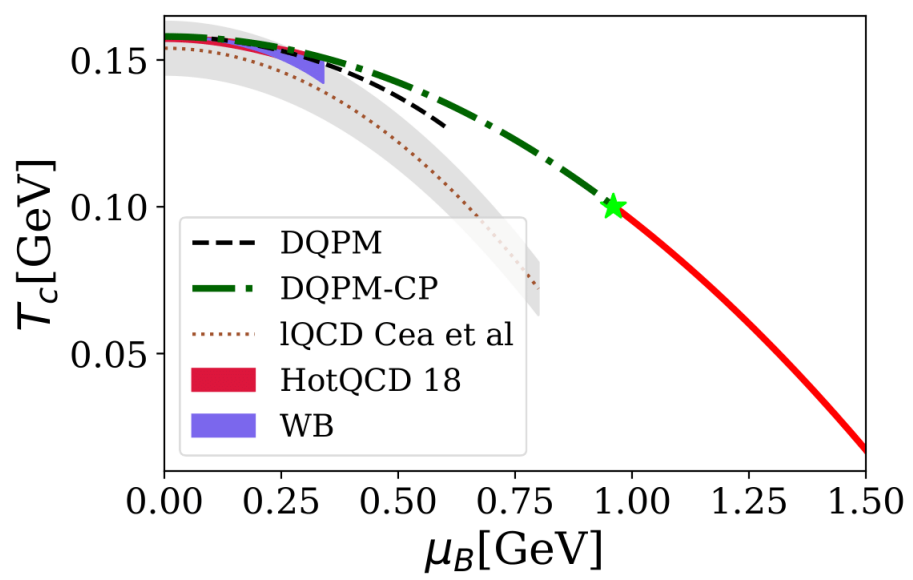

Figure 5.1: Critical line of the DQPM-CP (black dashed-dotted and red solid lines) and of the DQPM (green dashed line) in the $T-\mu_{B}$-plane of the QCD phase diagram. Lines with colored areas represent lQCD estimates of $T_{c}\left(\mu_{B}\right)$ for QCD with $N_{f}=2+1$ : grey area - from Ref. [214], red area - from Ref. [192], blue area - from Ref. [21].

introduce a simple parametrization of the coupling constant as a function of the scaled temperature and the baryon chemical potential. To simplify an extension of the effective coupling for finite $\mu_{B}$ we parametrize $\alpha_{s}^{D Q P M-C P}$ as a function of the dimensionless scaled temperature $t=T / T_{c}$. We determine first the parameters at vanishing quark or baryon chemical potential by fitting the extracted values of $g_{D Q P M}^{2}\left(T, \mu_{B}=0\right)$ from Eq. (28) as a function $f\left(T / T_{c}, \mu_{B}=0\right)\left(T_{c}=0.158 \mathrm{GeV}\right)$ with help of the nonlinear least-squares (NLLS) Marquardt-Levenberg algorithm.

Later we use critical lines of $T_{c}$ for each value of the baryon chemical potential. In the DQPM, the critical line is an input parameter, which can be chosen from recent LQCD calculations or from the estimates of other theoretical approaches. Here we define the critical line as follows,

$$
T_{c}=T_{c}(0)\left[1-\kappa_{P N J L}\left(\frac{\mu_{B}}{T_{c}(0)}\right)^{2}\right],
$$

where $T_{C}(0)=0.158 \mathrm{GeV}$ is chosen in accordance with the results from lQCD [21,192], while $\kappa_{P N J L}=0.00989$ corresponds to the estimate from the PNJL model [175].

Figure 5.1 shows a comparison of the critical lines of the original DQPM (green dashed line), of the extended DQPM-CP and the predictions from IQCD calculations. The DQPMCP phase boundary, given by Eq. (166), is shown as a black dashed-dotted line in the crossover region, i.e. for moderate baryon chemical potentials. Furthermore, we consider a critical endpoint, which is located at $\left(T^{C E P}, \mu_{B}^{C E P}\right)=(0.100,0.960) \mathrm{GeV}$ according to the critical $\mu_{B}^{C E P}=0.960 \mathrm{GeV}$ from the PNJL model [175]. The first-order phase transition is shown by a solid red line. The DQPM phase boundary for moderate baryon chemical potentials, $\mu_{B} \leq 0.6 \mathrm{GeV}$, given by Eq. (30), is displayed by a dashed green line. The colored areas illustrate the predictions from 1 QCD calculations for QCD with $N_{f}=2+1$ : grey area - from Ref. [214], red area - from Ref. [192], blue area - from Ref. [21]. Note that the critical line from Ref. [214], has a higher curvature and therefore lies above the 
predictions from the HotQCD and BMW collaborations. Moreover, the curvature parameter obtained within the 1QCD approach, can differ for the different observables that are used to define the parameter [215].

As one can see the chosen critical line agrees well with the $1 Q C D$ predictions in the region of moderate baryon chemical potentials.

We extend the effective coupling, assuming that the coupling constant has a similar dependence as a function of the scaled temperature for all $\mu_{B} \geq 0$. Furthermore we introduce the additional factor $\sigma\left(\mu_{B}\right)\left(\sigma\left(\mu_{B}=0\right)=1\right)$ for a decrease of the coupling constant with $\mu_{B}$. Therefore, the DQPM-CP coupling constant can be parametrized as a function of the scaled temperature $\left(t=T / T_{c}\left(\mu_{B}\right)\left(T_{c}\right.\right.$ varies with $\mu_{B}$ according to the DQPM critical line) and $\mu_{B}$ :

$$
\alpha_{S}^{\text {cross }}=a_{0}+\frac{a_{2}}{t^{2}}-\frac{a_{3}}{t^{3}}+\frac{a_{4}}{t^{4}}+\frac{a_{6} \cdot \sigma\left(\mu_{B}\right)}{t^{6}} .
$$

Here the coefficients $a_{i}$ are fixed at $\mu_{B}=0$ (where $\sigma\left(\mu_{B}=0\right)=1$ ) by fitting the DQPM coupling constant $g^{2}\left(T, \mu_{\mathrm{B}}=0\right)$ obtained from Eq. (28) (see comparison of the basic thermodynamic observables from DQPM-CP and lQCD predictions in Fig. 5.2): $a_{0}=0.25$, $a_{2}=1.77, a_{3}=2.17, a_{4}=2.13, a_{6}=0.85$. Moreover, the QGP matter is expected to

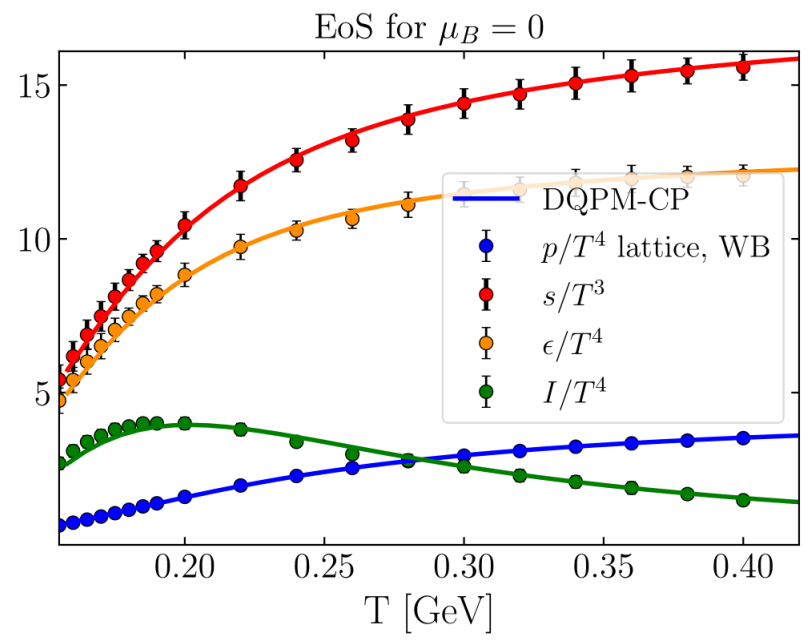

Figure 5.2: The scaled pressure $P(T) / T^{4}$ (blue line), entropy density $s(T) / T^{3}$ (red line), scaled energy density $\epsilon(T) / T^{4}$ (orange line), and interaction measure $I(T) / T^{4}$ (green line), from the DQPM-CP in comparison to the lQCD results from Refs. [22,60] (circles) for zero baryon chemical potential.

approach the non-interacting Stefan-Boltzmann limit at large $\mu_{B}$ on the order of a few $\mathrm{GeV}$ and small temperatures $\mu_{B} \gg T$ (see recent HTL results on the pressure in [190]). Therefore, it is reasonable to assume that the coupling constant also decreases at $T_{c}$ with increasing $\mu_{B}$, whereas at higher $T \gg T_{c}$ and finite $\mu_{B}$, the effective coupling is not sensitive to $\mu_{B}$.

The simplest way to introduce a decrease in the coupling constant is to consider the 


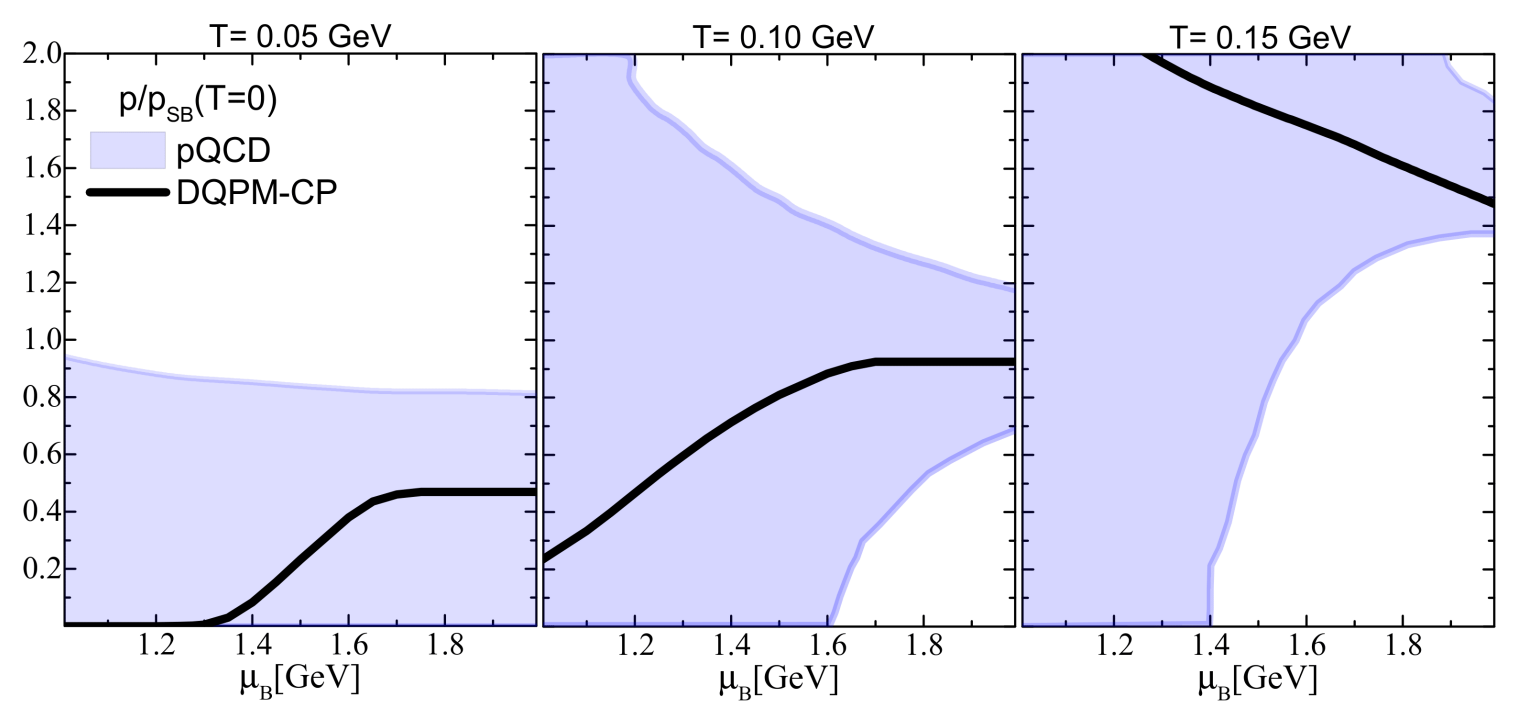

Figure 5.3: The scaled pressure $p / p_{S B}$ (black line) from the DQPM-CP in comparison to the pQCD results from Ref. [190] (blue area) as a function of baryon chemical potential for fixed temperatures $T=0.05,0.10,0.150 \mathrm{GeV}$. Scenario: $\mu_{q}=\mu_{u}=\mu_{s}=\mu_{B} / 3$.

additional factor, affecting the region near the phase transition:

$$
\sigma\left(\mu_{B}\right)=1-\sigma_{2} \mu_{B}^{2}-\sigma_{4} \mu_{B}^{4}
$$

where $\sigma_{2}=0.45 \mathrm{GeV}^{-2}$ and $\sigma_{4}=0.15 \mathrm{GeV}^{-4}$. Here the parameters $\sigma_{2}$ and $\sigma_{4}$ are fixed by fitting the quasiparticle entropy from Eq. (36) to the $1 \mathrm{QCD}$ data of the entropy density from the BMW collaboration [22,60] for moderate chemical potentials $\mu_{B}=0.1,0.2,0.3,0.4$ $\mathrm{GeV}$ within the given temperature range $T_{c}\left(\mu_{B}\right)<T<T_{\max }$, where $T_{c}\left(\mu_{B}\right)$ denotes the chosen line for the model critical temperature and $T_{\max }=0.4 \mathrm{GeV}$.

We note that the adjustment the effective coupling constant is made in order to interpolate results for thermodynamic observables and microscopic quantities from the region of vanishing baryon chemical potential to the high baryon chemical potential region, where high and moderate temperatures above the phase transition line $T>T_{c}\left(\mu_{B}\right)$ are considered.

The aim of the model is to describe the qualitative behavior of the thermodynamic observables in the regions of high/moderate baryon density for $T>T_{c}\left(\mu_{B}\right)$, while in the region of moderate baryon chemical potential - to reproduce the IQCD EoS. To verify the considered $\mu_{B}$-dependence of the effective coupling we consider the region of the high chemical potentials $\mu_{B}>>T$, where the results from pQCD calculations are available. We compare the ratio of the resulting pressure to the non-interacting Stefan-Boltzmann limit for high chemical potential $p / p_{S B}(T=0)\left(p_{S B}(T=0)=\frac{\mu_{B}^{4}}{108 \pi^{2}}\right)$ from DQPM-CP for high $\mu_{B}$ with the pQCD calculations from Ref. [190] in Fig. 5.3. One can see that the depicted ranges of the $\mathrm{PQCD}$ results are quite broad and the resulting pressure from the DQPM-CP is within the $\mathrm{pQCD}$ predictions.

To accumulate 'critical' behaviour near the CEP, where the phase transition is of secondorder, we use an additional 'critical' term for the coupling constant. The goal of this term is to describe the critical behaviour at the second-order phase transition for the microscopic 


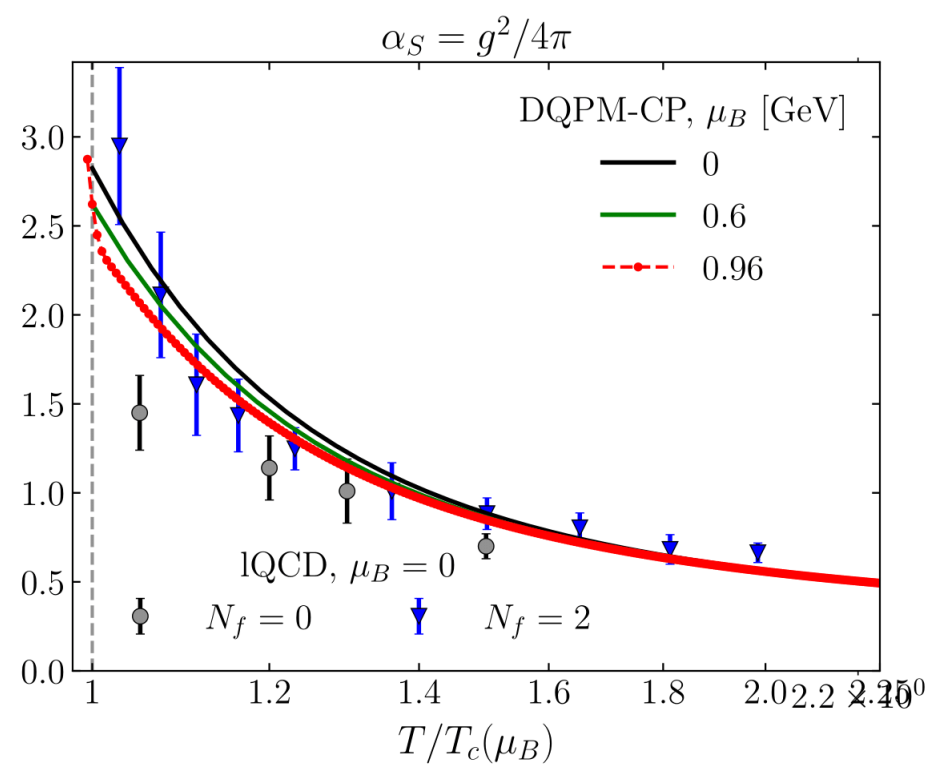

Figure 5.4: The running coupling $\alpha_{S}^{D Q P M-C P}$ from Eq. (170) as a function of the scaled temperature $T / T_{c}$ at fixed $\mu_{B}$ : for $\mu_{\mathrm{B}}=0, T_{c}=0.158 \mathrm{GeV}$ (red solid line) corresponds to the crossover phase transition and $\mu_{\mathrm{B}}=0.96, T_{c}=0.10 \mathrm{GeV}$ (green points) corresponds to the CEP. The lattice results for quenched QCD, $N_{f}=0$, (black circles) are taken from Ref. [58] and for $N_{f}=2$ (blue triangles) are taken from Ref. [59].

and thermodynamic quantities.

As shown in Section 2 in the quasiparticle picture the effective coupling constant enters the entropy density, moreover the specific heat, which - as mentioned above - diverges at the CEP, is related to the derivative of the coupling constant $C_{V} \sim \frac{\partial g}{\partial T}$. Therefore it is expected that in the vicinity of the CEP, where the specific heat diverges, the effective coupling constant should attain the increase approaching the CEP form high T. One can interpret the increase of the effective coupling constant, when approaching the CEP, as an increase of the interaction range governed by the correlation length $\xi_{T} \sim\left(T-T_{c}\right)^{\nu}$.

To adopt the increase of the coupling constant when approaching the CEP, we consider a parametrization of the 'critical' coupling constant, which is fixed by fitting the entropy density to the results from the PNJL model [175]. The resulting parametrization for the 'critical' coupling constant is given by

$$
\alpha_{S}^{c r i t}=a \cdot\left(T / T_{c}\right)^{-12}
$$

where $a=\alpha_{S}^{\text {cross }}\left(T=T_{C E P}\right)$.

The total coupling constant $\alpha_{S}^{D Q P M-C P}$ then reads

$$
\alpha_{S}^{D Q P M-C P} \equiv\left\{\begin{array}{l}
\mu_{B}=\mu_{C E P}: \quad \alpha_{S}^{C E P}=\frac{1-F(T)}{2} \alpha_{S}^{\text {crit }}+\frac{1+F(T)}{2} \alpha_{S}^{\text {cross }} . \\
\mu_{B} \neq \mu_{C E P}: \quad \alpha_{S}^{\text {cross }}
\end{array}\right.
$$

Here $\alpha_{S}^{\text {cross }}$ denotes the coupling constant for the crossover region defined by Eq. (167), while at $\mu_{B}=\mu_{C E P}=0.096 \mathrm{GeV}$ the effective coupling $\alpha_{S}^{D Q P M-C P}=\alpha_{S}^{C E P}$ includes the 


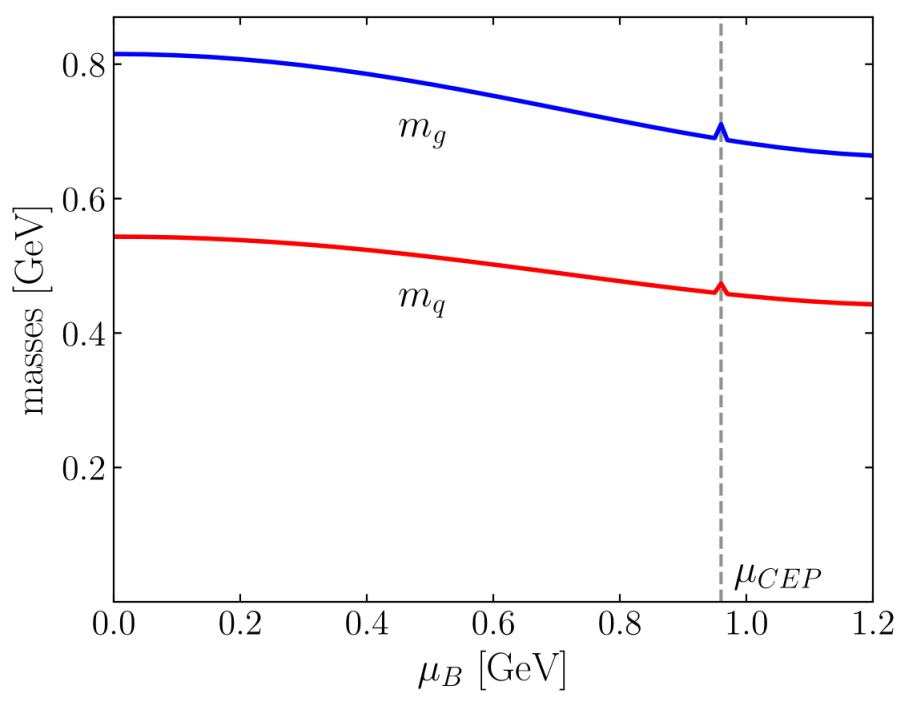

Figure 5.5: Effective masses of (light) quarks and gluons in the DQPM-CP along the critical line (given by Eq. (166)) as a function of the baryon chemical potential $\mu_{B}=3 \mu_{q}$. The dashed line represents the critical value of the baryon chemical potential $\mu_{C E P}=0.96$ $\mathrm{GeV}$.

additional 'critical' contribution $\alpha_{S}^{c r i t}$, defined by Eq. (169). To match the two coupling constants we employ the smoothing function:

$$
F(T)=\tanh \left[\frac{T-0.1004}{\delta T}\right]
$$

where $\delta T=0.002 \mathrm{GeV}$ is the region in the vicinity of the CEP, where the two coupling constants have to match.

The effective coupling of the DQPM-CP is depicted in Fig. 5.4 for fixed baryon chemical potentials: $\mu_{B}=0$ (black solid line), $\mu_{B}=0.6 \mathrm{GeV}$ (green solid line) and $\mu_{B}=0.96 \mathrm{GeV}$ (red points with dashed line) as a function of the scaled temperature $T / T_{c}\left(\mu_{B}\right)$. At vanishing chemical potential the coupling constant coincides with the DQPM effective coupling $g^{2}\left(T, \mu_{\mathrm{B}}=0\right)$ presented in Chapter 2. The scaling behavior of the quasiparticle masses has been widely used in condensed matter physics [216]. There the divergent behaviour of the effective masses of the quasiparticles originates from the interaction with bosonic fluctuations near the critical point. Alternatively, one can include the scaling behavior of the thermodynamic quantities in a more rigorous way as done in Ref. [217], where the EoS from the 1QCD calculations of the BMW collaboration has been parametrized and adopted to include a singular part near the CEP from the 3D-Ising model. Figure 5.4 shows the effective coupling of the DQPM-CP at fixed $\mu_{B}=0$ (red solid line) and $\mu_{B}=0.96 \mathrm{GeV}$ (green points) as a function of the scaled temperature $T / T_{c}$. At $\mu_{B}=0$ the coupling constant is identical to the DQPM effective coupling $g^{2}\left(T, \mu_{\mathrm{B}}=0\right)$ obtained from Eq. (28).

The effective masses of quarks and gluons along the critical line are depicted in Fig. 5.5. The masses decrease with increasing of $\mu_{B}$, while at the CEP they show a peak, which 
corresponds to the finite value of the 'critical' coupling constant.

In a simplified picture, the critical behaviour of the effective masses of partonic quasiparticles near the CEP $\left(T>T_{C E P}\right)$ can be interpreted as the energy density contained in a volume $V \sim \xi_{T}^{3}$ defined by the correlation length (as well as the static exponents and scaled temperature). The energy density, when approaching the CEP, manifests the critical scaling, which can be expressed through the static exponent $\epsilon \sim(t-1)^{1-\alpha}$, and the masses will, correspondingly, depend on the correlation length as well as $\xi_{T} \sim(t-1)^{-\nu}$. Hence the 'critical' contribution to the masses of quasiparticles should increase, approaching the CEP from the partonic side, as $m \sim(t-1)^{1-\alpha-3 \nu} \sim(t-1)^{-1}$.

It is important to note that in DQPM-CP the quark masses along the phase transition line are larger than a third of the free proton mass. This implies that the production of baryons across the critical line, the dominant process for large $\mu_{B}$ and small $T$, is an exothermic process in DQPM-CP.

\subsection{Thermodynamic observables for finite temperature and chemical potential}

In this Section we consider the basic thermodynamic observables from the DQPM-CP for finite chemical potential. The starting point for the calculation of the thermodynamic functions in the dynamical quasiparticle models is the evaluation of the entropy density and the quark densities via the propagators as described in Section 3 Eqs. (36) and (14). Then we can deduce the pressure $p$ (and later the energy density), by the integration of the baryon density over the baryon chemical potential:

$$
p\left(T, \mu_{\mathrm{B}}\right)=p_{0}(T, 0)+\int_{0}^{\mu_{\mathrm{B}}} n_{B}\left(T, \mu_{\mathrm{B}}^{\prime}\right) d \mu_{\mathrm{B}}^{\prime} .
$$

For the pressure at $\mu_{B}=0$ we use the lQCD parametrization of the pressure $p_{0}(T, 0)$ provided by the BMW collaboration in Ref. $[22,60]$. The energy density $\epsilon$ then follows from the Euler relation

$$
\epsilon=T s-p+\sum_{i} \mu_{i} n_{\mathrm{i}}
$$

Furthermore, the interaction measure is defined as:

$$
I \equiv \epsilon-3 P=T s-4 p+\sum_{i} \mu_{i} n_{\mathrm{i}}
$$

which vanishes in the non-interacting limit of massless degrees of freedom at $\mu_{\mathrm{B}}=0$. The scaled pressure, entropy density and energy density of the QGP phase are supposed to increase with the temperature. However, IQCD calculations of the thermodynamic observables show [218], that the massless non-interacting limit can not be reached even at temperatures of $T \sim 1 \mathrm{GeV}$.

We consider two setups for the quark chemical potentials: (I) $\mu_{q}=\mu_{u}=\mu_{s}=\mu_{B} / 3$ 

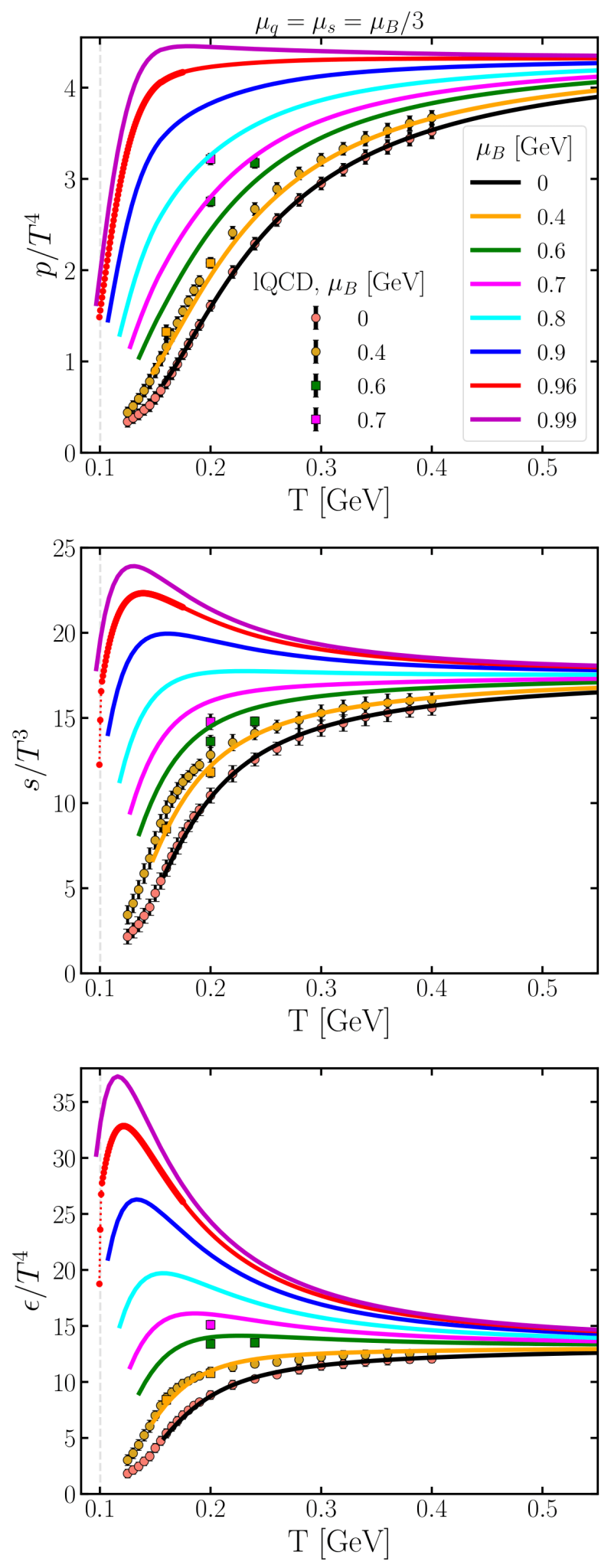

Figure 5.6: Scenario: $\mu_{q}=\mu_{u}=\mu_{s}=\mu_{B} / 3$. From top to bottom: Scaled entropy density $s / T^{3}$, pressure $p / T^{4}$ and scaled energy density $\epsilon / T^{4}$ from the DQPM (lines) as a function of temperature $T$ for various values of $\mu_{\mathrm{B}}[\mathrm{GeV}]$. The lQCD results obtained by the BMW group are taken from Refs. [22,60] (circles) and from Ref. [25] (squares).

and (II) $\mu_{s}=0, \mu_{u}=\mu_{B} / 3$. The quark chemical potential can be related to the strange, baryon and electric charge chemical potentials as $\mu_{i}=B_{i} \mu_{\mathrm{B}}+Q_{i} \mu_{\mathrm{Q}}+S_{i} \mu_{\mathrm{S}}$, where $B_{i}, Q_{i}$ 
5. Transport properties of the QGP in the dynamical quasiparticle model with a CEP
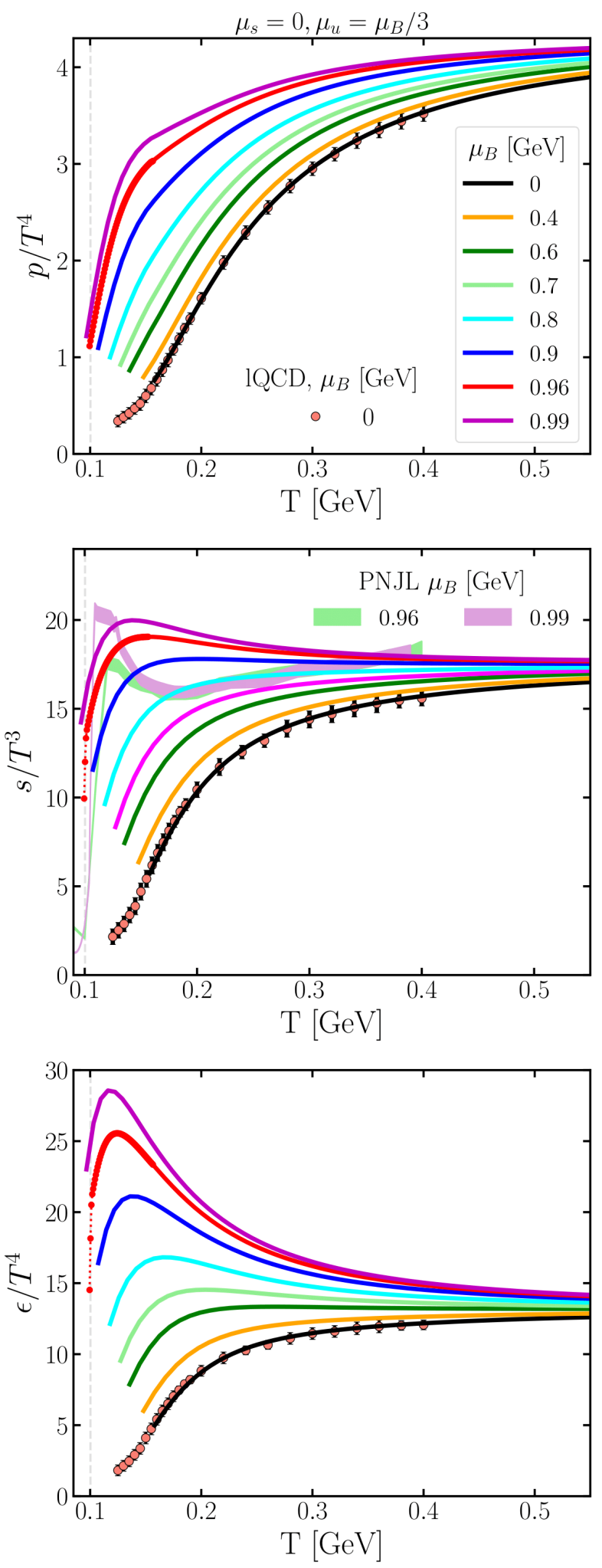

Figure 5.7: Scenario: $\mu_{s}=0, \mu_{u}=\mu_{B} / 3$. From top to bottom: scaled entropy density $s / T^{3}$, pressure $p / T^{4}$ and scaled energy density $\epsilon / T^{4}$ from the DQPM-CP (lines) as a function of temperature $T$ for various values of $\mu_{\mathrm{B}}[\mathrm{GeV}]$. The 1QCD results obtained by the BMW group are taken from Refs. [22,60] (circles). The PNJL results for the entropy density (colored areas) are taken from Ref. [175]. 
and $S_{i}$ are baryon number, electric charge and strangeness of the considered quark. Herein we fix $\mu_{Q}=0$, therefore for the symmetric QGP matter (I) $\mu_{q}=\mu_{u}=\mu_{s}=\mu_{B} / 3$ the strange and the electric charge potentials are vanishing $\mu_{S}=\mu_{Q}=0$, while for (II) $\mu_{s}=0, \mu_{u}=\mu_{B} / 3$ the strange chemical potential is finite $\mu_{S}=\mu_{B} / 3$.

Main thermodynamic observables, such as the scaled entropy density, the pressure and the energy density from the DQPM-CP are displayed Fig. $5.6\left(\mu_{q}=\mu_{u}=\mu_{s}=\mu_{B} / 3\right)$ and Fig. $5.7\left(\mu_{s}=0, \mu_{u}=\mu_{B} / 3\right)$ as a function of temperature for various baryon chemical potentials $0 \leq \mu_{B} \leq 0.99 \mathrm{GeV}$. For setup (I) we find a good agreement between the DQPM$\mathrm{CP}$ results (lines) and results from $1 \mathrm{QCD}$, obtained by the BMW group $[22,60]$ at $\mu_{\mathrm{B}}=0$ and $\mu_{\mathrm{B}}=400 \mathrm{MeV}$. The thermodynamical quantities increase with $\mu_{B}$. When approaching the $\mathrm{CEP}$ at $\mu_{B}=0.96 \mathrm{GeV}$ the values of the entropy density, of the energy density as well as of the quark or baryon density rise suddenly.

For setup (II) we compare the results for the entropy density to that of the Nantes PNJL approach [175]. The DQPM-CP results are in agreement with the PNJL results in the high temperature region $T \geq 0.3 \mathrm{GeV}$, while in the vicinity of the phase transition there is a clear deviation from the PNJL results, which can be expected since the two models encompass different microscopic properties of the degrees of freedom. The resulting values of the thermodynamic observables for setup (II) is smaller than for setup (I) since the contribution from the strange quarks to the quasiparticle entropy density (see Eq. (36)) is smaller for $\mu_{s}=0$ essentually due to the derivatives $\frac{\partial f_{q}\left(\omega-\mu_{q}\right)}{\partial T}$.

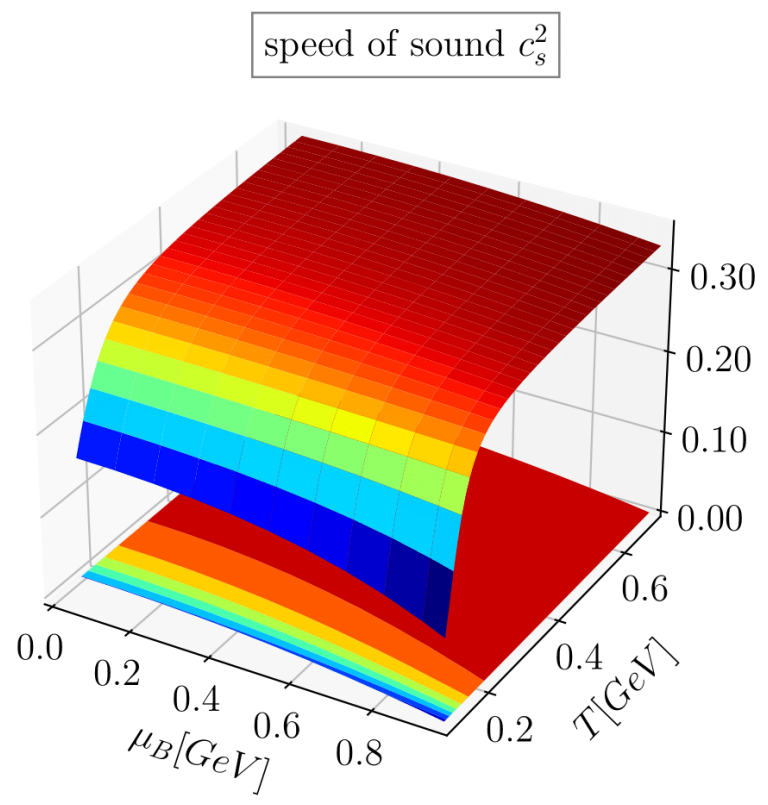

Figure 5.8: Scenario: $\mu_{q}=\mu_{u}=\mu_{s}=\mu_{B} / 3$. The speed of sound squared $c_{s}^{2}$ from the DQPM-CP for a crossover phase transition $\left(0 \leq \mu_{B}<0.96\right)$ as a function of $T$ and $\mu_{B}$. 
5. Transport properties of the QGP in the dynamical quasiparticle model with a CEP

(a) speed of sound $c_{s}^{2}$ for $\mu_{B}=0$

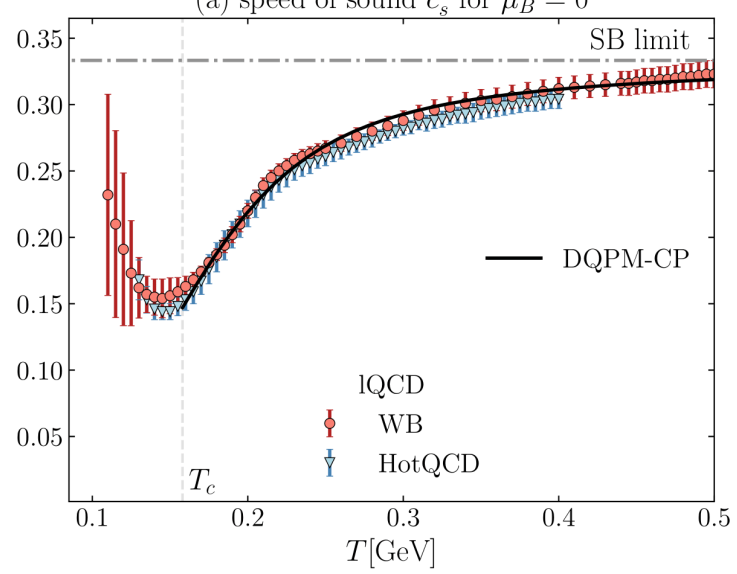

(b) speed of sound $c_{s}^{2}$ for $\mu_{B} \geq 0$

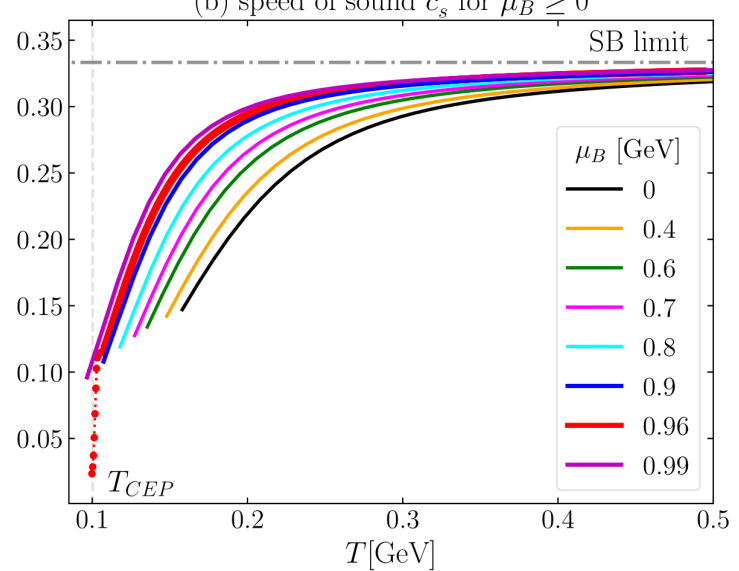

Figure 5.9: Scenario: $\mu_{q}=\mu_{u}=\mu_{s}=\mu_{B} / 3$. The speed of sound squared $c_{s}^{2}$ from the DQPM-CP for (a) $\mu_{B}=0$ and (b) $\mu_{B} \geq 0$ as a function of $T$ compared to lQCD results for $\mu_{B}=0$ obtained by the Wuppertal-Budapest collaboration [22](light red circles) and the HotQCD collaboration [72] (blue triangles).

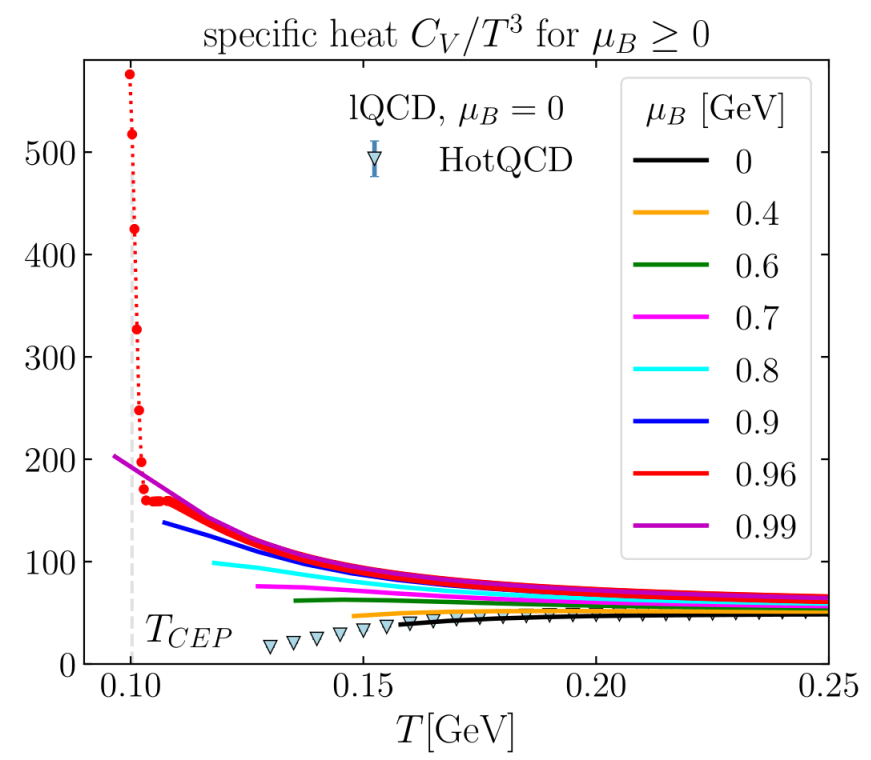

Figure 5.10: Scenario: $\mu_{q}=\mu_{u}=\mu_{s}=\mu_{B} / 3$. The specific heat $C_{V} / T^{3}$ from the DQPM$\mathrm{CP}$ at fixed $\mu_{B}$ as a function of $T$ compared to lQCD results for $\mu_{B}=0$ of the HotQCD collaboration [72].

\subsection{Approaching the CEP from the deconfined phase}

To realize a critical behaviour of the thermodynamic observables in the vicinity of the CEP we introduce, as described above, the 'critical' contribution to the coupling constant that affects the microscopic and macroscopic quantities. At the CEP, where the transition is of second order, the entropy density and baryon density increase rapidly but remain finite, while the quark susceptibility and the specific heat $C_{V} / T^{3}=\frac{d \epsilon}{d T}$ diverge. Therefore, the speed of sound (squared) vanishes as one approaches the CEP. We consider the speed of sound and the specific heat at fixed $\mu_{B}$. For fixed $\mu_{B}$ the speed of sound can be expressed 
as:

$$
c_{s}^{2}=\frac{d p}{d \epsilon}=\frac{d p / d T}{d \epsilon / d T}=\frac{s}{C_{V}} .
$$

To illustrate the $T$ - and $\mu_{B}$-dependence of the speed of sound (squared) for the QGP we show resulting $c_{s}^{2}$ from the DQPM-CP model in Fig. 5.8 as a function of temperature $T \geq T_{c}\left(\mu_{B}\right)$ and baryon chemical potential $\mu_{B}$ in the crossover region, where $\mu_{B} \leq 0.95$ $\mathrm{GeV}$. As one can see from the figure $c_{s}^{2}$ increases with temperature and decreases near the phase transition with increasing $\mu_{B}$. This trend is in agreement with the expectations from the 1QCD calculations for moderate baryon chemical potentials $\mu_{B} \leq 0.4 \mathrm{GeV}$, where the speed of sound has slight decrease with $\mu_{B}$ near the phase transition region [60].

A closer comparison of the DQPM-CP results for $c_{s}^{2}$ at $\mu_{B}=0$ with the available 1QCD estimations from the Wuppertal-Budapest collaboration [22] (light red circles) and the HotQCD collaboration [72] (blue triangles) is provided in Fig. 5.9 (a). The DQPM$\mathrm{CP}$ results are in agreement with the lattice QCD predictions within the estimated errors. Figure 5.9 (b) displays the speed of sound squared $c_{s}^{2}$ from the DQPM-CP as a function of the temperature for a wide range of baryon chemical potentials, including the vicinity of the CEP. At high temperatures the values of $c_{s}^{2}$ are approaching the limit of a non-interacting gas of massless quarks and gluons (SB limit, grey dash-dotted line) $c_{s}^{2}(S B)=1 / 3$. When increasing the baryon chemical potential the speed of sound near the transition temperature decreases, while at the CEP the speed of sound shows a sharp decrease.

Another important quantity the scaled specific heat $C_{V} / T^{3}$ is shown in Fig. 5.10 as a function of $T$ for fixed chemical potentials. As opposed to the $T$-dependence of the speed of sound, the specific heat increases when approaching the phase transition. For moderate values of the baryon chemical potential $\mu_{B}$ the scaled specific heat increases moderately with decreasing temperature. As it approaches the CEP, $C_{V} / T^{3}$ diverges as a function of $T$, which is consistent with the expectations for a second-order phase transition.

The resulting $T$-dependence of the specific heat for the critical baryon chemical potential $\mu_{B}=0.96 \mathrm{GeV}$ near the CEP allows to estimate the critical exponent for $T>T_{C E P}$ :

$$
\ln \left(C_{V}\right)=-\alpha \cdot \ln \left(T-T_{C E P}\right)+\text { const }
$$

For the presented parametrization of the coupling constant we obtain the following values: $\alpha=0.63 \pm 0.02$ and const $=-5.48 \pm 0.01$. The value of the critical exponent $\alpha$ is in agreement with the predictions from the PNJL model for $T>T_{C E P}: \alpha_{P N J L}=0.68 \pm 0.01$ [191] and the expectations from the universality argument $\alpha=2 / 3$ in Ref. [219].

In the case of $\mathrm{QCD}$ with finite quark masses both the chiral and center symmetries are explicitly broken. What remains is the $Z(2)$ sign symmetry of the order parameter of the chiral phase transition. Therefore it has been assumed that the CEP of QCD with finite quark masses belongs to the three-dimensional $Z(2)$ Ising universality class [220-222]. The corresponding critical exponent in the $Z(2)$ universality class is $\alpha \approx 0.11$ [223]. However, it is known that the critical exponents in the PNJL(NJL) model and $Z(2)$ universality class differ $[191,204,224-226]$. To explore the high density region, it is essential for effective 

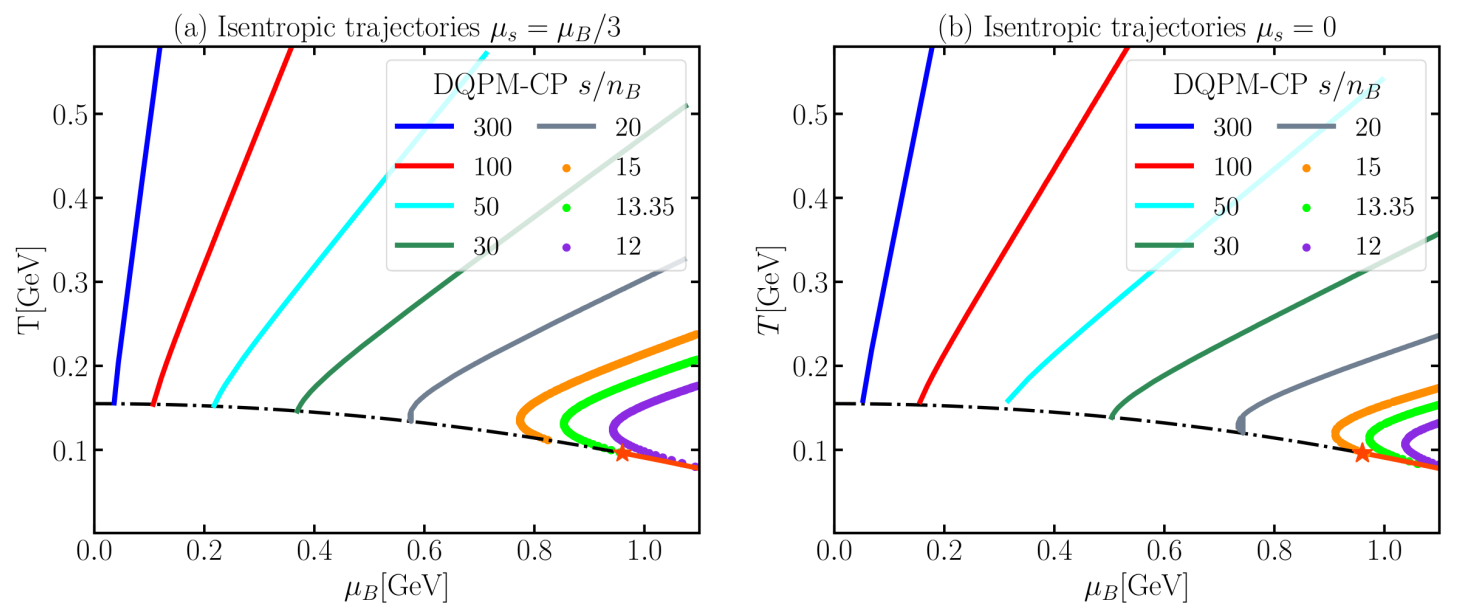

(c) Isentropic trajectories

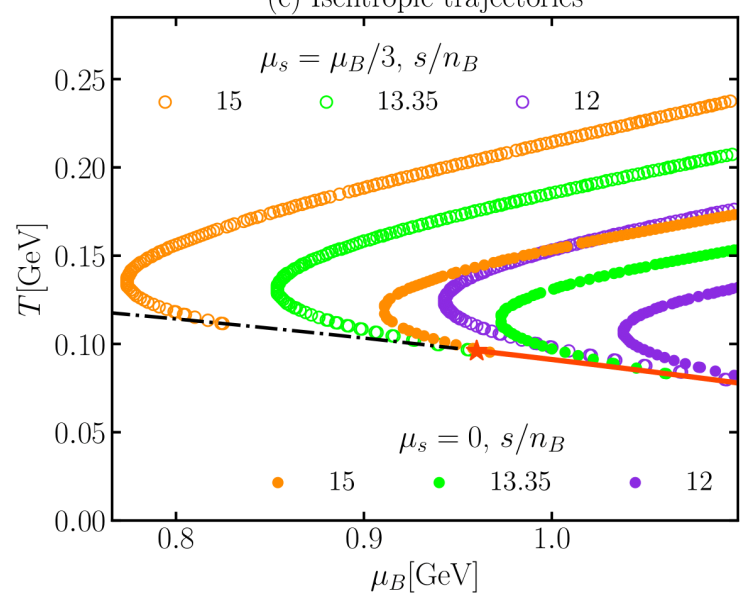

Figure 5.11: Trajectories of constant $s / n_{B}$ in the DQPM-CP phase diagram for $T>T_{c}$ and (a) $\mu_{q}=\mu_{u}=\mu_{s}=\mu_{B} / 3$, (b) $\mu_{s}=0, \mu_{u}=\mu_{B} / 3$ and (c) two cases in the vicinity of the critical endpoint CEP. The finite temperature crossover (black dash-dotted line) at small chemical potential switches to the large chemical potential first-order transition (red solid line) at the CEP (star), which is located at $(0.10,0.96) \mathrm{GeV}$. 
models to consider isentropic trajectories for which the ratio of entropy to baryon number is held constant. The isentropic trajectories correspond to an ideal hydrodynamical expansion of QGP matter as created in the HICs. Futhermore, the dissipative effects, which can be described by the viscosities and diffusion coefficients, alter the trajectories [227,228]. The presence of the CEP can affect the isentropic trajectories, since the entropy density and baryon density undergo a rapid change as the phase transition is approached. It is supposed that the CEP acts as an attractor of isentropic trajectories [229]. Moreover, a different choice for the strange quark chemical potential affects the trajectories as well. Therefore we compare the resulting isentropic trajectories for two setups of the strange chemical potential.

Figure 5.11 displays the isentropic trajectories from the DQPM-CP for (a) $\mu_{q}=\mu_{s}=$ $\mu_{u}=\mu_{B} / 3$ and (b) $\mu_{s}=0, \mu_{u}=\mu_{d}=\mu_{B} / 3$ in the phase diagram. Comparing (a) to (b) one can clearly see that the trajectories for the zero strange quark chemical potential are shifted towards higher $\mu_{B}$ values. In the case of vanishing chemical potential of the strange quark $\mu_{s}=0, \mu_{u}=\mu_{d}=\mu_{B} / 3$, the entropy density, which has also contributions from the light (anti-)quarks and gluons, is less affected than the baryon density. Therefore, for finite $\mu_{B}>0$ and $\mu_{s}=0$ the ratio $s / n_{B}$ is larger than in the case of a symmetric setup $\mu_{s}=\mu_{u}=\mu_{B} / 3$ and the value of the baryon density decreases faster than the entropy density. This observation is in agreement with previous studies of the PNJL model [230] and the results from Ref. [217,231], where the 1QCD EoS from the WB collaboration $[22,60,232]$ with a critical point in the 3D Ising model universality class is considered for moderate baryon chemical potentials $\mu_{B} \leq 0.45 \mathrm{GeV}$. Thus, a 'critical' trajectory, which goes through the CEP, for (a) corresponds to $s / n_{B} \approx 13.35$, for (b) corresponds to $s / n_{B} \approx 15$. Furthermore, a direct comparison of isentropic trajectories in a vicinity of the CEP is displayed in Fig. 5.11 (c). In the vicinity of the CEP, the trajectories with $s / n_{B}=15,13.35,12$ shown in Fig. 5.11(c) are focused to the critical endpoint.

\subsection{Transport coefficients of the QGP near the CEP}

We continue to investigate the transport properties of QGP matter using the DQPM-CP. We consider the specific shear $\eta / s$ and bulk $\zeta / s$ viscosities, the ratio of electric $\sigma_{Q Q} / T$, baryon $\sigma_{B B} / T$ and strange $\sigma_{S S} / T$ conductivities to temperature. At vanishing baryon chemical potential the DQPM-CP model equals the DQPM, therefore one can find the comparison of DQPM transport coefficients at $\mu_{B}=0$ with the recent results from various approaches in previous papers $[1,2,5]$.

\subsubsection{Specific viscosities}

The shear and bulk viscosity for quasiparticles with medium-dependent masses $m_{i}\left(T, \mu_{q}\right)$ can be derived using the Boltzmann equation in the RTA [74] through the relaxation time:

$$
\eta\left(T, \mu_{q}\right)=\frac{1}{15 T} \sum_{i=q, \bar{q}, g} \int \frac{d^{3} p}{(2 \pi)^{3}} \frac{\mathbf{p}^{4}}{E_{i}^{2}} \tau_{i}\left(\mathbf{p}, T, \mu_{q}\right) \times d_{i}\left(1 \pm f_{i}\right) f_{i},
$$




$$
\zeta\left(T, \mu_{q}\right)=\frac{1}{9 T} \sum_{i=q, \bar{q}, g} \int \frac{d^{3} p}{(2 \pi)^{3}} \tau_{i}\left(\mathbf{p}, T, \mu_{q}\right) \frac{d_{i}\left(1 \pm f_{i}\right) f_{i}}{E_{i}^{2}}\left[\mathbf{p}^{2}-3 c_{s}^{2}\left(E_{i}^{2}-T^{2} \frac{d m_{i}^{2}}{d T^{2}}\right)\right]^{2},
$$

where $q(\bar{q})=u, d, s(\bar{u}, \bar{d}, \bar{s}), d_{q}=2 N_{c}=6$ and $d_{g}=2\left(N_{c}^{2}-1\right)=16$ are the degeneracy factors for spin and color for quarks and gluons, respectively, whereas $\tau_{i}$ are the relaxation times. $c_{s}$ is the speed of sound for a fixed $\mu_{B}$ given by Eq. (175), $\frac{d m_{i}^{2}}{d T^{2}}$ are the derivative of the effective masses.
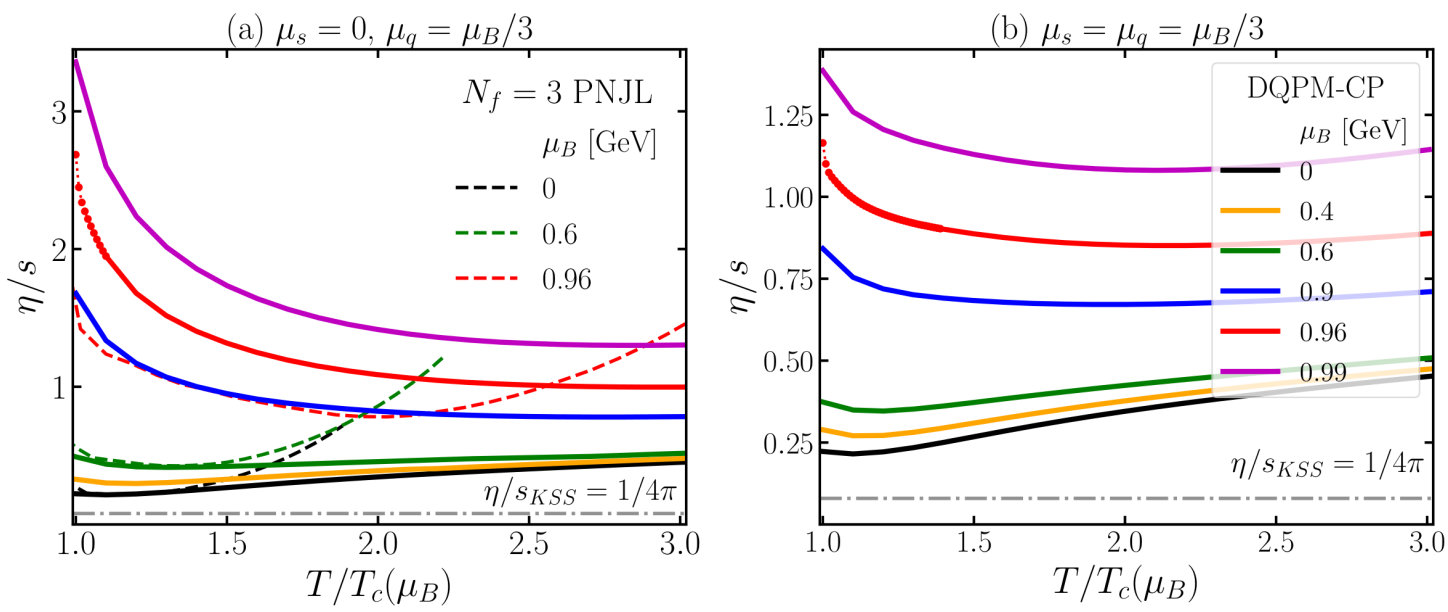

Figure 5.12: Specific shear $\eta / s$ viscosity from the DQPM-CP (solid lines) for two setups of the strange chemical potential: (a) $\left(\mu_{s}=0, \mu_{u}=\mu_{B} / 3\right)$ and (b) $\left(\mu_{s}=\mu_{u}=\mu_{B} / 3\right)$ as a function of the scaled temperature $T / T_{C}$ for various $\mu_{B} \geq 0$. We compare to the RTA estimates from the $N_{f}=3$ PNJL model (dashed lines) [5]. The grey dashed-dotted line demonstrates the Kovtun-Son-Starinets bound [99] $(\eta / s)_{\mathrm{KSS}}=1 /(4 \pi)$.
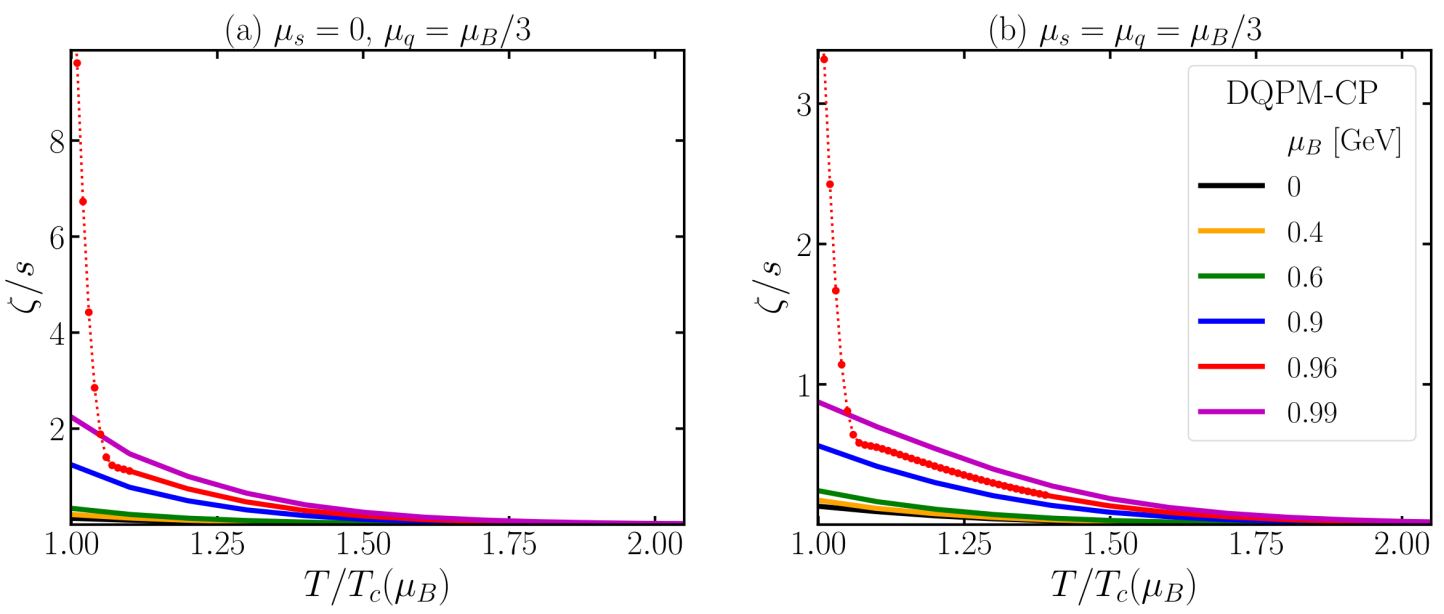

Figure 5.13: Specific bulk $\zeta / s$ viscosity from the DQPM-CP (solid lines) for two setups of the strange chemical potential: (a) $\left(\mu_{s}=0, \mu_{u}=\mu_{B} / 3\right)$ and (b) $\left(\mu_{s}=\mu_{u}=\mu_{B} / 3\right)$ as a function of the scaled temperature $T / T_{c}$ for various $\mu_{B} \geq 0$.

As it was shown in the previous studies $[1,2,81,196,233]$ in case of the medium dependent masses, the viscosities display a pronounced temperature behaviour. At vanishing 
baryon chemical potential we found previously that the DQPM results for specific shear and bulk viscosity $[1,5]$ are very close to the predictions from the gluodynamic $1 Q C D$ calculations $[85,87]$.

For moderate baryon chemical potentials, the specific shear viscosity $\eta / s$ of the QGP matter increases with temperature, while the specific bulk viscosity $\zeta / s$ decreases with temperature, independent of the baryon chemical potential. However, the $T$-dependence of $\eta / s$ near the phase transition changes with increasing $\mu_{B}$. Fig. 5.12 shows the DQPMCP results for $\eta / s$ as a function of the scaled temperature $T / T_{c}\left(\mu_{B}\right)$, for different $\mu_{B}$. The specific shear viscosity for $\mu_{B}=0$ (red line) shows a dip followed by an increase with temperature while for $\mu_{B} \geq 0.9 \mathrm{GeV} \eta / s$ decreases with increasing temperature near the phase transition $\left(T \leq 2 T_{c}\right)$. The parton relaxation time $\tau_{i}$ decreases with increasing temperature at lower $\mathrm{T}$, and remains approximately constant at high $T$ for moderate values of chemical potential $\mu_{B} \geq 0.6 \mathrm{GeV}$. Therefore the shear viscosity $\eta \sim T^{4}$, while the entropy density grows as $s \sim T^{3}$. Thus, in the high temperature region the ratio $\eta / s$ increases as $\sim T$. It is important to note that transport coefficients rely on the microscopic properties of the degrees of freedom. While a variety of the models can reproduce the $1 Q C D$ results of basic thermodynamic observables, the transport coefficients differ between the models.

Here we compare results for the specific shear viscosity and later for the electric conductivity for non-zero baryon or quark chemical potential with the RTA results from the PNJL model for $\mu_{s}=0, \mu_{u}=\mu_{B} / 3$ in Fig. 5.12 (a). The specific shear viscosity results from the DQPM-CP (solid lines) for $\mu_{B}=0,0.6 \mathrm{GeV}$ agree well with the predictions of the PNJL model (dashed lines) in the vicinity of the phase transition for temperatures $T \leq 1.5 T_{c}$. The DQPM-CP results for $\eta / s$ at $\mu_{B}=0.96 \mathrm{GeV}$ for $T \leq 2 T_{c}$ is higher than from the PNJL model, while the temperature dependence is similar. The difference between the results is caused by the different treatment of the gluonic degrees of freedom, which has a pronounced critical behaviour of the thermal masses in the DQPM-CP model. Increasing the baryon chemical potential, one can see not only an increase in magnitude but also a change in the $T$-dependence of $\eta / s$ and $\zeta / s$ as shown in Fig. 5.12.

In particular in the vicinity of the phase transition $T<1.5 T_{c}$ for moderate values of $\mu_{q}$ the specific shear viscosity shows a dip after the phase transition, which is vanishing at high values of $\mu_{B}$ as can be seen in Fig. 5.12.

As pointed out in Refs. [102, 196,234,235] in the vicinity of the CEP, the divergences of bulk and shear viscosities of the QCD matter are determined by the dynamic and the static critical exponents. The dynamical universality class of the QCD critical endpoint is argued to be that of the H-model $[102,207]$ according to the classification of dynamical critical phenomena by Hohenberg and Halperin [208]. Whereas in the vicinity of the CEP the shear viscosity has a mild divergence in the critical region, the bulk viscosity has a more pronounced divergence [102,208,209]: $\eta \sim \xi_{T}^{Z_{\eta}}\left(Z_{\eta} \approx 1 / 19\right), \zeta \sim \xi_{T}^{Z_{\zeta}}\left(Z_{\zeta} \approx 3\right)$. The thermal correlation length is controlled by the static critical exponent $\xi_{T} \sim(r)^{-\nu}, r=t-1=\frac{T-T_{c}}{T_{c}}$ denotes the reduced tempearture, with $\nu$ being the static critical exponent. Using the hyperscaling relation [203] for the static critical exponents we can estimate $\nu$ :

$$
2-\alpha=d \nu
$$


where $d=3$ denotes the number of the spatial dimensions, $\alpha \approx 0.63$. We obtain $\nu \approx 0.46$. Taking into account the dynamical and static exponents the divergence of the bulk viscosity is assumed to be $\zeta \approx r^{-Z_{\zeta} \nu+\alpha}[209,235,236]$.

Here we consider small deviations from equilibrium where the quark relaxation times are not large: $\tau_{q}$ is about $4.5-2.5 \mathrm{fm} / \mathrm{c}$ for the temperature range $T_{c}<T \leq 2 T_{c}$, so that the slight divergence of the transport coefficients near the CEP is determined by the static exponents. The specific shear and bulk viscosities from the DQPM-CP increases rapidly approaching the the critical endpoint from the partonic phase. The increase near the CEP is more pronounced for the bulk viscosity, which is related to the rapid decrease in the speed of sound and corresponds to the static critical exponents, that affects the bulk viscosity. In terms of heavy-ion collisions observables, this increase in the bulk viscosity is expected to show up as the decrease of average transverse momentum of produced particles as well as in an increase of the charged particle multiplicity per unit momentum rapidity $[42,234]$. However, this has to be checked by transport simulations or by a hydrodynamical simulation of the expanding QGP. Such a substantial increase of the charged particle and net-baryon multiplicities per unit momentum rapidity due to the enhancement of the bulk viscosity near the CEP has been observed in a longitudinally expanding $1+1$ dimensional causal relativistic hydrodynamical evolution at non-zero baryon density [237].

We note that the specific bulk and shear viscosities have been considered near the CEP and the 1 st order phase transition for the $N_{f}=2$ NJL model in a previous study [196]. We found good qualitative agreement for the $T$-dependence of the shear and bulk viscosity of the NJL model from Ref. [196], while the numerical values differ due to the different quark relaxation times and the absence of gluonic degrees of freedom in case of the NJL model.

\subsubsection{Electric, baryon and strange conductivities}
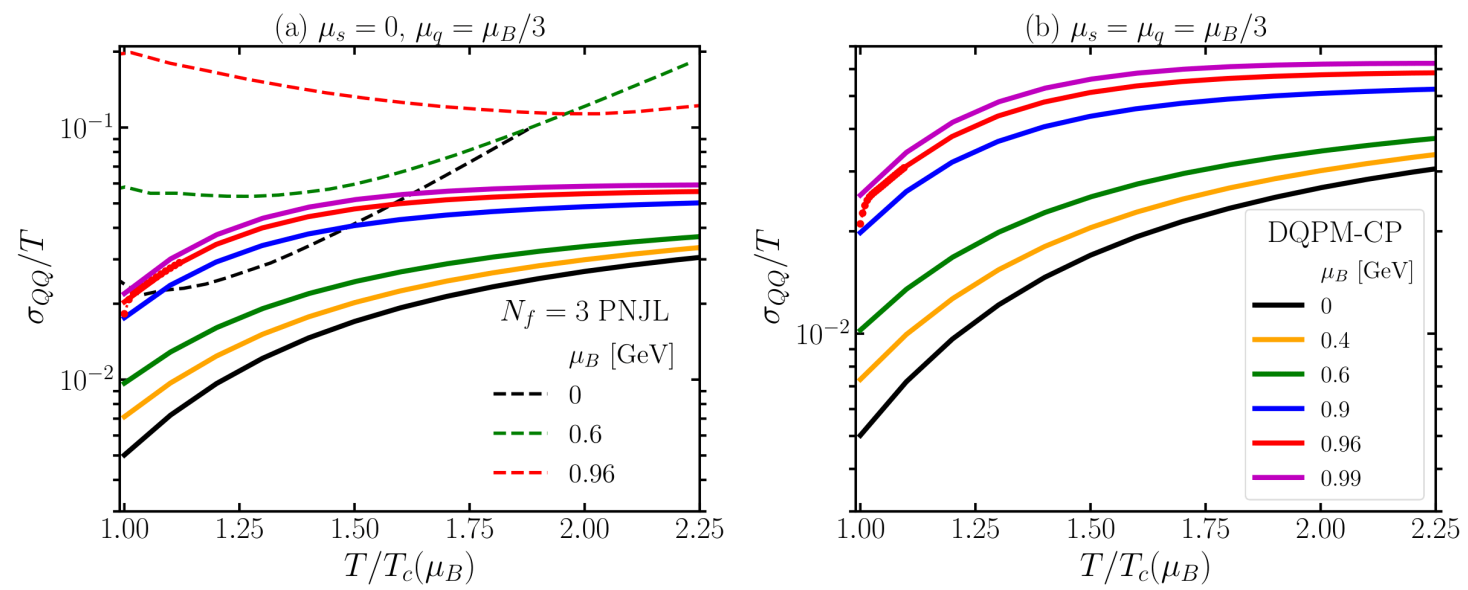

Figure 5.14: Scaled electric conductivity $\sigma_{Q Q} / T$ as a function of the scaled temperature $T / T_{c}$ from the DQPM-CP (solid lines) for two setups of the strange chemical potential: (a) $\left(\mu_{s}=0, \mu_{u}=\mu_{B} / 3\right)$ and (b) $\left(\mu_{s}=\mu_{u}=\mu_{B} / 3\right)$ as a function of the scaled temperature $T / T_{c}$ for various $\mu_{B} \geq 0$. For $\left(\mu_{s}=0, \mu_{u}=\mu_{B} / 3\right)$ we compare $\sigma_{Q Q} / T$ from DQPM-CP to the RTA estimates from the $N_{f}=3$ PNJL model (dashed lines) [5]. 


\section{Transport properties of the QGP in the dynamical quasiparticle model} with a CEP

In the region of the high net baryon density it is important to take into account the diffusion of conserved charges, i.e. electric, baryon and strange charges, from higher density regions to lower density regions. The transport coefficient, which characterizes the diffusion, is the diffusion coefficient $\kappa_{q}$ or the conductivity $\sigma_{q}=\kappa_{q} / T$ of the conserved charge $q$. Furthermore, since the quarks carry multiple conserved charges, one needs to consider additionally non-diagonal conductivities for two conserved charges $q q^{\prime}-\sigma_{q q^{\prime}}$. Conductivities $\sigma_{q q^{\prime}}$ for quasiparticles can be expressed in the RTA [104] as:

$$
\begin{array}{r}
\sigma q q^{\prime}\left(T, \mu_{q}\right)=\frac{1}{3 T} \sum_{i=q, \bar{q}} q_{i} \int \frac{d^{3} p}{(2 \pi)^{3}} \frac{\mathbf{p}^{2}}{E_{i}^{2}} \tau_{i}\left(\mathbf{p}, T, \mu_{q}\right) \\
\times\left(\frac{E_{i} n_{q^{\prime}}}{\epsilon+p}-q_{i}^{\prime}\right) d_{i}\left(1 \pm f_{i}\right) f_{i}
\end{array}
$$

Let us consider first the diagonal conductivities for electric, baryon and strange charge.
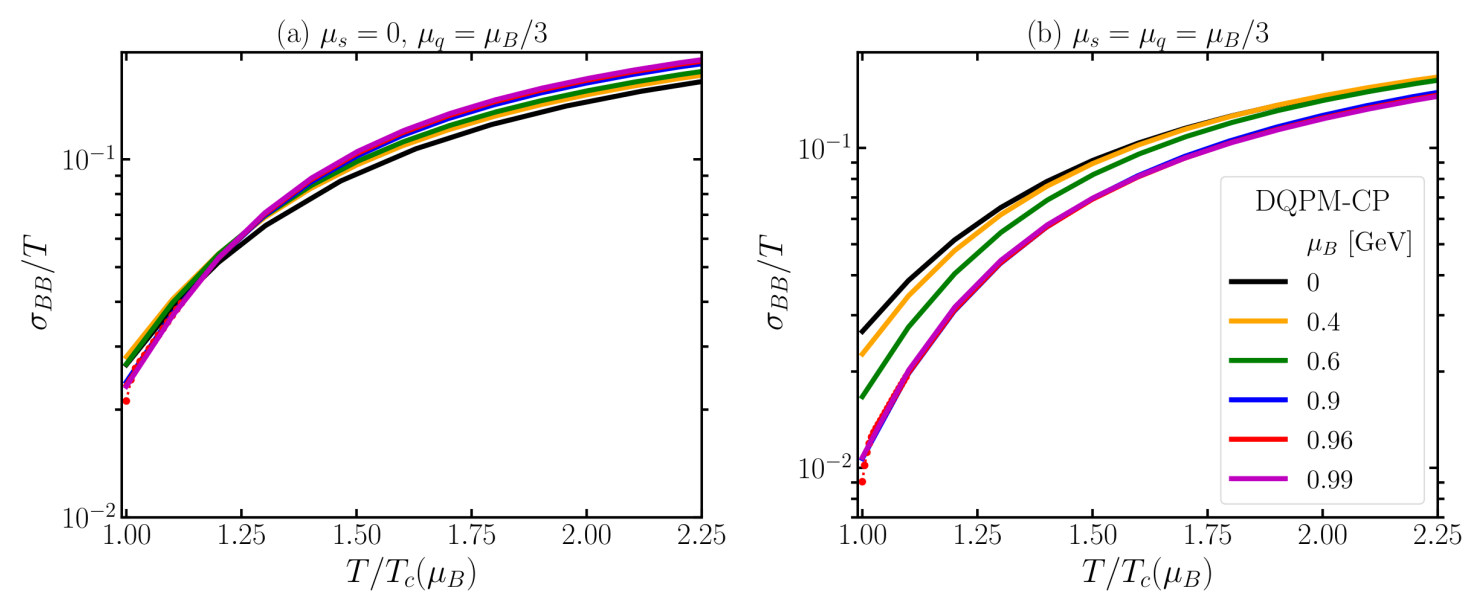

Figure 5.15: Scaled baryon conductivity $\sigma_{B B} / T$ as a function of the scaled temperature $T / T_{c}$ from the DQPM-CP (solid lines) for two setups of the strange chemical potential: (a) $\left(\mu_{s}=0, \mu_{u}=\mu_{B} / 3\right)$ and (b) $\left(\mu_{s}=\mu_{u}=\mu_{B} / 3\right)$ as a function of the scaled temperature $T / T_{c}$ for various $\mu_{B} \geq 0$.

The DQPM-CP results for $\sigma_{Q Q} / T, \sigma_{B B} / T$ and $\sigma_{S S} / T$ are shown in Figs. 5.14, 5.15, 5.16 as a function of the scaled temperature $T / T_{c}$ for two setups of the strange quark chemical potential. The scaled electric, strange and baryon conductivity have a similar temperature dependence: at high $T$ the ratios increase with temperature increase as $\sim T$ which is mainly due to the quark density increasing with temperature. The most prominent difference between the conductivities is the $\mu_{B}$-dependence, which is shown in Figs. 5.14, 5.15, 5.16: the electric and strange conductivities increase with $\mu_{B}$, while the baryon conductivity decreases with $\mu_{B}$ for the symmetric setup $\mu_{s}=\mu_{u}=\mu_{B} / 3$. With the increase of baryon chemical potential the net baryon density increases, which influences the baryon conductivity. A similar trend for $\sigma_{Q Q} / T, \sigma_{B B} / T$ and $\sigma_{S S} / T$ at moderate baryon chemical potentials $\mu_{B} \leq 0.4 \mathrm{GeV}$ has been observed in the non-conformal Einstein-Maxwell-Dilaton (EMD) holographic model [49]. Futhermore, we compare the $\mu_{B}$ dependencies of the scaled conductivities for the two setups of the strange quark chemical potential. We have found that in case of vanishing strange quark chemical potential (setup (II)) the scaled conductivities 

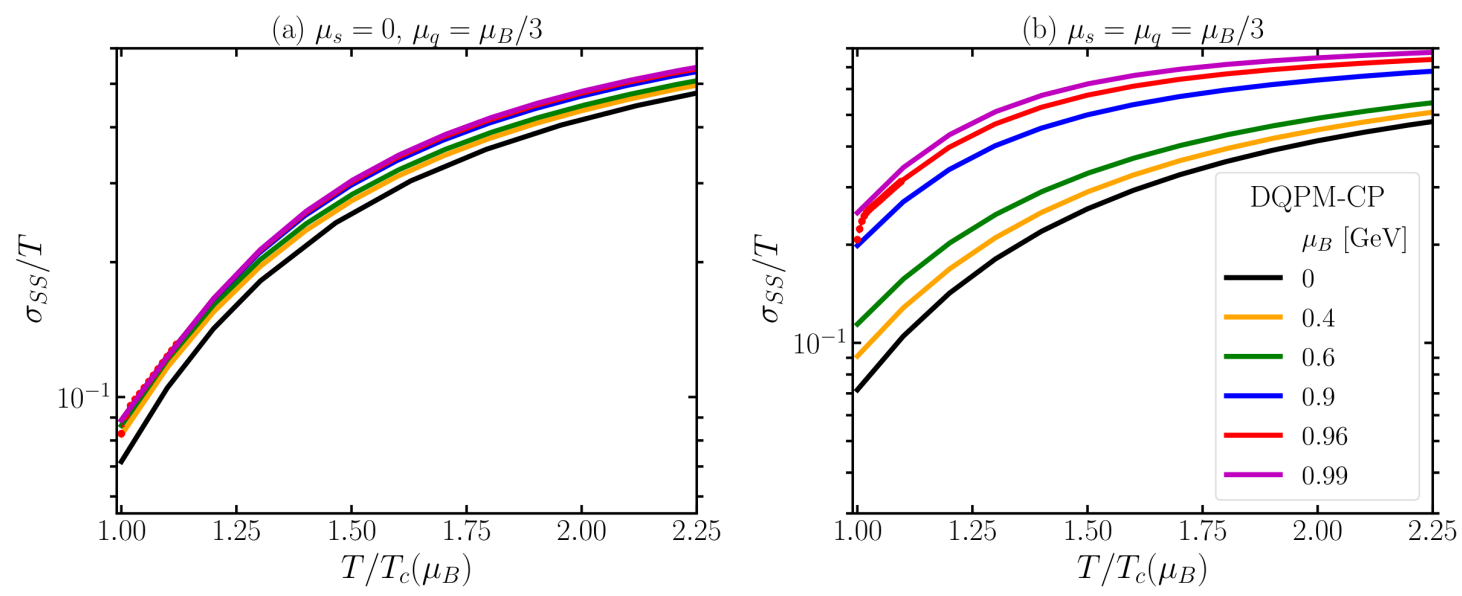

Figure 5.16: Scaled strange conductivity $\sigma_{S S} / T$ as a function of the scaled temperature $T / T_{c}$ from the DQPM-CP (solid lines) for two setups of the strange chemical potential: (a) $\left(\mu_{s}=0, \mu_{u}=\mu_{B} / 3\right)$ and (b) $\left(\mu_{s}=\mu_{u}=\mu_{B} / 3\right)$ as a function of the scaled temperature $T / T_{c}$ for various $\mu_{B} \geq 0$.

show a much less pronounced of $\mu_{B}$-dependence for the baryon and strange conductivities, which is expected due to the vanishing net strangeness density $n_{S}=0$. The electric conductivity has a similar $\mu_{B}$-dependence for the two settings of the strange quark chemical potential. Near the CEP, the electric conductivity decreases, but as for the PNJL results there is no pronounced divergence. The same behavior is found for the baryon and strange conductivities. 


\subsection{Conclusions to Section 5}

We have explored thermodynamic and transport properties of the QGP by the phenomenological extention of the dynamical quasiparticle model to a wide range of baryon chemical potentials. We have adopted the scaling behaviour in the effective coupling, using the the entropy density from the PNJL model near the CEP. The resulting thermodynamic observables agree well with the IQCD data. For moderate values of the chemical potential $\mu_{B} \leq 0.4 \mathrm{GeV}$ the dependence of the thermodynamic quantities on $\mu_{B}$ are in agreement with the previous results from the DQPM $[1,47]$.

- We have presented the results for the thermodynamic observables $p / T^{4}, \epsilon / T^{4}, s / T^{3}$, as well as for the speed of sound and the specific heat for a wide range of chemical potentials. We have shown that the 'critical' behaviour of the effective coupling affects the thermodynamic observables. Moreover, we have found that the resulting value of the critical exponent $\alpha \approx 0.63$ is in good agreement with the predictions of the PNJL model and the expectations $\alpha=2 / 3$ from the universality argument.

- To quantify the $\mu_{B}$-dependence of the bulk observables we have studied the isentropic trajectories of the deconfined QCD medium described by the DQPM-CP for a wide range of baryon chemical potentials, including the vicinity of the CEP.

- We have evaluated transport properties of the deconfined QCD medium for a wide range of baryon chemical potentials within the DQPM-CP: the specific shear $\eta / s$ and bulk $\zeta / s$ viscosity and the ratio of electric $\sigma_{Q Q} / T$, baryon $\sigma_{B B} / T$ and strange $\sigma_{S S} / T$ conductivities to temperature on the basis of the Boltzmann equation in the relaxation time approximation. We have found that the resulting $\mu_{B}$-dependencies of $\eta / s$ and $\sigma_{Q Q} / T$ for the $N_{f}=3$ PNJL model and the DQPM-CP model essentially coincide in the vicinity of the phase transition, while there is a clear difference in the electric conductivity.

- We have found that the DQPM-CP result for the specific bulk viscosity has a rapid increase approaching the CEP from the high temperature region originating from the rapid decrease of the speed of sound $c_{s}^{2} \rightarrow 0$, whereas for the specific shear viscosity and the $B, Q, S$ conductivities there are only small increases, caused mainly by the 'critical' contribution of the effective coupling constant.

Although the extracted results for the transport coefficients are model-dependent, the qualitative picture of the $T$ and $\mu_{B}$-dependence is consistent with expectations from more rigorous approaches. The presented results can be implemented in the hydrodynamic simulations as well as be employed for the partonic part of transport approaches for simulations of HICs. 


\section{Conclusions and Outlook}

In this thesis the transport properties of the deconfined phase of strongly interacting matter at finite $T$ and baryon chemical potential $\mu_{B}$ have been investigated. The influence of a type of a phase transition on thermodynamic and transport properties has been studied within effective models.

To achieve the dynamical description of the strongly interacting QCD matter by means of transport and hydrodynamical approaches, one has first to specify the microscopic properties of the relevant degrees of freedom. Here we have focused on the partonic phase, where the degrees of freedom are strongly interacting quarks and gluons, described by the dynamical quasiparticle model (DQPM), which interprets the EoS in terms of dynamical degrees of freedom, and allows to estimate the cross sections of the corresponding elastic and inelastic reactions, which are essential for the transport evolution. Moreover, on the basis of the microscopic properties such as - effective masses, widths and cross sections the evaluation of the transport coefficients within the kinetic theory has been performed. We have first considered high temperatures $T>T_{c}$ and moderate baryon chemical potentials $\mu_{B} \leq 0.5 \mathrm{GeV}$, where, according to the state-of-the-art 1QCD calculations, the phase transition of the QCD medium is found to be a crossover. We presented the EoS and the transport coefficients of the QGP, such as shear and bulk viscosity, electric and baryon conductivities within the DQPM. Further on, in the region of moderate and high net baryon density it is important to take into account the diffusion of conserved charges. To this aim, we have evaluated the full diffusion coefficient matrix for the baryon (B), strange $(\mathrm{S})$ and electric $(\mathrm{Q})$ charges and compare presented relaxation-time (RTA) method to the Chapman-Enskog (CE) method for the diffusion coefficients. In the considered region of moderate baryon chemical potentials $\mu_{B} \leq 0.5 \mathrm{GeV}$ we find only a weak dependence of the transport coefficients on $\mu_{B}$.

Secondly, the out-of equilibrium study of the QGP evolution during the HICs at BES energies within the off-shell Parton-Hadron-String-Dynamics (PHSD) transport approach has been considered. The PHSD covers the full evolution of the system during HICs, including the partonic phase as well as the phase transition between the hadronic and partonic phases, where the microscopic properties of quarks and gluons are described by the DQPM. The microscopic properties of partonic quasiparticles and their differential cross sections depend not only on temperature $T$ but also on the chemical potential $\mu_{B}$ explicitly. We started with the 'bulk' observables in HICs at various energies - from AGS to top RHIC - for symmetric $\mathrm{Au}+\mathrm{Au}$ and $\mathrm{Pb}+\mathrm{Pb}$ collisions. Further on, we studied the sensitivity of the $\mu_{B}$-dependencies of partonic cross sections and microscopic properties of quasiparticles on the flow coefficients. Overall, we have found only a small sensitivity of the considered observables to the $\mu_{B}$-dependence of parton properties and their interaction cross sections. These practically invisible traces of the influence of baryon density on the partonic phase 
can be explained by the fact that at high energies, where the matter is dominated by the QGP phase, one probes the QGP at a very small baryon chemical potential $\mu_{B}$ in central collisions at midrapidity, whereas at lower energies, where $\mu_{B}$ becomes larger, the fraction of the QGP drops rapidly. Therefore, the final observables are in total prevailed by the hadrons which participated in hadronic rescattering; thus the information about their QGP origin is washed out or lost.

We have demonstrated that the $\mu_{B}$-dependence of QGP interactions is more pronounced in observables for strange hadrons (kaons and especially anti-strange hyperons) as well as for antiprotons. It provides an experimental hint for the search of traces of the hot and dense QGP matter for future experiments at the FAIR and NICA facilities. The results presented here demonstrate that the QCD evolution during HICs can be studied by means of the off-shell transport approach.

Next, in order to examine the domain of high $\mu_{B}$ we have applied methods of kinetic theory to evaluate transport coefficients in the $N_{f}=3$ Polyakov extended Nambu-Jona Lasinio (PNJL) model. We demonstrated that for moderate baryon chemical potentials $\mu_{B} \leq 0.8-0.9 \mathrm{GeV}$, where the phase transition is a rapid crossover, transport coefficients show a smooth $T$-dependence when approaching the phase transition from the region of high $T$. While for large baryon chemical potentials $\mu_{B}>0.96 \mathrm{GeV}$ the presence of a first order phase transition modifies significantly the $T$-dependence of the transport coefficients and a discontinuity can be seen when approaching the phase transition. Moreover, we found that outside the critical region the influence of the critical end-point (CEP) on the evaluated transport coefficients is rather small in comparison to the modification due to a first-order phase transition.

Finally, we have considered a phenomenological extension of the DQPM (DQPM-CP) to a wider range of $\mu_{B}$ by including a region with a possible CEP and first-order phase transition. The EoS, the speed of sound as well as transport coefficients for $T>T_{c}$ are demonstrated for a wide range of $\mu_{B}$ and $T$ employing two settings of the strange quark chemical potentials: (I) $\mu_{q}=\mu_{u}=\mu_{s}=\mu_{B} / 3$ and (II) $\mu_{s}=0, \mu_{u}=\mu_{d}=\mu_{B} / 3$. We have demonstrated the critical behaviour of the speed of sound and the specific heat approaching the CEP from the partonic phase. We have evaluated transport coefficients of the QGP medium for a wide range of baryon chemical potential within the DQPM-CP model: the specific shear $\eta / s$ and bulk $\zeta / s$ viscosities and the ratio of electric $\sigma_{Q Q} / T$, baryon $\sigma_{B B} / T$ and strange $\sigma_{S S} / T$ conductivities to temperature. It has been shown that the resulting $\mu_{B}$-dependencies of $\eta / s$ and $\sigma_{Q Q} / T$ for the $N_{f}=3$ PNJL model and the DQPM-CP essentially coincide in the vicinity of the phase transition, while there is a clear difference in the electric conductivity essentually due to the different partonic degrees of freedom.

The calculations presented in the thesis have shown that transport coefficients can differ among the models, which have similar phase structures and EoS. This discrepancy stems from the difference in the dynamic degrees of freedom. It demonstrates that the description of the QGP in terms of thermodynamic observables and the knowledge of the 
phase diagram is not sufficient to describe the dynamical evolution of strongly interacting matter in a unique way.

The qualitative study on transport coefficients and the phenomenological model, which describes the QGP phase for a wide range of baryon densities, developed in this thesis might be useful for hydrodynamical simulations as well as for the development of transport approaches, which include the evolution of the partonic phase (such as the PHSD). The proposed model can be especially useful for the further examination of the region of the strongly interacting matter phase diagram by the simulations of HICs for the moderate and high $T$ and $\mu_{B}$. 


\section{List of Figures}

1.1 The running coupling of QCD as a function of the momentum scale $Q$. The figure is taken from $[12] \ldots \ldots \ldots \ldots \ldots$

1.2 Sketch of the lattice discritization and its main ingredients: $\psi(n)$ is the fermion field defined on the sites, $U_{\mu}(n)$ is the link variable, and the plaque-

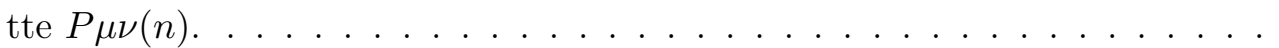

1.3 The dimensionless thermodynamic observables as a function of temperature for $N_{f}=2+1$. The gray points are from the HotQCD collaboration [21], while the colored ones are from the WB collaboration [22]. The figure is taken from $[16] \ldots \ldots \ldots \ldots \ldots$. . . . . . . . . . . . . 8

1.4 Theoretical sketch of the order of the thermal QCD transition as functions of a light quark mass $m_{u, d}$, and a heavier strange quark mass, $m_{s}$, at vanishing baryon chemical potential. Every point of the plot corresponds to a phase boundary, with an implicitly associated (pseudo-)critical temperature $T_{c}\left(m_{u, d}, m_{s}\right)$. Two scenarios are considered for $N_{f}=2, m_{u, d}=0$ : first-order (left) and second-order (right). The figure is adapted from [26] . . . . . . . .

1.5 Schematic phase diagram of QCD in the temperature $T$ and baryon chemical potential $\mu_{B}$ plane. Horizontal lines on the top panel represent the intervals of $\mu_{B}$ covered in correspondent experimental facilities. The figure is based on $[30]$.

1.6 Qualitative spacetime evolution of a relativistic heavy-ion collision. The nuclei (blue discs) propagate along the z-axis at the speed of light and collide at $z=t=0$. The QGP(orange region) medium forms at proper time $\tau \sim 1$ $\mathrm{fm} / \mathrm{c}$ and converts to a hadron resonance gas (blue region) around $\tau \sim 10$ $\mathrm{fm} / \mathrm{c}$. The figure is based on $[35] \ldots \ldots \ldots \ldots \ldots$. . . . . . . . . .

2.1 DQPM effective running coupling $\alpha_{s}=g^{2} /(4 \pi)$ as a function of the scaled temperature $T / T_{c}\left(\mu_{\mathrm{B}}\right)$ at vanishing chemical potential $\mu_{\mathrm{q}}=\mu_{\mathrm{B}}=0$ (left) and for moderate baryon chemical potentials $\mu_{\mathrm{B}} \leq 0.5 \mathrm{GeV}$ obtained within the 'scaling hypothesis' (2.1) (right) [1,57]. The lattice results for quenched QCD, $N_{f}=0$, (blue circles) are taken from Ref. [58] and for $N_{f}=2$ (black triangles) are taken from Ref. [59]. This figure is adopted from [6].

2.2 Ratios of the DQPM pole masses for light quarks (solid lines) and gluons (dashed lines) to temperature, $m_{i} / T$ (left), and ratios of the widths $\gamma_{i} / T$ (right), to temperature as a function of the scaled temperature $T / T_{c}\left(\mu_{B}\right)$ for fixed $\mu_{B}=0,0.3,0.5 \mathrm{GeV} \ldots \ldots \ldots \ldots \ldots$

2.3 Gluon (left panel) and quark (right panel) pole-masses $m_{i}$ (upper row) and their widths $\gamma_{i}$ (lower row) from the DQPM as a function of the scaled temperature $T / T_{c}\left(\mu_{\mathrm{B}}\right)$ and baryon chemical potential $\mu_{\mathrm{B}}[4] \ldots \ldots 20$ 
2.4 DQPM thermodynamic observables (solid lines): scaled entropy density $s / T^{3}$ (top left), pressure $p / T^{4}$ (top right), energy density $\epsilon / T^{4}$ (bottom left) and interaction measure $I / T^{4}$ (bottom right) as a function of temperature $T$ and at fixed baryon chemical potential $\mu_{B}$ ranging between 0 and $0.5 \mathrm{GeV}$. The $1 \mathrm{QCD}$ results obtained by the BMW collaboration are taken from Refs. $[22,60]$ (circles) for $\mu_{B}=0,0.2,0.3,0.4 \mathrm{GeV} \ldots \ldots \ldots 2$

2.5 speed of sound squared $c_{s}^{2}$ (left) and scaled specific heat $C_{V} / T^{3}$ (right) from the DQPM as a function of the scaled temperature $T / T_{c}\left(\mu_{\mathrm{B}}\right)$ and baryon chemical potential $\mu_{\mathrm{B}}$. At vanishing chemical potential we compare to IQCD results obtained by the Wuppertal-Budapest collaboration [22] (black spheres) and the HotQCD collaboration [72] (blue spheres). . . . . . 2

2.6 DQPM total elastic cross sections between different partons for the on-shell case from Eq. ( 43) evaluated in the center of mass of the collision system as a function of the collision energy $\sqrt{s}$ for $\mu_{B}=0, T=0.19 \mathrm{GeV}$ (top left), $\mu_{B}=0, T=0.19,0.316,0.458 \mathrm{GeV}$ (top right), $\mu_{B}=0,0.2,0.5 \mathrm{GeV}$ (bottom left) and for various values of baryon chemical potential $\mu_{\mathrm{B}}=0$, $0.2,0.5 \mathrm{GeV}$ for $T=2 T c\left(\mu_{\mathrm{B}}\right)$ (bottom right). The initial masses of the colliding partons are taken as the pole masses. The figure is taken from [6].

2.7 Quark off-shell interaction rate (left) as a function of scaled temperature $T / T_{c}$ for $\mu_{B}=0$ and (right) for moderate chemical potentials $\mu_{B} \leq 0.5$ $\mathrm{GeV}$ as a function of the scaled temperature $T / T_{c}\left(\mu_{B}\right)$ and the baryon chemical potential $\mu_{B}$. The contributions from the scattering with light quarks (green), antiquarks (blue), and gluons (pink) are given by the lower hatched bands, which arise from the finite statistics in the evaluation of the integrals by Monte Carlo. . . . . . . . . . . . . . . . . . . . 29

2.8 Relaxation time of a quark (left) and gluon (right) as a function of the scaled temperature $T / T_{c}\left(\mu_{B}\right)$ and the baryon chemical potential $\mu_{B}$ evaluated by the average parton interaction rate from Eq. (62). . . . . . . . . . . 31

2.9 Ratio of shear $\eta / T^{3}$ and bulk $\zeta / T^{3}$ viscosities to the temperature cubed as a function of temperature $T$ for $\mu_{B}=0$. The solid red line and the dashed blue line show the DQPM results for the shear and bulk ratios accordingly, using the parton interaction rate $\Gamma_{i}(\mathbf{p}, T, \mu)$ for the relaxation time. $\ldots 32$ 
2.10 Specific shear viscosity $\eta / s$ (left) as a function of the scaled temperature $T / T_{c}$ for $\mu_{B}=0$ and (right) for moderate chemical potentials $\mu_{B} \leq 0.5 \mathrm{GeV}$ as a function of the scaled temperature $T / T_{c}\left(\mu_{B}\right)$ and the baryon chemical potential $\mu_{B}$. The lines shows the DQPM result for $\eta_{i} / s_{\text {tot }}$ from Eq. (63) using the interaction rate $\Gamma_{i}(\mathbf{p}, T, \mu)$ for different quasiparticle species: light quarks and anti-quarks (short dashed orange line), strange quarks and antiquarks (dot-dashed magenta line), gluons (dotted blue line). The solid red line and the dashed green line show the DQPM results for total ratios of viscosity to entropy density using the parton interaction rate $\Gamma_{i}(\mathbf{p}, T, \mu)$ and the spectral width $2 \gamma_{i}(T, \mu)$ for the relaxation time. The pentagons show the $1 \mathrm{QCD}$ prediction for pure $\mathrm{SU}(3)$ gauge theory taken from Ref. [85].

2.11 Specific bulk viscosity to entropy density $\zeta / s$ (left) as a function of the scaled temperature $T / T_{C}$ for $\mu_{B}=0$ and (right) for non-zero $\mu_{B}$ as a function of the scaled temperature $T / T_{c}\left(\mu_{B}\right)$ and the baryon chemical potential $\mu_{B}$. The solid red line and the dashed green line show the DQPM results within the RTA from Eq. (64) using the parton interaction rate $\Gamma_{i}(\mathbf{p}, T, \mu)$ and the spectral width $2 \gamma_{i}(T, \mu)$ for the relaxation time. The symbols display the 1QCD data for $N_{f}=0$ pure $\mathrm{SU}(3)$ gauge theory taken from Refs. [87] (pentagons) and [88] (circles). The solid blue line shows the estimate from a Bayesian analysis of experimental heavy-ion data taken from Ref. [86]. . .

2.12 Scaled electric conductivity $\sigma_{Q} / T$ (left) as a function of the scaled temperature $T / T_{c}$ for $\mu_{B}=0$ and (right) for non-zero $\mu_{B}$ as a function of the scaled temperature $T / T_{c}\left(\mu_{B}\right)$ and the baryon chemical potential $\mu_{B}$. The solid red line and the dashed green line show the DQPM results within the RTA from Eq. (65) using the parton interaction rate $\Gamma_{i}(\mathbf{p}, T, \mu)$ and the spectral width $2 \gamma_{i}(T, \mu)$ for the relaxation time. The symbols display lQCD data for $N_{f}=2$ taken from Refs. [94,95] (red circles with brown borders), (yellow circles with green borders) and for $N_{f}=2+1$ taken from Refs. [83, 96] (spheres). The dot-dashed magenta line corresponds to the results from the first order Chapman-Enskog approximation taken from Ref. [52]. . . . . . . 37

2.13 Ratio of the baryon diffusion coefficent to the temperature squared $\kappa_{B} / T^{2} \equiv$ $\sigma_{B} / T$ (left) as a function of scaled temperature for a fixed baryon chemical potential $\mu_{B}=0$ and $0.3 \mathrm{GeV}$ and (right) as a function of scaled temperature for moderate baryon chemical potentials $\mu_{B} \leq 0.5 \mathrm{GeV}$. The dashed lines represent the AdS/CFT results for $\kappa_{B}^{S S}$, which are obtained using the results from Ref. [101] and the DQPM EoS. The dot-dashed lines correspond to the results from the first order Chapman-Enskog approximation taken from Ref. [52]. The light green dotted line and dashed black line correspond to the results from the Einstein-Maxwell-Dilaton (EMD) holographic model [49]. 39 
2.14 Left: Scaled electric conductivity, $\sigma_{\mathrm{QQ}} / T=\kappa_{\mathrm{QQ}} / T^{2}$, as a function of the scaled temperature $T / T_{c}$ at vanishing chemical potentials, $\mu_{q}=0$, from various approaches. The results from the CE (DQPM) are shown by the red solid line, and from DQPM RTA by the black dashed line. These are compared to results from the lattice QCD calculations: quenched: orange circle-shaped points [109], light green rhombus-shaped points [110], $N_{f}=2$ : light cyan circle-shaped points [94], magenta rhombus-shaped points [95], and $N_{f}=2+1$ : dark cyan circle-shaped points [96] and blue stars [111]), the kinetic partonic cascade model BAMPS (dark-green solid line with triangular-shaped points) [112], and from non-conformal EMT holographic models A [113] (violet dashed-dotted line) and B [49] (blue dotted line). For $T<T_{c}=0.158 \mathrm{GeV}$ we show results from a variety of hadronic models: SMASH $[40,105,114]$ (grey short-dashed line with squared points), effective field theory (EFT) [115] (blue dashed-dotted line), and CE tuned to a hadron gas [CE (HRG)] from Refs. [52,104, 106] (dark-red dashed line). Right: Scaled electric conductivity of the QGP at fixed scaled temperature, $T=2 T_{c}\left(\mu_{\mathrm{B}}\right)$, and $\mu_{\mathrm{Q}}=\mu_{\mathrm{S}}=0$ are shown for varying baryon chemical potential $\mu_{\mathrm{B}}$ from the DQPM RTA (black dashed line with cross-shaped points) and the CE (DQPM) (red solid line with circle-shaped points) evaluation. The figure is adopted from $[6] \ldots \ldots \ldots \ldots$

2.15 Scaled diffusion coefficients $\kappa_{q q^{\prime}} / T^{2}$ from the DQPM as a function of $T$ and $\mu_{B}$ obtained within the RTA method from Eq. (180) for moderate chemical potentials $\mu_{B} \leq 0.5 \mathrm{GeV} \ldots \ldots \ldots \ldots \ldots \ldots$

2.16 Scaled cross-electric conductivities, $\sigma_{\mathrm{QB}} / T^{2}$ (left) and $\sigma_{\mathrm{QS}} / T^{2}$ (right) as a function of scaled temperature $T / T_{c}$ at vanishing chemical potentials, $\mu_{q}=$ 0. We compare results from SMASH $[40,105]$ (grey short-dashed line with square-shaped points), the DQPM RTA (black dashed line with cross-shaped points), and the CE (DQPM) (red solid line with circle-shaped points) and CE (HRG) [52,104] (dark-red dashed line). The figure is taken from [6]. . .

2.17 Scaled cross-electric conductivities, $\sigma_{\mathrm{QB}} / T^{2}$ (left) and $\sigma_{\mathrm{QS}} / T^{2}$ (right), from the DQPM RTA (black dashed line with cross-shaped points) and the CE (DQPM) evaluation at fixed scaled temperature, $T=2 T_{c}\left(\mu_{\mathrm{B}}\right)$, shown versus the baryon chemical potential $\mu_{\mathrm{B}}$ in the range 0 to $0.5 \mathrm{GeV}$. The other chemical potentials are fixed to zero, $\mu_{\mathrm{Q}}=0$ and $\mu_{\mathrm{S}}=0$. The figure is taken from $[6] . \ldots \ldots \ldots \ldots \ldots \ldots \ldots$

2.18 Scaled strange and strange-baryon diffusion coefficients, $\kappa_{\mathrm{SS}} / T^{2}$ (left) and $\kappa_{\mathrm{SB}} / T^{2}$ (right), as a function of scaled temperature $T / T_{c}$ at vanishing chemical potentials, $\mu_{q}=0$. We compare results from CE (DQPM) (red solid line with circles), DQPM RTA (black dashed-line with crossed-shaped points), the CE (HRG) [52,104] (dark-red dashed line) and from conformal holography [117] (blue dotted line). The figure is taken from [6]. . . . . . . . . 46 
2.19 Scaled strange and strange-baryon diffusion coefficients, $\kappa_{\mathrm{SS}} / T^{2}$ (left) and $\kappa_{\mathrm{SB}} / T^{2}$ (right), from the DQPM RTA (black dashed line with cross-shaped points) and the CE (DQPM) evaluation at fixed scaled temperature, $T=$ $2 T_{c}\left(\mu_{\mathrm{B}}\right)$, shown over baryon chemical potential $\mu_{\mathrm{B}}$ in range 0 to $0.5 \mathrm{GeV}$. Further, the other chemical potentials are fixed to zero, $\mu_{\mathrm{Q}}=0$ and $\mu_{\mathrm{S}}=0$. The figure is taken from [6].

2.20 Scaled baryon diffusion coefficient, $\kappa_{\mathrm{BB}} / T^{2}$, (left) at vanishing chemical potentials, $\mu_{q}=\mu_{\mathrm{B}}=0$, as a function of the scaled temperature $T / T_{c}$ from various approaches and (right) at fixed scaled temperature, $T=2 T_{c}\left(\mu_{\mathrm{B}}\right)$, as a function of baryon chemical potential $\mu_{\mathrm{B}}$. The strange and electric potential are fixed to zero: $\mu_{\mathrm{S}}=0$ and $\mu_{\mathrm{Q}}=0$. We show results from the CE evaluation tuned to DQPM (red solid line), as described above, and tuned to a hadron gas from Refs. [52,104] (dark-red dashed line). We again compare to the calculation from DQPM RTA [2] (black dashed line with crosses) and to the non-conformal holographic model results [117] (blue dotted line) as for the electric conductivity. The figure is taken from $[6] \ldots$. . . . . . . .

3.1 Closed time contour $C$ in the Schwinger-Keldysh formalism. $C^{ \pm}$denote the time-ordered branch and the anti-time-ordered branch, respectively. . . . . . 51

3.2 Illustration of the time evolution of central $\mathrm{Au}+\mathrm{Au}$ collisions (upper row, section view) at a collisional energy of $\sqrt{s_{N N}}=19.6 \mathrm{GeV}$ within the PHSD 5.0 [143]. The local temperature $T$ (middle row), baryon chemical potential $\mu_{B}$ (lower row), as extracted from the PHSD for $y \approx 0$. The black lines (middle row) indicate the critical temperature $T_{c}=0.158 \mathrm{GeV}$. The figure

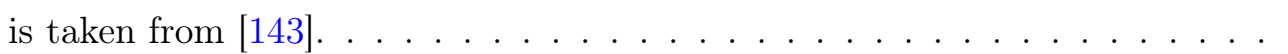

3.3 The $T$-profile (left) and $\mu_{B}$-profile (right) in $(x, y)$ at midrapidity $\left(\left|y_{\text {cell }}\right|<\right.$ 1) at 1 and $4 \mathrm{fm} / \mathrm{c}$ (from 0.5 to $6 \mathrm{fm} / \mathrm{c}$ for the $\mu_{B}$-profile) after the initial collision for $5 \%$ central $\mathrm{Pb}+\mathrm{Pb}$ collision at $158 \mathrm{~A} \mathrm{GeV}$ from PHSD5.0. The figure is taken from $[1] . \ldots \ldots \ldots \ldots$

3.4 Distributions in $T$ and $\mu_{B}$ as extracted from the DQPM equation of state in a PHSD simulation of a central $\mathrm{Pb}+\mathrm{Pb}$ collision at $158 \mathrm{~A} \mathrm{GeV}$ for cells with a temperature $T>T_{c}\left(\mu_{B}\right)$ at midrapidity $\left(\left|y_{\text {cell }}\right|<1\right)$. The scale corresponds to the number of cells in the PHSD event in the considered bin in $T-\mu_{B}$ divided by the total number of cells in the corresponding time window (see legend). The solid black line is the DQPM phase boundary for orientation; the gray dashed lines indicate ratios of $\mu_{B} / T$ ranging from 1 to 5 while the vertical line corresponds to $\mu_{B}=0$. The figure is adopted from [1]. 63

3.5 The QGP energy fraction from PHSD as a function of time $t$ in central (impact parameter $\mathrm{b}=2 \mathrm{fm}) \mathrm{Au}+\mathrm{Au}$ collisions for different collisional energies $\sqrt{s_{N N}}$ for $|y|<0.5$. The figure is taken from [7]. . . . . . . . . 64 
3.6 The rapidity distributions for $5 \%$ central $\mathrm{Au}+\mathrm{Au}$ collisions at $10.7 \mathrm{~A} \mathrm{GeV}$ for PHSD4.0 (green dot-dashed lines), PHSD5.0 with partonic cross sections and parton masses calculated for $\mu_{B}=0$ (blue dashed lines) and with cross sections and parton masses evaluated at the actual chemical potential $\mu_{B}$ in each individual space-time cell (red lines) in comparison to the experimental data from the E866 [146], E877 [147], E891 [148], E877 [149] and E896 [150] collaborations. All PHSD results are the same within the linewidth. The figure is taken from $[1] \ldots \ldots \ldots \ldots \ldots \ldots$

3.7 The rapidity distributions for $5 \%$ central $\mathrm{Pb}+\mathrm{Pb}$ collisions at $30 \mathrm{~A} \mathrm{GeV}$ for PHSD4.0 (green dot-dashed lines), PHSD5.0 with partonic cross sections and parton masses calculated for $\mu_{B}=0$ (blue dashed lines) and with cross sections and parton masses evaluated at the actual chemical potential $\mu_{B}$ in each individual space-time cell (solid red lines) in comparison to the experimental data from the NA49 Collaboration [151-153]. All PHSD results are practically the same within the linewidth. The figure is taken from [1]. .

3.8 The rapidity distributions for $5 \%$ central $\mathrm{Pb}+\mathrm{Pb}$ collisions at $158 \mathrm{~A} \mathrm{GeV}$ for PHSD4.0 (green dot-dashed lines), PHSD5.0 with partonic cross sections and parton masses calculated for $\mu_{B}=0$ (blue dashed lines) and with cross sections and parton masses evaluated at the actual chemical potential $\mu_{B}$ in each individual space-time cell (red lines) in comparison to the experimental data from the NA49 Collaboration [154-157]. All PHSD results are the same within the linewidth except for the antibaryons. The figure is taken from [1]. 66

3.9 The transverse momentum distributions for $5 \%$ central $\mathrm{Au}+\mathrm{Au}$ collisions at $11 \mathrm{~A} \mathrm{GeV}$ (left panel) and $\mathrm{Pb}+\mathrm{Pb}$ collisions at $30 \mathrm{~A} \mathrm{GeV}$ (middle panel) and $158 \mathrm{~A} \mathrm{GeV}$ (right panel), in midrapidity $(|y|<0.5)$ for PHSD4.0 (green lines), PHSD5.0 with partonic cross sections and parton masses calculated for $\mu_{B}=0$ (blue lines) and with cross sections and parton masses evaluated at the actual chemical potential $\mu_{B}$ in each individual space-time cell (red lines) in comparison to the experimental data from the E917 and E866 collaborations [158,159] (left), from the NA49 Collaboration [151-153] (middle) and $[155,156,160]$ (right). The figure is taken from $[1] \ldots \ldots$. . . . . . .

3.10 Transverse momentum distributions for 10-20\% central $\mathrm{Au}+\mathrm{Au}$ collisions at $\sqrt{s_{N N}}=19 \mathrm{GeV}$ and midrapidity $(|\mathrm{y}|<0.5)$ for PHSD4.0 (green lines), PHSD5.0 with partonic cross sections and parton masses calculated for $\mu_{B}$ $=0$ (blue dashed lines) and with cross sections and parton masses evaluated at the actual chemical potential $\mu_{B}$ in each individual space-time cell (red lines) in comparison to the experimental data from the STAR collaboration

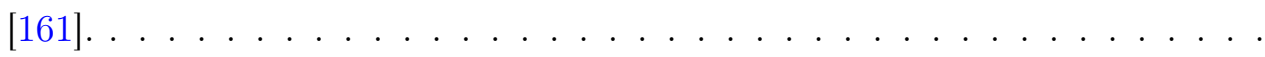


3.11 The rapidity distributions (left) and transverse momentum distributions (right) for $5 \%$ central $\mathrm{Au}+\mathrm{Au}$ collisions at $\sqrt{s_{N N}}=200 \mathrm{GeV}$ for PHSD4.0 (green dot-dashed lines), PHSD5.0 with partonic cross sections and parton masses calculated for $\mu_{B}=0$ (blue dashed lines) and with cross sections and parton masses evaluated at the actual chemical potential $\mu_{B}$ in each individual space-time cell (red lines) in comparison to the experimental data from the BRAHMS [164,165], PHENIX [162] and STAR [163] collaborations. The figure is taken from $[1] \ldots \ldots \ldots \ldots \ldots \ldots$

3.12 (Left) A non-central collision of two nuclei with impact factor $b$ leads to an almond-shaped interaction volume. (Right) The initial spatial anisotropy with respect to the reaction plane evolves under pressure gradients into a momentum anisotropy of the produced particles. . . . . . . . . . . . .

3.13 Directed flow of identified hadrons as a function of rapidity at $\sqrt{s_{N N}}=$ $200 \mathrm{GeV}$ for PHSD4.0 (green lines), PHSD5.0 with partonic cross sections and parton masses calculated for $\mu_{B}=0$ (blue dashed lines) and with cross sections and parton masses evaluated at the actual chemical potential $\mu_{B}$ in each individual space-time cell (solid red lines) in comparison to the experimental data of the STAR Collaboration [169]. . . . . . . . . . .

3.14 Directed flow of identified hadrons as a function of rapidity at $\sqrt{s_{N N}}=$ $27 \mathrm{GeV}$ for PHSD4.0 (green lines), PHSD5.0 with partonic cross sections and parton masses calculated for $\mu_{B}=0$ (blue dashed lines) and with cross sections and parton masses evaluated at the actual chemical potential $\mu_{B}$ in each individual space-time cell (red lines) in comparison to the experimental data of the STAR Collaboration [169]. . . . . . . . . . . . .

3.15 Left (a): elliptic flow of charged hadrons as a function of pseudo-rapidity $\eta$ for minimum bias $\mathrm{Au}+\mathrm{Au}$ collisions at $\sqrt{s_{N N}}=200 \mathrm{GeV}$ for PHSD4.0 (solid green line), PHSD5.0 with partonic cross sections and parton masses calculated for $\mu_{B}=0$ (blue dashed line), and with the actual $\mu_{B}$ (solid red line) in comparison to the experimental data from STAR [172] (red stars) and PHOBOS [173] (spheres) Collaborations. Middle (b): individual contributions to $v_{2}$ without their relative weights to the total $v_{2}$, which are indicated by a green solid line for PHSD5.0 with $\mu_{B}$ : the magenta dotted line corresponds to the final hadrons coming from the QGP without rescattering in the hadronic phase, the orange dashed line indicates the $v_{2}$ of hadrons coming from strings while the brown dot-dashed line shows the $v_{2}$ of hadrons coming from mesonic and baryonic resonance decays. Right (c): individual contributions to $v_{2}$ including their relative weights to the total $v_{2}$. . . . . 72

3.16 Elliptic flow of charged hadrons as a function of $p_{T}$ for $0-50 \%$ central $\mathrm{Au}+\mathrm{Au}$ collisions at $\sqrt{s_{N N}}=200 \mathrm{GeV}$ in comparison to the experimental data from the STAR Collaboration [172] (spheres). The line description is similar to

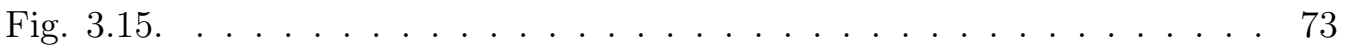


3.17 Elliptic flow of identified hadrons $\left(K^{ \pm}, p, \bar{p}, \Lambda+\Sigma^{0}, \bar{\Lambda}+\bar{\Sigma}^{0}\right)$ as a function of $p_{T}$ at $\sqrt{s_{N N}}=27 \mathrm{GeV}$ for PHSD4.0 (green lines), PHSD5.0 with partonic cross sections and parton masses calculated for $\mu_{B}=0$ (blue dashed lines) and with cross sections and parton masses evaluated at the actual chemical potential $\mu_{B}$ in each individual space-time cell (red lines) in comparison to the experimental data of the STAR Collaboration [174]. . . . . . . . . . .

4.1 The effective masses of light (left) and strange (right) quarks as a function of temperature $T$ and quark chemical potential $\mu_{q}$ from the PNJL model. The figure is adopted from [5].

4.2 Meson masses (pion, eta, kaon) as well as double quark masses $2 m_{q}$ and $m_{s}+$ $m_{q}$ from the $N_{f}=3$ PNJL model at $\mu_{q}=0$ as a function of temperature. The dotted lines indicate the $m_{\text {pole }} \pm \Gamma$, where $\Gamma$ is the imaginary part of the complex pole of the meson propagators and $m_{\text {pole }}$ is its real part (indicated by solid lines). The figure is taken from $[5] . \ldots \ldots \ldots$. . . . . . .

4.3 Meson, gluon, and quarks contributions to the total pressure as well as the total pressure (solid black line) at $\mu_{q}=0$ as a function of the temperature. The figure is taken from [5]. . . . . . . . . . . . . .

4.4 Basic thermodynamic observables from the improved PNJL model (solid lines): scaled pressure, entropy density, energy density and interaction measure as a function of temperature $T$ at $\mu_{q}=0$ in comparison to the lattice QCD results [72] (colored bands). The figure is taken from [5]. . . . . . . .

4.5 Ratio of quark pressure to the pressure in the Stefan-Boltzmann limit as a function of $\mu_{B}=3 \mu_{q}$ for a temperature of $T=0.001 \mathrm{GeV}$. We compare the resulting ratio for the PNJL approach (blue line) to the perturbative results from Ref. [190] (orange area). The figure is taken from [5] . . . . . . . . . .

4.6 Phase diagram of QCD matter described by the $N_{f}=3$ PNJL model [175]. Scaled pion pressure $P_{\pi} / T^{4}$ in the $N_{f}=3$ PNJL model in the $\left(T, \mu_{q}\right)$

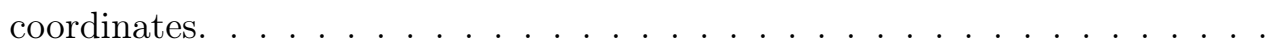

4.7 Cross section $\sigma_{u d}$ as a function of $\sqrt{s}$ at $T=190 \mathrm{MeV}$ for $\mu_{q}=0,0.1,0.2$ $\mathrm{GeV}$ (top) and at $\mu_{q}=0$ for $T=190,220$ and $300 \mathrm{MeV}$ (bottom). The figure is taken from $[5] \ldots \ldots \ldots \ldots \ldots$

4.8 Cross sections for the $q \bar{q} \rightarrow q \bar{q}$ channels: $u \bar{u} \rightarrow u \bar{u}, u \bar{d} \rightarrow u \bar{d}, u \bar{u} \rightarrow d \bar{d}$ and $u \bar{s} \rightarrow u \bar{s}$ as a function of $\sqrt{s}$ at $T=220 \mathrm{GeV}$ and $\mu_{q}=0$ (top) and for $u \bar{d} \rightarrow u \bar{d}$ at $\mu_{q}=0$ for $T=190,220,250$ and $300 \mathrm{MeV}$ (bottom). The figure is taken from $[5] . \ldots \ldots \ldots \ldots \ldots \ldots$

4.9 Resonant $u \bar{d}$ cross section as a function of $\sqrt{s}$ at $T=220 \mathrm{MeV}$ for $\mu_{q}=0$, 200, 300 and $400 \mathrm{MeV}$ (top) and for different combinations of $T, \mu_{q}$ (bottom). The figure is taken from $[5] \ldots \ldots$. . . . . . . . . . . . . 9 90

4.10 Cross section for the $s \bar{s} \rightarrow u \bar{u}$ channel calculated by the detailed balance (solid lines, DB) for $T=150,200,250 \mathrm{MeV}$ at $\mu_{q}=0$ as compared to the direct numerical calculation (dashed lines). The figure is taken from [5]. . . 91 
4.11 Modified (by the Polyakov loop) Fermi - Dirac distribution functions (used for the PNJL) in comparison to the Fermi - Dirac distribution functions (used for the NJL model) as a function of momentum for $\mu_{q}=0$ (left) and

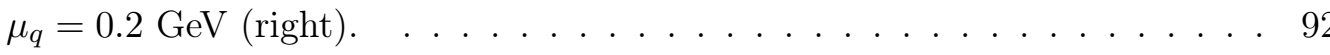

4.12 Energy averaged transition rates $\bar{w}_{i j}\left(T, \mu_{q}\right)$ for different quark-(anti-)quark scattering processes $(u \bar{d} \rightarrow u \bar{d}$ (red and orange lines), $u \bar{s} \rightarrow u \bar{s}$ (green lines), $u u \rightarrow u u$ (blue, cyan, and violet lines)) as a function of the scaled temperature $T / T_{M \pi}$ for $\mu_{q}=0$. The solid lines corresponds to the actual results from Eq. (156). Green and cyan dashed lines correspond to the results from Ref. [186], orange and violet dash-dotted lines correspond to the estimates from Ref. [194]. The figure is taken from [5] . . . . . . . . . . . . . . . . 94

4.13 Energy averaged transition rates $\bar{w}_{i j}\left(T, \mu_{q}\right)$ for different quark-quark(antiquark) scattering processes as a function of scaled temperature $T / T_{M \pi}$ for $\mu_{q}=0$ (top) and $\mu_{q}=0.2 \mathrm{GeV}$ (bottom). The solid and dashed lines correspond to the actual results from Eq. (156) for the quark-quark and the quarkantiquark scatterings. The figure is taken from [5]. . . . . . . . . . . . 95

4.14 Light and strange quarks(antiquarks) densities $n_{i}\left(T, \mu_{q}\right)$ with $f_{i}^{\phi}$ - modified Fermi distributions from Eq. (150) as a function of temperature for $\mu_{q}=0$ (left) and $\mu_{q}=0.2 \mathrm{GeV}$ (right). The solid orange and red lines correspond to the light quark and antiquark densities, while the dashed blue and violet lines correspond to the strange quark and antiquark densities. . . . . . . . 9 96

4.15 'Weighted' thermal averaged total PNJL cross-sections $\bar{\sigma}_{i j}\left(T, \mu_{q}\right)$ from Eq. (159) as a function of the scaled temperature $T / T_{M \pi}$ for $\mu_{q}=0$ (top) and $\mu_{q}=0.2$ $\mathrm{GeV}$ (bottom). The figure is taken from [5]. . . . . . . . . . . . 97

4.16 Quark relaxation times as a function of the scaled temperature $T / T_{M \pi}\left(\mu_{q}\right)$ from the PNJL model for $\mu_{q}=0$ (left) and $\mu_{q} \geq 0$ (right). The solid and the dashed lines show the results for the PNJL model using the averaged transition rates $\bar{w}_{i j}$ from Eq. (157) and the 'weighted' thermal averaged cross sections $\bar{\sigma}_{i j}$ from Eq. (158). The figure is adopted from [5]. . . . . . . 98 
4.17 Specific shear viscosity $\eta / s$ as a function of the scaled temperature $T / T_{c}$ for $\mu_{q}=0$. The solid and the dashed red lines show the results of $\eta / s$ for the PNJL model using the averaged transition rates $\bar{w}_{i j}$ from Eq. (157) and the averaged cross sections $\bar{\sigma}_{i j}$ from Eq. (158) for the evaluation of the relaxation time. We compare to the results from various models: URQMD [197] (solid green line with circles), PHSD [84] (solid blue line with triangles), SMASH [198] (solid grey line with squares), the $N_{f}=2$ linear sigma model [199] (dashed-dotted purple line), the $N_{f}=3$ NJL model [81] (dashed magenta line), DQPM [2] (dotted green line). The dashed gray line demonstrates the Kovtun-Son-Starinets bound [99] $(\eta / s)_{\mathrm{KSS}}=1 /(4 \pi)$. The symbols show lQCD data for pure SU(3) gauge theory taken from Refs. [200] (squares and rhombus), [201] (circle), [85] (pentagons). The solid blue line shows the results from a Bayesian analysis of experimental heavy-ion data [86]. The figure is taken from $[5] \ldots \ldots$. . . . . . . . . . . . . . . . . . 100

4.18 Specific shear viscosity $\eta / s$ as a function of scaled temperature $T / T_{c}$ for (left) a moderate value of the quark chemical potential $0 \leq \mu_{q} \leq 0.3$ which corresponds to a crossover phase transition and (right) whole range of the quark chemical potential $0 \leq \mu_{q} \leq 0.4 \mathrm{GeV}$. The solid (dashed) red lines show the PNJL results of $\eta / s$ for the PNJL model using the averaged transition rates $\bar{w}_{i j}(157)$ (the averaged cross sections $\bar{\sigma}_{i j}(158)$ ) for the evaluation of the relaxation time. The dotted green line and dashed purple line correspond to the results from the DQPM [2] for $\mu_{q}=0$ and $\mu_{q}=0.17 \mathrm{GeV}$. The dashed gray line demonstrates the Kovtun-Son-Starinets bound [99] $(\eta / s)_{K S S}=1 /(4 \pi)$. The figure is adopted from [5] . . . . . . . . . 101

4.19 Ratio of electric conductivity to temperature $\sigma_{Q} / T$ from Eq. (164) as a function of the scaled temperature $T / T_{c}$ for $\mu_{q}=0$. The solid (dashed) red lines show the PNJL results of $\sigma_{Q} / T$ from the PNJL model using the averaged transition rates $\bar{w}_{i j}$ from Eq. (157) (the 'weighted' thermal averaged cross sections $\bar{\sigma}_{i j}$ from Eq. (158)) for the evaluation of the relaxation time. The symbols display lQCD data for $N_{f}=2$ taken from Refs. [94,94] (red circles with brown borders), (yellow circles with green borders) and for $N_{f}=2+1$ taken from Refs. [96] (spheres) and from Ref. [111] (blue stars). We compare to predictions from the various models: the kinetic partonic cascade model BAMPS [112] (the dark-green solid line with triangles), the nonconformal holographic EMD model [49] (dashed black line), the DQPM [2] (dotted green line), and below $T_{c}=0.158 \mathrm{GeV}$ we show evaluations from hadronic models: the HRG model within the Chapman-Enskog expansion of the BE [52,104] (dashed cyan line), the $N_{f}=2$ linear sigma model [199] (dashed doted purple line), SMASH [105,114] (solid grey line with squares).

The figure is taken from $[5] . \ldots \ldots 102$ 
4.20 Ratio of the electric conductivity to the temperature $\sigma_{0} / T$ as a function of the scaled temperature $T / T_{c}$ for $\mu_{q}=0$ (left) and $\mu_{q} \geq 0$ (right). The solid (dashed) red lines show results of $\sigma_{Q} / T$ from the PNJL model using the averaged transition rates $\bar{w}_{i j}$ from Eq. (157) (the 'weighted' thermal averaged cross sections $\bar{\sigma}_{i j}$ from Eq. (158)) for the evaluation of the relaxation time. The dotted green line and dashed purple line correspond to the results from the DQPM [2] for $\mu_{q}=0$ and $\mu_{q}=0.17 \mathrm{GeV}$. The figure is adopted from [5]. 103

4.21 Ratio of specific shear viscosity $\eta / s$ to the scaled electric conductivity $\sigma_{Q} / T$ as a function of scaled temperature $T / T_{c}$ for $\mu_{q} \geq 0$. For $\mu_{q}=0$ we show the estimations from various models: the QP model [98] (dashed grey line), the DQPM [2] (dotted red line), the AdS/CFT [99,202] (dotted grey line), the $N_{f}=2$ linear sigma model [199] (dashed doted purple line). The figure is taken from [5].

5.1 Critical line of the DQPM-CP (black dashed-dotted and red solid lines) and of the DQPM (green dashed line) in the $T-\mu_{B}$-plane of the QCD phase diagram. Lines with colored areas represent lQCD estimates of $T_{c}\left(\mu_{B}\right)$ for QCD with $N_{f}=2+1$ : grey area - from Ref. [214], red area - from Ref. [192],

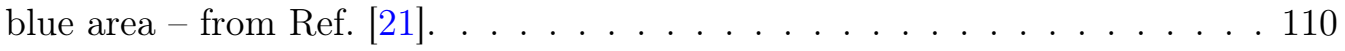

5.2 The scaled pressure $P(T) / T^{4}$ (blue line), entropy density $s(T) / T^{3}$ (red line), scaled energy density $\epsilon(T) / T^{4}$ (orange line), and interaction measure $I(T) / T^{4}$ (green line), from the DQPM-CP in comparison to the lQCD results from Refs. $[22,60]$ (circles) for zero baryon chemical potential. . . . . . . . . . . 11

5.3 The scaled pressure $p / p_{S B}$ (black line) from the DQPM-CP in comparison to the pQCD results from Ref. [190] (blue area) as a function of baryon chemical potential for fixed temperatures $T=0.05,0.10,0.150 \mathrm{GeV}$. Scenario: $\mu_{q}=$

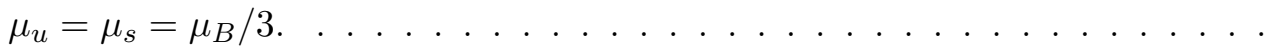

5.4 The running coupling $\alpha_{S}^{D Q P M-C P}$ from Eq. (170) as a function of the scaled temperature $T / T_{c}$ at fixed $\mu_{B}$ : for $\mu_{\mathrm{B}}=0, T_{c}=0.158 \mathrm{GeV}$ (red solid line) corresponds to the crossover phase transition and $\mu_{\mathrm{B}}=0.96, T_{c}=0.10 \mathrm{GeV}$ (green points) corresponds to the CEP. The lattice results for quenched QCD, $N_{f}=0$, (black circles) are taken from Ref. [58] and for $N_{f}=2$ (blue triangles) are taken from Ref. [59].

5.5 Effective masses of (light) quarks and gluons in the DQPM-CP along the critical line (given by Eq. (166)) as a function of the baryon chemical potential $\mu_{B}=3 \mu_{q}$. The dashed line represents the critical value of the baryon chemical potential $\mu_{C E P}=0.96 \mathrm{GeV} \ldots \ldots \ldots \ldots \ldots$

5.6 Scenario: $\mu_{q}=\mu_{u}=\mu_{s}=\mu_{B} / 3$. From top to bottom: Scaled entropy density $s / T^{3}$, pressure $p / T^{4}$ and scaled energy density $\epsilon / T^{4}$ from the DQPM (lines) as a function of temperature $T$ for various values of $\mu_{\mathrm{B}}[\mathrm{GeV}]$. The IQCD results obtained by the BMW group are taken from Refs. [22,60] (circles) and from Ref. [25] (squares). . . . . . . . . . . . 116 
5.7 Scenario: $\mu_{s}=0, \mu_{u}=\mu_{B} / 3$. From top to bottom: scaled entropy density $s / T^{3}$, pressure $p / T^{4}$ and scaled energy density $\epsilon / T^{4}$ from the DQPM-CP (lines) as a function of temperature $T$ for various values of $\mu_{\mathrm{B}}[\mathrm{GeV}]$. The lQCD results obtained by the BMW group are taken from Refs. [22,60] (circles). The PNJL results for the entropy density (colored areas) are taken from Ref. [175]. . . . . . . . . . . . . . . . . . . . 117

5.8 Scenario: $\mu_{q}=\mu_{u}=\mu_{s}=\mu_{B} / 3$. The speed of sound squared $c_{s}^{2}$ from the DQPM-CP for a crossover phase transition $\left(0 \leq \mu_{B}<0.96\right)$ as a function

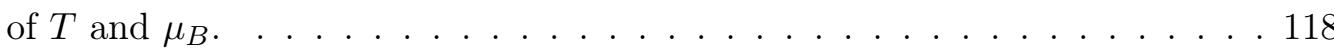

5.9 Scenario: $\mu_{q}=\mu_{u}=\mu_{s}=\mu_{B} / 3$. The speed of sound squared $c_{s}^{2}$ from the DQPM-CP for (a) $\mu_{B}=0$ and (b) $\mu_{B} \geq 0$ as a function of $T$ compared to IQCD results for $\mu_{B}=0$ obtained by the Wuppertal-Budapest collaboration [22](light red circles) and the HotQCD collaboration [72] (blue triangles).

5.10 Scenario: $\mu_{q}=\mu_{u}=\mu_{s}=\mu_{B} / 3$. The specific heat $C_{V} / T^{3}$ from the DQPM$\mathrm{CP}$ at fixed $\mu_{B}$ as a function of $T$ compared to lQCD results for $\mu_{B}=0$ of the HotQCD collaboration [72].

5.11 Trajectories of constant $s / n_{B}$ in the DQPM-CP phase diagram for $T>T_{c}$ and (a) $\mu_{q}=\mu_{u}=\mu_{s}=\mu_{B} / 3$, (b) $\mu_{s}=0, \mu_{u}=\mu_{B} / 3$ and (c) two cases in the vicinity of the critical endpoint CEP. The finite temperature crossover (black dash-dotted line) at small chemical potential switches to the large chemical potential first-order transition (red solid line) at the CEP (star), which is located at $(0.10,0.96) \mathrm{GeV} \ldots \ldots \ldots . \ldots \ldots 121$

5.12 Specific shear $\eta / s$ viscosity from the DQPM-CP (solid lines) for two setups of the strange chemical potential: (a) $\left(\mu_{s}=0, \mu_{u}=\mu_{B} / 3\right)$ and (b) $\left(\mu_{s}=\mu_{u}=\right.$ $\left.\mu_{B} / 3\right)$ as a function of the scaled temperature $T / T_{c}$ for various $\mu_{B} \geq 0$. We compare to the RTA estimates from the $N_{f}=3$ PNJL model (dashed lines) [5]. The grey dashed-dotted line demonstrates the Kovtun-Son-Starinets

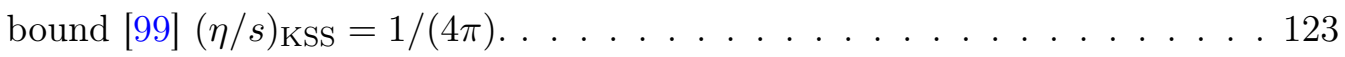

5.13 Specific bulk $\zeta / s$ viscosity from the DQPM-CP (solid lines) for two setups of the strange chemical potential: (a) $\left(\mu_{s}=0, \mu_{u}=\mu_{B} / 3\right)$ and (b) $\left(\mu_{s}=\right.$ $\left.\mu_{u}=\mu_{B} / 3\right)$ as a function of the scaled temperature $T / T_{c}$ for various $\mu_{B} \geq 0.123$

5.14 Scaled electric conductivity $\sigma_{Q Q} / T$ as a function of the scaled temperature $T / T_{c}$ from the DQPM-CP (solid lines) for two setups of the strange chemical potential: (a) $\left(\mu_{s}=0, \mu_{u}=\mu_{B} / 3\right)$ and (b) $\left(\mu_{s}=\mu_{u}=\mu_{B} / 3\right)$ as a function of the scaled temperature $T / T_{c}$ for various $\mu_{B} \geq 0$. For $\left(\mu_{s}=0, \mu_{u}=\mu_{B} / 3\right)$ we compare $\sigma_{Q Q} / T$ from DQPM-CP to the RTA estimates from the $N_{f}=3$ PNJL model (dashed lines) [5]. . . . . . . . . . . . . . . . . . . 125

5.15 Scaled baryon conductivity $\sigma_{B B} / T$ as a function of the scaled temperature $T / T_{c}$ from the DQPM-CP (solid lines) for two setups of the strange chemical potential: (a) $\left(\mu_{s}=0, \mu_{u}=\mu_{B} / 3\right)$ and (b) $\left(\mu_{s}=\mu_{u}=\mu_{B} / 3\right)$ as a function of the scaled temperature $T / T_{c}$ for various $\mu_{B} \geq 0 \ldots \ldots 126$ 
5.16 Scaled strange conductivity $\sigma_{S S} / T$ as a function of the scaled temperature $T / T_{c}$ from the DQPM-CP (solid lines) for two setups of the strange chemical potential: (a) $\left(\mu_{s}=0, \mu_{u}=\mu_{B} / 3\right)$ and (b) $\left(\mu_{s}=\mu_{u}=\mu_{B} / 3\right)$ as a function of the scaled temperature $T / T_{c}$ for various $\mu_{B} \geq 0 \ldots \ldots 127$

A.1 The scaled baryon diffusion coefficient, $\kappa_{\mathrm{BB}} / T^{2}$ (left), and the scaled electric diffusion coefficient, $\kappa_{\mathrm{QQ}} / T^{2}$ (right), for a partonic system with constant cross sections, $\sigma_{\text {tot }}=10 \mathrm{mb}$ at $\mu_{B}=0$ as a function of $T$ from different approaches. We compare the DQPM RTA $\left(\tau_{i}\left(\mathbf{p}, T, \mu_{B}\right)\right)$ results from Eq. (180) (for a system obeying quantum statistics, i.e. $a_{i}= \pm 1$ in Eq. (58)) with (blue dashed line) and without (red dashed-double-dotted line) inelastic, flavor-changing channels to the CE (DQPM) results either in RTA $\left(\tau_{0}\left(T, \mu_{B}\right)\right)$ (green dashed-dotted line) from Eq. (180) (for a system obeying classical statistics, i.e. $a_{i}=0$ in Eq. (58)) or for the "full" linearized collision term (orange solid line) from Eq. (72) $\left(a_{i}=0\right.$ in Eq. (58)). . . . . 146

B.1 Feynman diagrams of quark-quark processes for the $t$ - and $u$ - channels. . . 147

B.2 Feynman diagrams of quark-antiquark processes for the $t$ - and $s-$ channels. 148

C.1 The second-order event plane resolution for $\mathrm{Au}+\mathrm{Au}$ collisions at $\sqrt{s_{N N}}=14.5$

$\mathrm{GeV}$ from PHSD5.0 as a function of centrality compared to the estimates from the STAR Collaboration [238] (red stars) reconstructed by using the TPC tracks $(|\eta|<1) \ldots \ldots \ldots \ldots \ldots 1 . \ldots \ldots \ldots$

\section{List of Tables}

1 Fundamental properties of the parton species considered in the quark-gluon plasma. Here, $e=\sqrt{4 \pi \alpha_{e m}}$ denotes the elementary electric charge in natural units, while $q_{i}$ is dimensionless electric charge of the parton specie ' ${ }^{\prime}{ }^{\prime} . \quad$. . . 31

2 Parameters of the PNJL model considered in Refs. [5, 175] . . . . . . . . . 80

3 Parameters of the effective potential $\mathrm{U}(\phi, \bar{\phi}, \mathrm{T})$ used in this work. . . . . . 80

4 Mesons which can be exchanged in the $t$ - and $u$ - channels in the different quark-quark cross sections. . . . . . . . . . . . . . . . . . 148

5 Mesons which can be exchanged in the $s-$ and $t$ - channels in the different quark-antiquark cross sections. . . . . . . . . . . . . . . . . . 149 


\section{Comparison study: constant isotropic cross sections}

In order to evaluate the systematic differences between the RTA and the CE approaches we consider here a 'model study' by assuming a total cross section of $\sigma_{\text {tot }}=10 \mathrm{mb}$ for all interactions in the DQPM. For this comparison we incorporate the same assumptions as described in Chapter 2.

In Fig. A.1 we show the results for the scaled baryon diffusion coefficient, $\kappa_{\mathrm{BB}} / T^{2}$ (left plot), and the scaled electric diffusion coefficient, $\kappa_{\mathrm{QQ}} / T^{2}=\sigma_{\mathrm{el}} / T$ (right plot) as a function of temperature $T$ at $\mu_{\mathrm{B}}=0$. The DQPM RTA calculations are presented for two cases: (i) where all binary channels, including the inelastic ones (flavor changing processes $q \bar{q} \rightarrow q^{\prime} \bar{q}^{\prime}$ ), are considered (blue dashed line); (ii) only the elastic channels are taken into account (red dashed-double-dotted line). For the DQPM RTA results presented here Eq. (180) has been used, where we employed the momentum-dependent relaxation times $\tau_{i}\left(\mathbf{p}, T, \mu_{B}\right)$ :

$$
\tau_{i}\left(\mathbf{p}, T, \mu_{B}\right)=\frac{1}{\Gamma_{i}\left(\mathbf{p}, T, \mu_{B}\right)}
$$

The CE (DQPM) calculations are presented in Fig. A.1 also for two cases with only elastic channels. In the first case we evaluate the coefficients in RTA with the help of Eq. (180) (for a system obeying classical statistics, i.e. $a_{i}=0$ in Eq. (58)) under the assumption of the simplistic momentum-independent relaxation time

$$
\tau_{0}\left(T, \mu_{B}\right)=\frac{1}{n_{\text {tot }} \sigma_{\text {tot }}} .
$$

The results are presented by the orange solid lines. For the second case (Full) we consider the full linearized Boltzmann equation via Eq. (72) (for a system obeying classical statistics, i.e. $a_{i}=0$ in Eq. (58) ). The results are presented by the green dashed-dotted line. This 'model study' shows the influence of the linearized Boltzmann equation compared to its relaxation-time approximation, and the influence of the inelastic channels compared to its neglection. We find that the consideration of the full linearized collision term effectively reduces the scattering rate of a specific particle species, while in the RTA the scattering rate is overestimated. This is because in the collision term not only the scattering of particles from a specific momentum bin into all other momentum bins is considered, but also the rescattering into this particular momentum bin is accounted for (gain and loss term). As argued in Ref. [104] such an overestimation of the scattering rate leads to a decrease of the diffusion coefficients from RTA (which are anti-proportional to the rate).

Furthermore, we find that the inelastic channels lead to a further decrease of the dif- 

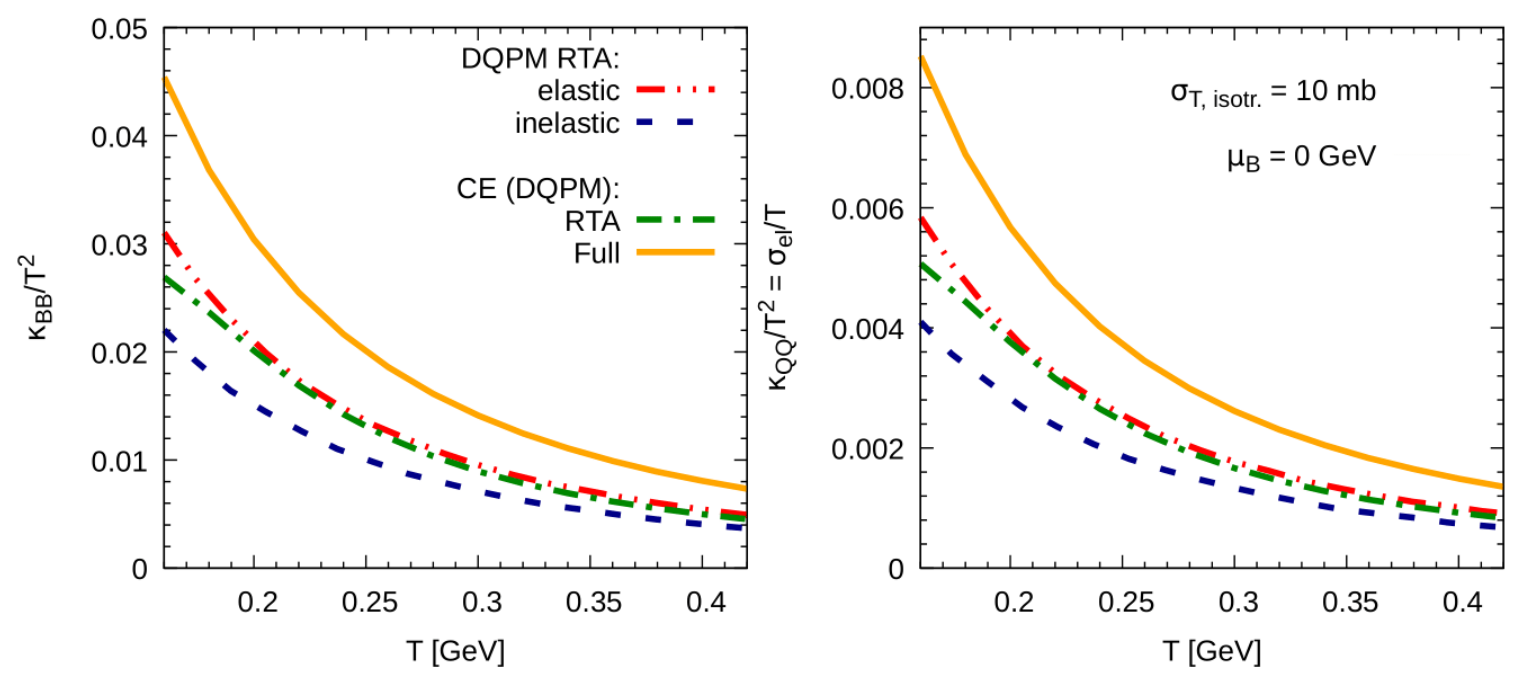

Figure A.1: The scaled baryon diffusion coefficient, $\kappa_{\mathrm{BB}} / T^{2}$ (left), and the scaled electric diffusion coefficient, $\kappa_{\mathrm{QQ}} / T^{2}$ (right), for a partonic system with constant cross sections, $\sigma_{\text {tot }}=10 \mathrm{mb}$ at $\mu_{B}=0$ as a function of $T$ from different approaches. We compare the DQPM RTA $\left(\tau_{i}\left(\mathbf{p}, T, \mu_{B}\right)\right)$ results from Eq. (180) (for a system obeying quantum statistics, i.e. $a_{i}= \pm 1$ in Eq. (58)) with (blue dashed line) and without (red dasheddouble-dotted line) inelastic, flavor-changing channels to the CE (DQPM) results either in RTA $\left(\tau_{0}\left(T, \mu_{B}\right)\right)$ (green dashed-dotted line) from Eq. (180) (for a system obeying classical statistics, i.e. $a_{i}=0$ in Eq. (58)) or for the "full" linearized collision term (orange solid line) from Eq. (72) $\left(a_{i}=0\right.$ in Eq. (58)).

fusion coefficients due to the repeated effective increase of the scattering rate as shown in Fig. A.1. Comparing the elastic version of the DQPM RTA evaluation with the CE (DQPM) calculation in the RTA limit employing $\tau_{0}\left(T, \mu_{B}\right)$, we find a good agreement of the results at high $T$. This can be explained by the fact that the only difference between both calculations - DQPM RTA and CE (DQPM) in the RTA limit - is the consideration of quantum corrections and the more sophisticated (momentum-dependent) relaxation time in DQPM RTA. 


\section{B}

\section{Quark-(anti)quark scattering in the $N_{f}=3$ PNJL model}

\section{B.0.1 Quark-quark scattering}

There are two possible Feynman diagrams for quark-quark scattering, the $t-$ and $u-$ channels as indicated in Fig. B.1.
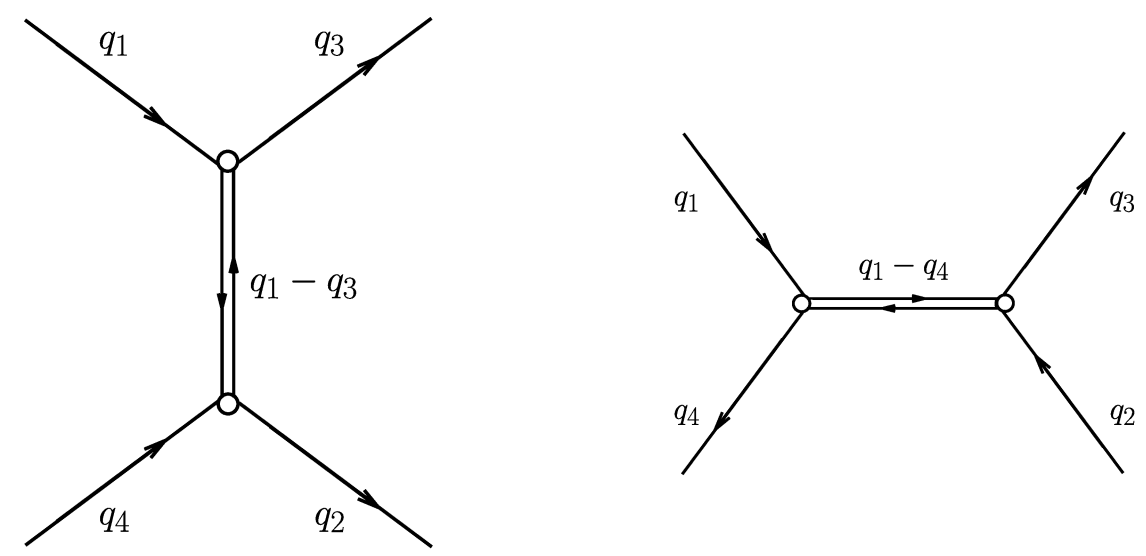

Figure B.1: Feynman diagrams of quark-quark processes for the $t$ - and $u$ - channels.

The associated squared of the matrix elements for the $t-$ and $u-$ channels and their interference term are defined as

$$
\begin{gathered}
\frac{1}{4 N_{C}^{2}} \sum_{s, c}\left|M_{u}\right|^{2}=\left|D_{u}^{S}\right|^{2} u_{14}^{+} u_{23}^{+}+\left|D_{u}^{P}\right|^{2} u_{14}^{-} u_{23}^{-}, \\
\frac{1}{4 N_{C}^{2}} \sum_{s, c}\left|M_{u t}\right|=\frac{1}{4 N_{C}}\left[D_{t}^{S} D_{u}^{S *}\left(t_{13}^{+} t_{24}^{+}-s_{12}^{+} s_{34}^{+}+u_{14}^{+} u_{23}^{+}\right)\right. \\
-D_{t}^{S} D_{u}^{P *}\left(t_{13}^{+} t_{24}^{+}-s_{12}^{-} s_{34}^{-}+u_{14}^{-} u_{23}^{-}\right) \\
-D_{t}^{P} D_{u}^{S *}\left(t_{13}^{-} t_{24}^{-}-s_{12}^{-} s_{34}^{-}+u_{14}^{+} u_{23}^{+}\right) \\
\left.+D_{t}^{P} D_{u}^{P *}\left(t_{13}^{-} t_{24}^{-}-s_{12}^{+} s_{34}^{+}+u_{14}^{-} u_{23}^{-}\right)\right] \\
\frac{1}{4 N_{C}^{2}} \sum_{s, c}\left|M_{t}\right|^{2}=\left|D_{t}^{S}\right|^{2} t_{13}^{+} t_{24}^{+}+\left|D_{t}^{P}\right|^{2} t_{13}^{-} t_{24}^{-} .
\end{gathered}
$$

Here we use the shorthand notation

$$
t_{i j}^{ \pm}=t-\left(m_{i} \pm m_{j}\right)^{2}
$$




$$
\begin{gathered}
u_{i j}^{ \pm}=u-\left(m_{i} \pm m_{j}\right)^{2}, \\
s_{i j}^{ \pm}=s-\left(m_{i} \pm m_{j}\right)^{2} .
\end{gathered}
$$

$D^{S}$ and $D^{P}$ denote the effective propagators of the exchanged scalar and pseudoscalar meson, respectively, which reads

$$
D^{a}=\frac{2 i g_{m}}{1-2 g_{m} \Pi_{f f^{\prime}}^{ \pm}\left(k_{0}, \vec{k}\right)},
$$

where $g_{m}$ is the coupling constant [186] and $\Pi_{f f^{\prime}}^{ \pm}\left(k_{0}, \vec{k}\right)$ is the quark-antiquark polarisation function (see Chapter 4 for further details on $\Pi_{f f^{\prime}}^{ \pm}$and $g_{m}$ ).

Table 4 displays the mesons which can be exchanged in the $t$ - and $u$ - channels in the different quark-quark cross sections [186].

\begin{tabular}{|c|c|c|}
\hline Process & $\begin{array}{c}\text { Exchanged mesons } \\
\text { in } u \text {-channel }\end{array}$ & $\begin{array}{c}\text { Exchanged mesons } \\
\text { in } t \text {-channel }\end{array}$ \\
\hline$u d \rightarrow u d$ & $\pi, \sigma_{\pi}$ & $\pi, \eta, \eta^{\prime}, \sigma_{\pi}, \sigma, \sigma^{\prime}$ \\
\hline$u u \rightarrow u u$ & $\pi, \eta, \eta^{\prime}, \sigma_{\pi}, \sigma, \sigma^{\prime}$ & $\pi, \eta, \eta^{\prime}, \sigma_{\pi}, \sigma, \sigma^{\prime}$ \\
\hline$u s \rightarrow u s$ & $\mathrm{~K}, \sigma_{K}$ & $\eta, \eta^{\prime}, \sigma, \sigma^{\prime}$ \\
\hline$s s \rightarrow s s$ & $\eta, \eta^{\prime}, \sigma, \sigma^{\prime}$ & $\eta, \eta^{\prime}, \sigma, \sigma^{\prime}$ \\
\hline
\end{tabular}

Table 4: Mesons which can be exchanged in the $t$ - and $u$ - channels in the different quark-quark cross sections.

\section{B.0.2 Quark-antiquark scattering}

For quark-antiquark scattering only $s-$ and $t-$ channels are possible. The corresponding diagrams are shown in Fig. B.2.
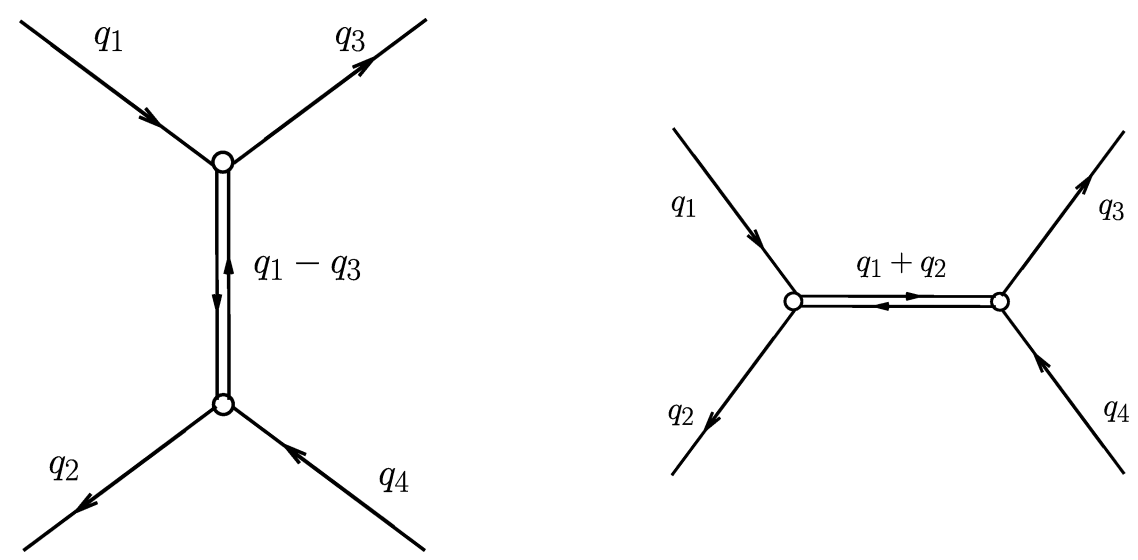

Figure B.2: Feynman diagrams of quark-antiquark processes for the $t$ - and $s$ - channels.

The corresponding matrix elements (squared) for the $s-$ and $t$ - channels and their 
interference term reads

$$
\begin{gathered}
\frac{1}{4 N_{C}^{2}} \sum_{s, c}\left|M_{s}\right|^{2}=\left|D_{s}^{S}\right|^{2} s_{12}^{+} s_{34}^{+}+\left|D_{s}^{P}\right|^{2} s_{12}^{-} s_{34}^{-}, \\
\frac{1}{4 N_{C}^{2}} \sum_{s, c}\left|M_{s t}\right|=\frac{1}{4 N_{C}}\left[D_{s}^{S} D_{t}^{S *}\left(s_{12}^{+} s_{34}^{+}-u_{14}^{+} u_{23}^{+}+t_{13}^{+} t_{24}^{+}\right)\right. \\
-D_{s}^{S} D_{t}^{P *}\left(s_{12}^{+} s_{34}^{+}-u_{14}^{-} u_{24}^{-}+t_{13}^{-} t_{24}^{-}\right) \\
-D_{s}^{P} D_{t}^{S *}\left(s_{12}^{-} s_{34}^{-}-u_{14}^{-} u_{23}^{-}+t_{13}^{+} t_{24}^{+}\right) \\
\left.+D_{s}^{P} D_{t}^{P *}\left(s_{12}^{-} s_{34}^{-}-u_{14}^{+} u_{23}^{+}+t_{13}^{-} t_{24}^{-}\right)\right], \\
\frac{1}{4 N_{C}^{2}} \sum_{s, c}\left|M_{t}\right|^{2}=\left|D_{t}^{S}\right|^{2} t_{13}^{+} t_{24}^{+}+\left|D_{t}^{P}\right|^{2} t_{13}^{-} t_{24}^{-} .
\end{gathered}
$$

Table 5 presents the mesons which can be exchanged in the $s-$ and $t$ - channels in the different quark-(anti-)quark cross sections [186].

\begin{tabular}{|c|c|c|}
\hline Process & $\begin{array}{c}\text { Exchanged mesons } \\
\text { in s-channel }\end{array}$ & $\begin{array}{c}\text { Exchanged mesons } \\
\text { in t-channel }\end{array}$ \\
\hline$u \bar{d} \rightarrow u \bar{d}$ & $\pi, \sigma_{\pi}$ & $\pi, \eta, \eta^{\prime}, \sigma_{\pi}, \sigma, \sigma^{\prime}$ \\
\hline$u \bar{u} \rightarrow u \bar{u}$ & $\pi, \eta, \eta^{\prime}, \sigma_{\pi}, \sigma, \sigma^{\prime}$ & $\pi, \eta, \eta^{\prime}, \sigma_{\pi}, \sigma, \sigma^{\prime}$ \\
\hline$u \bar{u} \rightarrow d \bar{d}$ & $\pi, \eta, \eta^{\prime}, \sigma_{\pi}, \sigma, \sigma^{\prime}$ & $\pi, \sigma_{\pi}$ \\
\hline$u \bar{s} \rightarrow u \bar{s}$ & $\mathrm{~K}, \sigma_{K}$ & $\eta, \eta^{\prime}, \sigma, \sigma^{\prime}$ \\
\hline$u \bar{u} \rightarrow s \bar{s}$ & $\eta, \eta^{\prime}, \sigma, \sigma^{\prime}$ & $\mathrm{K}, \sigma_{K}$ \\
\hline$s \bar{s} \rightarrow u \bar{u}$ & $\eta, \eta^{\prime}, \sigma, \sigma^{\prime}$ & $\mathrm{K}, \sigma_{K}$ \\
\hline$s \bar{s} \rightarrow s \bar{s}$ & $\eta, \eta^{\prime}, \sigma, \sigma^{\prime}$ & $\eta, \eta^{\prime}, \sigma, \sigma^{\prime}$ \\
\hline
\end{tabular}

Table 5: Mesons which can be exchanged in the $s-$ and $t$ - channels in the different quark-antiquark cross sections. 


\section{Application of the Event-Plane method to the PHSD}

Here we describe the basic concepts of the so-called standard event-plane method [168]. It estimates the azimuthal angle of the reaction plane employing the observed event plane angle, which is obtained from the anizotropic flow. The procedure is performed for each harmonic of the Fourier decomposition.

The event flow vector $Q_{n}$ is a two-dimensional vector in the transverse plane:

$$
\begin{aligned}
& Q_{n, x}=Q_{n} \cos \left(n \Psi_{n}\right)=\sum_{i} \omega_{i} \cos \left(n \phi_{i}\right), \\
& Q_{n, y}=Q_{n} \sin \left(n \Psi_{n}\right)=\sum_{i} \omega_{i} \sin \left(n \phi_{i}\right),
\end{aligned}
$$

where $\phi_{i}$ and $\omega_{i}$ denote the laboratory azimuthal angle and weight for particle $i$, with $\omega_{i}(-y)=-\omega_{i}(y)$ for odd harmonics. In Eq. (194) $\Psi_{n}$ denotes the event plane angle, which reads

$$
\Psi_{n}=\frac{1}{n} \arctan \left[\frac{Q_{n, x}}{Q_{n, y}}\right]
$$

The observed $v_{n}^{o b s}$ is the $n$th harmonic of the azimuthal particle distribution with respect to the event plane

$$
v_{n}^{o b s}=<\cos \left[n\left(\phi_{i}-\Psi_{n}\right)\right]>.
$$

Furthernore, the finite multiplicity limits the acuracy of the reaction plane angle. Therefore, to obtain the flow coefficient $v_{n}$, the observed value $v_{n}^{o b s}$ must be corrected for the resolution of the reaction plane a follows

$$
\begin{aligned}
& v_{n}=v_{n}^{o b s} / R_{n}, \\
& R_{n}=<\cos \left[n\left(\Psi_{n}-\Psi_{R P}\right)\right]>.
\end{aligned}
$$

The resolution correction factor $R_{n}$ depends on the multiplicity of particles that determine the flow vector and the average flow of these particles throught the resolution parameter as

$$
\begin{aligned}
& \chi=v_{n} \sqrt{M}, \\
& R_{k}(\chi)=\sqrt{\pi} / 2 \chi \exp \left(-\chi^{2} / 2\right)\left(I_{(k-1) / 2}\left(\chi^{2} / 2\right)+I_{(k+1) / 2}\left(\chi^{2} / 2\right)\right) .
\end{aligned}
$$

Here $I_{(k \pm 1) / 2}$ denotes the modified Bessel function. In order to estimate $R_{n}$ one divides the full event up into two independent sub-events of equal multiplicity. In our case we follow Refs. [238, 239], where the $\eta-s u b$ method is utilized. The particles are divided into two 
sub-events for $\eta<0$ and $\eta>0$. Moreover, an additional gap of 0.1 between the sub-events is employed, and then an average of the results from the two sub-events is performed. The resulting $R_{n}(s u b)$, i.e. the solution for $\chi$ in Eq. (200), is obtained by iteration. The full event plane resolution is obtained using Eq. (200) from the resolution of the sub-events by

$$
R_{n}^{f u l l}=R_{n}\left(\sqrt{2} \chi_{s u b}\right) .
$$

For all results presented in Chapter 3 we employ the TPC event plane method. As an example we consider a second-order event plane angle $\left(\Psi_{n}\right)$, which reconstructed for the particles at mid-rapidity $|\eta|<1$ following the method used by the STAR Collaboration [238]. The second-order event plane resolution estimated for $\mathrm{Au}+\mathrm{Au}$ collisions at $\sqrt{s_{N N}}=14.5 \mathrm{GeV}$ is displayed in Fig. C.1 as a function of centrality. The PHSD results (blue dots) are compared with the estimates from the STAR Collaboration [238] (red stars) reconstructed by using the TPC tracks $(|\eta|<1)$.

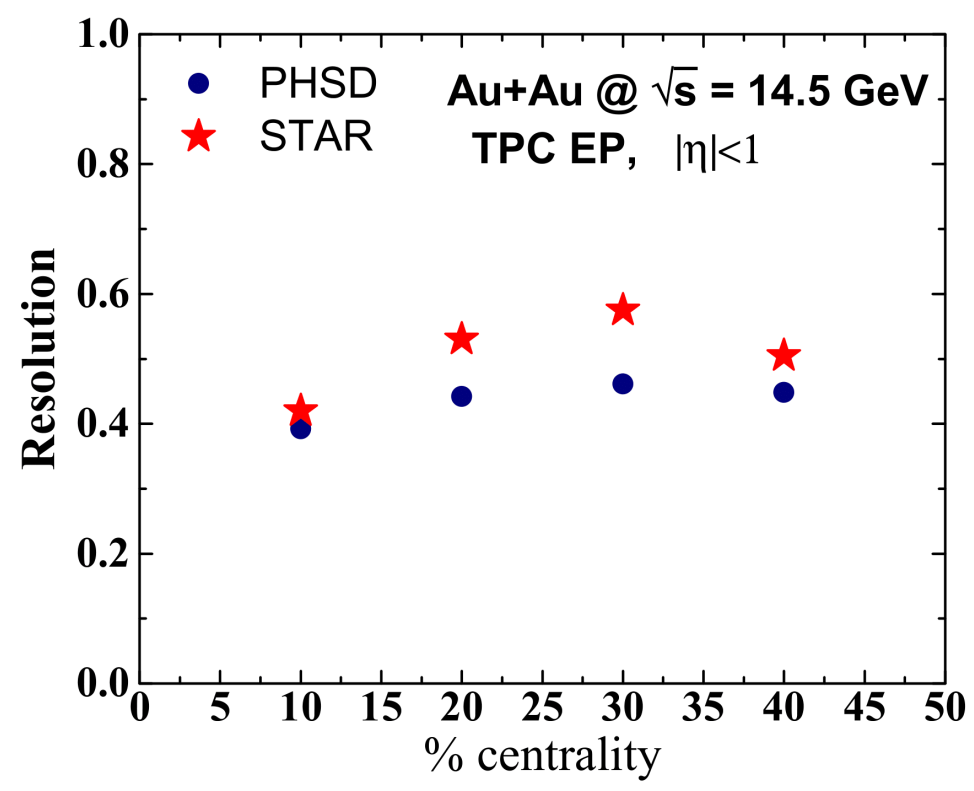

Figure C.1: The second-order event plane resolution for $\mathrm{Au}+\mathrm{Au}$ collisions at $\sqrt{s_{N N}}=14.5$ $\mathrm{GeV}$ from PHSD5.0 as a function of centrality compared to the estimates from the STAR Collaboration [238] (red stars) reconstructed by using the TPC tracks $(|\eta|<1)$. 


\section{Abbreviations}

CEP critical endpoint

DQPM dynamical quasiparticle model

EoS equation of state

FRG Functional Renormalisation Group

HICs heavy-ion collisions

HSD Hadron-String-Dynamics

LHC Large Hadron Collider

1QCD lattice QCD

PHSD Parton-Hadron-String-Dynamics

PNJL Polyakov extended Nambu-Jona Lasinio model

QCD quantum chromodynamics

QED quantum electrodynamics

QGP quark-gluon plasma

RHIC Relativistic Heavy-Ion Collider

RPA random-phase approximation

RTA relaxation-time approximation 


\section{References}

[1] Moreau, P. et al. Exploring the partonic phase at finite chemical potential within an extended off-shell transport approach. Phys. Rev. C 100, 014911 (2019).

[2] Soloveva, O., Moreau, P. and Bratkovskaya, E. Transport coefficients for the hot quark-gluon plasma at finite chemical potential $\mu_{B}$. Phys. Rev. C 101, 045203 (2020).

[3] Soloveva, O. et al. Transport coefficients of hot and dense matter. Springer Proc. Phys. 250, 447-451 (2020).

[4] Soloveva, O. et al. Exploring the partonic phase at finite chemical potential in and out-of equilibrium. Particles 3, 178-192 (2020).

[5] Soloveva, O., Fuseau, D., Aichelin, J. and Bratkovskaya, E. Shear viscosity and electric conductivity of a hot and dense QGP with a chiral phase transition. Phys. Rev. C 103, 054901 (2021).

[6] Fotakis, J. A., Soloveva, O., Greiner, C., Kaczmarek, O. and Bratkovskaya, E. Diffusion coefficient matrix of the strongly interacting quark-gluon plasma. Phys. Rev. D 104, 034014 (2021).

[7] Moreau, P. et al. Properties of the quark-gluon plasma created in heavy-ion collisions. Astronomische Nachrichten 342, 715-726 (2021).

[8] Soloveva, O., Moreau, P. and Bratkovskaya, E. QCD at finite chemical potential in and out-of equilibrium. Phys. Scripta 96, 124067 (2021).

[9] Soloveva, O., Aichelin, J. and Bratkovskaya, E. Transport properties and equationof-state of hot and dense QGP matter near the critical end-point in the phenomenological dynamical quasi-particle model. arXiv:2108.08561 (2021).

[10] Politzer, H. D. Reliable Perturbative Results for Strong Interactions? Phys. Rev. Lett. 30, 1346-1349 (1973).

[11] Gross, D. J. and Wilczek, F. Ultraviolet Behavior of Nonabelian Gauge Theories. Phys. Rev. Lett. 30, 1343-1346 (1973).

[12] Zyla, P. A. et al. Review of Particle Physics. PTEP 2020, 083C01 (2020).

[13] Bethke, S. Experimental tests of asymptotic freedom. Prog. Part. Nucl. Phys. 58, 351-386 (2007).

[14] Bornyakov, V. G. et al. Baryonic flux in quenched and two flavor dynamical QCD. Phys. Rev. D 70, 054506 (2004). 
[15] Suganuma, H., Takahashi, T. T., Okiharu, F. and Ichie, H. Study of quark confinement in Baryons with lattice QCD. Nucl. Phys. B Proc. Suppl. 141, $92-98$ (2005).

[16] Ratti, C. Lattice QCD and heavy ion collisions: a review of recent progress. Rept. Prog. Phys. 81, 084301 (2018).

[17] Braun, J. et al. Chiral susceptibility in $(2+1)$-flavor QCD. Phys. Rev. D 102, 056010 (2020).

[18] Fischer, C. S. QCD at finite temperature and chemical potential from DysonSchwinger equations. Prog. Part. Nucl. Phys. 105, 1-60 (2019).

[19] Wilson, K. G. Confinement of Quarks. Phys. Rev. D 10, 2445-2459 (1974).

[20] Montvay, I. and Munster, G. Quantum fields on a lattice. Cambridge Monographs on Mathematical Physics (Cambridge University Press, 1997).

[21] Bazavov, A. et al. Chiral crossover in QCD at zero and non-zero chemical potentials. Phys. Lett. B 795, 15-21 (2019).

[22] Borsanyi, S. et al. Full result for the QCD equation of state with $2+1$ flavors. Phys. Lett. B 730, 99-104 (2014).

[23] Gavai, R. V. and Gupta, S. Pressure and nonlinear susceptibilities in QCD at finite chemical potentials. Phys. Rev. D 68, 034506 (2003).

[24] Allton, C. R. et al. The Equation of state for two flavor QCD at nonzero chemical potential. Phys. Rev. D 68, 014507 (2003).

[25] Borsányi, S. et al. Lattice QCD equation of state at finite chemical potential from an alternative expansion scheme. Phys. Rev. Lett. 126, 232001 (2021).

[26] Cuteri, F., Philipsen, O. and Sciarra, A. On the order of the QCD chiral phase transition for different numbers of quark flavours. arXiv:2107.12739 (2021).

[27] Weiss, N. The Effective Potential for the Order Parameter of Gauge Theories at Finite Temperature. Phys. Rev. D 24, 475 (1981).

[28] Alford, M. G., Schmitt, A., Rajagopal, K. and Schäfer, T. Color superconductivity in dense quark matter. Rev. Mod. Phys. 80, 1455-1515 (2008).

[29] Shuryak, E. V. Quark-Gluon Plasma and Hadronic Production of Leptons, Photons and Psions. Phys. Lett. B 78, 150 (1978).

[30] Caines, H. The Search for Critical Behavior and Other Features of the QCD Phase Diagram - Current Status and Future Prospects. Nucl. Phys. A 967, 121-128 (2017).

[31] Aoki, Y., Endrodi, G., Fodor, Z., Katz, S. and Szabo, K. The Order of the quantum chromodynamics transition predicted by the standard model of particle physics. Nature 443, 675-678 (2006). 
[32] Chatrchyan, S. et al. Observation and studies of jet quenching in $\mathrm{PbPb}$ collisions at nucleon-nucleon center-of-mass energy $=2.76 \mathrm{TeV}$. Phys. Rev. C 84, 024906 (2011).

[33] Adam, J. et al. Enhanced production of multi-strange hadrons in high-multiplicity proton-proton collisions. Nature Phys. 13, 535-539 (2017).

[34] Chatrchyan, S. et al. Suppression of non-prompt $J / \psi$, prompt $J / \psi$, and $\mathrm{Y}(1 \mathrm{~S})$ in $\mathrm{PbPb}$ collisions at $\sqrt{s_{N N}}=2.76 \mathrm{TeV}$. JHEP 05, 063 (2012).

[35] Braun-Munzinger, P. and Dönigus, B. Loosely-bound objects produced in nuclear collisions at the LHC. Nucl. Phys. A 987, 144-201 (2019).

[36] Bass, S. A. et al. Microscopic models for ultrarelativistic heavy ion collisions. Prog. Part. Nucl. Phys. 41, 255-369 (1998).

[37] Bleicher, M. et al. Relativistic hadron hadron collisions in the ultrarelativistic quantum molecular dynamics model. J. Phys. G 25, 1859-1896 (1999).

[38] Cassing, W. and Bratkovskaya, E. Hadronic and electromagnetic probes of hot and dense nuclear matter. Phys. Rept. 308, 65-233 (1999).

[39] Sun, K.-J., Ko, C. M. and Lin, Z.-W. Light nuclei production in a multiphase transport model for relativistic heavy ion collisions. Phys. Rev. C 103, 064909 (2021).

[40] Weil, J. et al. Particle production and equilibrium properties within a new hadron transport approach for heavy-ion collisions. Phys. Rev. C 94, 054905 (2016).

[41] Karpenko, I. A., Huovinen, P., Petersen, H. and Bleicher, M. Estimation of the shear viscosity at finite net-baryon density from $A+A$ collision data at $\sqrt{s_{\mathrm{NN}}}=7.7-200$ GeV. Phys. Rev. C 91, 064901 (2015).

[42] Ryu, S. et al. Importance of the Bulk Viscosity of QCD in Ultrarelativistic Heavy-Ion Collisions. Phys. Rev. Lett. 115, 132301 (2015).

[43] Cassing, W. and Bratkovskaya, E. Parton-Hadron-String Dynamics: an off-shell transport approach for relativistic energies. Nucl. Phys. A 831, 215-242 (2009).

[44] Arnold, P. B., Moore, G. D. and Yaffe, L. G. Transport coefficients in high temperature gauge theories. 2. Beyond leading log. JHEP 05, 051 (2003).

[45] Cassing, W. Dynamical quasiparticles properties and effective interactions in the sQGP. Nucl. Phys. A 795, 70-97 (2007).

[46] Peshier, A. and Cassing, W. The Hot non-perturbative gluon plasma is an almost ideal colored liquid. Phys. Rev. Lett. 94, 172301 (2005).

[47] Berrehrah, H., Bratkovskaya, E., Steinert, T. and Cassing, W. A dynamical quasiparticle approach for the QGP bulk and transport properties. Int. J. Mod. Phys. E 25, 1642003 (2016). 
[48] Attems, M. et al. Thermodynamics, transport and relaxation in non-conformal theories. JHEP 10, 155 (2016).

[49] Rougemont, R., Critelli, R., Noronha-Hostler, J., Noronha, J. and Ratti, C. Dynamical versus equilibrium properties of the QCD phase transition: A holographic perspective. Phys. Rev. D 96, 014032 (2017).

[50] Jackson, G. and Peshier, A. Re-running the QCD shear viscosity. J. Phys. G 45, 095001 (2018).

[51] Thakur, L., Srivastava, P. K., Kadam, G. P., George, M. and Mishra, H. Shear viscosity $\eta$ to electrical conductivity $\sigma_{e l}$ ratio for an anisotropic QGP. Phys. Rev. D 95, 096009 (2017).

[52] Greif, M., Fotakis, J. A., Denicol, G. S. and Greiner, C. Diffusion of conserved charges in relativistic heavy ion collisions. Phys. Rev. Lett. 120, 242301 (2018).

[53] Mykhaylova, V., Bluhm, M., Redlich, K. and Sasaki, C. Quark-flavor dependence of the shear viscosity in a quasiparticle model. Phys. Rev. D 100, 034002 (2019).

[54] Zhao, Y.-P. Thermodynamic properties and transport coefficients of QCD matter within the nonextensive Polyakov-Nambu-Jona-Lasinio model. Phys. Rev. D 101, 096006 (2020).

[55] Abhishek, A., Das, A., Kumar, D. and Mishra, H. Thermoelectric transport coefficients of quark matter (2020).

[56] Cassing, W. QCD thermodynamics and confinement from a dynamical quasiparticle point of view. Nucl. Phys. A 791, 365-381 (2007).

[57] Soloveva, O. et al. Exploring the partonic phase at finite chemical potential in and out-of equilibrium. J. Phys. Conf. Ser. 1602, 012012 (2020).

[58] Kaczmarek, O., Karsch, F., Zantow, F. and Petreczky, P. Static quark anti-quark free energy and the running coupling at finite temperature. Phys. Rev. D 70, 074505 (2004). [Erratum: Phys. Rev. D 72, 059903(2005)].

[59] Kaczmarek, O. and Zantow, F. Static quark-antiquark interactions in zero and finite temperature qcd: I. heavy quark free energies, running coupling, and quarkonium binding. Phys. Rev. D 71, 114510 (2005).

[60] Borsanyi, S. et al. QCD equation of state at nonzero chemical potential: continuum results with physical quark masses at order $\mu^{2}$. JHEP 08, 053 (2012).

[61] Bellac, M. L. Thermal Field Theory. Cambridge Monographs on Mathematical Physics (Cambridge University Press, 2011).

[62] Linnyk, O., Bratkovskaya, E. and Cassing, W. Effective QCD and transport description of dilepton and photon production in heavy-ion collisions and elementary processes. Prog. Part. Nucl. Phys. 87, 50-115 (2016). 
[63] Pisarski, R. D. Scattering Amplitudes in Hot Gauge Theories. Phys. Rev. Lett. 63, 1129 (1989).

[64] Lebedev, V. V. and Smilga, A. V. Spectrum of Quark - Gluon Plasma. Annals Phys. 202, 229-270 (1990).

[65] Blaizot, J.-P. and Iancu, E. The Quark gluon plasma: Collective dynamics and hard thermal loops. Phys. Rept. 359, 355-528 (2002).

[66] Peshier, A., Kampfer, B., Pavlenko, O. P. and Soff, G. A Massive quasiparticle model of the SU(3) gluon plasma. Phys. Rev. D 54, 2399-2402 (1996).

[67] Pisarski, R. D. Renormalized Gauge Propagator in Hot Gauge Theories. Physica A 158, 146-157 (1989).

[68] Vanderheyden, B. and Baym, G. Selfconsistent approximations in relativistic plasmas: Quasiparticle analysis of the thermodynamic properties. J. Stat. Phys. 93, 843 (1998).

[69] Blaizot, J. P., Iancu, E. and Rebhan, A. Approximately selfconsistent resummations for the thermodynamics of the quark gluon plasma. 1. Entropy and density. Phys. Rev. D 63, 065003 (2001).

[70] Borsanyi, S. et al. The QCD equation of state with dynamical quarks. JHEP 11, 077 (2010).

[71] Bazavov, A. et al. The chiral and deconfinement aspects of the QCD transition. Phys. Rev. D 85, 054503 (2012).

[72] Bazavov, A. et al. Equation of state in ( 2+1 )-flavor QCD. Phys. Rev. D 90, 094503 (2014).

[73] Romatschke, P. Relativistic (Lattice) Boltzmann Equation with Non-Ideal Equation of State. Phys. Rev. D 85, 065012 (2012).

[74] Chakraborty, P. and Kapusta, J. Quasi-Particle Theory of Shear and Bulk Viscosities of Hadronic Matter. Phys. Rev. C 83, 014906 (2011).

[75] Alqahtani, M., Nopoush, M. and Strickland, M. Quasiparticle equation of state for anisotropic hydrodynamics. Phys. Rev. C 92, 054910 (2015).

[76] Albright, M. and Kapusta, J. Quasiparticle Theory of Transport Coefficients for Hadronic Matter at Finite Temperature and Baryon Density. Phys. Rev. C 93, 014903 (2016).

[77] Hosoya, A. and Kajantie, K. Transport Coefficients of QCD Matter. Nucl. Phys. B 250, 666-688 (1985). 
[78] Berrehrah, H., Gossiaux, P.-B., Aichelin, J., Cassing, W. and Bratkovskaya, E. Dynamical collisional energy loss and transport properties of on- and off-shell heavy quarks in vacuum and in the Quark Gluon Plasma. Phys. Rev. C 90, 064906 (2014).

[79] J.D. Cox, D. W. and Medvedev, V. CODATA Key Values for Thermodynamics (Hemisphere Publishing Corp., New York, http://www.codata.org, 1989).

[80] Brazhkin, V. V., Fomin, Y. D., Lyapin, A. G., Ryzhov, V. N. and Trachenko, K. Two liquid states of matter: A dynamic line on a phase diagram. Phys. Rev. E 85, 031203 (2012).

[81] Marty, R., Bratkovskaya, E., Cassing, W., Aichelin, J. and Berrehrah, H. Transport coefficients from the Nambu-Jona-Lasinio model for $S U(3)_{f}$. Phys. Rev. C 88, 045204 (2013).

[82] Kubo, R. Statistical mechanical theory of irreversible processes. 1. General theory and simple applications in magnetic and conduction problems. J. Phys. Soc. Jap. 12, 570-586 (1957).

[83] Aarts, G. and Martinez Resco, J. M. Transport coefficients, spectral functions and the lattice. JHEP 04, 053 (2002).

[84] Ozvenchuk, V., Linnyk, O., Gorenstein, M. I., Bratkovskaya, E. L. and Cassing, W. Shear and bulk viscosities of strongly interacting "infinite" parton-hadron matter within the parton-hadron-string dynamics transport approach. Phys. Rev. C 87, 064903 (2013).

[85] Astrakhantsev, N., Braguta, V. and Kotov, A. Temperature dependence of shear viscosity of $S U(3)$-gluodynamics within lattice simulation. JHEP 04, 101 (2017).

[86] Bernhard, J. E., Moreland, J. S. and Bass, S. A. Bayesian estimation of the specific shear and bulk viscosity of quark-gluon plasma. Nature Phys. 15, 1113-1117 (2019).

[87] Astrakhantsev, N., Braguta, V. and Kotov, A. Temperature dependence of the bulk viscosity within lattice simulation of $S U(3)$ gluodynamics. Phys. Rev. D 98, 054515 (2018).

[88] Meyer, H. B. A Calculation of the bulk viscosity in SU(3) gluodynamics. Phys. Rev. Lett. 100, 162001 (2008).

[89] Bernhard, J. E., Moreland, J. S., Bass, S. A., Liu, J. and Heinz, U. Applying Bayesian parameter estimation to relativistic heavy-ion collisions: simultaneous characterization of the initial state and quark-gluon plasma medium. Phys. Rev. C 94, 024907 (2016).

[90] Yin, Y. Electrical conductivity of the quark-gluon plasma and soft photon spectrum in heavy-ion collisions. Phys. Rev. C 90, 044903 (2014). 
[91] Turbide, S., Rapp, R. and Gale, C. Hadronic production of thermal photons. Phys. Rev. C 69, 014903 (2004).

[92] Akamatsu, Y., Hamagaki, H., Hatsuda, T. and Hirano, T. Can transport peak explain the low-mass enhancement of dileptons at RHIC? J. Phys. G 38, 124184 (2011).

[93] Linnyk, O., Konchakovski, V., Cassing, W. and Bratkovskaya, E. Photon elliptic flow in relativistic heavy-ion collisions: hadronic versus partonic sources. Phys. Rev. C 88, 034904 (2013).

[94] Brandt, B. B., Francis, A., Meyer, H. B. and Wittig, H. Thermal Correlators in the $\rho$ channel of two-flavor QCD. JHEP 03, 100 (2013).

[95] Brandt, B. B., Francis, A., Jäger, B. and Meyer, H. B. Charge transport and vector meson dissociation across the thermal phase transition in lattice QCD with two light quark flavors. Phys. Rev. D 93, 054510 (2016).

[96] Aarts, G. et al. Electrical conductivity and charge diffusion in thermal QCD from the lattice. JHEP 02, 186 (2015).

[97] Cassing, W., Linnyk, O., Steinert, T. and Ozvenchuk, V. Electrical Conductivity of Hot QCD Matter. Phys. Rev. Lett. 110, 182301 (2013).

[98] Puglisi, A., Plumari, S. and Greco, V. Shear viscosity $\eta$ to electric conductivity $\sigma_{e l}$ ratio for the quark-gluon plasma. Phys. Lett. B 751, 326-330 (2015).

[99] Kovtun, P., Son, D. T. and Starinets, A. O. Viscosity in strongly interacting quantum field theories from black hole physics. Phys. Rev. Lett. 94, 111601 (2005).

[100] Jaiswal, A., Friman, B. and Redlich, K. Relativistic second-order dissipative hydrodynamics at finite chemical potential. Phys. Lett. B 751, 548-552 (2015).

[101] Son, D. T. and Starinets, A. O. Hydrodynamics of r-charged black holes. JHEP 03, $052(2006)$.

[102] Son, D. T. and Stephanov, M. A. Dynamic universality class of the QCD critical point. Phys. Rev. D 70, 056001 (2004).

[103] Denicol, G. S., Molnár, E., Niemi, H. and Rischke, D. H. Resistive dissipative magnetohydrodynamics from the Boltzmann-Vlasov equation. Phys. Rev. D 99, 056017 (2019).

[104] Fotakis, J. A., Greif, M., Greiner, C., Denicol, G. S. and Niemi, H. Diffusion processes involving multiple conserved charges: A study from kinetic theory and implications to the fluid-dynamical modeling of heavy ion collisions. Phys. Rev. D 101, 076007 (2020).

[105] Rose, J.-B. et al. Cross-conductivity: novel transport coefficients to constrain the hadronic degrees of freedom of nuclear matter. Phys. Rev. D 101, 114028 (2020). 
[106] Greif, M., Greiner, C. and Denicol, G. S. Electric conductivity of a hot hadron gas from a kinetic approach. Phys. Rev. D 93, 096012 (2016). [Erratum: Phys. Rev. D 96, $059902(2017)]$.

[107] Landau, L. D. and Lifschitz, E. M. Course of theoretical physics. vol. 6: Fluid mechanics (London, 1959).

[108] Denicol, G. S., Niemi, H., Molnár, E. and Rischke, D. H. Derivation of transient relativistic fluid dynamics from the Boltzmann equation. Phys. Rev. D 85, 114047 (2012).

[109] Ding, H.-T., Kaczmarek, O. and Meyer, F. Thermal dilepton rates and electrical conductivity of the QGP from the lattice. Phys. Rev. D 94, 034504 (2016).

[110] Aarts, G., Allton, C., Foley, J., Hands, S. and Kim, S. Spectral functions at small energies and the electrical conductivity in hot quenched lattice qcd. Phys. Rev. Lett. 99, 022002 (2007).

[111] Astrakhantsev, N. et al. Lattice study of the electromagnetic conductivity of the quark-gluon plasma in an external magnetic field. Phys. Rev. D 102, 054516 (2020).

[112] Greif, M., Bouras, I., Greiner, C. and Xu, Z. Electric conductivity of the quark-gluon plasma investigated using a perturbative QCD based parton cascade. Phys. Rev. D 90, 094014 (2014).

[113] Finazzo, S. I. and Noronha, J. Holographic calculation of the electric conductivity of the strongly coupled quark-gluon plasma near the deconfinement transition. Phys. Rev. D 89, 106008 (2014).

[114] Hammelmann, J., Torres-Rincon, J. M., Rose, J.-B., Greif, M. and Elfner, H. Electrical conductivity and relaxation via colored noise in a hadronic gas. Phys. Rev. D 99, 076015 (2019).

[115] Torres-Rincon, J. M. Hadronic Transport Coefficients from Effective Field Theories. Ph.D. thesis, Universidad Complutense de Madrid (2012).

[116] Amato, A. et al. Electrical conductivity of the quark-gluon plasma across the deconfinement transition. Phys. Rev. Lett. 111, 172001 (2013).

[117] Rougemont, R., Noronha, J. and Noronha-Hostler, J. Suppression of baryon diffusion and transport in a baryon rich strongly coupled quark-gluon plasma. Phys. Rev. Lett. 115, 202301 (2015).

[118] Song, H. and Heinz, U. W. Multiplicity scaling in ideal and viscous hydrodynamics. Phys. Rev. C 78, 024902 (2008).

[119] Shen, C. et al. The iEBE-VISHNU code package for relativistic heavy-ion collisions. Comput. Phys. Commun. 199, 61-85 (2016). 
[120] Denicol, G. S. et al. Net baryon diffusion in fluid dynamic simulations of relativistic heavy-ion collisions. Phys. Rev. C 98, 034916 (2018).

[121] Cassing, W. and Bratkovskaya, E. Parton transport and hadronization from the dynamical quasiparticle point of view. Phys. Rev. C 78, 034919 (2008).

[122] Cassing, W. From Kadanoff-Baym dynamics to off-shell parton transport. Eur. Phys. J. ST 168, 3-87 (2009).

[123] Bratkovskaya, E., Cassing, W., Konchakovski, V. and Linnyk, O. Parton-HadronString Dynamics at Relativistic Collider Energies. Nucl. Phys. A 856, 162-182 (2011).

[124] Soloveva, E. et al. Exploring the partonic phase at finite chemical potential within a covariant off-shell transport approach. J. Phys. Conf. Ser. 1667, 012040 (2020).

[125] Schwinger, J. S. Brownian motion of a quantum oscillator. J. Math. Phys. 2, 407-432 (1961).

[126] Keldysh, L. V. Diagram technique for nonequilibrium processes. Zh. Eksp. Teor. Fiz. 47, 1515-1527 (1964).

[127] Cornwall, J. M., Jackiw, R. and Tomboulis, E. Effective Action for Composite Operators. Phys. Rev. D 10, 2428-2445 (1974).

[128] Danielewicz, P. Quantum Theory of Nonequilibrium Processes. 1. Annals Phys. 152, 239-304 (1984).

[129] Ivanov, Y. B., Knoll, J. and Voskresensky, D. N. Selfconsistent approximations to nonequilibrium many body theory. Nucl. Phys. A 657, 413-445 (1999).

[130] Cassing, W. and Juchem, S. Semiclassical transport of particles with dynamical spectral functions. Nucl. Phys. A 665, 377-400 (2000).

[131] Kadanoff, L. and Baym, G. Quantum Statistical Mechanics. Benjamin, New York 203 pp (1962).

[132] Botermans, W. and Malfliet, R. Quantum transport theory of nuclear matter. Phys. Rept. 198, 115-194 (1990).

[133] Cassing, W. and Juchem, S. Semiclassical transport of hadrons with dynamical spectral functions in A + A collisions at SIS / AGS energies. Nucl. Phys. A 672, 417-445 (2000).

[134] Bratkovskaya, E. L. and Cassing, W. Dilepton production and off-shell transport dynamics at SIS energies. Nucl. Phys. A 807, 214-250 (2008).

[135] Cassing, W. Transport Theories for Strongly-Interacting Systems: Applications to Heavy-Ion Collisions. Lect. Notes Phys. 989, pp. (2021). 
[136] Wong, C.-Y. Dynamics of nuclear fluid. VIII. Time-dependent Hartree-Fock approximation from a classical point of view. Phys. Rev. C 25, 1460-1475 (1982).

[137] Juchem, S. Nonequilibrium Quantum-Field Dynamics and Off-Shell Transport. Ph.D. thesis, Giessen U. (2003).

[138] Ozvenchuk, V., Linnyk, O., Gorenstein, M., Bratkovskaya, E. and Cassing, W. Dynamical equilibration of strongly interacting "infinite" parton matter within the parton-hadron-string dynamics transport approach. Phys. Rev. C 87, 024901 (2013).

[139] Bengtsson, H.-U. and Sjostrand, T. The Lund Monte Carlo for Hadronic Processes: Pythia Version 4.8. Comput. Phys. Commun. 46, 43 (1987).

[140] Lin, Z.-W., Ko, C. M., Li, B.-A., Zhang, B. and Pal, S. A Multi-phase transport model for relativistic heavy ion collisions. Phys. Rev. C 72, 064901 (2005).

[141] Moreau, P., Linnyk, O., Cassing, W. and Bratkovskaya, E. Examinations of the early degrees of freedom in ultrarelativistic nucleus-nucleus collisions. Phys. Rev. C 93, 044916 (2016).

[142] Konchakovski, V., Bratkovskaya, E., Cassing, W., Toneev, V. and Voronyuk, V. Rise of azimuthal anisotropies as a signature of the Quark-Gluon-Plasma in relativistic heavy-ion collisions. Phys. Rev. C 85, 011902 (2012).

[143] Moreau, P. Dynamical description of relativistic heavy-ion collisions out-of equilibrium. Ph.D. thesis, Goethe U., Frankfurt (main) (2019).

[144] Ryblewski, R. and Florkowski, W. Highly-anisotropic hydrodynamics in $3+1$ spacetime dimensions. Phys. Rev. C 85, 064901 (2012).

[145] Cleymans, J., Oeschler, H., Redlich, K. and Wheaton, S. Comparison of chemical freeze-out criteria in heavy-ion collisions. Phys. Rev. C 73, 034905 (2006).

[146] Akiba, Y. et al. Particle production in $\mathrm{Au}+\mathrm{Au}$ collisions from BNL E866. Nucl. Phys. A 610, 139C-152C (1996).

[147] Lacasse, R. et al. Hadron yields and spectra in Au + Au collisions at the AGS. Nucl. Phys. A 610, 153C-164C (1996).

[148] Ahmad, S. et al. Lambda production by 11.6-A/GeV/c Au beam on Au target. Phys. Lett. B 382, 35-39 (1996). [Erratum: Phys. Lett. B 386, 496-496 (1996)].

[149] Barrette, J. et al. Lambda production and flow in $\mathrm{Au}+\mathrm{Au}$ collisions at 11.5-AGeV/c. Phys. Rev. C 63, 014902 (2001).

[150] Albergo, S. et al. Lambda spectra in 11.6-A-GeV/c Au Au collisions. Phys. Rev. Lett. 88, 062301 (2002). 
[151] Alt, C. et al. Energy and centrality dependence of anti-p and p production and the anti-Lambda/anti-p ratio in $\mathrm{Pb}+\mathrm{Pb}$ collisions between 20/A-GeV and 158/A-Gev. Phys. Rev. C 73, 044910 (2006).

[152] Alt, C. et al. Pion and kaon production in central $\mathrm{Pb}+\mathrm{Pb}$ collisions at 20-A and 30-A-GeV: Evidence for the onset of deconfinement. Phys. Rev. C 77, 024903 (2008).

[153] Alt, C. et al. Energy dependence of Lambda and Xi production in central $\mathrm{Pb}+\mathrm{Pb}$ collisions at A-20, A-30, A-40, A-80, and A-158 GeV measured at the CERN Super Proton Synchrotron. Phys. Rev. C 78, 034918 (2008).

[154] Afanasiev, S. V. et al. Energy dependence of pion and kaon production in central $\mathrm{Pb}+\mathrm{Pb}$ collisions. Phys. Rev. C66, 054902 (2002).

[155] Afanasiev, S. V. et al. Energy dependence of pion and kaon production in central $\mathrm{Pb}+\mathrm{Pb}$ collisions. Phys. Rev. C 66, 054902 (2002).

[156] Anticic, T. et al. Centrality dependence of proton and antiproton spectra in $\mathrm{Pb}+\mathrm{Pb}$ collisions at $40 \mathrm{~A} \mathrm{GeV}$ and $158 \mathrm{~A} \mathrm{GeV}$ measured at the CERN SPS. Phys. Rev. C 83, 014901 (2011).

[157] Anticic, T. et al. System-size and centrality dependence of charged kaon and pion production in nucleus-nucleus collisions at $40 \mathrm{~A} \mathrm{GeV}$ and158 A GeV beam energy. Phys. Rev. C 86, 054903 (2012).

[158] Ahle, L. et al. Excitation function of $\mathrm{K}+$ and $\mathrm{pi}+$ production in $\mathrm{Au}+\mathrm{Au}$ reactions at 2/A-GeV to $10 / \mathrm{A}-\mathrm{GeV}$. Phys. Lett. B 476, 1-8 (2000).

[159] Ahle, L. et al. An Excitation function of $\mathrm{K}$ - and $\mathrm{K}+$ production in $\mathrm{Au}+\mathrm{Au}$ reactions at the AGS. Phys. Lett. B 490, 53-60 (2000).

[160] Anticic, T. et al. Lambda and anti-Lambda production in central $\mathrm{Pb}-\mathrm{Pb}$ collisions at 40-A-GeV, 80-A-GeV and 158-A-GeV. Phys. Rev. Lett. 93, 022302 (2004).

[161] Adamczyk, L. et al. Bulk Properties of the Medium Produced in Relativistic HeavyIon Collisions from the Beam Energy Scan Program. Phys. Rev. C 96, 044904 (2017).

[162] Adler, S. S. et al. Identified charged particle spectra and yields in $\mathrm{Au}+\mathrm{Au}$ collisions at $\sqrt{s_{N N}}=200 \mathrm{GeV}$. Phys. Rev. C 69, 034909 (2004).

[163] Agakishiev, G. et al. Strangeness Enhancement in $\mathrm{Cu}+\mathrm{Cu}$ and $\mathrm{Au}+\mathrm{Au}$ Collisions at $\sqrt{s_{N N}}=200 \mathrm{GeV}$. Phys. Rev. Lett. 108, 072301 (2012).

[164] Bearden, I. G. et al. Charged meson rapidity distributions in central $\mathrm{Au}+\mathrm{Au}$ collisions at $\mathrm{s}(\mathrm{NN})^{* *}(1 / 2)=200-\mathrm{GeV}$. Phys. Rev. Lett. 94, 162301 (2005).

[165] Arsene, I. et al. Centrality dependent particle production at $\mathrm{y}=0$ and $\mathrm{y} \sim 1$ in $\mathrm{Au}+$ Au collisions at $\mathrm{s}(\mathrm{NN})^{* *}(1 / 2)=200-\mathrm{GeV}$. Phys. Rev. C 72, 014908 (2005). 
[166] Ollitrault, J.-Y. Anisotropy as a signature of transverse collective flow. Phys. Rev. D 46, 229-245 (1992).

[167] Sorge, H. Highly sensitive centrality dependence of elliptic flow: A novel signature of the phase transition in QCD. Phys. Rev. Lett. 82, 2048-2051 (1999).

[168] Voloshin, S. A., Poskanzer, A. M. and Snellings, R. Collective phenomena in noncentral nuclear collisions. Landolt-Bornstein 23, 293-333 (2010).

[169] Adamczyk, L. et al. Beam-Energy Dependence of Directed Flow of $\Lambda, \bar{\Lambda}, K^{ \pm}, K_{s}^{0}$ and $\phi$ in $\mathrm{Au}+\mathrm{Au}$ Collisions. Phys. Rev. Lett. 120, 062301 (2018).

[170] Romatschke, P. and Romatschke, U. Viscosity Information from Relativistic Nuclear Collisions: How Perfect is the Fluid Observed at RHIC? Phys. Rev. Lett. 99, 172301 (2007).

[171] Song, H., Bass, S. A., Heinz, U., Hirano, T. and Shen, C. $200 \mathrm{~A} \mathrm{GeV} \mathrm{Au+Au}$ collisions serve a nearly perfect quark-gluon liquid. Phys. Rev. Lett. 106, 192301 (2011). [Erratum: Phys.Rev.Lett. 109, 139904 (2012)].

[172] Adams, J. et al. Azimuthal anisotropy in $\mathrm{Au}+\mathrm{Au}$ collisions at $\mathrm{s}(\mathrm{NN})^{* *}(1 / 2)=200-$ GeV. Phys. Rev. C 72, 014904 (2005).

[173] Back, B. B. et al. Centrality and pseudorapidity dependence of elliptic flow for charged hadrons in $\mathrm{Au}+\mathrm{Au}$ collisions at $\mathrm{s}(\mathrm{NN})^{* *}(1 / 2)=200-\mathrm{GeV}$. Phys. Rev. C 72, 051901 (2005).

[174] Adamczyk, L. et al. Centrality dependence of identified particle elliptic flow in relativistic heavy ion collisions at $\sqrt{s_{N N}}=7.7-62.4 \mathrm{GeV}$. Phys. Rev. C 93, 014907 (2016).

[175] Fuseau, D., Steinert, T. and Aichelin, J. Phase diagram of the Polyakov-Nambu-Jona-Lasinio approach for finite chemical potentials. Phys. Rev. C 101, 065203 (2020).

[176] Nambu, Y. and Jona-Lasinio, G. Dynamical model of elementary particles based on an analogy with superconductivity. i. Phys. Rev. 122, 345-358 (1961).

[177] Bardeen, J., Cooper, L. N. and Schrieffer, J. R. Microscopic theory of superconductivity. Physical Review 106, 162-164 (1957).

[178] Bogolyubov, N. N. A New method in the theory of superconductivity. I. Sov. Phys. JETP 7, 41-46 (1958).

[179] Klevansky, S. P. The Nambu-Jona-Lasinio model of quantum chromodynamics. Rev. Mod. Phys. 64, 649-708 (1992).

[180] Hatsuda, T. and Kunihiro, T. QCD phenomenology based on a chiral effective Lagrangian. Phys. Rept. 247, 221-367 (1994). 
[181] Fukushima, K. Chiral effective model with the Polyakov loop. Phys. Lett. B 591, $277-284$ (2004).

[182] Megias, E., Ruiz Arriola, E. and Salcedo, L. L. Polyakov loop in chiral quark models at finite temperature. Phys. Rev. D 74, 065005 (2006).

[183] Ratti, C., Thaler, M. A. and Weise, W. Phases of QCD: Lattice thermodynamics and a field theoretical model. Phys. Rev. D 73, 014019 (2006).

[184] Hansen, H. et al. Mesonic correlation functions at finite temperature and density in the Nambu-Jona-Lasinio model with a Polyakov loop. Phys. Rev. D 75, 065004 (2007).

[185] Torres-Rincon, J. M., Sintes, B. and Aichelin, J. Flavor dependence of baryon melting temperature in effective models of QCD. Phys. Rev. C 91, 065206 (2015).

[186] Rehberg, P., Klevansky, S. P. and Hufner, J. Elastic scattering and transport coefficients for a quark plasma in $\operatorname{SU}-\mathrm{f}(3)$ at finite temperatures. Nucl. Phys. A 608, 356-388 (1996).

[187] Rehberg, P. and Klevansky, S. P. One loop integrals at finite temperature and density. Annals Phys. 252, 422-457 (1996).

[188] Rehberg, P., Klevansky, S. P. and Hufner, J. Hadronization in the SU(3) NambuJona-Lasinio model. Phys. Rev. C 53, 410-429 (1996).

[189] Torres-Rincon, J. M. and Aichelin, J. Equation of state of a quark-meson mixture in the improved Polyakov-Nambu-Jona-Lasinio model at finite chemical potential. Phys. Rev. C 96, 045205 (2017).

[190] Kurkela, A. and Vuorinen, A. Cool quark matter. Phys. Rev. Lett. 117, 042501 (2016).

[191] Costa, P., Hansen, H., Ruivo, M. C. and de Sousa, C. A. How parameters and regularization affect the PNJL model phase diagram and thermodynamic quantities. Phys. Rev. D 81, 016007 (2010).

[192] Borsanyi, S. et al. QCD Crossover at Finite Chemical Potential from Lattice Simulations. Phys. Rev. Lett. 125, 052001 (2020).

[193] Blanquier, E. Le modèle de Polyakov, Nambu et Jona-Lasinio et ses applications pour décrire les particules sub-nucléaires. Ph.D. thesis, Toulouse III U. (2013).

[194] Deb, P., Kadam, G. P. and Mishra, H. Estimating transport coefficients in hot and dense quark matter. Phys. Rev. D 94, 094002 (2016).

[195] Zhuang, P., Hufner, J., Klevansky, S. P. and Neise, L. Transport properties of a quark plasma and critical scattering at the chiral phase transition. Phys. Rev. D 51, 3728-3738 (1995). 
[196] Sasaki, C. and Redlich, K. Transport coefficients near chiral phase transition. Nucl. Phys. A 832, 62-75 (2010).

[197] Demir, N. and Bass, S. A. Shear-Viscosity to Entropy-Density Ratio of a Relativistic Hadron Gas. Phys. Rev. Lett. 102, 172302 (2009).

[198] Rose, J. B., Torres-Rincon, J. M., Schäfer, A., Oliinychenko, D. R. and Petersen, $\mathrm{H}$. Shear viscosity of a hadron gas and influence of resonance lifetimes on relaxation time. Phys. Rev. C 97, 055204 (2018).

[199] Heffernan, M., Jeon, S. and Gale, C. Hadronic transport coefficients from the linear $\sigma$ model at finite temperature. Phys. Rev. C 102, 034906 (2020).

[200] Nakamura, A. and Sakai, S. Transport coefficients of gluon plasma. Phys. Rev. Lett. 94, 072305 (2005).

[201] Sakai, S. and Nakamura, A. Lattice calculation of the QGP viscosities: Present results and next project. PoS LATTICE2007, 221 (2007).

[202] Caron-Huot, S., Kovtun, P., Moore, G. D., Starinets, A. and Yaffe, L. G. Photon and dilepton production in supersymmetric Yang-Mills plasma. JHEP 12, 015 (2006).

[203] Goldenfeld, N. Lectures on phase transitions and the renormalization group. Frontiers in Physics Vol. 85 (Westview, Boulder, CO) (1992).

[204] Costa, P., de Sousa, C. A., Ruivo, M. C. and Kalinovsky, Y. L. The QCD critical end point in the SU(3) Nambu-Jona-Lasinio model. Phys. Lett. B 647, 431-435 (2007).

[205] Costa, P., de Sousa, C. A., Ruivo, M. C. and Hansen, H. The QCD Critical End Point in the Context of the Polyakov-Nambu-Jona-Lasinio Model. Acta Phys. Polon. Supp. 5, 523-528 (2012).

[206] Schaefer, B. J. and Wagner, M. QCD critical region and higher moments for three flavor models. Phys. Rev. D 85, 034027 (2012).

[207] Fujii, H. and Ohtani, M. Sigma and hydrodynamic modes along the critical line. Phys. Rev. D 70, 014016 (2004).

[208] Hohenberg, P. C. and Halperin, B. I. Theory of Dynamic Critical Phenomena. Rev. Mod. Phys. 49, 435-479 (1977).

[209] Kadanoff, L. P. and Swift, J. Transport Coefficients near the Liquid-Gas Critical Point. Phys. Rev. 166, 89-101 (1968).

[210] Stephanov, M. and Yin, Y. Hydrodynamics with parametric slowing down and fluctuations near the critical point. Phys. Rev. D 98, 036006 (2018).

[211] Plumari, S., Alberico, W. M., Greco, V. and Ratti, C. Recent thermodynamic results from lattice QCD analyzed within a quasi-particle model. Phys. Rev. D 84, 094004 (2011). 
[212] Scardina, F., Das, S. K., Minissale, V., Plumari, S. and Greco, V. Estimating the charm quark diffusion coefficient and thermalization time from D meson spectra at energies available at the BNL Relativistic Heavy Ion Collider and the CERN Large Hadron Collider. Phys. Rev. C 96, 044905 (2017).

[213] Ehrenfest, P. Phasenumwandlungen im ueblichen und erweiterten Sinn, classifiziert nach dem entsprechenden Singularitaeten des thermodynamischen Potentiales. Verhandlingen der Koninklijke Akademie van Wetenschappen (Amsterdam) 36: 153-157; Communications from the Physical Laboratory of the University of Leiden, Supplement No. $75 b$ (1933).

[214] Cea, P., Cosmai, L. and Papa, A. Critical line of 2+1 flavor QCD: Toward the continuum limit. Phys. Rev. D 93, 014507 (2016).

[215] Borsanyi, S. et al. Is there still any $T_{c}$ mystery in lattice QCD? Results with physical masses in the continuum limit III. JHEP 09, 073 (2010).

[216] Wölfle, P., Schmalian, J. and Abrahams, E. Strong coupling theory of heavy fermion criticality II. Rept. Prog. Phys. 80, 044501 (2017).

[217] Parotto, P. et al. QCD equation of state matched to lattice data and exhibiting a critical point singularity. Phys. Rev. C 101, 034901 (2020).

[218] Weber, J. H., Bazavov, A. and Petreczky, P. Equation of state in $(2+1)$ flavor QCD at high temperatures. PoS Confinement2018, 166 (2019).

[219] Hatta, Y. and Ikeda, T. Universality, the QCD critical / tricritical point and the quark number susceptibility. Phys. Rev. D 67, 014028 (2003).

[220] Pisarski, R. D. and Wilczek, F. Remarks on the Chiral Phase Transition in Chromodynamics. Phys. Rev. D 29, 338-341 (1984).

[221] Berges, J. and Rajagopal, K. Color superconductivity and chiral symmetry restoration at nonzero baryon density and temperature. Nucl. Phys. B 538, 215-232 (1999).

[222] de Forcrand, P. and Philipsen, O. The QCD phase diagram for three degenerate flavors and small baryon density. Nucl. Phys. B 673, 170-186 (2003).

[223] Pelissetto, A. and Vicari, E. Critical phenomena and renormalization-group theory. Physics Reports 368, 549-727 (2002).

[224] Sasaki, C., Friman, B. and Redlich, K. Chiral phase transition in the presence of spinodal decomposition. Phys. Rev. D 77, 034024 (2008).

[225] Lu, Y., Du, Y.-L., Cui, Z.-F. and Zong, H.-S. Critical behaviors near the (tri-)critical end point of QCD within the NJL model. Eur. Phys. J. C 75, 495 (2015).

[226] Du, Y.-L. et al. Susceptibilities and critical exponents within the Nambu Jona-Lasinio model. Int. J. Mod. Phys. A 30, 1550199 (2015). 
[227] Dore, T., Noronha-Hostler, J. and McLaughlin, E. Far-from-equilibrium search for the QCD critical point. Phys. Rev. D 102, 074017 (2020).

[228] Du, L., An, X. and Heinz, U. Baryon transport and the QCD critical point. arXiv:2107.02302 (2021).

[229] Nonaka, C. and Asakawa, M. Hydrodynamical evolution near the QCD critical end point. Phys. Rev. C 71, 044904 (2005).

[230] Motta, M., Stiele, R., Alberico, W. M. and Beraudo, A. Isentropic evolution of the matter in heavy-ion collisions and the search for the critical endpoint. Eur. Phys. J. C 80, 770 (2020).

[231] Karthein, J. M. et al. Strangeness-neutral equation of state for QCD with a critical point. Eur. Phys. J. Plus 136, 621 (2021).

[232] Günther, J. et al. The QCD equation of state at finite density from analytical continuation. EPJ Web Conf. 137, 07008 (2017).

[233] Berrehrah, H., Bratkovskaya, E., Cassing, W., Gossiaux, P. B. and Aichelin, J. Heavy quark scattering and quenching in a QCD medium at finite temperature and chemical potential. Phys. Rev. C 91, 054902 (2015).

[234] Karsch, F., Kharzeev, D. and Tuchin, K. Universal properties of bulk viscosity near the QCD phase transition. Phys. Lett. B 663, 217-221 (2008).

[235] Moore, G. D. and Saremi, O. Bulk viscosity and spectral functions in QCD. JHEP 09, 015 (2008).

[236] Onuki, A. Dynamic equations and bulk viscosity near the gas-liquid critical point. Phys. Rev. E 55, 403-420 (1997).

[237] Monnai, A., Mukherjee, S. and Yin, Y. Phenomenological Consequences of Enhanced Bulk Viscosity Near the QCD Critical Point. Phys. Rev. C 95, 034902 (2017).

[238] Adam, J. et al. Bulk properties of the system formed in $A u+A u$ collisions at $\sqrt{s_{\mathrm{NN}}}$ $=14.5 \mathrm{GeV}$ at the BNL STAR detector. Phys. Rev. C 101, 024905 (2020).

[239] Adamczyk, L. et al. Elliptic flow of identified hadrons in $\mathrm{Au}+\mathrm{Au}$ collisions at $\sqrt{s_{N N}}=$ 7.7-62.4 GeV. Phys. Rev. C 88, 014902 (2013). 


\section{Acknowledgements}

I would like to thank various people who supported and inspired me through the last three years in one way or another. First of all, I would like to sincerely thank my scientific advisor, Prof. Dr. E. Bratkovskaya for her patient guidance, support, and constructive feedback during my $\mathrm{PhD}$ studies. I am very grateful for the opportunities that she has provided me to broaden my scope of knowledge and to improve as a research scientist in the past three years. I greatly appreciate the collaboration with Prof. Dr. J. Aichelin for his oversight, fascinating discussions, and valuable feedback. Moreover, I am also very grateful to Prof. Dr. J. Aichelin for his hospitality during my visits to Nantes.

I would like to express my gratitude and appreciation to Prof. Dr. Dr. W. Cassing for his helpful discussions and critical reading of my thesis. I also wish to thank the whole PHSD team and collaborators: Dr. T. Song, Dr. G. Coci, I. Grishmanovskii, V. Kireyeu, Dr. V. Voronyuk, Dr. L. Oliva, Dr. Pierre Moreau, and Dr. Juan M. Torres-Rincon. Especially, I am thankful to my best office mate, Dr. L. Oliva, who was part of the PHSD group till 2021, for the great time working and spending leisure time together. I am very thankful to V. Kireyeu, and Dr. V. Voronyuk for the helpful discussions about the experimental techniques and more.

My gratitude also goes to my master scientific advisors Dr. E. Luschevskaya and Prof. O. Teryaev, and many scientists from ITEP and NRNU MEPhI, in particular, Prof. A. Gorsky, Prof. S. Blinnikov, Dr. V. Braguta, Dr. A. Kotov, Prof. B. Martemyanov, Prof. V. Zakharov, Prof. V. Shevchenko, Prof. S. Popruzhenko, Prof. A. Taranenko who inspired me and who drive my curiosity.

I would also like to acknowledge the support from The Helmholtz Graduate School for Hadron and Ion Research "HGS-HIRe for FAIR" and Dr. G. Burau and Prof. Dr. H. Büsching for the great organization of many events as well as leisure activities.

I would like to send my regards to the working group of Prof. Dr. Dr. h.c. M. Bleicher, in particular to P. Hillmann and T. Reichert for many fruitful discussions about physics and more.

I am also very thankful for the opportunity to participate in the CRC-TR 211 'Stronginteraction matter under extreme conditions' as an associate member. I would like to express my gratitude to the working group of Prof. Dr. C. Greiner, and especially to Jan Fotakis, for such fruitful collaboration and valuable feedback within the CRC project and outside it.

Finally, I would like to thank my friends and family who made my life more meaningful and enjoyable. I am very thankful to Dr. M. Lizunova and Dr. N. Gusinskaya for many adventures and great support when it is needed! Last but not least, I would like to express special thanks to my beloved husband, V. Tulsky. His creativity and sense of humor during our discussions greatly encourage and support me. 